\title{
A Model of Neutrino Masses and Mixings and Its Phenomenological Implications
}

\author{
Trinh Thi Ngoc Le \\ Quang Tri, Vietnam \\ B.Sc., Hue University's College of Education, 2012
}

\begin{abstract}
A Thesis presented to the Graduate Faculty
of the the University of Virginia in Candidacy for the Degree of

Doctor of Philosophy

Department of Physics

University of Virginia
\end{abstract}

May, 2017 
Dedicated to my mother Van T. Phan,

father Minh V. Le, sister Tram-Anh T. Le,

and

Vu Tran 


\section{Acknowledgements}

The work presented here would have not been possible without the help and support of many people to whom I owe many thanks.

Firstly, I would like to express my sincere gratitude to my advisor Prof. Pham Quang Hung for the continuous support of my $\mathrm{PhD}$ study and related research, for his patience, motivation, and immense knowledge. With him I was trained to work independently and to handle unexpected situations. His guidance helped me in all the time of research and writing this thesis. I could not have imagined having a better advisor and mentor for my $\mathrm{PhD}$ study.

I would like to thank members of my research review committee: Prof. Hung, Prof. Craig Group, Prof. Michael Fowler, for taking time to review annually my research and giving me their valuable feedback on the progress of my research. Besides my advisor, I am very grateful to the rest of my thesis committee: Prof. Group, Prof. Hank Thacker, Prof. Trinh Xuan Thuan, for giving time out of their schedules to review my thesis, for their insightful comments, suggestions and also for my doctoral defense.

I also thank my colleagues and friends Van Que Tran and Prof. Tzu-Chiang Yuan for their

collaborations in two projects, for their warm welcomes and hospitalities when I was visiting at the Institute of Physics, Academia Sinica, Taiwan.

My deepest gratitude goes to my parents and my little sister who are always in my heart. They are my moral supports to help me overcome obstacles in my life. With them by my side all 
the time I have been motivated to accomplish the goals of my life.

Furthermore, I am also grateful to: The University of Virginia and the Department of Physics for giving me a chance to persuade my $\mathrm{PhD}$; the lab instructor, Dr. Maksim Bychkov for his thoughtful guidance and training on my teaching, for giving me an opportunity to contribute to lab works; all of professors who have taught and supported me in the first two years: Prof. Fowler, Prof. Cox, Prof. Vaman, Prof. Zheng, Prof. Sackett, Prof. Hirosky, Prof. Kolomeisky; Dr. Amy Clobes, the Director of Professional Development at UVA's Office of Graduate and Postdoctoral Affairs, for her guidance and encouragement; Vu Tran for supporting me spiritually throughout writing this thesis and my life in general; all of my friends at UVA, especially Dat Duong, Vinh Hoang, and friends elsewhere for a lot of cheerful moments that I was having with.

Last but not the least, I would like to thank all people whom I could not explicitly mention here but their presences are such valuable memories of my $\mathrm{PhD}$ life. 


\begin{abstract}
We present a model of neutrino masses and mixings within the framework of the EW- $\nu_{R}$ model in which the experimentally desired form of the PMNS matrix is obtained by applying an $A_{4}$ symmetry to the Higgs singlet sector responsible for the neutrino Dirac mass matrix. This mechanism naturally avoids potential conflict with the LHC data which severely constrains the Higgs sector, in particular the Higgs doublets. Moreover, by making a simple ansätz we extract $M_{l} M_{l}^{\dagger}$ for the charged lepton sector. A similar ansätz is proposed for the quark sector in order to construct quark mass matrices. The sources of masses for the neutrinos are entirely different from those for the charged leptons and for the quarks and this might explain why $U_{P M N S}$ is very different from $V_{C K M}$. Two interesting phenomenological implications on $\mu \rightarrow e \gamma$ and $\mu-e$ conversion are investigated within the model. At the limit of zero momentum transfer and large mirror lepton masses, we derive a simple formula to relate the conversion rate with the on-shell radiative decay rate of muon into electron. The Yukawa couplings constrained by current limits and projected sensitivities of these processes are found to be small $\sim \mathcal{O}\left(10^{-5}-10^{-3}\right)$, which give rise to distinct signatures in the search of mirror charged leptons and Majorana right-handed neutrinos at the LHC (or planned colliders).
\end{abstract}




\section{Contents}

\section{Chapter}

\begin{tabular}{lll}
\hline & Introduction & 1
\end{tabular}

2 Standard Model Overview $\quad 5$

2.1 Particle families and their interactions $\ldots \ldots \ldots \ldots \ldots \ldots$

2.2 The SM gauge interactions $\ldots \ldots \ldots \ldots \ldots \ldots \ldots$

2.2 .1 Gauge transformations . . . . . . . . . . . . . . . . 7

$2.2 .2 \quad$ Gauging SM interactions $\ldots \ldots \ldots \ldots \ldots \ldots \ldots$

2.3 Higgs Mechanism and how to generate gauge boson masses . . . . . . . . . . . . 21

$2.3 .1 \quad$ Spontaneous symmetry breaking in $U(1)$ gauge theory $\ldots \ldots \ldots$. . . . . . . . 21

$2.3 .2 \quad$ Higgs mechanism for the $S U(2)_{L}$ gauge theory $\ldots \ldots \ldots \ldots \ldots$

2.4 SM gauge theory via Higgs mechanism $\ldots \ldots \ldots \ldots \ldots \ldots$

$2.4 .1 \quad$ Generating masses for gauge bosons $\ldots \ldots \ldots \ldots \ldots$. . . . . . . . . . 25

2.4 .2 Generating masses for fermions $\ldots \ldots \ldots \ldots \ldots$. . . . . . . . . 29

2.4 .3 CKM matrix for quark sector $\ldots \ldots \ldots \ldots \ldots$

2.5 Standard Model: Limitations and Challenges $\ldots \ldots \ldots$. . . . . . . . . . . . . . 33

\begin{tabular}{lll}
\hline 3 & Neutrinos & 35
\end{tabular}

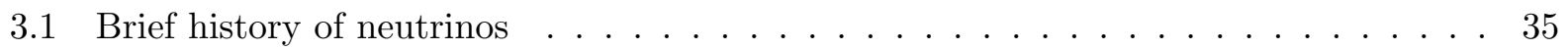

3.2 Neutrino Oscillations . . . . . . . . . . . . . . . . . . . 36

3.3 Neutrino masses and mixings $\ldots \ldots \ldots \ldots \ldots$. . . . . . . . . . . . . . 41 
3.4 Dirac and Majorana Neutrino Masses $\ldots \ldots \ldots$. . . . . . . . . . . . . . 46

3.4 .1 Dirac neutrino mass $\ldots \ldots \ldots \ldots \ldots \ldots$

$3.4 .2 \quad$ Majorana neutrino mass $\ldots \ldots \ldots \ldots \ldots \ldots$

3.5 Seesaw mechanism of neutrino masses $\ldots \ldots \ldots \ldots \ldots$

3.5.1 Type-I Seesaw $\ldots \ldots \ldots \ldots \ldots \ldots \ldots$

3.5 .2 Type-II Seesaw $\ldots \ldots \ldots$. . . . . . . . . . . . . . . . . . . 52

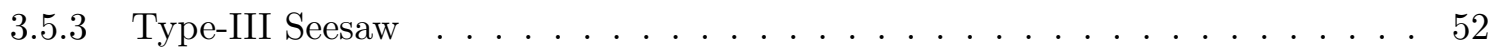

3.6 Left-Right symmetric model . . . . . . . . . . . . . . . . . . . . . . 54

3.7 Overall remarks $\ldots \ldots \ldots \ldots \ldots \ldots$

\begin{tabular}{|lrl}
\hline 4 & The Minimal EW- $\nu_{R}$ Model & 57
\end{tabular}

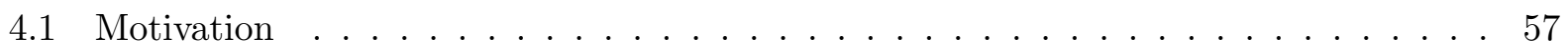

4.2 Definition, gauge group, and particle content $\ldots \ldots \ldots \ldots$. . . . . . . . . 59

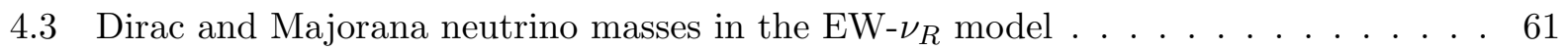

4.4 Charged fermion masses (lepton and quark) . . . . . . . . . . . . . . . . 63

$4.5 \quad \rho$ parameter and possible signatures of EW- $\nu_{R}$ model $\ldots \ldots \ldots \ldots$. . . . . . . . 64

4.6 Phenomenological constraints $\ldots \ldots \ldots \ldots \ldots \ldots$

$4.6 .1 \quad$ Electroweak precision constraints on the EW $\nu_{R}$ model $[71] \ldots$. . . . . . 68

4.6.2 Review of the scalar sector of the EW $\nu_{R}$ model in light of the discovery of the $125-\mathrm{GeV}$ SM-like scalar $[73] \ldots$. . . . . . . . . . . . . . . . 69

\begin{tabular}{|lll}
\hline 5 & Model of Neutrino Masses and Mixings & 73
\end{tabular}

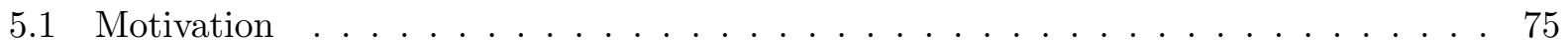

5.2 Assignment of particle content $\ldots \ldots \ldots \ldots \ldots \ldots$

$5.3 \quad$ Neutrino Dirac and Majorana masses with $A_{4}$ symmetry $\ldots \ldots \ldots$. . . . . . . . . 79

5.3 .1 Neutrino Dirac mass matrix . . . . . . . . . . . . . . . . . . . . 79

5.3 .2 Neutrino Majorana mass matrix $\ldots \ldots \ldots \ldots$. . . . . . . . 80

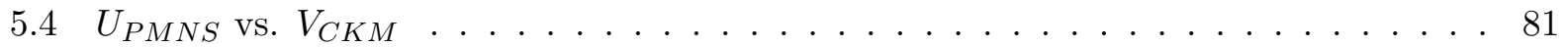


5.4 .1 The search for $U_{l L} \ldots \ldots \ldots \ldots \ldots \ldots \ldots$

$5.4 .2 \quad$ Ansätz for $U_{l L} \ldots \ldots \ldots \ldots \ldots \ldots \ldots \ldots \ldots \ldots$

5.5 Toward charged lepton masses $\ldots \ldots \ldots \ldots$. . . . . . . . . . . 86

5.6 Summary of the chapter $\ldots \ldots \ldots \ldots \ldots$

6 Phenomenological Implications of the Lepton Sector $\quad 90$

$6.1 \quad$ One-loop induced LFV radiative decays $\mu \rightarrow e \gamma \ldots \ldots$. . . . . . . . . . . . . . 91

6.1.1 Review of neutrino and charged lepton masses and mixings in the EW-scale

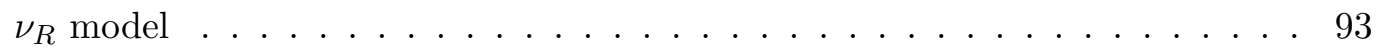

6.1 .2 The calculation $\ldots \ldots \ldots \ldots \ldots \ldots \ldots$

6.1 .3 Numerical Analysis $\ldots \ldots \ldots \ldots \ldots$. . . . . . . . . . . . . . . . 98

6.1 .4 Implications $\ldots \ldots \ldots \ldots \ldots \ldots$

6.1 .5 Summary of the section $\ldots \ldots \ldots \ldots \ldots$

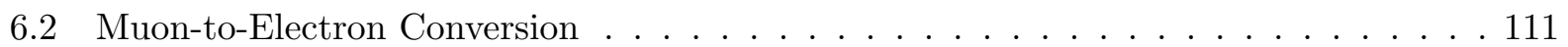

6.2 .1 Mirror Fermion Model Calculation . . . . . . . . . . . . . . . . . . . 113

6.2 .2 The relationship between $\mu-e$ Conversion and $\mu \rightarrow e \gamma \ldots 117$

6.2 .3 Numerical analysis $\ldots \ldots \ldots \ldots$

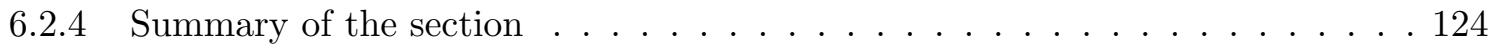

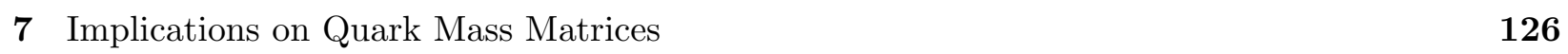

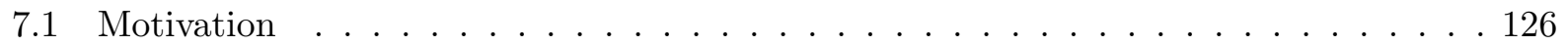

$7.2 \quad$ Relation between $\lambda, \lambda_{u}$ and $\lambda_{d} \ldots \ldots \ldots \ldots \ldots \ldots \ldots \ldots \ldots$

$7.3 \quad$ A general quark mass matrix and its eigenvalues $\ldots \ldots \ldots$. . . . . . . . . . . . 129

7.4 Finding quark mass matrices $\mathcal{M}_{u}$ and $\mathcal{M}_{d} \ldots \ldots \ldots \ldots$. . . . . . . . 131

7.4 .1 Down-quark sector $\ldots \ldots \ldots \ldots \ldots \ldots \ldots$

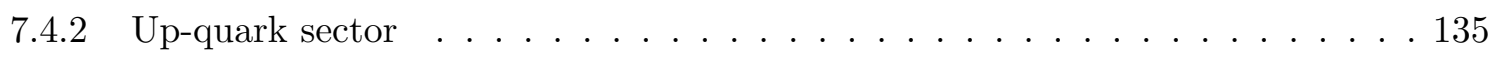

7.5 Agreement with the experimental CKM matrix . . . . . . . . . . . . . . . 140 
8 Conclusion 142

\section{Appendix}

\begin{tabular}{|l|l}
\hline A Effective Lagrangian For $\mu-e$ Conversion & 147
\end{tabular}

\begin{tabular}{lll}
\hline B & Formulas for $\mathcal{I}, \mathcal{J}, \mathcal{I}_{i 0}(i=1, \cdots, 5)$ & $\mathbf{1 5 0}$
\end{tabular}

C Four-Fermion Coupling Constants $C_{V(L, R)}^{(q)} \quad 153$

C.1 $Z$ Boson Exchange $\ldots \ldots \ldots \ldots \ldots$

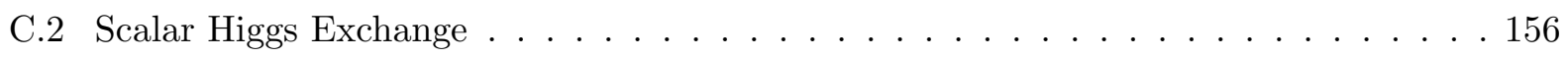

C.3 Box diagram contribution $\ldots \ldots \ldots \ldots$. . . . . . . . . . . . . . . . . . . . . .

\section{Bibliography}




\section{Tables}

\section{Table}

2.1 Gauge quantum numbers of chiral SM fermions . . . . . . . . . . . . . . . . 19

3.1 Sensitivity of different neutrino oscillation experiments . . . . . . . . . . . . . 40

3.2 Mixing parameters from global three-neutrino oscillation data . . . . . . . . . . . 45

$5.1 \quad A_{4}$ assignments for leptons and Higgs fields $\ldots \ldots \ldots \ldots \ldots$

$6.1 \quad$ Matrix elements for $M^{k}(k=0,1,2,3) \ldots \ldots \ldots \ldots \ldots$

6.2 Mixing parameters from global three-neutrino oscillation data taken from [54 [55]. . . 101

7.1 Wolfenstein vs. Wolfenstein-like parameters for CKM matrix . . . . . . . . . . 129

A.1 Values of the dimensionless overlap integrals for aluminum, titanium and gold, evaluated under the assumption that the proton and neutron distributions within each nuclei are the same $[113] . \ldots \ldots \ldots$. . . . . . . . . . . . . . . . . . . . . . . .

A.2 Standard model values of the capture rates for aluminum, titanium and gold in unit of $10^{6} \mathrm{~s}^{-1}$ taken from Ref. $[124] . \ldots \ldots \ldots \ldots$. . . . . . . . . . . . . . . . . . . . . 


\section{Figures}

\section{Figure}

2.1 The Standard Model particle content . . . . . . . . . . . . . . . . . . . . 8

2.2 One-loop process $\nu_{\mu} e \rightarrow \nu_{\mu} e$ results in an infinite cross-section. $\ldots \ldots \ldots$

2.3 Renormalization of weak interactions . . . . . . . . . . . . . . . . . . 15

$2.4 \quad \nu_{e} \bar{\nu}_{e} \rightarrow W^{+} W^{-}$process with a t-channel. $\ldots \ldots \ldots \ldots$

$2.5 \quad \nu_{e} \bar{\nu}_{e} \rightarrow W^{+} W^{-}$with (a) u-channel and (b) s-channel. $\ldots \ldots \ldots \ldots$

2.6 Anomalies from triangle diagrams in a gauge theory $\ldots \ldots \ldots$. . . . . . . . 20

3.1 Solar neutrino observations . . . . . . . . . . . . . . . . . . . . . 37

3.2 Neutrino mass hierarchy $\ldots \ldots \ldots \ldots \ldots \ldots$

$3.3 \quad$ Feynman diagram for neutrinoless double beta decay $\ldots \ldots \ldots$. . . . . . . . . . . . . 49

$3.4 \quad$ Diagrammatic representation for type-I seesaw mechanism . . . . . . . . . . . . . 51

3.5 Diagrammatic representation for type-II seesaw mechanism $\ldots \ldots \ldots$

$3.6 \quad$ Diagrammatic representation for type-III seesaw mechanism . . . . . . . . . . . . 54

4.1 Constrained $\tilde{S}_{S}$ versus $\tilde{S}_{M F} \ldots \ldots \ldots \ldots \ldots \ldots$

4.2 Figure shows the predictions of $\mu\left(\widetilde{H} \rightarrow b \bar{b}, \tau \bar{\tau}, \gamma \gamma, W^{+} W^{-}, Z Z\right)$ in the EW $\nu_{R}$ model for examples 1 and 2 in Dr. Jekyll and example 1, 2 and 3 in Mr. Hyde scenarios as discussed in $\mid 73]$, in comparison with corresponding best fit values by

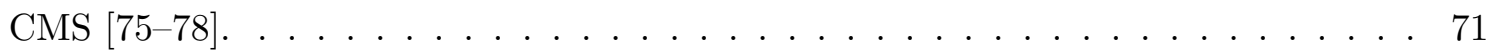

6.1 One-loop induced Feynman diagram for $l_{i} \rightarrow l_{j} \gamma$ in EW-scale $\nu_{R}$ model $\ldots . . .95$ 
6.2 Contour plots of $\log _{10} B(\mu \rightarrow e \gamma)$ and $\log _{10} \Delta a_{\mu}$ with $g_{0 S}=g_{0 S}^{\prime}$ and $g_{1 S}=g_{1 S}^{\prime}=0$.

6.3 Contour plots of $\log _{10} B(\mu \rightarrow e \gamma)$ and $\log _{10} \Delta a_{\mu}$ with $g_{0 S}=g_{0 S}^{\prime}$ and $g_{1 S}=g_{1 S}^{\prime}=$

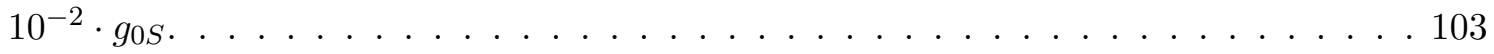

6.4 Contour plots of $\log _{10} B(\mu \rightarrow e \gamma)$ and $\log _{10} \Delta a_{\mu}$ with $g_{0 S}=g_{0 S}^{\prime}$ and $g_{1 S}=g_{1 S}^{\prime}=$

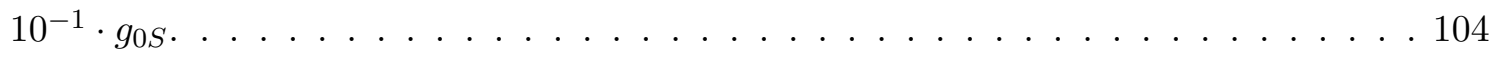

6.5 Contour plots of $\log _{10} B(\mu \rightarrow e \gamma)$ and $\log _{10} \Delta a_{\mu}$ with $g_{0 S}=g_{0 S}^{\prime}$ and $g_{1 S}=g_{1 S}^{\prime}=$

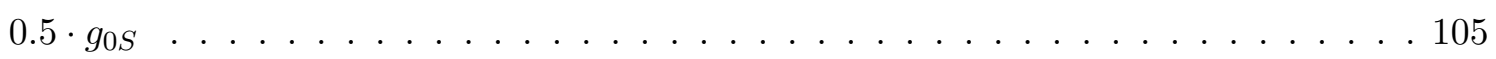

6.6 Contour plots of $\log _{10} B(\mu \rightarrow e \gamma)$ and $\log _{10} \Delta a_{\mu}$ with $g_{0 S}=g_{0 S}^{\prime}=g_{1 S}=g_{1 S}^{\prime} \ldots 106$

6.7 Contour plots of $\log _{10} B(\mu \rightarrow e \gamma)$ and $\log _{10} \Delta a_{\mu}$ with $g_{0 S}=g_{0 S}^{\prime}=0$ and $g_{1 S}=g_{1 S}^{\prime} .107$

6.8 One-loop induced Feynman diagrams from photon and $Z$ boson exchanges for $\mu-e$ conversion in electroweak-scale $\nu_{R}$ model. . . . . . . . . . . . . . . . . . . . . . . 114

6.9 Contour plots of $\log _{10} B(\mu-e$ conversion $)$ on the $\left(g_{0 S}, M_{\text {mirror }}\right)$ plane $(\mathrm{NH}+$ Scenario

1) with $g_{0 S}=g_{0 S}^{\prime}$ and $g_{1 S}=g_{1 S}^{\prime}=10^{-2} g_{0 S} \ldots \ldots \ldots \ldots \ldots \ldots \ldots$

6.10 Contour plots of $\log _{10} B(\mu-e$ conversion $)$ on the $\left(g_{0 S}, M_{\text {mirror }}\right)$ plane $(\mathrm{NH}+$ Scenario

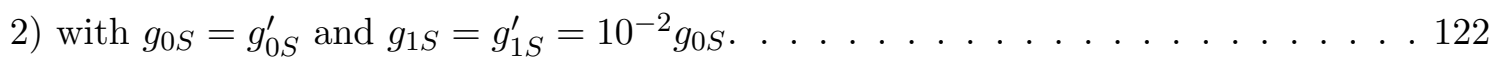

6.11 Contour plots of $\log _{10} B(\mu-e$ conversion $)$ on the $\left(g_{0 S}, M_{\text {mirror }}\right)$ plane $(\mathrm{NH}+$ Scenario

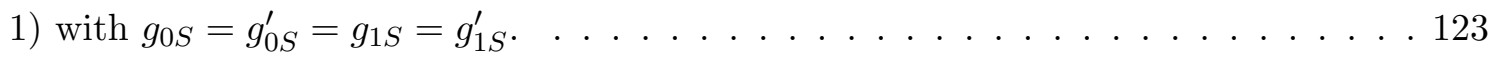

6.12 Contour plots of $\log _{10} B(\mu-e$ conversion $)$ on the $\left(g_{0 S}, M_{\text {mirror }}\right)$ plane $(\mathrm{NH}+$ Scenario

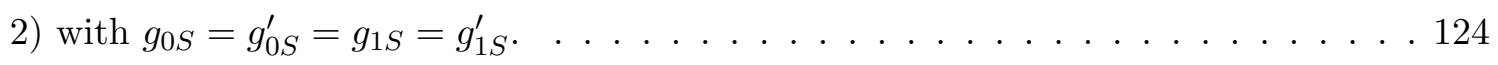

C.1 One-loop induced Feynman diagrams from photon and $Z$ boson exchanges for $\mu \rightarrow e$ conversion in EW-scale $\nu_{R}$ model. . . . . . . . . . . . . . . . . . . . . 154

C.2 One-loop induced Feynman diagrams Higgs exchange with heavy quarks and mirror

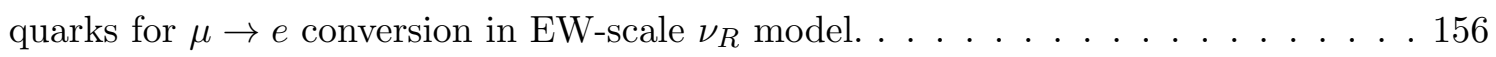

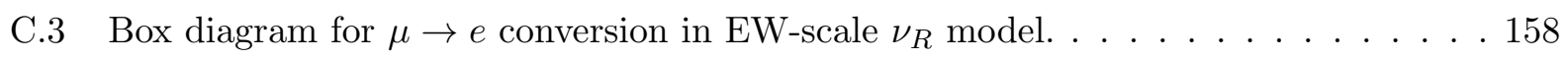




\section{Chapter 1}

\section{Introduction}

Over the past few decades, researches in particle physics field have focused on the socalled Standard Model (SM) which describes the universe in terms of Matter (fermions) and Force (bosons). The SM has served as a hardcore theory to understand and predict how particles and forces are related to each other. Over time it has provided excellent agreements with almost all experimental results. Furthermore the renormalizability of the SM made it possible to study the radiative or quantum corrections in a consistent manner. As an example, the electroweak corrections resulted from a theory that mixes the electromagnetic and weak contributions. Several illustrative example of electroweak corrections in the SM include unitarity of the Cabibbo-Kobayashi-Maskawa (CKM) matrix, prediction of the $W$ and $Z$ boson masses, top-quark mass and an estimation of the Higgs Boson mass. The year of 2012 witnessed the discovery of a new particle of mass $126 \mathrm{GeV}$ observed by the ATLAS and CMS experiments at the Large Hadron Collider (LHC). The new particle is said to be compatible with Higgs boson since it has behaved, interacted and decayed as in the predictions of the SM. Additionally, leptonic family numbers are accidentally conserved quantities in the SM regarding the fact that neutrinos are massless.

However this is not the end of the story. If the $126 \mathrm{GeV}$ object is the "end of the road" then the SM turns out to be incomplete due to several major unsolved problems: 
- We do not know whether the new $126 \mathrm{GeV}$ particle is the long-sought-after SM Higgs boson or an impostor. There could be also other types of Higgs bosons which SM could not predict.

- Typical concerns about the origin of mass hierarchy and mixings: "Why are there three generations of quarks and leptons?", "Why exist very different mass scales of quark and lepton sectors?".

- The neutrino oscillations resulting in non-zero neutrino masses have been observed. It was raising a question about the origin of neutrino mass and a possible explanation of why they are so light.

- Evidences of neutrino oscillations also show that the conservation law for leptonic family numbers is invoked. However the total lepton number must be conserved in the SM, which gives the possibility to see rare muon decays such as $\mu \rightarrow e \gamma$ or $\mu N \rightarrow e N$.

- The unification of gravity with the other three fundamental forces. So far we have been omitting its existence.

- There are other important questions remaining unrevealed, for example, "What is dark matter?", "Matter-antimatter asymmetry: What happened to the antimatter after the big bang?".

This lack of attention is significant because not solving these questions will incur an incomplete picture of the subatomic world, which will affect any future theoretical and experimental developments of particle physics field.

In order to address the above problems we apparently need to go search for new physics beyond the SM (BSM). It refers to theoretical developments needed to explain the deficiencies of the SM. Upon the addressed unsatisfactory situation, windows into BSM can be neutrino masses and lepton flavor violating processes such as $\mu \rightarrow e \gamma, \mu-e$ conversion. It is noticed that radiative 
decay of the muon into electron is possible but with an unobservable rate highly suppressed with the minuscule neutrino masses. Searches for lepton flavor violating rare processes in high intensity experiments are thus important for new physics beyond the SM. They all can potentially be the Great Guide to the road ahead.

In the light of this road guidance, our research has constructed a "Model of Neutrino Masses and Mixings" within the framework of the so-called "Electroweak-scale right-handed neutrino" $\left(\mathrm{EW}-\nu_{R}\right)$ model which has basically proposed a possibility of producing and detecting right handed neutrinos at the colliders. In the model of neutrino masses and mixings the experimentally desired form of the Pontecorvo-Maki-Nakagawa-Sakata (PMNS) matrix is obtained by applying an $A_{4}$ symmetry to the Higgs singlet sector responsible for the neutrino Dirac mass matrix. This mechanism naturally avoids potential conflict with the LHC data which severely constrains the Higgs sector, in particular the Higgs doublets. Moreover, by making a simple ansätz we are able to further explore the mass matrices for charged lepton and quark sectors. This might contribute to the understanding of the very distinctive mass structures of leptons and quarks. Interesting outcomes of this model of neutrino masses and mixings are analyses of muon rare decays: $\mu \rightarrow e \gamma$ and $\mu-e$ conversion in which we have incorporated all current experimental limits and projected sensitivities used to put constraints on the parameter space of the model. Furthermore we also have clues to help narrow down searched regions for near future experiments.

All detailed discussions and results of our research will be presented in this thesis. Let us first introduce the road map to the thesis.

- Chapter 2 will give us a brief summary of the SM's particle content and gauge interactions. The so-called Higgs mechanism generating masses for fermions and bosons and resulting in mixing matrices is also discussed.

- Chapter 3 will walk us through neutrinos masses and mixings, two types of neutrino mass 
which are Dirac and Majorana. Moreover the well-known seesaw mechanisms generically modeling the observed neutrino masses $\sim \mathcal{O}(e V)$ will be briefly reviewed.

- Chapter 4 will totally focus on the EW- $\nu_{R}$ model. It includes step-by-step model building process, the model's possible signature and phenomenological constraints.

- Chapter 5 will present the full context of the model of neutrino masses and mixings. Besides, we also provide a possible explanation on why the PMNS matrix is very different from the CKM matrix.

- We dedicate Chapter 6 to perform updated analyses for the one-loop induced lepton flavor violating radiative decays $l_{j} \rightarrow l_{i} \gamma$ and the muon-to-electron conversion in nuclei like aluminum, titanium and gold in the context of the model of neutrino masses and mixings introduced in the previous chapter.

- Chapter 7 will provide implications on quark mass matrices from the point of view of the neutrino model along with relevant assumptions.

- We conclude the thesis with Chapter 8. Useful formulas and calculations can be found in the Appendices.

With the brief indication of how the thesis will proceed, we are now ready to explore the model of neutrino masses and mixings and its phenomenological implications. 


\section{Chapter 2}

\section{Standard Model Overview}

In mid 1970s, particle physicists combined all knowledge about fundamental particles and forces or mediators which build up almost everything we have known so far into a theory called Standard Model (SM). Since then, the SM has provided extremely successful explanations for many vital observations and predicted a wide variety of phenomena. In the following sections, we will learn more about basic SM and its key role in understanding of the phenomenology of particle physics.

\subsection{Particle families and their interactions}

Fundamental particles are building blocks of matter, called fermions, and the mediators of interactions, called bosons.

Fermions can make up ordinary matter which is needed to make an atom, or exotic matter produced in high energy collisions, or very exotic matter produced in very high energy collisions. Fermions obey Fermi-Dirac statistics [1] and have spin 1/2. They come in one of twelve flavors and are organized in three families with identical quantum number but different masses. The elementary

fermions are either quarks or leptons, and they are differentiated by their charges under strong and 
electromagnetic interactions. For example, let us take the first family which contains

$$
\text { Quark: } u, d \text { and Lepton: } \nu_{e}, e \text {. }
$$

Two quarks carry so-called color charges under the strong interactions but two leptons do not. Under the electromagnetic interaction, two quarks have charges $2 / 3$ (for the up quark) and $1 / 3$ (for the down quark) while two leptons have charges 0 (for the electron neutrino) and -1 (for the electron). Either quarks or leptons come in one of six flavors

- Quarks: u, c, t; d, s, b1,

- Leptons: e, $\mu, \tau, \nu_{e}, \nu_{\mu}, \nu_{\tau}$.

Another difference between quarks and leptons is that quarks are always bound to other quarks by the strong force whereas heavy leptons $(e, \mu, \tau)$ can be found free or bound to other charged fermions by the electromagnetic force and neutrinos only interact with themselves and other particles via the weak force. Masses of these fermions can fall into a range from the sub-eV neutrino masses to the $172 \mathrm{GeV}$ top mass [3]. Moreover, each family shows such a peculiar mass structure in which the masses are hierarchically separated from one another. A massive charged fermion, e.g. electron has two possible chiralities called left-handed and right-handed. In the case of neutrinos, only the left-handed chirality has been observed so far and this seems to agree well with SM's prediction because neutrinos are massless in SM. Further discussion on neutrinos and their chiralities will be provided in the next chapter. Quarks are known to bind into triplets and doublets. The triplets are called baryons and the doublets (quark-antiquark pair) are called mesons. Collectively baryons (the heavy triplets), mesons (the middleweight doublets), and quarks (the fundamental particles) are known as hadrons.

In contrast to fermions, bosons obey Bose-Einstein statistics [2] and have integral spin quantum numbers $( \pm 0, \pm 1, \pm 2, \ldots)$. The SM interactions are characterized by four vector bosons or gauge

\footnotetext{
${ }^{1}$ Sometimes we refer $\mathrm{u}, \mathrm{c}, \mathrm{t}$ as up-quark sector and $\mathrm{d}, \mathrm{s}, \mathrm{b}$ as down-quark sector
} 
bosons of spin 1 . The photon carries the electromagnetic force between particles with charge, the gluon strong force between particles with color (quarks and gluons), the Z and W's weak force between certain particles with flavor. It is noticed that the photon and gluons are massless while the $\mathrm{Z}$ and W's bosons are massive. This explains why weak interactions are weak at low energy because of a suppression of $1 / M_{Z, W}$ factor. However, they still stand out because of their violations in universal symmetries such as parity $P$, charge conjugation $C, C P$, time-reversal $T$, and lepton/baryon $(L / B)$ number, which all are conserved under the electromagnetic and strong interactions. Another boson we should mention about is the scalar Higgs boson ( $\operatorname{spin}=0$ ) which was discovered in 2012 [4,5] and the mediator of the Higgs mechanism. Within the frame work of the mechanism, Higgs is able to give mass to all particles and itself. Signals from the LHC show that Higgs has mass around $125 \mathrm{GeV}[6]$ but we do not know whether or not it is the SM Higgs. Future experiments carried out at the colliders will hopefully give us the answer soon. A further discussion about Higgs mechanism will be given in section 2.3. A summary of SM particle content is given in Fig. 2.1.

\subsection{The SM gauge interactions}

\subsubsection{Gauge transformations}

Gauge transformation or more precisely, local gauge transformation when applied to a local symmetry will result in the representation of the symmetry group that is a function of the manifold and can be taken to act differently on different points of spacetime. This section is arranged as follows. We first review a physical quantity needed for a symmetry to work on. This quantity is a Lagrangian of a theory. We then discuss the role of symmetry and two kind of symmetries called global and local symmetry. Finally, we will have a heavy discussion on local symmetry and local gauge transformation in quantum electrodynamics (QED) and then generalize a renormalizable gauge theory at the end of this section. 


\section{The Standard Model}

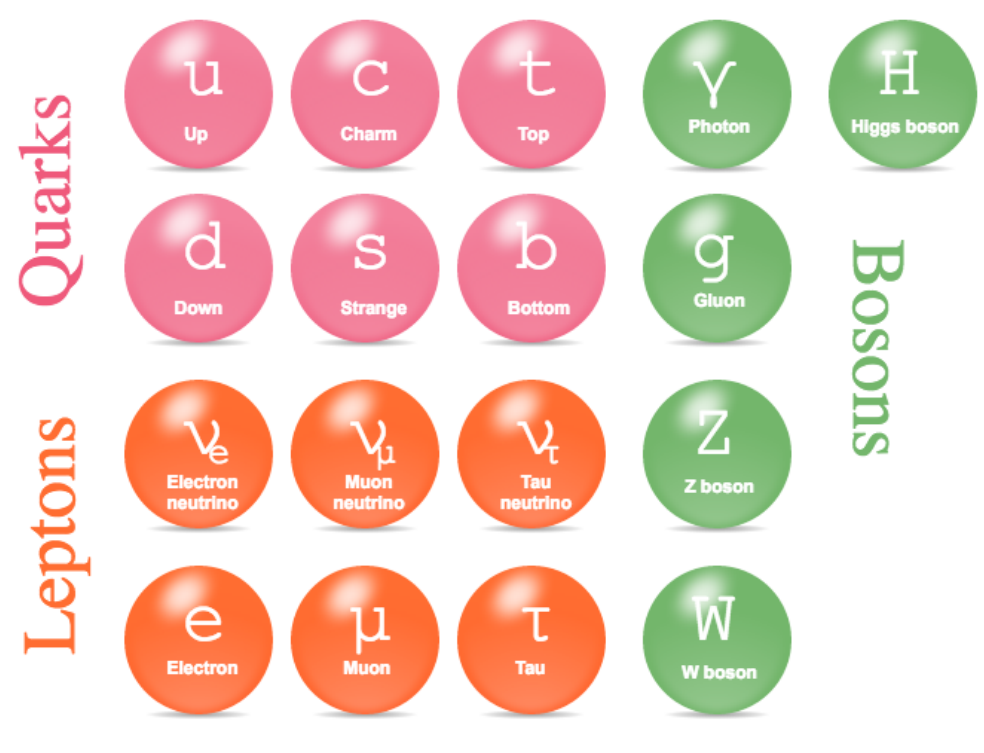

$125 \mathrm{GeV}$ Higgs boson(SM?)

Figure 2.1: The Standard Model particle content

To get the idea of a Lagrangian, let us start with a quantum field theory (QFT) basis in which particles are related to fields $\phi(x)$ in the space-time coordinates. The fundamental quantity of the field theory is the action, $S$, which can be written as

$$
S=\int L d t=\int \mathcal{L}(x) d^{4} x, \quad \mathcal{L}(x)=\sum_{i} c_{i} \mathcal{O}_{i}(x)
$$

where $\mathcal{L}$ is the Lagrangian density ${ }^{2}$ which is a function of fields $\phi(x)$ and their derivatives $\partial_{\mu} \phi$. Although there is no strict form for a Lagrangian, a typical one will contain terms bilinear in the fields called free Lagrangian, $\mathcal{L}_{\text {free }}$ and terms called interaction Lagrangian $\mathcal{L}_{\text {int }}$. Let us take an example of the so-called " $\phi 4$ theory" whose Lagrangian is

$$
\mathcal{L}=\frac{1}{2}\left(\partial_{\mu} \phi\right)^{2}-\frac{1}{2} m^{2} \phi^{2}-\frac{\lambda}{4 !} \phi^{4}
$$

The interaction term $\mathcal{L}_{\text {int }}=-\frac{\lambda}{4 !} \phi^{4}$ accounts for field interactions. In order to see how a corresponding interaction behaves we must consider the dimension of the coefficients $c_{i}$ in Eq. (2.2).

\footnotetext{
${ }^{2}$ Hereinafter we will refer to $\mathcal{L}$ simply as the Lagrangian.
} 
For example, a term with negative dimension, i.e. $c=1 / \Lambda^{r}$ where $r>0$ and $\Lambda$ is the energy scale will appear as "non-renormalizable interaction". According to the QFT, such an interaction is not applicable at low energy scale but may cause a potential interest at high energy where they make the theory incalculable. Therefore, non-renormalizable terms are only relevant in an effective theory having a cutoff energy scale $(\Lambda)$.

Symmetry plays a crucial role in outlining the independent regularities of the laws of nature in the context of specific dynamics. Within the scope of this thesis, we will be considering three popular symmetry groups: $U(1)$ and $S U(2), S U(3)$ which are special cases of the general $S U(N)$ group. We start with an infinitesimal group element $g$

$$
g(\alpha)=1+i \alpha^{a} T^{a}+\mathcal{O}\left(\alpha^{2}\right)
$$

where $T^{a}$ are the generators of the symmetry group. The set of generators will have the commutation relations

$$
\left[T^{a}, T^{b}\right]=i f^{a b c} T^{c}, f^{a b c} \text { are structure constants }
$$

which form a so-called Lie Algebra and the identities

$$
\left[T^{a},\left[T^{b}, T^{c}\right]\right]+\left[T^{b},\left[T^{c}, T^{a}\right]\right]+\left[T^{c},\left[T^{a}, T^{b}\right]\right]=0 .
$$

A group which has one of the generators $T^{a}$ commutes with the others and phase rotations $\psi \rightarrow e^{i \alpha} \psi$ is called $U(1)$. A subgroup of $U(1)$ which commutes with other unitarity transformations is known as $S U(N)$. The generators of $S U(N), t^{a}$, are Hermitian traceless matrices. In the case of $N=2$, generators of $S U(2)$ are chosen to be

$$
t_{a}=\sigma_{a} / 2, a=1,2,3, \quad \sigma_{a} \text { are Pauli matrices }
$$

In the case of $N=3$, generators of $S U(3)$ are

$$
t_{A}=\lambda_{A} / 2, A=1,2 \ldots 8, \quad \lambda_{A} \text { are Gell-Mann matrices }
$$

If a Lagrangian under a transformation on the fields $\psi$

$$
\psi_{i}(x) \rightarrow U_{i j} \psi_{j}(x)
$$


is invariant, we then call this transformation a symmetry of the Lagrangian. From $(2.9)$ if $U$ does not depend on space-time point $x$, the symmetry is called global symmetry, otherwise, it called local symmetry. An example of global symmetry is $S U(2)$ isospin which acts on the quark doublet $Q=(u, d)^{T}$. Within the framework of quantum chromodynamics (QCD) with the limit $m_{u, d} \rightarrow 0$, the isospin transformation $Q \rightarrow U Q$ is a symmetry of QCD Lagrangian for $u, d$. In other words, isospin symmetry is given by the invariance of the Hamiltonian of the strong interactions under the action of the Lie group $\mathrm{SU}(2)$.

Gauge symmetries are the invariances of the Lagrangians under gauge transformations which are realized on the fields as a local phase rotation. A celebrated example is the famous gauge symmetry of QED whose Lagrangian is written as

$$
\mathcal{L}_{Q E D}=\bar{\psi}\left(i \gamma^{\mu} \partial_{\mu}-m\right) \psi-\frac{1}{4} F_{\mu \nu} F^{\mu \nu}-e \bar{\psi} \gamma^{\mu} \psi A_{\mu}
$$

where the electromagnetic field $F_{\mu \nu}$ is expressed in terms of the vector potential $A_{\mu}$ as $F_{\mu \nu}=$ $\partial_{\mu} A_{\nu}-\partial_{\nu} A_{\mu}$. In this case, the fields are the complex-valued Dirac. Under gauge transformation

$$
\psi(x) \rightarrow e^{i \alpha(x)} \psi(x), A_{\mu}(x) \rightarrow A_{\mu}(x)-\frac{1}{e} \partial_{\mu} \alpha(x)
$$

in which an angle $\alpha(x)$ varies arbitrarily from point to point, our Lagrangian 2.10 must be invariant. In fact, the QED Lagrangian can be simplified by introducing the gauge covariant derivative

$$
D_{\mu} \equiv \partial_{\mu}+i e A_{\mu}(x)
$$

that can be transformed according to Eq. (2.11)

$$
\begin{aligned}
D_{\mu} \psi(x) & \rightarrow\left[\partial_{\mu}+i e\left(A_{\mu}-\frac{1}{e} \partial_{\mu} \alpha\right)\right] e^{i \alpha(x)} \psi(x) \\
& =e^{i \alpha(x)}\left(\partial_{\mu}+i e A_{\mu}\right) \psi(x)=e^{i \alpha(x)} D_{\mu} \psi(x)
\end{aligned}
$$

Therefore, the QED Lagrangian is simply

$$
\mathcal{L}_{Q E D}=\bar{\psi}\left(i \gamma^{\mu} D_{\mu}-m\right) \psi-\frac{1}{4} F_{\mu \nu} F^{\mu \nu}
$$


The first term is clearly invariant based on the above argument and the kinetic term $F_{\mu \nu} F^{\mu \nu}$ is also invariant as the vector field $A_{\mu}$ transforms in (2.11). In conclusion, we are able to prove that the QED Lagrangian follows from the principle of gauge invariance under local U(1) transformations. In addition, gauge invariance shows us why the photon is massless, since a mass term $A_{\mu} A^{\mu}$ would break gauge invariance.

Finally, we will present the steps to construct a general renormalizable gauge theory of spin $0,1 / 2,1$ fields $\Phi$.

- Choose a basis of generators $t_{a}$ for a compact gauge group $G$ with associated real vector field, $A_{\mu}^{a}$ and field strength $F_{\mu \nu}^{a}=\partial_{\mu} A_{\nu}^{a}-\partial_{\nu} A_{\mu}^{a}-g f^{a b c} A_{\mu}^{b} A_{\nu}^{c}$.

- Define a covariant derivative $D_{\mu}=\partial_{\mu}+i g A_{\mu}^{a} T_{a}$ where $T_{a}$ is the action of the generator $t_{a}$ on the fields $\Phi$.

- Make a gauge transformation of the fields $\Phi(x) \rightarrow U(x) \Phi(x)$ and vector fields so that $D_{\mu}(U(x) \Phi(x)) \rightarrow U(x)\left(D_{\mu} \Phi(x)\right)$ and $F_{\mu \nu}^{a} T_{a} \rightarrow U(x)\left(F_{\mu \nu}^{a} T_{a}\right) U^{\dagger}(x)$.

- Write a corresponding gauge Lagrangian, for example,

$$
\mathcal{L}=-\frac{1}{4}\left(F_{\mu \nu}^{a}\right)^{2}+\frac{1}{2}\left(D_{\mu} \Phi\right)^{T} D^{\mu} \Phi-\frac{1}{2} m^{2} \Phi^{T} \Phi
$$

where the first term describes the famous Yang-Mills gauge theory, the other terms construct the locally gauge invariant Lagrangian.

\subsubsection{Gauging SM interactions}

SM itself is a gauge theory so in order to define it we have to identify key ingredients listed in the previous section. As mentioned in the section 2.1. SM describes three interactions related to electromagnetic, strong and weak forces. Therefore, it is reasonable to develop a gauge group 
for SM which contains all generators of groups describing the above interactions. So far we have known that the QED and QCD detail the electromagnetic and strong interactions and then involve the $U(1)_{e m}$ and $S U(3)_{c}$ ('c' stands for 'color') gauge groups, respectively. It is important to figure out which gauge group represents the weak interaction as well. Because parity is violated in weak interactions as opposed to electromagnetic and strong interactions, one expects to look at Dirac spinors in a chiral view. If we take the first family fermions $(\nu, e, u, d)$ for consideration, they have to be split into left- and right-handed components: $\nu_{L}, e_{L}, e_{R}, u_{L}, u_{R}, d_{L}$, and $d_{R}{ }^{3}$.

We shall now discuss weak interactions before gauge theory comes along. In 1950s, it is suggested that an effective Lagrangian for weak interactions

$$
\mathcal{L}_{\text {eff }}(x)=-\frac{G_{F}}{\sqrt{2}} J_{W, \mu}^{\dagger}(x) J_{W}^{\mu}(x)+\text { h.c. }
$$

with the Fermi coupling constant, $G_{F} \simeq 10^{-5} / m_{\text {proton }}^{2}$ and the weak current $J_{W}^{\mu}(x)$ having the V-A form

$$
J_{W}^{\mu}=\bar{\nu}_{L} \gamma^{\mu} e_{L}+\bar{u}_{L} \gamma^{\mu} d_{L}, \text { note that } \psi_{L}=\frac{1-\gamma^{5}}{2} \psi
$$

It is said that the Lagrangian (2.16) described successfully parity violating and charge changing weak interaction. Nevertheless it is lack of renormalizability because of the negative dimension of the coefficient $G_{F}\left([\text { energy }]^{-2}\right)$ and violates unitarity at high energies, even in the lowest order of $G_{F}[7]$.

\subsubsection{Unitarity violation and bad high energy behavior}

We have known that at tree level measured cross-sections of processes such as $n \rightarrow p e^{-} \bar{\nu}_{e}$ or $\nu_{\mu} e^{-} \rightarrow \mu^{-} \nu_{e}$ turn out to agree very well with the theory containing the above effective Lagrangian. However when considering, for example, one-loop process $\nu_{\mu} e \rightarrow \nu_{\mu} e$ as shown in Fig. 2.2 one gets an infinite cross-section. This "disease" has a deep root in the violation of tree-level unitarity. In quantum physics, unitarity restricts the probability from exceeding 1 . Let us take an example

\footnotetext{
${ }^{3}$ The right-handed neutrino $\nu_{R}$ is not presented here since it does not exist in the SM
} 


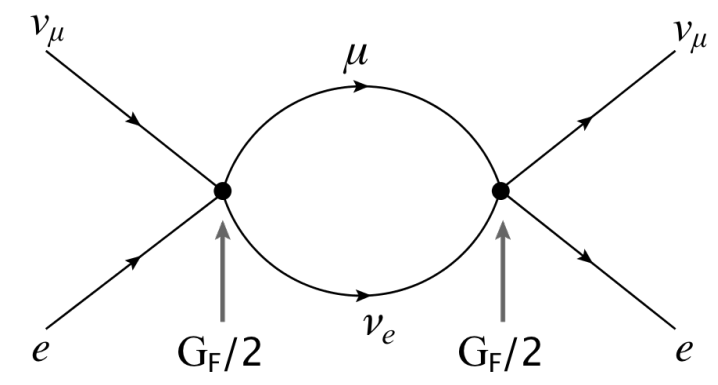

Figure 2.2: One-loop process $\nu_{\mu} e \rightarrow \nu_{\mu} e$ results in an infinite cross-section.

of scattering theories with the $S$-matrix defined as

$$
S=\mathbb{I}+i T
$$

where $\mathbb{I}$ indicated "no scattering" and $T$ is the scattering matrix. Unitarity, in this case, requires sum of probabilities

$$
S S^{\dagger}=S^{\dagger} S \leq 1
$$

If one considers the interaction of $\nu_{\mu} e^{-} \rightarrow \mu^{-} \nu_{e}$ in Fig. (2.2) its amplitude has only the $J=1$ partial wave and the cross-section goes like

$$
\sigma \sim G_{F}^{2} s
$$

where $s=2 m_{e} E_{\nu}, E_{\nu}$ is the $\nu_{\mu}$ energy in the lab frame. According to the scattering theory, the cross-section can be expressed as

$$
\sigma \sim \frac{\left|S_{J=1}\right|^{2}}{s}
$$

Since unitarity forces $\left|S_{J=1}\right|^{2} \leq 1$ the cross-section, $\sigma_{J=1}$, is bounded by $s^{-1}$. Therefore

$$
G_{F}^{2} s \leq \frac{1}{s} \Rightarrow \sqrt{s} \leq G_{F}{ }^{-1 / 2} \sim 300 \mathrm{GeV}
$$

This means the weak interaction's unitarity is violated if one goes beyond $300 \mathrm{GeV}$. Actually the broken energy scale is about $1000 \mathrm{GeV}$ to a good precision. How could one avoid such a bad high energy behavior from occurring? 


\subsubsection{Problem solvers $-W^{ \pm}$and $W^{0}$}

It has been known that QED is described by the Lagrangian

$$
\mathcal{L}_{Q E D}=e J_{E M}^{\mu} A_{\mu}
$$

where $J_{E M}^{\mu}$ is the electromagnetic current, $A_{\mu}$ is a photon field and $e$ - the electron's charge - is the dimensionless coupling constant of the electromagnetic interaction. QED has shown a good high energy behavior and contained no infinities at higher orders in perturbation theory. Here is why. All the infinities in QED can be eliminated via "renormalization" triggered by the dimensionless coupling constant $e$. It is worth noticing that the electromagnetic interaction mediated by the spin-1 vector photon field makes it possible to have the dimensionless coupling.

Therefore it would be a good idea if we try to mimic QED in order to cure the infinities and unitarity violation problems in weak interactions. Let us suggest a choice of charged vector bosons (spin 1), $W_{\mu}^{ \pm}$and write down the weak Lagrangian in the same manner with that of $\mathrm{QED}$ as

$$
\mathcal{L}_{\text {weak }}=g J_{W}^{\mu} W_{\mu}
$$

where $J_{W}^{\mu}$ is introduced in Eq. (2.17) and $g$ is the dimensionless coupling constant of the weak interaction. We can generate the effective interaction by a massive gauge boson as shown in Fig. (2.3) and have such an identification

$$
G_{F}=\frac{\sqrt{2}}{8} \frac{g^{2}}{M_{W}^{2}}
$$

in the case of the squared transferred momentum, $q^{2} \ll M_{W}^{2}$.

We now investigate the problems of unitarity and renormalizability in the theory involving intermediate vector boson $W^{ \pm}$. Unfortunately the bad high energy behavior remains, for instance, in a process $\nu_{e} \bar{\nu}_{e} \rightarrow W^{+} W^{-}$. Since $W^{ \pm}$are massive spin-1 fields they have 3 degrees of freedom of polarizations: 2 transverse and 1 longitudinal. The longitudinal polarized $W$, denoted as $W_{l}$, has 

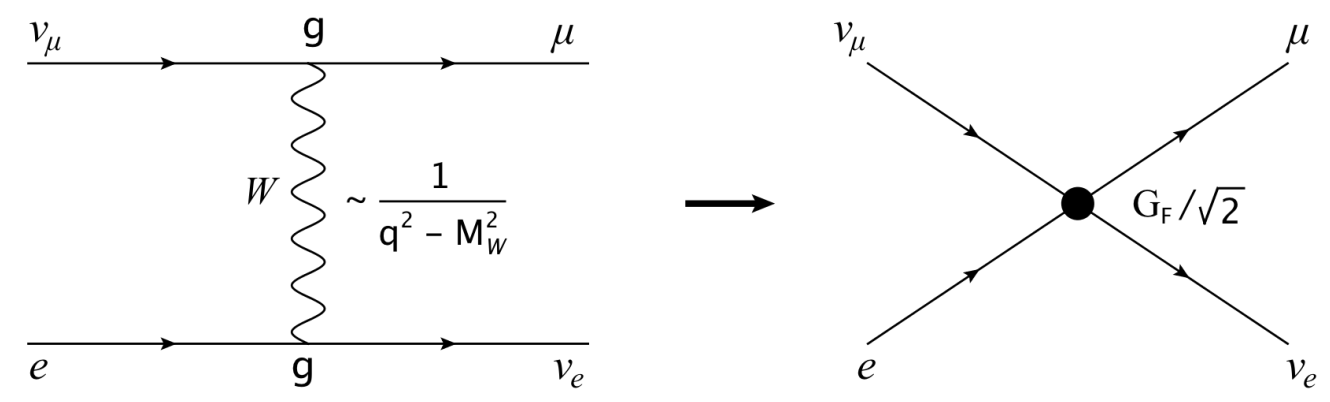

Figure 2.3: $e \nu_{\mu} \rightarrow \mu \nu_{e}$ process with $W$ exchange approximation to four-fermion interaction at low energies.

a polarization of the form

$$
\varepsilon_{\mu}^{(3)}=\frac{k_{\mu}}{M_{W}}+\mathcal{O}\left(\frac{M_{W}}{E}\right)
$$

where $E$ is the energy in the center-of-mass frame. Therefore the cross-section of a diagram shown in Fig. (2.4) contains

$$
\sum_{\text {polarization }} \varepsilon_{\mu} \varepsilon_{\nu}^{*}=-g_{\mu \nu}+\frac{k_{\mu} k_{\nu}}{M_{W}^{2}} .
$$

The second term causes the bad energy behavior that we will see later. The amplitude, therefore, is given by

$$
\begin{aligned}
f_{t} & =-2 g^{2} \bar{v}\left(p^{\prime}\right) \frac{\notin(\not p-\not k) \notin\left(1-\gamma_{5}\right)}{(p-k)^{2}-m_{e}^{2}} u(p) \\
& \simeq-\frac{2 g^{2}}{k^{2}-2 p \cdot k} \bar{v}\left(p^{\prime}\right)\left(\not k^{\prime} / M_{W}\right)(\not p-\not k)\left(\not k / M_{W}\right)\left(1-\gamma_{5}\right) u(p) \\
& \simeq \frac{2 g^{2}}{M_{W}^{2}} \bar{v}\left(p^{\prime}\right) \not k^{\prime}\left(1-\gamma_{5}\right) u(p) .
\end{aligned}
$$

One can choose the following momentum configuration

$$
\begin{array}{ll}
p_{\mu}=(E, 0,0, E), & p_{\mu}^{\prime}=(E, 0,0,-E) \\
k_{\mu}=(E, k \mathbf{e}), & k_{\mu}^{\prime}=(E,-k \mathbf{e})
\end{array}
$$

where $\mathbf{e}=(\sin \theta, 0, \cos \theta)$. It is proved that the cross-section

$$
\sigma_{t} \approx \frac{G_{F}^{2} E^{2}}{3 \pi}
$$




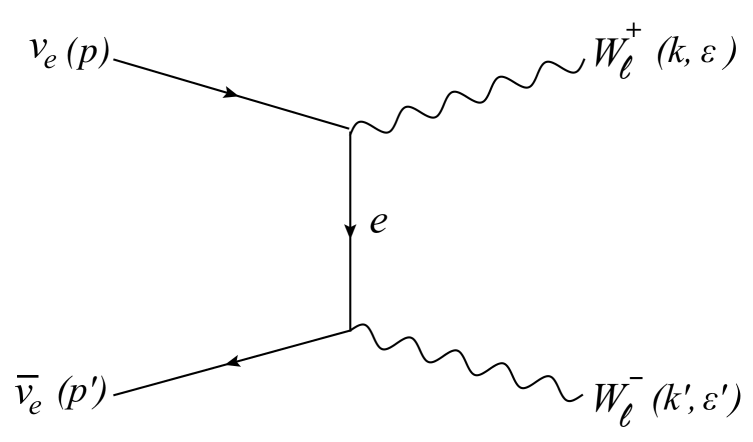

Figure 2.4: $\quad \nu_{e} \bar{\nu}_{e} \rightarrow W^{+} W^{-}$process with a t-channel.

On the other hand, from the partial-wave expansion [11, the cross-section is given by

$$
\sigma_{t}=\frac{\pi}{E^{2}} \sum_{J}(2 J+1)\left|S_{\lambda_{3}, \lambda_{4} ; \lambda_{1}, \lambda_{2}}^{J}\right|^{2}
$$

where $\lambda_{1}=-\lambda_{2}=1 / 2$ are the helicities of $\nu_{e}, \bar{\nu}_{e}$ and $\lambda_{3}=\lambda_{4}=0$ are the helicities of $W^{ \pm}$. This cross-section comes from a pure $J=1$ partial wave. From Eqs. 2.30, 2.31) and the unitarity constraint we have

$$
\frac{G_{F}^{2} E^{4}}{9 \pi^{2}}=\left|S_{0,0 ; 1 / 2,-1 / 2}^{J=1}\right|^{2} \leq 1
$$

or

$$
G_{F} s \leq 12 \pi \quad \text { where } s \equiv 4 E^{2} .
$$

This implies that the unitarity is violated at $\sqrt{s}=2 E \sim 1800 \mathrm{GeV}$ or $E \sim 900 \mathrm{GeV}$.

Although the coupling $g$ is now dimensionless which helps fix the infinities problem, the theory with intermediate vector bosons $W^{ \pm}$is still not renormalizable. According to the free massive vector boson Lagrangian

$$
\mathcal{L}_{W}=-\frac{1}{4} F_{\mu \nu} F^{\mu \nu}+M_{W}^{2} W_{\mu}^{\dagger} W^{\mu}
$$

one can find the propagator in momentum space as

$$
i \Delta_{\mu \nu}(k)=-i \frac{g_{\mu \nu}-k_{\mu} k_{\nu} / M_{W}^{2}}{k^{2}-M_{W}^{2}+i \epsilon} .
$$


As $k \rightarrow \infty$, the propagator acts like a constant and the interaction is not renormalizable because of power counting. Therefore something must come rescue this theory. It turns out that in order to cancel the bad high energy behavior, one can implement other diagrams for the above interaction. They are either s-channel or u-channel exchange diagrams as shown in Fig. (2.5). For the purpose of this review we only consider the choice of neutral vector boson exchange or the s-channel diagram in Fig. 2.5 -(b). It is noticed that the couplings $f, f^{\prime}$ obey the symmetry of a Lie group forming

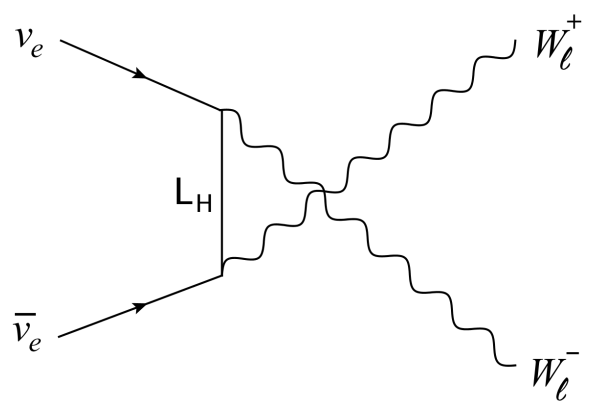

(a)

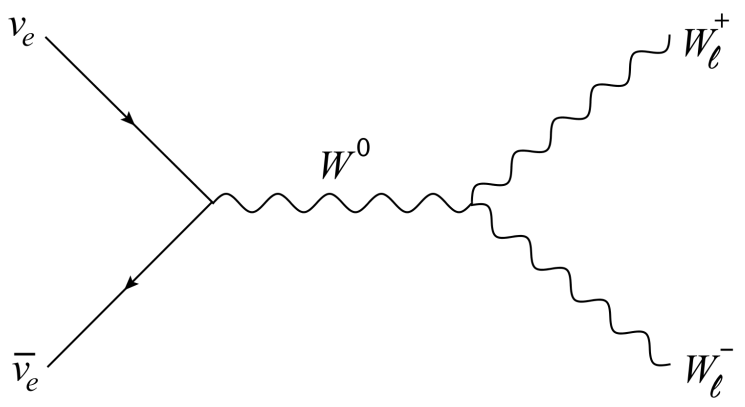

(b)

Figure 2.5: $\quad \nu_{e} \bar{\nu}_{e} \rightarrow W^{+} W^{-}$with (a) u-channel and (b) s-channel.

the Yang-Mills theory. The amplitude is proven to be

$$
f_{s} \simeq \frac{-f f^{\prime}}{M_{W}^{2}} \bar{v}\left(p^{\prime}\right) \not k^{\prime}\left(1-\gamma_{5}\right) u(p)
$$

with a choice of $W^{0} W^{+} W^{-}$coupling to have the Yang-Mills structure. If

$$
f f^{\prime}=2 g^{2}
$$

then $f_{s}$ will cancel the bad high energy behavior from Eq. (2.29). In a nutshell, the good high energy behavior of the weak interactions forces us to introduce $W^{0}$, in addition to $W^{ \pm}$, with the couplings obeying the symmetry of $S U(2)$ and being universal coupling $g$. 


\subsubsection{SM gauge group, $G_{S M}$}

If we introduce the left-handed fermion doublets

$$
l_{L} \equiv\left(\begin{array}{c}
\nu_{L} \\
e_{L}
\end{array}\right), \quad q_{L} \equiv\left(\begin{array}{c}
u_{L} \\
d_{L}
\end{array}\right),
$$

and then define $W_{\mu}^{ \pm}$in terms of real vectors $W_{\mu}^{1,2}$ as

$$
W_{\mu}^{ \pm} \equiv \frac{\left(W_{\mu}^{1} \mp i W_{\mu}^{2}\right)}{\sqrt{2}}
$$

the Lagrangian (2.24) can be written as

$$
\mathcal{L}_{\text {weak }}=g \bar{l}_{L} \gamma^{\mu}\left(W_{\mu}^{1} T_{1}+W_{\mu}^{2} T_{2}\right) l_{L}+g \bar{q}_{L} \gamma^{\mu}\left(W_{\mu}^{1} T_{1}+W_{\mu}^{2} T_{2}\right) q_{L}
$$

where $T_{1,2}=\sigma_{1,2} / 2$. We can see that $T_{1}$ and $T_{2}$ are the generators of gauge theory. In order to form a Lie algebra such that $\left[T_{1}, T_{2}\right]=i T_{3}, T_{3}=\sigma_{3} / 2$, we also have $T_{3}$ as a generator corresponding to a third real gauge boson, $W_{\mu}^{3}$. As a result, this predicts an additional neutral interaction. With the identification of 3 generators $T_{1,2,3}$ we can conclude that $S U(2)_{L}$ ('L' stands for 'left-handed' field components) is a part of the gauge group, $G_{S M}$.

As argued in the beginning of this section, $G_{S M}$ should also include generators of $U(1)$ and $S U(3)_{c}$. We have already seen the generators of the $S U(3)$ group in section 2.2.1 so I will not repeat them here. Instead we would focus on finding the generators of $U(1)$. As we have known, $U(1)$ associates with an electric charge generator called $Q$. However, we observe that $Q-T_{3}$ also

gives the same quantum number to all members of $S U(2)$ fermion doublets in 2.38 . In fact, one can go further by checking the commutation of this linear combination generator with other $S U(2)$ generators and confirm that

$$
\left[Q-T_{3}, T_{i}\right]=0, i=1,2,3
$$

We then define "hypercharge" generator of $U(1)$ as

$$
\frac{Y}{2} \equiv Q-T_{3} \quad \text { or } \quad Q=T_{3}+\frac{Y}{2} .
$$


The later one is known as Gell-Mann-Nishijima formula [8].

In conclusion, the gauge group of the SM is identified as

$$
G_{S M}=S U(3)_{c} \times S U(2)_{L} \times U(1)_{Y}
$$

in which $S U(2)_{L} \times U(1)_{Y}$ composes the electroweak group. Table 2.1) will summarize quantum number assignments of SM chiral fermions in the first family under gauge group, $G_{S M}$.

Table 2.1: Gauge quantum numbers of chiral SM fermions in the first family. Later on we can consider this as a generic notation for fermion families because the quantum numbers are the same for all three families

\begin{tabular}{||c|ccc||}
\hline & $S U(3)_{c}$ & $S U(2)_{L}$ & $U(1)_{Y}$ \\
\hline \hline$l_{L}$ & 1 & 2 & $-1 / 2$ \\
$e_{R}$ & 1 & 1 & -1 \\
$q_{L}$ & 3 & 2 & $1 / 6$ \\
$u_{R}$ & 3 & 1 & $2 / 3$ \\
$d_{R}$ & 3 & 1 & $-1 / 3$ \\
\hline
\end{tabular}

Before closing this section, let us discuss anomalies in gauge theories and why it is important to perceive anomaly cancellation. The presence of triangle diagrams which appear in the one-loop corrections to the tree-gauge-boson vertex is dangerous for a gauge theory because of the breakdown of gauge invariance presented by the so-called Ward identity. The anomalous term of a triangle diagram of three gauge bosons denoted as $A_{\mu}^{a}, A_{\nu}^{b}, A_{\lambda}^{c}$ (as shown in Fig. 2.6) ) is proportional to $\operatorname{Tr}\left[\left\{T_{a}, T_{b}\right\} T_{c}\right]$ which vanishes if there is no anomaly. To examine the anomalies of the SM gauge theory, it is convenient to start with the $S U(2)_{L} \times U(1)_{Y}$ gauge group and check to see if the anomalous terms cancel as required. As one may notice, three generators $\tau_{a}, \tau_{b}, \tau_{c}$ belongs to the $S U(2)_{L}$. They satisfy

$$
\operatorname{Tr}\left[\left\{\tau_{a}, \tau_{b}\right\} \tau_{c}\right]=\operatorname{Tr}\left[2 \delta_{a b} \tau_{c}\right]=0 .
$$

It is easy to show that the anomalies containing one $S U(3)$ or $S U(2)$ boson are zero because 


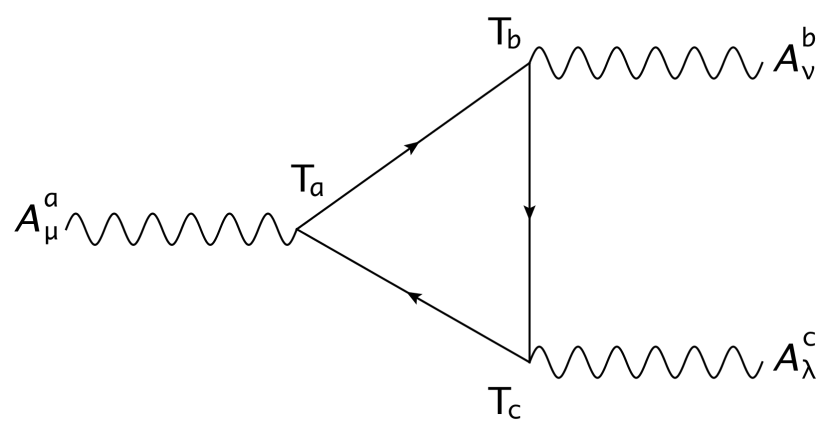

Figure 2.6: Anomalies from triangle diagrams in a gauge theory.

$$
\operatorname{Tr}\left[t_{a}\right]=0 \quad \text { or } \operatorname{Tr}\left[\tau_{a}\right]=0
$$

The anomaly of one $U(1)$ boson with two $S U(2)$ or $S U(3)$ boson is

$$
\operatorname{Tr}\left[\tau_{a}, \tau_{b} Y\right]=\frac{1}{2} \delta_{a b} \cdot \operatorname{Tr} Y \quad \text { or } \quad \operatorname{Tr}\left[t_{a}, t_{b} Y\right]=\frac{1}{2} \delta_{a b} \cdot \operatorname{Tr} Y,
$$

where $\operatorname{Tr} Y$ can be understood as the total of the sums running overs leptons and quarks. For each family, using the values of $Y / 2$ from Table (2.1) we have

$$
\begin{aligned}
\sum_{\text {lepton }} Y & =Y\left(e_{L}\right)+Y\left(\nu_{L}\right)+Y\left(e_{R}\right)=-1+(-1)+(-2)=-4 \\
\sum_{\text {quark }} Y & =N_{c} \cdot\left(Y\left(u_{L}\right)+Y\left(d_{L}\right)+Y\left(u_{R}\right)+Y\left(d_{R}\right)\right) \\
& =N_{c} \cdot\left(\frac{1}{3}+\frac{1}{3}+\frac{4}{3}+\left(-\frac{2}{3}\right)\right)=\frac{4}{3} N_{c} .
\end{aligned}
$$

where $N_{c}$ is the number of quark colors. Therefore,

$$
\operatorname{Tr} Y=\sum_{\text {lepton }} Y+\sum_{\text {quark }} Y=-4+\frac{4}{3} N_{c} .
$$

In order to cancel this anomaly (i.e., $\operatorname{Tr} Y=0$ ) one needs exactly three quark colors which turns out agree well with experimental data. Furthermore it is obvious that $\operatorname{Tr}\left[T_{3 L}\right]=0$. Since $Y=$ $2\left(Q-T_{3 L}\right)$ and $\operatorname{Tr} Y=0$ from the above argument, we obtain $\operatorname{Tr} Q=0$ which is verified by taking

$$
\sum_{\text {lepton }} Q+3 \cdot \sum_{\text {quark }} Q
$$

The SM theory is thus a chiral gauge theory which is completely anomaly-free. Nevertheless the anomalies cancellation entails that leptons and quarks in each family have to form a structure of doublets and singlets as $l_{L}, e_{R}, q_{L}, u_{R}, d_{R}$. 


\subsection{Higgs Mechanism and how to generate gauge boson masses}

In the theory of gauge invariance, vector gauge bosons such as $W^{ \pm}$are prevented from acquiring their masses in order to preserve the invariance. With the discovery of the mass of $W$-bosons to be about $80 \mathrm{GeV}$ 9], we may claim that the gauge symmetry has to be broken to give mass to gauge bosons as well as fermions. This is where "spontaneous symmetry breaking" (SSB) [10 takes off and becomes one of the most important mechanisms accounting for massive particles. The SSB can be applied to either global or local symmetry. However we will only focus on analyzing SSB of the local $U(1)$ gauge symmetry which leads us to the Higgs mechanism and that of the $S U(2)_{L}$ which helps us move toward SSB of SM weak interaction in the following section.

\subsubsection{Spontaneous symmetry breaking in $U(1)$ gauge theory}

Let us first start by introducing the Lagrangian of complex scalar fields $\phi$

$$
\mathcal{L}_{\text {gauge }}=-\frac{1}{4} F_{\mu \nu} F^{\mu \nu}+\left(D_{\mu} \phi\right)^{\dagger}\left(D^{\mu} \phi\right)-V(\phi)
$$

where $V(\phi)=-\mu^{2} \phi^{\dagger} \phi+\lambda\left(\phi^{\dagger} \phi\right)^{2}$. Recall that $D_{\mu}=\partial_{\mu}-i g A_{\mu}$. We can write $\phi$ in terms of 2 independent real components, $\phi_{1}$ and $\phi_{2}$ as

$$
\phi=\frac{1}{\sqrt{2}}\left(\phi_{1}+i \phi_{2}\right)
$$

Therefore the potential becomes

$$
V\left(\phi_{1}, \phi_{2}\right)=-\frac{\mu^{2}}{2}\left(\phi_{1}^{2}+\phi_{2}^{2}\right)+\frac{\lambda}{4}\left(\phi_{1}^{2}+\phi_{2}^{2}\right)^{2}
$$

Under a local gauge transformation the fields and vector potentials transform as

$$
\begin{gathered}
\phi(x) \rightarrow \phi^{\prime}(x)=e^{-i \alpha(x)} \phi(x) \\
A_{\mu} \rightarrow A_{\mu}^{\prime}=A_{\mu}-\frac{1}{g} \partial_{\mu} \alpha(x)
\end{gathered}
$$


SSB arises if the vacuum expectation value (VEV) of the scale fields is not invariant under the gauge symmetry. In order to obtain the VEV, we minimize the effective potential 2.53 . If $\mu^{2}>0$ we get the minimum

$$
|\phi|^{2}=\frac{1}{2}\left(\phi_{1}^{2}+\phi_{2}^{2}\right)=\frac{v^{2}}{2} \quad \text { or } \quad \phi_{1}^{2}+\phi_{2}^{2}=v^{2}
$$

Here $v=\sqrt{\frac{\mu^{2}}{\lambda}}$. We can choose $\left\langle\phi_{1}\right\rangle=v,\left\langle\phi_{2}\right\rangle=0$ which are field configurations for classical vacuum. From this we are able to define new quantum fields

$$
\phi_{1}^{\prime}=\phi_{1}-v, \phi_{2}^{\prime}=\phi_{2}
$$

which correspond to oscillations around the minimum so that $\phi^{\prime}=\frac{1}{\sqrt{2}}\left(\phi_{1}^{\prime}+i \phi_{2}^{\prime}\right)$. It is noticed that the condition of $\left\langle\phi_{1}\right\rangle=v$ is necessary for SSB according to the Goldstone's theorem [12]. Hence the new Lagrangian is

$$
\mathcal{L}=\frac{1}{2}\left[\left(D_{\mu} \phi_{1}^{\prime}\right)^{2}+\left(D_{\mu} \phi_{2}^{\prime}\right)^{2}\right]+\frac{\mu^{4}}{4 \lambda}-\mu^{2}{\phi_{1}^{\prime}}^{2}-\lambda v \phi_{1}^{\prime}\left({\phi_{1}^{\prime}}^{2}+{\phi_{2}^{\prime}}^{2}\right)-\frac{\lambda}{4}\left({\phi_{1}^{\prime 2}}^{2}+{\phi_{2}^{\prime}}^{2}\right)^{2}
$$

We can see that there is no ${\phi_{2}^{\prime}}^{2}$ term which implies $\phi_{2}^{\prime}$ is a massless Nambu-Goldstone (N-G) boson. The constant $\mu^{4} / 4 \lambda$ is called "cosmological constant" and can be shifted away due to the absence of gravity. The kinetic term in the Lagrangian can be rewritten in terms of new fields as

$$
\begin{aligned}
\left(D_{\mu} \phi^{\prime}\right)^{\dagger}\left(D_{\mu} \phi^{\prime}\right) & =\left|D_{\mu} \phi^{\prime}\right|^{2}=\left|\left(\partial_{\mu}-i g A_{\mu}\right) \phi^{\prime}\right|^{2} \\
& =\frac{1}{2}\left(\partial_{\mu} \phi_{1}^{\prime}+g A_{\mu} \phi_{2}^{\prime}\right)^{2}+\frac{1}{2}\left(\partial_{\mu} \phi_{2}^{\prime}-g A_{\mu} \phi_{1}^{\prime}\right)^{2} \\
& +\frac{1}{2} g^{2} v^{2} A^{\mu} A_{\mu}-g v A^{\mu}\left(\partial_{\mu} \phi_{2}^{\prime}+g A_{\mu} \phi_{1}^{\prime}\right)
\end{aligned}
$$

Due to SSB, the covariant derivative produces mass term for vector boson with mass $M_{A}=g v$. The main feature of spontaneous breaking of gauge invariance is that gauge vector affiliated with each broken generator gets a longitudinal component and a mass. So far we have already seen how the mass is generated. The additional longitudinal component is given by the N-G boson which gets absorbed by the vector. This can be seen by redefining the fields $\phi$ in polar coordinates as

$$
\begin{aligned}
\phi(x) & =\frac{1}{\sqrt{2}}(v+\eta(x)) e^{i \xi(x) / v} \\
& \simeq \frac{1}{\sqrt{2}}(v+\eta(x)+i \xi(x)+\ldots) \text { for small oscillation }
\end{aligned}
$$


Comparing with Eqs. 2.52$)$ and $(2.56)$ we have

$$
\eta(x) \equiv \phi_{1}^{\prime}, \xi(x) \equiv \phi_{2}^{\prime}
$$

From this point, two configurations associated by a gauge transformation can be made

$$
\begin{aligned}
\phi(x) & \rightarrow \phi^{\prime}(x)=e^{-i \xi(x) / v} \phi(x) \simeq \frac{1}{\sqrt{2}}(v+\eta(x)) \\
A_{\mu} & \rightarrow B_{\mu}=A_{\mu}-\frac{1}{g v} \partial_{\mu} \xi(x)
\end{aligned}
$$

Therefore the Lagrangian transform as

$$
\begin{aligned}
\mathcal{L} \rightarrow \mathcal{L}^{\prime} & =-\frac{1}{4}\left(\partial_{\mu} B_{\nu}-\partial_{\nu} B_{\mu}\right)^{2}+\frac{1}{2}\left|\left(\partial_{\mu}-i g B_{\mu}\right)(v+\eta)\right|^{2}+\frac{\mu^{2}}{2}(v+\eta)^{2}-\frac{\lambda}{4}(v+\eta)^{4} \\
& =-\frac{1}{4}\left(\partial_{\mu} B_{\nu}-\partial_{\nu} B_{\mu}\right)^{2}+\frac{1}{2}\left(\partial_{\mu} \eta\right)^{2}-\frac{1}{2}\left(2 \mu^{2}\right) \eta^{2}+\frac{1}{2} g^{2} v^{2} B^{2}+\left(g^{2} v \eta+\frac{1}{2} g^{2} \eta^{2}\right) B^{2}+\ldots
\end{aligned}
$$

where $B^{2}=B_{\mu} B^{\mu}$. The $\xi(x)$ component disappears in the Lagrangian because it is absorbed as a longitudinal component $B_{\mu}$. What remains is one massive scalar, $\eta$ with mass $m_{\eta}=\sqrt{2} \mu$ and one massive spin-1, $B_{\mu}$ with mass $m_{B}=g v$.

In summary, if a massless gauge boson "eats up" a N-G boson associated with a broken generator, a massive vector meson is produced. This was discovered in the 60s by Peter Higgs 13, 14, Englert and Brout [15, Guralnik, Hagen and Kibble [16 independently and is usually called Higgs mechanism. We shall now see applications of Higgs mechanism in the particular $S U(2)_{L}$ and the SM gauge theories.

\subsubsection{Higgs mechanism for the $S U(2)_{L}$ gauge theory}

In order to break $S U(2)_{L}$ gauge symmetry, one can introduce a complex scaler doublet having 4 degrees of freedom (d.o.f)

$$
\Phi=\left(\begin{array}{l}
\phi_{1} \\
\phi_{2}
\end{array}\right)
$$


The scalar Lagrangian is given by

$$
\mathcal{L}_{S}=-\frac{1}{4} G_{\mu \nu}^{i} G^{i \mu \nu}+\left(D_{\mu} \Phi\right)^{\dagger}\left(D^{\mu} \Phi\right)-V(\Phi), i=1,2,3
$$

where

$$
\begin{aligned}
D_{\mu} \Phi & =\left(\partial_{\mu}-i g \frac{\vec{\tau}}{2} \cdot \vec{W}_{\mu}\right) \Phi \\
G_{\mu \nu}^{i} & =\partial_{\mu} W_{\nu}^{i}-\partial_{\nu} W_{\mu}^{i}+g \epsilon^{i j k} W_{\mu}^{j} W_{\nu}^{k} \\
V(\Phi) & =-\mu^{2}\left(\Phi^{\dagger} \Phi\right)+\lambda\left(\Phi^{\dagger} \Phi\right)^{2}
\end{aligned}
$$

Using the Higgs mechanism recipe, we also find the ground state of the field $\Phi$ to be

$$
\left\langle\Phi^{\dagger} \Phi\right\rangle=\frac{v^{2}}{2} \text { where } v^{2}=\frac{\mu^{2}}{\lambda}\left(\mu^{2}>0 \text { and } \lambda \text { is real }\right) .
$$

By choosing a vacuum alignment

$$
\langle\Phi\rangle=\frac{1}{\sqrt{2}}\left(\begin{array}{c}
0 \\
v
\end{array}\right)
$$

we provide the necessary condition for SSB to happen. Since $v$ is assigned for the VEV of $\operatorname{Re}\left(\phi_{2}\right)$, leaving the other 3 scalar d.o.f of $0-\mathrm{VEV}$ we expect to obtain 1 massive boson and 3 massless N-G bosons which then are eaten up by the vector. To see the spectrum, one would go to "unitary gauge" and write

$$
\Phi(x)=e^{i \vec{\tau} \cdot \frac{\vec{\xi}(x)}{v}}\left(\begin{array}{c}
0 \\
\frac{v+\eta(x)}{2}
\end{array}\right)
$$

It is noticed that the VEVs of $\eta(x)$ and $\xi(x)$ are zero. As seen in the description of Higgs mechanism, $\vec{\xi}$ would give rise to $3 \mathrm{~N}-\mathrm{G}$ bosons and $\eta(x)$ appears to be the Higgs boson. Again, under gauge transformation $U(x) \equiv e^{-i \vec{\tau} \cdot \frac{\vec{\xi}(x)}{v}}$ the term accounted for the additional longitudinal components is

$$
\frac{\vec{\tau}}{2} \cdot \vec{W}_{\mu}^{\prime}=U(x) \frac{\vec{\tau}}{2} \cdot \vec{W}_{\mu} U^{-1}(x)-\frac{i}{g}\left[\partial_{\mu} U(x)\right] U^{-1}(x)
$$

in which N-G bosons are absorbed in the second term. Consequently, the Lagrangian 2.65) transforms in the chosen ground state as

$$
\mathcal{L}_{S}^{\prime}=-\frac{1}{4} G_{\mu \nu}^{i^{\prime}} G^{i^{\prime}, \mu \nu}+\left(D_{\mu}^{\prime} \Phi^{\prime}\right)^{\dagger}\left(D^{\prime \mu} \Phi^{\prime}\right)+\left(\frac{\mu^{2}}{2}(v+\eta(x))^{2}-\frac{\lambda}{4}(v+\eta(x))^{4}\right) .
$$


The mass term for gauge bosons $W_{\mu}^{\prime}$ can be extracted from the second term

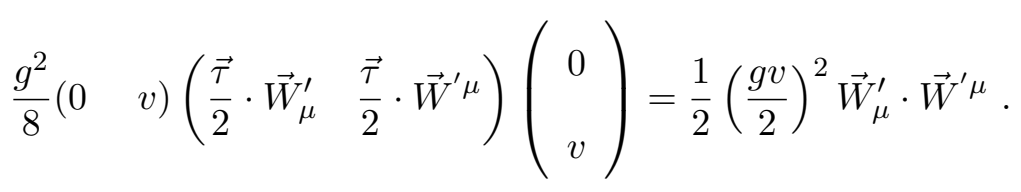

It is worth emphasizing the invariance of the mass term under $S U(2)$ global symmetry. Hence, the gauge bosons $W^{ \pm}, W^{0}$ obtain a mass of $M_{W}=g v / 2$. In addition to masses of gauge bosons, we also generate the mass term of Higgs boson $\eta$ from the potential in Eq. $(2.73): m_{\eta}=\sqrt{2} \mu$.

\subsection{SM gauge theory via Higgs mechanism}

In a theoretical consistent way, we are now ready to write down and discuss the spontaneous breaking of the SM in order to generate masses for vector bosons, the $W^{ \pm}$and the $Z$, and fermions. The fact that the electric charge is conserved and the photon is massless reassures us that the subgroup $S U(3)_{c} \times U(1)_{e m}$ of SM's gauge group remains unbroken under the SSB. Therefore, we will investigate the spontaneous breaking of the subgroup $S U(2)_{L} \times U(1)_{Y}$ of the electroweak interaction in this section.

\subsubsection{Generating masses for gauge bosons}

To break the symmetry spontaneously, we introduce a fermion doublet, similar to the spinor representation of $S U(2)$ in Eq. 2.64, with $T_{L}^{i}$ operators acting on it and it has an eigenvalue $Y / 2$ of $U(1)_{Y}$. The covariant derivative of the gauge $S U(2)_{L} \times U(1)_{Y}$ becomes

$$
D_{\mu}=\partial_{\mu}-i g \vec{T} \cdot \vec{W}_{\mu}-i g^{\prime} \frac{Y}{2} B_{\mu}
$$

where $\vec{W}$ and $B$ are gauge fields of $S U(2)_{L}$ and $U(1)_{Y}$, respectively. We know from our discussion of $S U(2)_{L}$ gauge theory that the scalar field must have 4 d.o.f to give masses to 3 gauge bosons. Thus, it is sufficient to use a complex doublet to generate masses for electroweak gauge bosons. We 
now define a doublet of the form

$$
\Phi=\left(\begin{array}{c}
\phi^{+} \\
\phi^{0}
\end{array}\right) \text { with } \frac{Y}{2}(\Phi)=\frac{1}{2} .
$$

The covariant derivative of $\Phi$ is

$$
D_{\mu} \Phi=\left(\partial_{\mu}-i g \frac{\vec{\tau}}{2} \cdot \vec{W}_{\mu}-\frac{i}{2} g^{\prime} B_{\mu}\right) \Phi
$$

The scalar potential is

$$
V(\Phi)=-\mu^{2}\left(\Phi^{\dagger} \Phi\right)+\lambda\left(\Phi^{\dagger} \Phi\right)^{2}
$$

The VEV of the field $\Phi$ is given by

$$
\langle\Phi\rangle=\left(\begin{array}{c}
0 \\
\frac{v}{\sqrt{2}}
\end{array}\right)
$$

where $v=\sqrt{\mu^{2} / \lambda}$ resulting from minimizing the above potential.

The mass spectrum of gauge bosons can be seen in a chosen "unitary gauge"

$$
\Phi(x)=e^{i \vec{\tau} \cdot \vec{\xi}(x)} v\left(\begin{array}{c}
0 \\
\frac{v+\eta(x)}{2}
\end{array}\right) .
$$

Again, $\langle\eta\rangle=\langle\xi\rangle=0$. Under the transformation $U(\xi(x))$ as in $S U(2)_{L}$ gauge model, we have

$$
\frac{\vec{\tau}}{2} \cdot \vec{W}_{\mu} \rightarrow \frac{\vec{\tau}}{2} \cdot \vec{W}_{\mu}^{\prime}=U(\xi)\left(\frac{\vec{\tau}}{2} \cdot \vec{W}_{\mu}\right) U^{-1}(\xi)-\frac{i}{g}\left[\partial_{\mu} U(\xi)\right] U^{-1}(\xi)
$$

Moreover since the gauge includes $U(1)_{Y}$ group, its gauge field also transforms as

$$
B_{\mu} \rightarrow B_{\mu}^{\prime}=B_{\mu}
$$

Hence the kinetic term in the Lagrangian contains

- The mass term of $S U(2)_{L}$ gauge fields:

$$
\frac{g^{2}}{8}\left(\begin{array}{ll}
0 & v
\end{array}\right)\left(\begin{array}{ll}
\frac{\vec{\tau}}{2} \cdot \vec{W}_{\mu}^{\prime} & \frac{\vec{\tau}}{2} \cdot \vec{W}^{\prime} \mu
\end{array}\right)\left(\begin{array}{c}
0 \\
v
\end{array}\right)=\frac{1}{2}\left(\frac{g v}{2}\right)^{2} \vec{W}_{\mu}^{\prime} \cdot \vec{W}^{\prime \mu}
$$

Note that $\vec{W}_{\mu}^{\prime}=\left(W_{\mu}^{\prime 1}, W_{\mu}^{\prime 2}, W_{\mu}^{\prime 3}\right)$. 
- The mass term of $U(1)_{Y}$ gauge fields:

$$
\frac{g^{\prime 2}}{8}\left(\begin{array}{ll}
0 & v
\end{array}\right)\left(\begin{array}{ll}
B_{\mu}^{\prime} & B^{\prime \mu}
\end{array}\right)\left(\begin{array}{c}
0 \\
v
\end{array}\right)=\frac{1}{2}\left(\frac{g^{\prime} v}{2}\right)^{2} B_{\mu}^{\prime} \cdot B^{\prime \mu}
$$

- The cross terms:

$$
\frac{g g^{\prime}}{2}\left(\begin{array}{ll}
0 & v
\end{array}\right)\left(\frac{\vec{\tau}}{2} \cdot \vec{W}_{\mu}^{\prime}\right) B^{\prime \mu}\left(\begin{array}{c}
0 \\
v
\end{array}\right)=\frac{1}{2}\left(-\frac{g g^{\prime} v^{2}}{2}\right) W_{\mu}^{\prime 3} B^{\prime \mu}
$$

From the above equations, one can put together a mass matrix for $W^{\prime 3}$ and $B^{\prime}$ as

$$
\mathcal{M}=\frac{v^{2}}{4}\left(\begin{array}{cc}
g^{2} & -g g^{\prime} \\
-g g^{\prime} & g^{\prime 2}
\end{array}\right)
$$

Diagonalizing this matrix $\mathcal{M}$ yields two eigenvalues: 0 accounted for the massless photon and $\left(g^{2}+g^{\prime 2}\right) v^{2} / 4$ accounted for the mass of Z-boson. The eigenvectors can be interpreted in terms of a so-called "weak mixing angle" or "Weinberg angle", $\theta_{W}, 17$ as

$$
\begin{aligned}
& Z_{\mu}=\cos \theta_{W} W_{\mu}^{\prime 3}-\sin \theta_{W} B_{\mu}^{\prime} \\
& A_{\mu}=\sin \theta_{W} W_{\mu}^{\prime 3}+\cos \theta_{W} B_{\mu}^{\prime}
\end{aligned}
$$

where

$$
\cos \theta_{W} \equiv \frac{g}{\sqrt{g^{2}+g^{\prime 2}}}, \quad \sin \theta_{W} \equiv \frac{g^{\prime}}{\sqrt{g^{2}+g^{\prime 2}}}
$$

All in all, 4 gauge bosons of the $S U(2)_{L} \times U(1)_{Y}$ gauge theory are

- 2 charged gauge bosons $W_{\mu}^{ \pm}=\left(W_{\mu}^{1} \mp i W_{\mu}^{2}\right) / \sqrt{2}$ with masses $M_{W}=g v / 2$,

- a neutral gauge boson $Z_{\mu}$ with a mass $M_{Z}=v \sqrt{g^{2}+g^{\prime 2}} / 2$,

- a massless photon $A_{\mu}$. 
It is noticed that an important prediction coming out from the investigation of the SM gauge theory is

$$
\frac{M_{W}}{M_{Z}}=\frac{g}{\sqrt{g^{2}+g^{\prime 2}}}=\cos \theta_{W}
$$

From now on it will be more convenient to write all expressions in terms of the above mass eigenstate fields. For example, in a coupling of the vector fields to fermions, the covariant derivative can be written as

$$
\begin{aligned}
D_{\mu}=\gamma_{\mu} \partial_{\mu} & -i \frac{g}{\sqrt{2}}\left(W_{\mu}^{+} T_{L}^{+}+W_{\mu}^{-} T_{L}^{-}\right)-i \frac{1}{\sqrt{g^{2}+g^{\prime 2}}} Z_{\mu}\left(g^{2} T_{L}^{3}-g^{\prime 2} \frac{Y}{2}\right) \\
& -i \frac{g g^{\prime}}{\sqrt{g^{2}+g^{\prime 2}}} A_{\mu}\left(T_{L}^{3}+\frac{Y}{2}\right)
\end{aligned}
$$

where $T_{L}^{ \pm}=\left(T_{L}^{1} \pm i T_{L}^{2}\right)$ and $\vec{T}_{L}=\frac{\vec{\tau}}{2}$ for $S U(2)_{L}$. From this point of view, one may ask if the SM can predict masses of W's and Z bosons in terms of known and measurable physical quantities such as Fermi constant $G_{F}$, angle $\theta_{W}$, etc. We actually can proceed further regarding the identified relation

$$
\frac{G_{F}}{\sqrt{2}}=\frac{g^{2}}{8 m_{W}^{2}}
$$

To estimate the mass of W's boson, we need to know the coupling $g$. This can be done by considering a gauge interaction with the $A_{\mu}$ boson

$$
-i\left(e Q_{L}\right) \bar{\psi}_{L} \gamma^{\mu} \psi_{L} A_{\mu}
$$

Comparing the $A_{\mu}$ interaction in Eqs. 2.91) and 2.93), note that $Q_{L}=T_{L}^{3}+Y / 2$, we get

$$
e=\frac{g g^{\prime}}{\sqrt{g^{2}+g^{\prime 2}}}=g \sin \theta_{W} .
$$

This is another prediction belonging to the SM. Gathering all relations we have been talking about, one can predict the mass of W's bosons

$$
m_{W}^{2}=\frac{\sqrt{2}}{8} \frac{e^{2}}{\sin ^{2} \theta_{W} G_{F}}=\frac{1}{\sqrt{2}} \frac{\pi \alpha}{\sin ^{2} \theta_{W} G_{F}}
$$

where $\alpha \equiv e^{2} /(4 \pi)$, called "electromagnetic coupling constant". We know really well about the coupling constant $\alpha$ and Fermi's constant $G_{F}$ by the time SM was out forward. Hence measuring the angle $\theta_{W}$ will help predict the masses $M_{W}$ and $M_{Z}$. 


\subsubsection{Generating masses for fermions}

Another role played by spontaneous symmetry breaking is to give masses to the fermions. For example, left-handed helicity components of leptons form doublets $l_{L}=\left(\nu_{L}, e_{L}\right)^{T}$ while righthanded one stays as singlet $e_{R}$ under SM gauge theory. Fermions (leptons and quarks) can acquire masses if they have gauge invariant interactions with scalar fields. At the tree level, such Yukawa interactions

$$
\mathcal{L}_{\text {lepton }}=g_{e} \overline{e_{L}} \Phi e_{R}+\text { h.c. with }\langle\Phi\rangle=\left(\begin{array}{c}
0 \\
\frac{v}{\sqrt{2}}
\end{array}\right)
$$

are $S U(2)_{L} \times U(1)_{Y}$ gauge invariant. After the SSB, the mass of electron is given by $m_{e}=g_{e} v / \sqrt{2}$. It is noticed that the value of $v$ is known but that of $g_{e}$ is unconstrained and can be put in by hand. Similarly, we obtain the masses of muon, $m_{\mu}=g_{\mu} v / \sqrt{2}$, and tau, $m_{\tau}=g_{\tau} v / \sqrt{2}$. Again, $g_{\mu}$ and $g_{\tau}$ are free parameters and cannot be predicted by the SM. We will get more discussions on leptons masses in the next chapter.

Quarks likewise acquire their masses from the SSB of the gauge-invariant interactions

$$
\mathcal{L}_{\text {quark }}=g_{d} \bar{q}_{L} \Phi d_{R}+g_{u} \bar{q}_{L} \tilde{\Phi} u_{R}+\text { h.c. }
$$

where $q_{L}=\left(u_{L}, d_{L}\right)^{T}$ and $\tilde{\Phi}=i \tau_{2} \Phi^{*}$. Mass terms have the form of $\left(g_{u} v / \sqrt{2}\right)$ for up-type quarks $(\mathrm{u}, \mathrm{c}, \mathrm{t})$ and $\left(g_{d} v / \sqrt{2}\right)$ for down-type quarks $(\mathrm{d}, \mathrm{s}, \mathrm{b})$. The fact that values of $g_{u}, g_{d}, \ldots$ cannot be measured results in no prediction of quark masses. Additionally, gauge invariance allows extra mass terms such as $l_{e L} \Phi \mu_{R}, l_{e L} \Phi \tau_{R}, l_{\mu L} \Phi e_{R}, \ldots$ Hence, in general we can construct the mass matrix for the up- and down-quark sectors as

$$
\bar{\psi}_{u} \mathcal{M}_{U} \psi_{u}=\frac{v}{\sqrt{2}} \cdot \bar{\psi}_{u}\left(\begin{array}{ccc}
g_{u u} & g_{u c} & g_{u t} \\
g_{c u} & g_{c c} & g_{c t} \\
g_{t u} & g_{t c} & g_{t t}
\end{array}\right) \psi_{u}
$$


and

$$
\bar{\psi}_{d} \mathcal{M}_{D} \psi_{d}=\frac{v}{\sqrt{2}} \cdot \bar{\psi}_{d}\left(\begin{array}{ccc}
g_{d d} & g_{d s} & g_{d b} \\
g_{s d} & g_{s s} & g_{s b} \\
g_{b d} & g_{b s} & g_{b b}
\end{array}\right) \psi_{d} .
$$

Here we define $\psi_{u} \equiv\left(\begin{array}{lll}u & c & t\end{array}\right)^{T}$ and $\psi_{d} \equiv\left(\begin{array}{lll}d & s & b\end{array}\right)^{T}$. The mass matrices $\mathcal{M}_{U}$ and $\mathcal{M}_{D}$ can be complex and non-hermitian. Therefore, we diagonalize each of them using two unitary matrices to obtain eigenvalues as

$$
\begin{aligned}
U_{u, L}^{\dagger} \mathcal{M}_{U} U_{u, R} & =\mathcal{M}_{U, \text { diag }} \\
U_{d, L}^{\dagger} \mathcal{M}_{D} U_{d, R} & =\mathcal{M}_{D, \text { diag }}
\end{aligned}
$$

Taking the $W$ coupling from Eq. (2.24) with the above notations we have

$$
\bar{\psi}_{u, L}^{\prime} U_{u, L}^{\dagger} \gamma_{\mu} U_{d, L} \psi_{d, L}^{\prime}=\bar{\psi}_{u, L}^{\prime} V_{C K M} \gamma_{\mu} \psi_{d, L}^{\prime}
$$

where $\psi_{u, L}^{\prime}=U_{u, L}^{\dagger} \psi_{u, L}, \psi_{d, L}^{\prime}=U_{d, L}^{\dagger} \psi_{d, L}$ and $V_{C K M} \equiv U_{u, L}^{\dagger} U_{d, L}$ which is well-known as the CabibboKobayashi-Masakawa (CKM) matrix. We will go into details of the CKM matrix in the next section because it contains important information on the strength of flavor-changing in the quark sector.

\subsubsection{CKM matrix for quark sector}

The CKM matrix is generalized from the Cabibbo matrix [18] by Kobayashi and Masakawa in 1973 [19,20]. It describes the mixings between the three quark families in the SM and therefore, has the form of a $3 \times 3$ matrix

$$
V_{C K M}=\left(\begin{array}{ccc}
V_{d d} & V_{d s} & V_{d b} \\
V_{s d} & V_{s s} & V_{s b} \\
V_{b d} & V_{b s} & V_{b b}
\end{array}\right)
$$


In general, the matrix $V_{C K M}$ can have complex elements and is a unitary matrix with 9 parameters. They are 3 rotational angles called Cabibbo angles [18 and 6 phases. However, 5 phases can be removed by making phases rotations of quark fields as

$$
q_{L}^{k} \rightarrow e^{i \alpha_{k}} q_{L}^{k}
$$

Therefore, $V_{C K M}$ for 3 families has 3 mixing angles and 1 phase. One can parametrize $V_{C K M}$ in many different ways but we found such a standard parametrization [21] as follows would be sufficient.

$$
\begin{aligned}
& V_{C K M}=\left(\begin{array}{ccc}
1 & 0 & 0 \\
0 & c_{23} & s_{23} \\
0 & -s_{23} & c_{23}
\end{array}\right)\left(\begin{array}{ccc}
c_{13} & 0 & s_{13} e^{-i \delta} \\
0 & 1 & 0 \\
-s_{13} e^{i \delta} & 0 & c_{13}
\end{array}\right)\left(\begin{array}{ccc}
c_{12} & s_{12} & 0 \\
-s_{12} & c_{12} & 0 \\
0 & 0 & 1
\end{array}\right) \\
& =\left(\begin{array}{ccc}
c_{12} c_{13} & s_{12} c_{13} & s_{13} e^{-i \delta} \\
-s_{12} c_{23}-c_{12} s_{23} s_{13} e^{i \delta} & c_{12} c_{23}-s_{12} s_{23} s_{13} e^{i \delta} & s_{23} c_{13} \\
s_{12} s_{23}-c_{12} c_{23} s_{13} e^{i \delta} & -c_{12} s_{23}-s_{12} c_{23} s_{13} e^{i \delta} & c_{23} c_{13}
\end{array}\right),
\end{aligned}
$$

where $s_{i j}=\sin \theta_{i j}, c_{i j}=\cos \theta_{i j}, \theta_{i j} \in[0, \pi / 2]$ and $\delta$ is the phase responsible for all $C P$-violating phenomena in flavor-changing processes in the SM. Because of such a hierarchy in angles, i.e. $s_{12} \gg s_{23} \gg s_{13}$, one can set $\lambda \equiv s_{12}$ and use the Wolfenstein parametrization 21,22

$$
V_{C K M, W}=\left(\begin{array}{ccc}
1-\lambda^{2} / 2 & \lambda & A \lambda^{3}(\rho-i \eta) \\
-\lambda & 1-\lambda^{2} / 2 & A \lambda^{2} \\
A \lambda^{3}(1-\rho-i \eta) & -A \lambda^{2} & 1
\end{array}\right),
$$

in which $A, \rho, \eta$ are real parameters of $\mathcal{O}(1)$. The CKM matrix elements play an important role in the SM, so it is important to determine them precisely. Let us summarize the current status of the CKM matrix [21].

(1) $\left|V_{u d}\right| \simeq 1-\lambda^{2} / 2=0.97417 \pm 0.00021$ from superallowed $0^{+} \rightarrow 0^{+}$nuclear beta decays or using pion decay by shooting a proton beam into a target. 
(2) $\left|V_{u s}\right| \simeq \lambda=0.2248 \pm 0.0006$ from kaon decays and the ratio of the kaon and pion decay constants.

(3) $\left|V_{c d}\right|=0.220 \pm 0.005$ from semileptonic charm decays, leptonic decays and neutrino scattering data.

(4) $\left|V_{c s}\right|=0.995 \pm 0.016$ from semileptonic $D$ or leptonic $D_{s}$ decays.

(5) $\left|V_{c b}\right|=(40.5 \pm 1.5) \times 10^{-3}$ from exclusive and inclusive semileptonic decays of $B$ mesons to charm.

(6) $\left|V_{u b}\right|=(4.09 \pm 0.39) \times 10^{-3}$ from $B \rightarrow X_{u} l \bar{\nu}$ decays. However there are many theoretical uncertainties in hadronic matrix elements.

(7) $\left|V_{t d}\right|=(8.2 \pm 0.6) \times 10^{-3}, \quad\left|V_{u b}\right|=(40.0 \pm 2.7) \times 10^{-3}$

from $B-\bar{B}$ oscillations at loop-levels which are either box diagrams with top quarks or loop-mediated rare $K$ and $B$ decays.

(8) $\left|V_{t b}\right|=1.009 \pm 0.031$ from top decays $t \rightarrow W b$ and $t \rightarrow W q$ of the CDF and D $\varnothing$ experiments.

Using the prescription of Refs. 23 25] gives

$$
\begin{array}{ll}
\lambda=0.22496 \pm 0.00048, & A=0.823 \pm 0.013, \\
\bar{\rho}=0.141 \pm 0.019, & \bar{\eta}=0.349 \pm 0.012 .
\end{array}
$$

The fit results in the numerical form of the CKM matrix

$$
V_{C K M}=\left(\begin{array}{rrr}
0.97434_{-0.00012}^{+0.00011} & 0.22506 \pm 0.00050 & 0.00357 \pm 0.00015 \\
0.22492 \pm 0.00050 & 0.97351 \pm 0.00013 & 0.0411 \pm 0.0013 \\
0.00875_{-0.00033}^{+0.00032} & 0.0403 \pm 0.0013 & 0.99915 \pm 0.00005
\end{array}\right)
$$

Measuring the phase of the CKM matrix is crucial in the study of the $C P$ violation. However we will not go into the details of the measurement as the $C P$ phase is not the focus of this dissertation. 
In the next chapter we will discuss the details of a lepton mixing matrix that is analogous to the CKM matrix of quark sector.

\subsection{Standard Model: Limitations and Challenges}

Before closing this chapter let us summarize what we have learned so far about the SM. This "renormalizable" theory has been proven in describing the three fundamental forces: electromagnetic, weak and strong forces which form the gauge group $S U(3)_{C} \times S U(2)_{L} \times U(1)_{Y}$. Under such a local symmetry, the Higgs mechanism arose to generate fermion and gauge boson masses. SM has governed many experimental designs and been thoroughly tested at various experiments.

Even though the SM was very successful theory to describe the fundamental particles and interactions, it is facing some challenges and limitations. Several of them are highlighted as follows.

- First of all, the SM does predict the existence of fermions and gauge bosons but cannot adequately explain their observed masses as well as the mass hierarchy. Therefore one needs inputs from experiments and some additional theories.

- In the SM, neutrinos have exactly zero mass. Neutrinos' features include three neutrinos belonging to three lepton families, conserved lepton number, distinguishable neutrinos and antineutrinos in the sense that all neutrinos are left-handed and all antineutrinos are righthanded. However, experimental data from neutrino oscillations have shown us that at least two of three neutrinos have tiny but non-zero masses. Moreover, the origin of neutrino masses remains mysterious.

- SM cannot predict flavor violating processes such as $\mu \rightarrow e \gamma$ or $\mu-e$ conversion.

- SM cannot answer questions related to dark matter and dark energy constituting $95.1 \%$ of total mass - energy content of the Universe: What are they? What is the cause of the 
observed accelerated expansion of the Universe? etc.

Regarding the above challenges, we now have an urge for taking further steps into physics Beyond the Standard Model. Next chapter will provide us details on evidences for the new physics which are neutrino "oscillations", masses and mixings. They will serve as a base for our research work presented in the following chapters. 


\section{Chapter 3}

\section{Neutrinos}

Neutrinos are subatomic particles produced by the decay of radioactive elements and are elementary particles. The name "neutrino" meaning "little neutron" in Italian tells us that a neutrino carries no charge and therefore, only undergoes weak force and gravity. Neutrinos are also very elusive due to the fact that they hardly interact with matters when traveling across the Universe. There are some interesting facts about neutrinos that people may not know. Every second about 65 billion neutrinos pass through just $1 \mathrm{~cm}^{2}$ of area on the Earth. Surprisingly, we feel nothing about such a huge amount of neutrinos traversing our body. In order to stop a beam of neutrinos, one will need a wall of lead of 1.6 light years thickness. Until now, there is about 330

neutrinos $/ \mathrm{cm}^{3}$ left over from the Big Bang which happened billions years ago. These are just a few descriptions of the neutrino and we will learn more about this tiny creature in the following sections.

\subsection{Brief history of neutrinos}

It started with the $\beta$-decay: $A_{Z} \rightarrow A_{Z+1}+e^{-}$in which electron's energy is not equal to mass difference between $A_{Z}$ and $A_{Z+1}$. In order to preserve energy-momentum in the $\beta$-decay, Wolfgang Pauli proposed the existence of a light neutral particle of spin $1 / 2$ in $\beta$-decay, called it "neutron" in 1930. However 2 years later James Chadwick discovered the real neutron: the proton-like neutral 
hadron so this made Pauli's particle become unnamed for a while. In 1934, Enrico Fermi renamed Pauli's particle the "neutrino". He published a successful theory which established the existence of the neutrino but the particle itself remained elusive which might never be detected. From this moment neutrinos come into real life and promote several experiments in a discovery of these tiny particles.

In 1956, the electron neutrino, $\nu_{e}$, had finally been observed. It came from the work of two scientists Fred Reines and Clyde Cowan 26, 27] on designing a detector which incorporated Cadmium (Cd) and was located underground in Savannah River, South Carolina. They used the inverse beta decay

$$
\bar{\nu}_{e}+p \rightarrow e^{+}+n
$$

This reaction is followed by a positron-electron annihilation to create two gamma rays. On the other hand the neutron is captured by a $\mathrm{Cd}$ nucleus, producing another gamma ray when the excited nucleus releases energy. In 1962, the second type of neutrino called muon neutrino, $\nu_{\mu}$, was discovered in an accelerator at Brookhaven by Leon Lederman, Mel Schwartz and Jack Steinberger 28. It led to a Nobel prize in 1988. In 1975, with the discovery of the third charged lepton, $\tau$, by Marty Perl 29] (Nobel Prize 1995) many physicists postulated the idea of having a third neutrino called $\nu_{\tau}$ which was finally observed after 25 years in 2000 at Fermilab 30, 31.

\subsection{Neutrino Oscillations}

In this section, I will briefly show you evidences of neutrino oscillations which have resulted in the 2015 Nobel Prize in Physics [33] for Takaaki Kajita and Arthur B. McDonald. The discovery of these oscillations shows that neutrinos have mass and the neutrino sector, therefore, has had to be completely reconsidered. Two different causalities were interpreted: Solar Neutrino Problem and Atmospheric Neutrino Anomaly. 
Total Rates: Standard Model vs. Experiment

Bahcall-Pinsonneault 2000

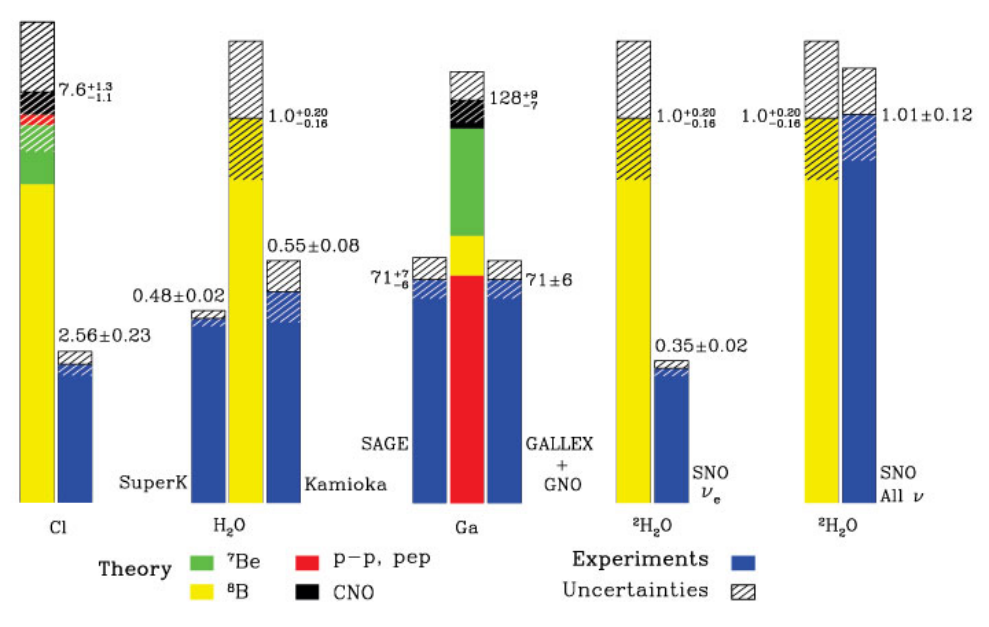

Figure 3.1: Solar neutrino observations

The solar neutrino problem started with Ray Davis's Chlorine 37 solar neutrino experiment [34,35] in which the electron neutrino flux from the Sun was only about one third of that expected from the predictions of the standard solar model (SSM). In late 1980s, Kamiokande-II [36] observed $46( \pm 15) \%$ of the expected flux of greater than $9.3 \mathrm{MeV}$ high energy solar neutrino. A few years later, the GALLEX and SAGE 37$]$ saw about $62( \pm 10) \%$ of SSM prediction for energies greater than $0.233 \mathrm{MeV}$. If we believe in experiment results, the candidate explanations for these observations could be either there is something wrong with the solar model or there is something wrong with the neutrinos. This is the first hint of neutrino oscillations.

Meanwhile, a so-called atmospheric neutrino anomaly [38,39] was raised by measuring atmospheric neutrinos produced by cosmic rays striking nuclei in the Earth atmosphere. As a result, hadronic showers typically containing $\pi$ mesons are produced. These mesons then decay to other 
lighter and more stable particles, for example,

$$
\begin{aligned}
& \pi^{+} \rightarrow \mu^{+}+\nu_{\mu} \\
& \mu^{+} \rightarrow e^{+}+\nu_{e}+\overline{\nu_{\mu}} .
\end{aligned}
$$

The ratio of number of muon neutrinos to that of electron neutrinos that we expect to see at the detector is 2-to-1. However, we observed this ratio to be 1.3-to-1 at the Super-Kamiokande in 1998 [40]. Since the observed ratio was deviated from the predicted one, it was a good indicator for neutrino oscillations. The results of the Super-Kamiokande were marked as "the discovery of neutrino oscillations".

In order to understand how one type of neutrinos can change into another one, we describe quantum mechanically the oscillation of one quantum state into another. Although neutrino is in a well-defined flavor state when it is produced and when it interacts, it is not in a well-defined flavor state when it travels between the two. In fact, one flavor state of neutrinos is a mixture of three mass states with definite masses. Therefore, neutrino oscillations arise from a mixture between the flavor and mass eigenstates of neutrinos.

$$
\left|\nu_{\alpha}\right\rangle=\sum_{i} U_{\alpha i}^{l e p t o n}\left|\nu_{i}\right\rangle
$$

where $\left|\nu_{\alpha}\right\rangle$ is flavor eigenstate. $\alpha=e, \mu, \tau,\left|\nu_{i}\right\rangle$ is mass eigenstate. $\mathrm{i}=1,2,3$, and $U_{\alpha i}^{\text {lepton }}$ is the lepton mixing matrix or the well-known Pontecorvo-Maki-Nakagawa-Sakata matrix which will be discussed later in section 3.3. Assume that a neutrino is born with flavor $\alpha$ at $t=0$ so Eq. (3.4) can be rewritten as

$$
\left|\nu_{\alpha}(0)\right\rangle=\sum_{i} U_{\alpha i}^{l e p t o n}\left|\nu_{i}(0)\right\rangle
$$

In the rest frame of $\nu_{i}$ and at a later time $t=\tau_{i}$ we have

$$
\left|\nu_{i}\left(\tau_{i}\right)\right\rangle=e^{-i m_{i} \tau_{i}}\left\langle\nu_{i}(0)\right\rangle
$$

On the other hand, if one considers the process in the lab frame then

$$
e^{-i m_{i} \tau_{i}}=e^{-i\left(E_{i} t-p_{i} L\right)} \simeq e^{-i\left(E_{i}-p_{i}\right) L},
$$


where $t \simeq L$ for the case of extremely relativistic neutrinos as we are investigating. It is noticed that $\nu_{\alpha}$ produced with the definite 3 -momentum $\vec{p}$ implies

$$
E_{i} \simeq p_{i}+\frac{m_{i}^{2}}{2 p_{i}}
$$

Hence

$$
e^{-i m_{i} \tau_{i}} \simeq e^{-i\left(m_{i}{ }^{2} / 2 p\right) L} \simeq e^{-i\left(m_{i}{ }^{2} / 2 E\right) L}
$$

After traveling a distance $L$ Eq. (3.5) tells us

$$
\left|\nu_{\alpha}(L)\right\rangle \simeq \sum_{i} U_{\alpha i}^{l e p t o n} e^{-i\left(m_{i}^{2} / 2 E\right) L}\left|\nu_{i}\right\rangle
$$

To clearly see how $\nu_{\alpha}$ transforms into another flavor neutrino $\nu_{\beta}$ we write the mass eigenstate $\nu_{i}$ in terms of the flavor eigenstate $\nu_{\beta}$ as

$$
\left|\nu_{i}\right\rangle=\sum_{\beta} U_{\beta i}^{\text {lepton* }}\left|\nu_{\beta}\right\rangle
$$

Eq. (3.10) now becomes

$$
\left|\nu_{\alpha}(L)\right\rangle \simeq \sum_{\beta} \sum_{i}\left(U_{\alpha i}^{l} e^{-i\left(m_{i}^{2} / 2 E\right) L} U_{\beta i}^{l}\right)\left|\nu_{\beta}\right\rangle .
$$

where 'l' stands for 'lepton'. The probability for a neutrino born with flavor $\alpha$ to "transform" into another neutrino with flavor $\beta$ after traveling a distance $L$ is given by

$$
\begin{aligned}
P_{\nu_{\alpha} \rightarrow \nu_{\beta}} & =\left|\left\langle\nu_{\beta} \mid \nu_{\alpha}(L)\right\rangle\right|^{2} \\
& =\sum i, j U_{\alpha i} U_{\beta i}^{*} U_{\alpha j}^{*} U_{\beta j} e^{-i \frac{m_{i}^{2}-m_{j}^{2}}{2 E} L}
\end{aligned}
$$

Let us examine an example for 2-flavor neutrino oscillation in which flavor eigenstates are $\nu_{e}, \nu_{\mu}$ and mass eigenstates are $\nu_{1}, \nu_{2}$ having masses of $m_{1}, m_{2}$.

$$
\begin{aligned}
& \left|\nu_{e}\right\rangle=\cos \theta\left|\nu_{1}\right\rangle+\sin \theta\left|\nu_{2}\right\rangle \\
& \left|\nu_{\mu}\right\rangle=-\sin \theta\left|\nu_{1}\right\rangle+\cos \theta\left|\nu_{2}\right\rangle
\end{aligned}
$$

For a neutrino, e.g., $\nu_{e}$ carrying an energy, $E$, by the time it reaches the detector which is a distance $L$ away from the source its quantum state becomes

$$
\left|\nu_{e}(L)\right\rangle=\cos \theta e^{-i m_{1}^{2} L / 2 E}\left|\nu_{1}\right\rangle+\sin \theta e^{-i m_{2}^{2} L / 2 E}\left|\nu_{2}\right\rangle
$$


By writing the mass eigenstates $\nu_{1}, \nu_{2}$ in terms of the flavor $\nu_{\mu}$

$$
\begin{aligned}
& \left|\nu_{1}\right\rangle=\cos \theta\left|\nu_{e}\right\rangle-\sin \theta\left|\nu_{\mu}\right\rangle, \\
& \left|\nu_{2}\right\rangle=\sin \theta\left|\nu_{e}\right\rangle+\cos \theta\left|\nu_{\mu}\right\rangle,
\end{aligned}
$$

the probability for a neutrino born with flavor $\nu_{e}$ to change into one with flavor $\nu_{\mu}$ after traveling a distance $\mathrm{L}$ is expressed as

$$
\begin{aligned}
P\left(\nu_{e} \rightarrow \nu_{\mu}\right) & =\left|\left\langle\nu_{\mu} \mid \nu_{e}(L)\right\rangle\right|^{2} \\
& =\frac{1}{2} \sin ^{2} 2 \theta\left(1-\cos \frac{\Delta m_{21}^{2} L}{2 E}\right)=\sin ^{2} 2 \theta \cdot \sin ^{2}\left(\frac{\Delta m_{21}^{2} L}{4 E}\right)
\end{aligned}
$$

where $\Delta m_{21}^{2}=m_{2}^{2}-m_{1}^{2}$. It is noticed that there are two unknowns: the angle $\theta$ and the masssquared difference $\Delta m_{21}^{2}$ that present neutrino oscillation phenomenon. Therefore, if we can measure the neutrino oscillation probability as a function of the neutrino's traveling distance L or the neutrino's energy E, it is possible to extract information on neutrino masses and mixings. It is required in Eq. 3.19 that the minimum value of a generic mass-squared difference $\Delta m^{2}$ is about $2 E / L$. Therefore, for experiments searching for neutrino oscillations, this requirement is served as the sensitivity of the experiments. Table (3.1) shows details on several different neutrino oscillations experiments 41].

Table 3.1: Sensitivity of different neutrino oscillation experiments

\begin{tabular}{||l|c|c|c|c|c||}
\hline Source & Experiments & Type of $\nu$ & $\bar{E}(\mathrm{MeV})$ & $L(k m)$ & $\min \left(\Delta m^{2}\right)$ \\
\hline \hline Reactor & $\begin{array}{c}\text { Chooz, Daya Bay, } \\
\text { RENO, Double Chooz }\end{array}$ & $\bar{\nu}_{e}$ & $\sim 1$ & 1 & $\sim 10^{-3}$ \\
\hline Reactor & KamLAND & $\bar{\nu}_{e}$ & $\sim 1$ & 100 & $\sim 10^{-5}$ \\
\hline Accelerator & past & $\nu_{\mu}, \bar{\nu}_{\mu}$ & $\sim 10^{3}$ & 1 & $\sim 1$ \\
\hline Accelerator & $\begin{array}{c}\text { K2K, MINOS, T2K, } \\
\text { OPERA, NO } \nu \mathrm{A}\end{array}$ & $\nu_{\mu}, \bar{\nu}_{\mu}$ & $\sim 10^{3}$ & 1000 & $\sim 10^{-3}$ \\
\hline Atmospheric $\nu$ 's & Super-K, MINOS, IceCube & $\nu_{\mu, e}, \bar{\nu}_{\mu, e}$ & $\sim 10^{3}$ & $10^{4}$ & $\sim 10^{-4}$ \\
\hline Sun & & $\nu_{e}$ & $\sim 1$ & $1.5 \times 10^{8}$ & $\sim 10^{-11}$ \\
\hline
\end{tabular}




\subsection{Neutrino masses and mixings}

As discussed in section 3.2 , if neutrinos have mass and therefore are able to change their flavors, both of the atmospheric and solar neutrino anomalies could be revealed. This is because muon neutrinos from the atmosphere which might oscillate into tau neutrinos would be experimentally undetectable. Similarly, if electron neutrinos from the Sun can change into muon or tau neutrinos, they will interact at a significantly lower rate so one cannot detect them.

Recall the Eq. (3.4) in the previous section, we will now discuss on how to achieve the lepton mixing matrix. Let us consider the following charged current interaction between leptons and the $W^{+}$boson

$$
\mathcal{L}_{C C}=g_{l} \bar{\nu}_{L}^{0} \gamma_{\mu} L_{L}^{0} W^{\mu+}+\text { h.c. }
$$

where $\nu_{L}^{0}=\left(\nu_{e L}^{0}, \nu_{\mu L}^{0}, \nu_{\tau L}^{0}\right)^{T}$ and $L_{L}^{0}=\left(e_{L}^{0}, \mu_{L}^{0}, \tau_{L}^{0}\right)^{T}$ are gauge eigenstates whereas $\nu_{L}=$ $\left(\nu_{1 L}^{0}, \nu_{2 L}^{0}, \nu_{3 L}^{0}\right)^{T}$ and $L_{L}=\left(e_{L}, \mu_{L}, \tau_{L}\right)^{T}$ are mass eigenstates. A relation between gauge and mass eigenstates is established as

$$
L_{L}^{0}=U_{l_{L}} L_{L}, \quad \nu_{L}^{0}=U_{\nu_{L}} \nu_{L}
$$

Therefore, the charged current interaction can be written as

$$
\begin{aligned}
\mathcal{L}_{c} & =g_{l} \bar{\nu}_{L} \gamma_{\mu} U_{\nu_{L}}^{-1} U_{l_{L}} L_{L} W^{\mu+}+\text { h.c. } \\
& =g_{l} \bar{\nu}_{L} \gamma_{\mu} U^{\text {lepton }} L_{L} W^{\mu+}
\end{aligned}
$$

where $U^{\text {lepton }}=U_{\nu_{L}}^{-1} U_{l_{L}}$ is called the lepton mixing matrix. It is noticed that $U^{\text {lepton }}$ is a unitary matrix, just like $V_{C K M}$. Generally, matrix elements of $U^{\text {lepton }}$ are expected to be complex.

Assuming that there are 3-flavor neutrino mixing in vacuum, one can describe all enthralling neutrino oscillation data. The decay width of the Z-boson data tells us that only 3 light flavor neutrinos coupled to $\mathrm{Z}$ [42]. If there exists massive neutrinos their masses must be above $M_{Z} / 2$ in order to avoid the $Z$ from decaying into $2 \nu$ 's. The currently published data from Planck 43 
has shown that at least 3 of the neutrinos, denoted as $\nu_{1}, \nu_{2}, \nu_{3}$ in Eq. (3.4), must be light with masses $\lesssim 0.23 \mathrm{eV}$ and must have different real and positive masses, $m_{1} \neq m_{2} \neq m_{3}$. Within the framework of 3-neutrino oscillations, the neutrino flavor eigenstates $\nu_{e}, \nu_{\mu}$, and $\nu_{\tau}$ are related to the neutrino mass eigenstates $\nu_{1}, \nu_{2}$, and $\nu_{3}$ by the $U^{\text {lepton }}$ as

$$
\left(\begin{array}{c}
\nu_{e} \\
\nu_{\mu} \\
\nu_{\tau}
\end{array}\right)=\left(\begin{array}{ccc}
U_{e 1} & U_{e 2} & U_{e 3} \\
U_{\mu 1} & U_{\mu 2} & U_{\mu 3} \\
U_{\tau 1} & U_{\tau 2} & U_{\tau 3}
\end{array}\right)\left(\begin{array}{c}
\nu_{1} \\
\nu_{2} \\
\nu_{3}
\end{array}\right)
$$

This matrix $U^{\text {lepton }}$ was first introduced in 1962 by Z. Maki, M. Nakagawa and S. Sakata 44 to support the idea of neutrino oscillations predicted by B. Pontecorvo in 1967 [45, 46]. It was then named after the founders as PMNS (Pontecorvo-Maki-Nakagawa-Sakata) matrix. Henceforth we will use a notation $U_{P M N S}$ to refer to the matrix.

In general, if there are $n$ flavor neutrinos and $n$ massive neutrinos then the $U_{P M N S}$ is a $n \times n$ complex matrix having $2 n^{2}$ parameters. Since $U_{P M N S}$ is unitary the number of parameters is reduced to $n^{2}$ which is composed of $n(n-1) / 2$ Euler angles and $n(n+1) / 2$ phases. If neutrinos are so-called Dirac particles, i.e. particles and anti-particles are distinct, one can absorb $2 n-1$ phases leaving $(n-1)(n-2) / 2$ independent phases. For $n=3, U_{P M N S}$ can be parameterized by 3 Euler angles and 1 "Dirac phase" as follows

$$
U_{P M N S}=V=\left(\begin{array}{ccc}
c_{12} c_{13} & s_{12} c_{13} & s_{13} e^{-i \delta} \\
-s_{12} c_{23}-c_{12} s_{23} s_{13} e^{i \delta} & c_{12} c_{23}-s_{12} s_{23} s_{13} e^{i \delta} & s_{23} c_{13} \\
s_{12} s_{23}-c_{12} c_{23} s_{13} e^{i \delta} & -c_{12} s_{23}-s_{12} c_{23} s_{13} e^{i \delta} & c_{23} c_{13}
\end{array}\right)
$$

where $s_{i j} \equiv \sin \left(\theta_{i j}\right), c_{i j} \equiv \cos \left(\theta_{i j}\right), \theta_{i j}=\left[0, \frac{\pi}{2}\right], \delta=[0,2 \pi]$ is the Dirac phase. However if neutrinos have so-called Majorana nature, i.e. neutrinos are their own anti-particles, then $U_{P M N S}$ contains $n(n+1) / 2$ phases because massive Majorana neutrino fields cannot "absorb" phases as opposed to Dirac fields. In the case of $n=3$ we now have additional 2 "Majorana phases" and $U_{P M N S}$ can be cast in the product

$$
U_{P M N S}=V \cdot P
$$


where

$$
P=\left(\begin{array}{ccc}
1 & 0 & 0 \\
0 & e^{i \frac{\alpha_{21}}{2}} & 0 \\
0 & 0 & e^{i \frac{\alpha_{31}}{2}}
\end{array}\right)
$$

and $\alpha_{21}, \alpha_{31}$ are Majorana phases. We will get to further details on Dirac and Majorana neutrinos in the next section. The three $\theta_{i j}$ angles are measured mainly with three types of experiments: atmospheric neutrino mixing, solar neutrino mixing and reactor neutrino mixing.

Let us go back to the Super-Kamiokande experiment in 1998 [40] that raised the atmospheric neutrino anomaly. Due to the fact that the observation of muon neutrinos yielded a significantly smaller number than expected, the standard interpretation is that muon are oscillating into tau neutrinos. The data can be well described by approximately maximal atmospheric mixing $\left|U_{\mu 3}\right| \approx$ $\left|U_{\tau 3}\right| \approx 1 / \sqrt{2}$. This corresponds to

$$
\sin \theta_{23} \approx 1 / \sqrt{2} \quad \text { or } \quad \theta_{23} \approx 45^{\circ}
$$

The angle $\theta_{23}$ is also known as the atmospheric mixing angle. Moreover, we can identify the heavy atmospheric neutrino of mass $m_{3}$ as being approximately

$$
\nu_{3} \approx \frac{\nu_{\mu}+\nu_{\tau}}{\sqrt{2}}
$$

Other experiments such as MINOS [47,48] and IceCube also study atmospheric neutrinos and their data are included in the global fit which will be listed in Table 3.2 .

Solar neutrino mixing experiments involve measurements of the angle $\theta_{12}$ or "solar mixing angle". The standard interpretation is that the electron neutrinos $\nu_{e}$ disappear on the way to detectors on Earth because they oscillates into muon or tau neutrinos. The trimaximal solar mixing $\left|U_{e 2}\right| \approx\left|U_{\mu 2}\right| \approx\left|U_{\tau 2}\right| \approx 1 / \sqrt{3}$ implies that

$$
\sin \theta_{12} \approx 1 / \sqrt{3}
$$


or $\theta_{12} \approx 35^{\circ}$. In consistent with the mixing, a solar neutrino of mass $m_{2}$ was given approximately by

$$
\nu_{2} \approx \frac{\nu_{e}+\nu_{\mu}-\nu_{\tau}}{\sqrt{3}}
$$

SNO [49] and KamLAND [50] were the other two main experiments studying solar neutrinos.

The last neutrino mixing angle $\theta_{13}$ was established to be non-zero by reactor experiments Double Chooz [51], Daya Bay [52], and RENO [53]. The measured value of $\theta_{13}$ was sufficiently large so it widens the door to look into and measure the unknown CP violating phase $\delta$ in the $U_{P M N S}$ and neutrino masses. The latest results were well-reported:

$$
\begin{aligned}
\text { Double Chooz: } & \sin ^{2} 2 \theta_{13}=0.090_{-0.029}^{+0.032}, \\
\text { Daya Bay: } & \sin ^{2} 2 \theta_{13}=0.084 \pm 0.005, \\
\text { RENO: } & \sin ^{2} 2 \theta_{13}=0.082 \pm 0.009 \pm 0.006 .
\end{aligned}
$$

This corresponds to

$$
\left|U_{e 3}\right|=\sin \theta_{13} \approx 0.15
$$

or a reactor angle $\theta_{13} \approx 8.5^{\circ}$.

Because the oscillation of neutrinos can only tell us about the mass-squared difference so the order and the absolute values of $m_{1}, m_{2}$, and $m_{3}$ on the mass scale remain unknown. This brings up the well-known neutrino mass hierarchy problem or to be more friendly, neutrino mass ordering. In the case of 3-neutrino mixing there are two mass-squared difference which are $\Delta m_{21}^{2} \neq 0$ and $\Delta m_{31}^{2} \neq 0$. As a convention we assume that $m_{1}<m_{2}$, so that $\Delta m_{21}^{2}>0$. Therefore it breaks down to two different scenarios:

- Normal Hierarchy: $m_{1}<m_{2}<m_{3}$

- Inverted Hierarchy: $m_{3}<m_{1}<m_{2}$ 
At this point, Fig. 3.2 will provide a brief summary of neutrino mass hierarchy in combination with the information about neutrino masses $m_{1}, m_{2}$, and $m_{3}$ resulting from neutrino mixing experiments. The recent global fit values of neutrino oscillation parameters are given in Table (3.2).

Table 3.2: Mixing parameters from global three-neutrino oscillation data taken from [54,55]. The definition of $\Delta m^{2}$ used is: $\Delta m^{2}=m_{3}^{2}-\left(m_{2}^{2}+m_{1}^{1}\right) / 2$. Thus, $\Delta m^{2}=\Delta m_{31}^{2}-\Delta m_{21}^{2} / 2>0$, if $m_{1}<m_{2}<m_{3}$ (normal hierarchy), and $\Delta m^{2}=\Delta m_{32}^{2}+\Delta m_{21}^{2} / 2<0$ for $m_{3}<m_{1}<m_{2}$ (inverted hierarchy).

\begin{tabular}{|c||c|c|}
\hline Mixing Parameters & Normal Hierarchy & Inverted Hierarchy \\
\hline \hline $\sin ^{2} \theta_{12}$ & $0.308 \pm 0.017$ & $0.308 \pm 0.017$ \\
$\sin ^{2} \theta_{23}$ & $0.437_{-0.023}^{+0.033}$ & $0.455_{-0.031}^{+0.139}$ \\
$\sin ^{2} \theta_{13}$ & $0.0234_{-0.0019}^{+0.0020}$ & $0.024_{-0.0022}^{+0.0019}$ \\
$\delta / \pi$ & $1.39_{-0.27}^{+0.38}$ & $1.31_{-0.33}^{+0.29}$ \\
$\Delta m_{21}^{2}=m_{2}^{2}-m_{1}^{2}$ & $\left(7.54_{-0.22}^{+0.26}\right) \times 10^{-5} \mathrm{eV}^{2}$ & $\left(7.54_{-0.22}^{+0.26}\right) \times 10^{-5} \mathrm{eV}^{2}$ \\
$\Delta m^{2}=\left|m_{3}^{2}-\left(m_{1}^{2}+m_{2}^{2}\right) / 2\right|$ & $(2.43 \pm 0.06) \times 10^{-3} \mathrm{eV}^{2}$ & $(2.38 \pm 0.06) \times 10^{-3} \mathrm{eV}^{2}$ \\
\hline
\end{tabular}

Despite the fact that we cannot know exactly the absolute neutrino masses we have enough information to constrain the neutrino mass. Cosmological results tell us that the sum of all neutrino masses has to be less than $0.23 \mathrm{eV}$ [43]. In addition, from neutrino oscillation experiments we learn that the largest mass-squared difference is $\Delta m_{\text {atm }}^{2} \approx 2.4 \times 10^{-3} \mathrm{eV}^{2}$ 41. One implies that the heaviest neutrinos will have mass $m_{\nu} \gtrsim 4.9 \times 10^{-2} \mathrm{eV}$. Therefore, if we combine the results from cosmology and neutrino oscillation experiments one can set a constraint on the mass of a heaviest neutrino to be

$$
4.9 \times 10^{-2} \mathrm{eV} \lesssim m_{\nu}^{\text {heaviest }} \lesssim 0.23 \mathrm{eV}
$$




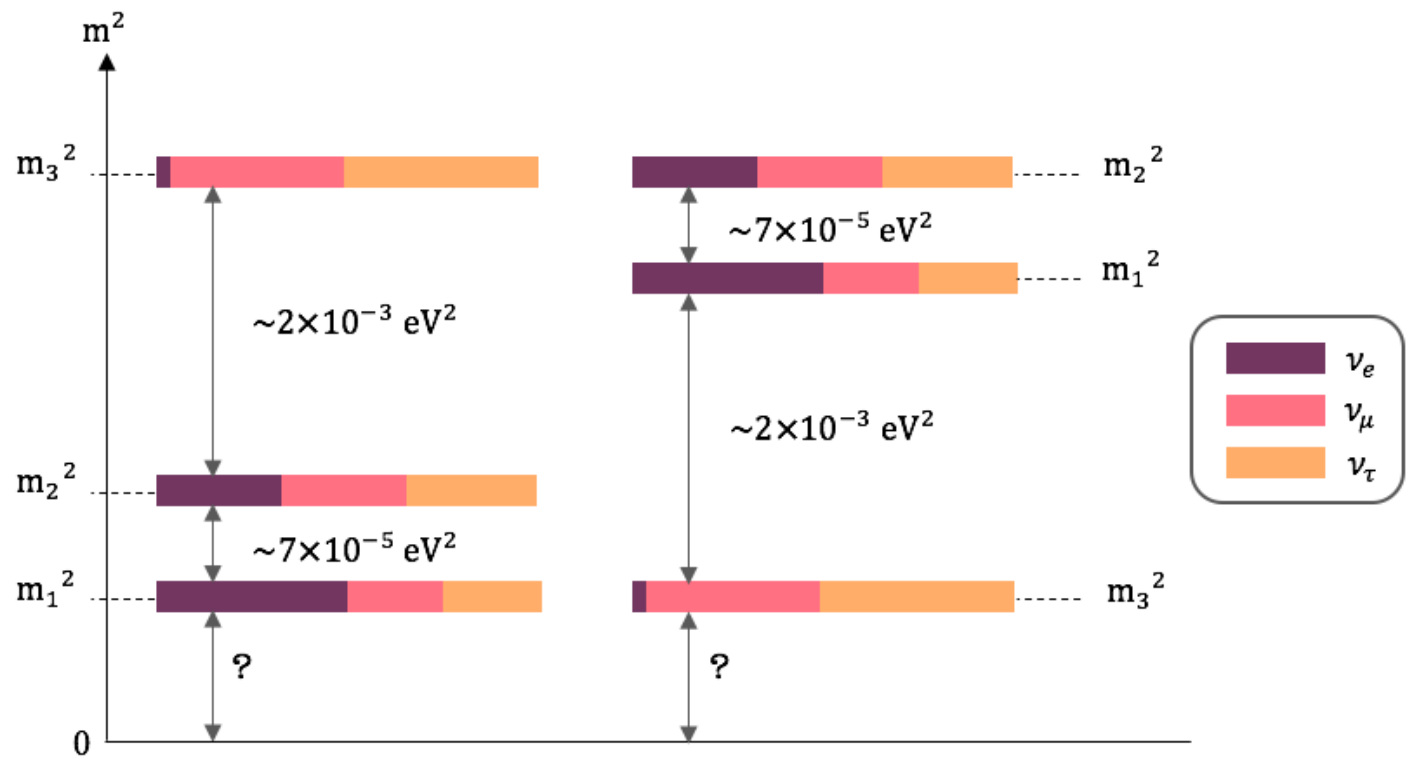

Figure 3.2: While $m_{2}^{2}>m_{1}^{2}$, it is presently unknown whether $m_{3}^{2}$ is heavier or lighter than the other two, referred to as normal (left panel) or inverted (right panel) mass squared ordering. Moreover the value of the lightest neutrino mass is currently unknown indicating by a question mark (?) in each case.

\subsection{Dirac and Majorana Neutrino Masses}

In this section, we discuss the nature of massive neutrinos which will have very crucial impact on the understanding of the origin of neutrino masses and mixings. For a fermion field, we have a free Lagrangian

$$
\mathcal{L}=i \bar{\psi} \gamma^{\mu} \partial_{\mu} \psi-m \bar{\psi} \psi
$$

It is well-known that the term $m \bar{\psi} \psi$ is the mass of the fermion. However, Lorentz invariance allows another kind of mass terms which is $\psi^{T} C^{-1} \psi$, where $C$ is the charge conjugate matrix. Under $\psi \rightarrow e^{i \alpha} \psi$ transformation or a so-called $U(1)_{e m}$ symmetry, the first mass term is invariant whereas the second one is not. We call the first mass term Dirac mass and the second one Majorana mass. Charged fermions including leptons $(e, \mu, \tau)$ and quarks $(\mathrm{u}, \mathrm{d}, \mathrm{c}, \mathrm{s}, \mathrm{t}, \mathrm{b})$ must be Dirac fermions because they have non-zero charges. However with an electrically neutral particle as the neutrino, 
$U(1)_{e m}$ symmetry does not force it to be Dirac. It is generally possible that neutrinos can be either Dirac or Majorana type and therefore possess Dirac or Majorana masses.

\subsubsection{Dirac neutrino mass}

If neutrinos are Dirac type then neutrinos and antineutrinos are distinctive. In this case, they carry different lepton numbers i.e. $L(\nu)=1$ and $L(\bar{\nu})=-1$. Therefore, the total lepton number $L=L_{e}+L_{\mu}+L_{\tau}$ is a conserved quantity in particle interactions. Dirac neutrino masses are obtained as similarly as the SM quark and charged lepton masses. Once we add the right-handed neutrino $\nu_{R}$ to the SM, we can write a gauge-invariant Yukawa coupling to the SM Higgs field $\Phi$ as in an interaction term

where $l_{e L}=\left(\begin{array}{c}\nu_{\nu_{e}} \bar{l}_{e L} \tilde{\Phi} \nu_{e R}+\text { h.c. } \\ e_{L}\end{array}\right), \tilde{\Phi}=i \tau_{2} \Phi^{*}=\left(\begin{array}{c}\phi^{0 *} \\ -\phi^{-}\end{array}\right)$and $\langle\tilde{\Phi}\rangle=\left(\begin{array}{c}\frac{v}{\sqrt{2}} \\ 0\end{array}\right)$. The Dirac neutrino mass term, therefore, can be cast in the form

$$
g_{\nu_{e}} \frac{v}{\sqrt{2}} \bar{\nu}_{e L} \nu_{e R}
$$

and electron neutrino obtains its Dirac mass

$$
m_{\nu_{e}}^{\text {Dirac }}=g_{\nu_{e}} \frac{v}{\sqrt{2}}
$$

Similarly we can get Dirac masses for other neutrinos. If the Dirac mass is the only source of mass one can deduce the Yukawa coupling $g_{\nu}<\mathcal{O}\left(10^{-11}\right)$ since the mass of neutrinos is about $\mathcal{O}(\mathrm{eV})$ and $v \approx 246 \mathrm{GeV}$. Although the value of Yukawa coupling is mathematically acceptable, it does not make sense in Physics because such a small number is highly unnatural. Nevertheless it is possible to have $g_{\nu} \sim \mathcal{O}\left(10^{-11}\right)$ set in dynamically, for example, by a symmetry in which $g_{\nu}=0$ at the tree level, or through new concepts such as extra spatial dimensions. 


\subsubsection{Majorana neutrino mass}

If neutrinos are their own anti-particles, it is said to be Majorana type neutrinos. It is noticed that if the fermion field $\psi$ satisfied the condition of being self charge conjugate, i.e.

$$
\psi=\psi^{c} \equiv C \bar{\psi}^{T}
$$

then the mass term $\bar{\psi} \psi$ is driven to the mass term $\psi^{T} C^{-1} \psi$. Since the neutrino and antineutrino are now the same particle, they have the same lepton number and therefore, there are no conserved lepton numbers in this case. In fact Majorana masses violate total lepton number conservation by two units $\Delta L=2$. One can write a Majorana mass term of the form

$$
M_{R} \nu_{R}^{T} C \nu_{R}
$$

After all right-handed neutrinos are gauge singlets and, hence, nothing prevents them from getting (large) Majorana mass term. One can generate Majorana mass via the interaction

$$
y_{M} \Delta l_{L} l_{L}
$$

where $l_{L}$ are lepton doublets and $\Delta$ is a Higgs triplet under $S U(2)_{L}$. Another way could be the coupling between 2 lepton doublets and 2 Higgs doublets, $H$, in which Majorana neutrino mass can result from some additional dimension-5 operators 46,56

$$
\frac{1}{2} H l_{L}^{T} \kappa H l_{L}
$$

Here $\kappa$ has the dimension $[\text { mass }]^{-1}$.

At this moment, the nature of neutrino mass has not been revealed yet. Experiments of the flavor neutrino oscillations cannot tell whether neutrinos are Dirac or Majorana fermions. The only possibility is to investigate the total lepton number violating processes through which we can address the question of the Majorana nature of neutrinos. Until now, the neutrinoless double beta decay $(0 \nu \beta \beta)$ has been the most advanced experimentally and theoretically 5759 . This process 
is written in a simple form

$$
(A, Z) \rightarrow(A, Z+2)+e^{-}+e^{-}
$$

and described by a Feynman diagram at the quark level as shown in Fig. (3.3). If there is a positive

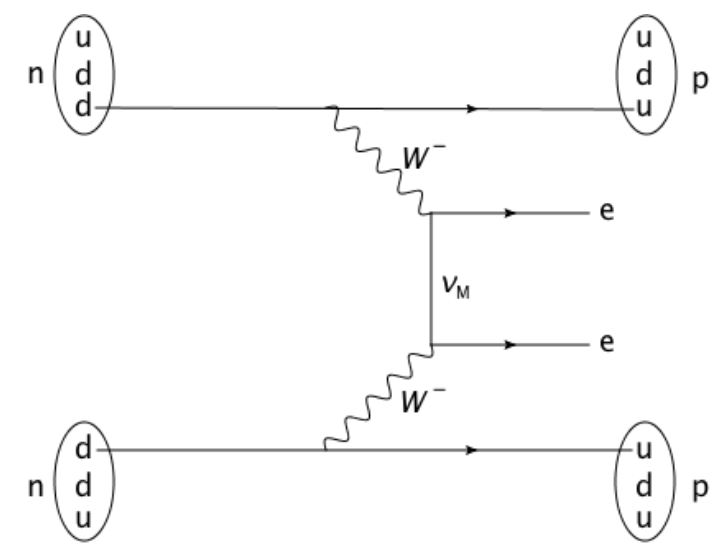

Figure 3.3: Diagram of the $0 \nu \beta \beta$ process due to the exchange of massive Majorana neutrinos in $n+n \rightarrow p+p+2 e$, here denoted generically by $\nu_{M}$.

signal of the above process it will lead to an establishment of Majorana neutrinos, although it will not be easy to determine the absolute mass of the neutrino. Further experiments are needed to unravel the mysteries of the neutrinos:

- The origin of neutrino masses: why they are so light?

- Are neutrinos Dirac or Majorana?

- Is there CP Violation in the neutrino sector?

- The origin of mass hierarchy and mixings?

Apparently, SM cannot give us satisfactory answers for these open questions that puzzle scientists for years. Thus one must go beyond the edge and strive for New Physics to explain observed evidences for neutrino masses. Whilst many theoretical attempts lead to small neutrino masses of both Majorana and Dirac types, we will focus on the possibility that there is a heavy right-handed 
neutrino resulting in tiny masses for light neutrinos. A celebrated example of such a mechanism is the "Seesaw mechanism" which will be discussed in the following section.

\subsection{Seesaw mechanism of neutrino masses}

In the theory of particle physics, the seesaw mechanism generically models the observed neutrino masses $\sim \mathcal{O}(e V)$ which are millions of times lighter than quarks and charged leptons masses. In this section we will briefly review three popular types of models called Type-I, Type-II, and Type-III Seesaw. Each of them concerns different extensions of the SM but has the same goal which is to generate two mass scales - one heavier than the other. The simplest one is type-I seesaw in which right-handed neutrinos are added and act as singlets under $S U(2)$. The type-II seesaw uses a scalar triplet Higgs to couple with a fermion bilinear. The last one, type-III seesaw, achieves its goal by adding one real fermion triplet to the SM. The detailed discussion mainly follows [46, 60].

\subsubsection{Type-I Seesaw}

By introducing right-handed neutrino $\nu_{R}$ for each family of fermions, we now have both of Dirac and Majorana mass terms as seen in Eq. (3.39) and (3.42)

$$
\mathcal{L}=m_{D}\left(\bar{\nu}_{L} \nu_{R}+\bar{\nu}_{R} \nu_{L}\right)+M_{R} \nu_{R}^{T} C \nu_{R}+\text { h.c. }
$$

We can set $\nu \equiv \nu_{L}$ and $N \equiv C \nu_{R}^{*}$ and obtain the mass matrix for $\nu$ and $N$ as

$$
\left(\begin{array}{ll}
\nu^{T} & N^{T}
\end{array}\right)\left(\begin{array}{cc}
0 & m_{D} \\
m_{D}^{T} & M_{R}
\end{array}\right) C\left(\begin{array}{c}
\nu \\
N
\end{array}\right)
$$

Several scenarios can be considered in this case:

- $M_{R} \ll m_{D}$ : neutrinos are essentially Dirac. 
- $M_{R} \simeq m_{D}$ : neutrinos are combination of Dirac and Majorana types.

- $M_{R} \gg m_{D}$ : neutrinos are essentially Majorana.

It would be interesting to consider the scenario in which $M_{R} \gg m_{D}$ because $M_{R}$ can be larger than the electroweak scale $\Lambda_{E W}$. Diagonalizing the neutrino mass matrix in Eq. (3.47) gives us eigenvalues

$$
\begin{array}{ll}
M_{N}=M_{R} & \text { (large mass scale) } \\
M_{\nu}=-m_{D}^{T} M_{R}^{-1} m_{D} & \text { (small mass scale) } .
\end{array}
$$

As an example, in one family if we take $m_{D} \sim M_{W}$ (mass of the $W$ boson) and $M_{R} \sim M_{G U T}$ then $m_{\nu} \sim 10^{-3} \mathrm{eV}$ which can account for solar neutrino masses. Having said that such $\nu_{R}$ 's are too heavy so one can not produce and detect the $\nu_{R}$ 's at the LHC, or the seesaw mechanism is not testable. It is also noticed that the number of added $\nu_{R}$ 's determines the number of massive light neutrinos. Therefore, we must add, at least, two $\nu_{R}$ 's to compensate for both solar and atmospheric neutrino mass differences. The diagram shown in Fig. (3.4) gives us a clearer visualization of the type-I seesaw mechanism.

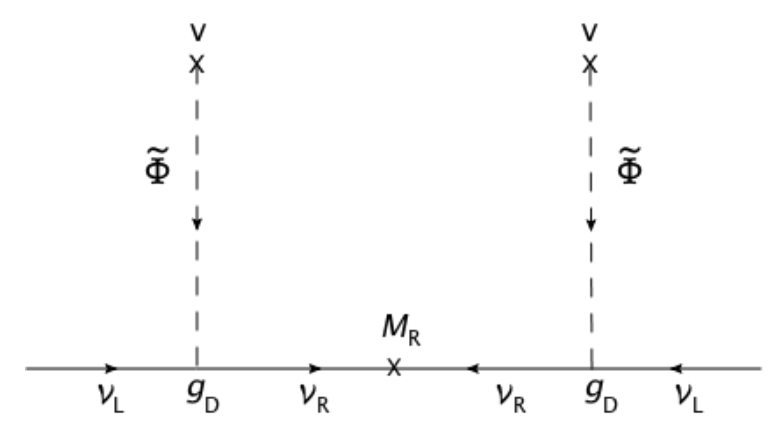

Figure 3.4: Diagrammatic representation for type-I seesaw mechanism. 


\subsubsection{Type-II Seesaw}

Another possibility to generate two mass scales is adding a $S U(2)_{L}$ triplet Higgs with $Y / 2=1$ which plays the same role as the $\nu_{R}$ 's in the type-I seesaw. The Higgs is defined as

$$
\Delta_{L} \equiv \frac{1}{\sqrt{2}} \vec{\Delta}_{L} \cdot \vec{\tau}=\left(\begin{array}{cc}
\Delta^{+} / \sqrt{2} & \Delta^{++} \\
\Delta^{0} & -\Delta^{+} / \sqrt{2}
\end{array}\right)
$$

where $\vec{\Delta}_{L}=\left(\Delta^{++}, \Delta^{+}, \Delta^{0}\right)$ and $\vec{\tau}=\left(\tau^{+} \equiv \frac{\sigma_{1}+i \sigma_{2}}{\sqrt{2}}, \tau^{0} \equiv \sigma_{3}, \tau^{-} \equiv \frac{\sigma_{1}-i \sigma_{2}}{\sqrt{2}}\right)$. Here $\sigma_{1,2,3}$ are Pauli matrices. From the Lagrangian

$$
\mathcal{L}=g_{\Delta} l_{L}^{T} C \sigma_{2} \Delta_{L} l_{L}+\text { h.c. }
$$

$\nu_{L}$ 's acquire Majorana masses $m_{\nu}=g_{\Delta} v_{\Delta}$ through the VEV of the neutral component of the scalar triplet:

$$
\left\langle\Delta_{L}\right\rangle=\left(\begin{array}{cc}
0 & 0 \\
v_{\Delta} & 0
\end{array}\right)
$$

The following cubic scalar potential

$$
\Delta V=\mu_{\Delta} \Phi^{T} \sigma_{2} \Delta_{L}^{*} \Phi+M_{\Delta}^{2} \operatorname{Tr} \Delta_{L}^{\dagger} \Delta_{L}+\ldots
$$

results in the VEV of the scalar Higgs of the form $\left\langle\Delta_{L}\right\rangle \simeq \frac{\mu_{\Delta} v^{2}}{M_{\Delta}^{2}}$. It is noticed that the VEV is induced by the term linear in $\Delta_{L}$ in the scalar potential and is highly suppressed if $M_{\Delta} \gg v$ causing neutrinos are naturally light in this case. Therefore, one does not need to fine-tune the couplings $g_{\Delta}$ or $\mu_{\Delta}$ in order to achieve the tiny neutrino masses. The diagram in Fig. (3.5) will represent the idea of type-II seesaw mechanism in such a vivid way.

\subsubsection{Type-III Seesaw}

As far as we go, type-I and type-II seesaw have done a good job on explaining the light neutrino masses with minimal extensions of the SM. One may look back the Yukawa interaction 


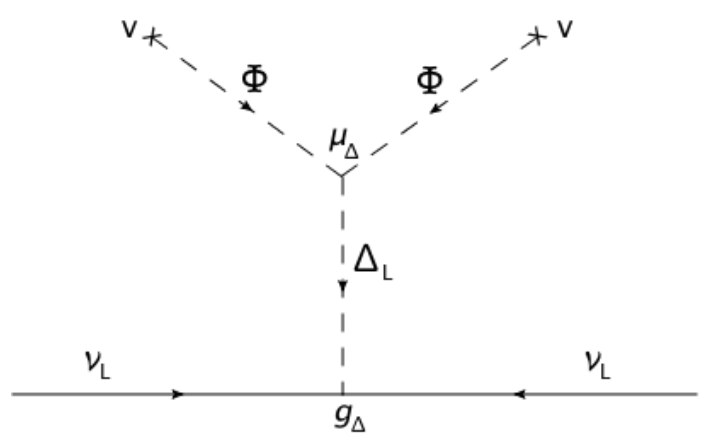

Figure 3.5: Diagrammatic representation for type-II seesaw mechanism.

(3.46) for right-handed neutrinos in type-I seesaw and realize that we can do exactly the same thing for a new fermion triplet $(Y / 2=0) \vec{T}_{F}=\left(T_{F 1}, T_{F 2}, T_{F 3}\right)$ within one family. As discussed in the type-I seesaw, we will need at least two fermion triplets to provide two massive light neutrinos. A similar Feynman diagram as in Fig. (3.6) will be made for such new fermions whose Lagrangian (for one generation as an example) is

$$
\mathcal{L}=g_{T} l^{T} C \vec{\tau} \cdot \vec{T}_{F} \Phi+M_{T} \vec{T}_{F}^{T} C \vec{T}_{F}
$$

which gives the mass matrix for neutrino

$$
\left(\begin{array}{cc}
0 & g_{T} v / \sqrt{2} \\
g_{T}^{T} v / \sqrt{2} & M_{T}
\end{array}\right)
$$

For $M_{T} \gg v$ we, again, obtain the light neutrino mass after a $S U(2) \times U(1)$ symmetry breaking in the form of

$$
m_{\nu}=-g_{T}^{T} \frac{1}{M_{T}} g_{T} v^{2}
$$

At this point, a few remarks are in order:

- Under the assumption of single type of new particles added to the SM, three types of seesaw mechanism satisfy all possibilities of replicating dimension-5 operator as in Eq. 3.44.

- If the new scales $M_{R}, M_{\Delta}$, and $M_{T}$ are huge and not accessible to experiments then it is hard to test these seesaw mechanisms. 


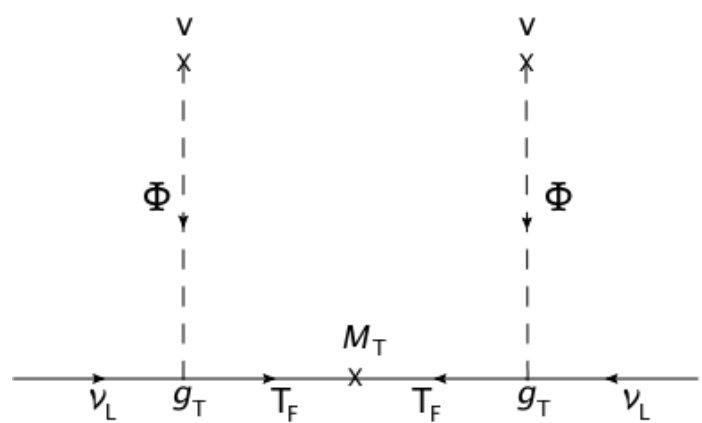

Figure 3.6: Diagrammatic representation for type-III seesaw mechanism.

As a result, it motivated many theorists to step in and build models in order to have Majorana neutrino mass and seesaw mechanism accessible at the accelerators. One of those will be introduced in next section.

\subsection{Left-Right symmetric model}

A more elegant approach to the neutrino Majorana mass term is the so-called Left-Right (L-R) symmetric model 61, 62 constructed under $S U(2)_{L} \times S U(2)_{R} \times U(1)_{B-L}$ gauge group. At some energy scale $v_{R}$, the $S U(2)_{R} \times U(1)_{B-L}$ gauge symmetry is spontaneously broken down to $U(1)_{Y}$ whose generators are now

$$
Y=T_{3 R}+Y_{B-L} \quad \text { and } \quad Q=T_{3 L}+Y
$$

The L-R symmetric model has the right-handed neutrinos as a part of $S U(2)_{R}$ doublets along with the right-handed charged leptons as oppose to the type-I seesaw mechanism. Notice that $\nu_{R}$ 's remain singlets under $S U(2)_{L}$. The particle content as well as particles' quantum numbers under the $S U(2)_{L} \times S U(2)_{R} \times U(1)_{B-L}$ gauge group are listed as follows 
$\circledast$ Fermions (in generic symbols)

$$
\begin{aligned}
& l_{L}=\left(\begin{array}{c}
\nu_{L} \\
e_{L}
\end{array}\right)=\left(2,1, Y_{B-L}=-1 / 2\right), \\
& l_{R}=\left(\begin{array}{c}
\nu_{R} \\
e_{R}
\end{array}\right)=\left(1,2, Y_{B-L}=-1 / 2\right), \\
& q_{L}=\left(\begin{array}{c}
u_{L} \\
d_{L}
\end{array}\right)=\left(2,1, Y_{B-L}=1 / 6\right), \\
& q_{R}=\left(\begin{array}{c}
u_{R} \\
d_{R}
\end{array}\right)=\left(1,2, Y_{B-L}=1 / 6\right) .
\end{aligned}
$$

$\circledast$ Higgs fields

$$
\begin{aligned}
\Phi & =\left(\begin{array}{cc}
\phi_{1}^{0} & \phi_{2}^{+} \\
\phi_{1}^{-} & \phi_{2}^{0}
\end{array}\right)=\left(2,2, Y_{B-L}=0\right) \\
\Delta_{L, R} & =\left(\begin{array}{cc}
\frac{\Delta_{L, R}^{+}}{\sqrt{2}} & \Delta_{L, R}^{++} \\
\Delta_{L, R}^{0} & -\frac{\Delta_{L, R}^{+}}{\sqrt{2}}
\end{array}\right), \\
\text { where } \Delta_{L} & =\left(3,1, Y_{B-L}=1\right), \Delta_{R}=\left(1,3, Y_{B-L}=1\right) .
\end{aligned}
$$

For the purpose of illustrating Dirac and Majorana mass terms of right-handed neutrinos, we concentrate on the lepton sector and skip $\Delta_{L}$. We have the VEVs

$$
\langle\Phi\rangle=\left(\begin{array}{cc}
\kappa & 0 \\
0 & \kappa^{\prime}
\end{array}\right), \quad\left\langle\Delta_{R}\right\rangle=\left(\begin{array}{cc}
0 & 0 \\
v_{R} & 0
\end{array}\right) \text {. }
$$

Therefore from the Lagrangian

$$
\begin{aligned}
\mathcal{L}_{L-R} & =h_{1} \bar{l}_{L} \Phi l_{R}+h_{2} \bar{l}_{L} \tilde{\Phi} l_{R}+\text { h.c. } \\
& +i g_{\Delta_{R}} l_{R}^{T} \sigma_{2} \tau_{2} \Delta_{R} l_{R}+\text { h.c. }
\end{aligned}
$$

We obtain the neutrino Majorana and Dirac mass terms of the forms $\left(g_{\Delta_{R}} v_{R}\right) \nu_{R}^{T} \sigma_{2} \nu_{R}$ and $\left(h_{1} \kappa+\right.$ $\left.h_{2} \kappa^{\prime}\right)\left(\nu_{L}^{\dagger} \nu_{R}+\nu_{R}^{\dagger} \nu_{L}\right)$ respectively. 
This model also extends the number of gauge bosons by $W_{R}^{ \pm}$and $Z_{R}$ of the $S U(2)_{R}$ gauge symmetry. So far, the breaking scale has had a lower bound of $\Lambda_{E W}=246 \mathrm{GeV}$ and no current upper bound. One expects to validate this model by its primary signals $\bar{u}+d \rightarrow W_{R}^{-} \rightarrow \nu_{R}+l$ at the LHC. Regarding these searches, the $W_{R}$ bosons have masses above $\sim 3 \mathrm{TeV}$.

\subsection{Overall remarks}

- Neutrino oscillation evidences from solar and atmospheric neutrino anomalies open a door to new physics beyond the SM. There are, at least, two in three light neutrinos have tiny but non-zero masses.

- Neutrino oscillations arise from a mixture between the flavor and mass eigenstates of neutrinos via the PMNS matrix.

- Until now, the nature of neutrinos which is Dirac or Majorana type remains unrevealed. If $\nu$ is Dirac then why the coupling constant $g_{\nu} \leq \mathcal{O}\left(10^{-11}\right)$. If $\nu$ is Majorana then the lighter of the 2 states can be very light.

- Many theoretical models were built to explain the tininess of neutrino masses but so far none of them have been validated.

- Although the seesaw mechanisms gain their theoretical recognitions when leading us to small neutrino masses they involve very large-scale new particles beyond SM. Such particles are too heavy to be produced and detected at the LHC or other colliders. 


\section{Chapter 4}

\section{The Minimal EW- $\nu_{R}$ Model}

\subsection{Motivation}

The mystery of the origin of neutrino masses and mixings remains unrevealed despite the fact that we had several mechanisms providing possible explanations for it. The seesaw mechanisms that we have already reviewed in the previous chapter were the most successful ones. However, in a general seesaw the right-handed neutrinos are singlets under $S U(2)_{L} \times U(1)_{Y}$ and the Majorana neutrino masses, $M_{R}$ can be of the order of Grand Unified Theory (GUT) mass scale which is $10^{15-16} \mathrm{GeV}$ naturally. Such a huge mass will not be accessible at the colliders, e.g. the LHC, and therefore the corresponding seesaw mechanism is not testable.

On the other hand the L-R symmetric model discussed previously has suggested a possibility of producing and detecting the right-handed neutrinos at the LHC by making $\nu_{R}$ 's doublets under an extended $S U(2)_{R}$ gauge group (still remain singlets under the $\left.S U(2)_{L}\right)$. However the associated $W_{R}$ gauge boson masses have been bounded from below at around $3 \mathrm{TeV}$ [63].

The above facts give rise to the following questions

- Is it possible to make the seesaw testable? 
- Can $M_{R}$ be of the order of the electroweak scale, $\Lambda_{E W} \sim 246 \mathrm{GeV}$ so one could directly look for its signatures at future colliders?

- Can we have things done by keeping the SM gauge group $S U(3)_{C} \times S U(2)_{L} \times U(1)_{Y}$ and having no more forces added?

- Can neutrinos be non-sterile or fertile under SM gauge group?

In 2007, P.Q. Hung has proposed a so-called Electroweak-scale Right-handed Neutrino (EW- $\left.\nu_{R}\right)$ Model 64 which satisfied all of the above criteria. From that moment, it opens a new whole fertile land for the study of neutrino.

The motivation of introducing mirror fermions in [64] was manifold. First of all, it is aesthetically satisfactory to have parity restoration at a higher energy scale while the maximal parity violating interaction (V-A interaction) in SM can be emerged from spontaneous symmetry breaking. This is one of the main reasons for various left-right symmetric models in the literature 114 117]. Secondly, electroweak phase transition is intrinsically non-perturbative so it is important to study non-perturbative effects in SM by discretizing it on the lattice. We have learned from the no-go theorem proved by Nielsen and Ninomiya [118 that the number of left-handed Weyl fields must be equal to that of right-handed Weyl fields, coupled to the same chiral gauge fields. Since the SM is chiral and only left-handed doublets coupled to $W$ 's, one cannot put SM on the lattice. Sophisticated techniques like using Wilson fermion, Ginsparg-Wilson fermion, staggered fermion, or domain wall fermion etc., which by violating at least one of the assumptions in the no-go theorem to get rid of the unwanted species, are often employed to handle this problem in practice. For new physics model builders, it is attractive to add mirror fermions to the SM which makes the theory becomes vector-like at a higher scale and hence one can avoid the fermion doubling problem if formulating on the lattice. Chiral gauge anomalies will then be cancelled automatically in this class of models. The third motivation is the electroweak scale non-sterile right-handed neutrinos introduced in [64]. For each generation, the right-handed neutrino is introduced together with a right-handed heavy 
charged fermion partner to form a SM SU(2) doublet. Similarly a left-handed heavy mirror charged lepton will be introduced for each right-handed SM charged lepton. Majorana masses can then be given to these right-handed neutrinos via the vacuum expectation value (VEV) of a Higgs triplet with hypercharge $Y=2$ with mass at the electroweak scale, rather than the grand unification scale

in the usual scheme. Tiny Dirac masses can also be given via small VEVs of Higgs singlets with $Y=0$. This is the electroweak scale see-saw mechanism in mirror fermion model which is testable at the LHC 74,119 .

In the following sections we will first see how the EW- $\nu_{R}$ model was built step-by-step. Secondly we will investigate a SSB of the $S U(2)_{L} \times U(1)_{Y}$ to $U(1)_{E M}$ in this model, resulting in masses of fermions, especially that of Dirac and Majorana neutrinos. The last section will mainly focus on phenomenological constraints of the $\mathrm{EW}-\nu_{R}$ model.

\subsection{Definition, gauge group, and particle content}

$\mathrm{EW}-\nu_{R}$ is a model in which the right-handed neutrinos $\nu_{R}$ have Majorana mass of $\mathcal{O}\left(\Lambda_{E W}\right)$ naturally and they are non-sterile under $S U(2)_{L} \times U(1)_{Y}$. We are able to obtain such a seesaw mechanism within the SM gauge group

$$
G_{S M}=S U(3)_{c} \times S U(2)_{L} \times U(1)_{Y}
$$

by increasing its fermion as well as its Higgs content, all of which are listed below. In order to do so we introduce and add a so-called "Mirror fermions". The formation of mirror fermions follows a "rule" in which for every SM left-handed doublets there is a corresponding right-handed mirror doublet and for every SM right-handed singlet there is a corresponding left-handed mirror singlet.

Let us first start with building the particle content. We take, for example, the first generation 
of the lepton sector of the SM:

$$
l_{L}=\left(\begin{array}{c}
\nu_{L} \\
e_{L}
\end{array}\right), e_{R}
$$

which are a $S U(2)_{L}$ doublet and singlet, respectively. We have learned from the interaction between SM fermions and scalar doublet $\Phi$ that the product $\bar{l}_{L} \Phi$ or $\bar{l}_{L} \tilde{\Phi}$ transforms as a singlet under $S U(2)_{L}$. On the other hand, the general seesaw mechanism tells us the $\nu_{R}$ is also a singlet of $S U(2)_{L}$ and, therefore, can get a Dirac mass by Yukawa coupling to the above products. The down side of this, again, is a huge Majorana mass of $\nu_{R}$. Hence, this EW- $\nu_{R}$ model arranges $\nu_{R}$ into a doublet along with a right-handed mirror lepton as

$$
l_{R}^{M}=\left(\begin{array}{c}
\nu_{R} \\
e_{R}^{M}
\end{array}\right), e_{L}^{M}
$$

where the superscript ' $M$ ' stands for 'mirror fermions'. The charged left-handed singlet mirror lepton, $e_{L}^{M}$ is the mirror counterpart of $e_{R}$. It is emphasized that mirror leptons are totally different from the ordinary SM leptons. They can have the same quantum numbers but not necessarily have the same masses. In this case, anomaly cancellation runs entirely within the lepton sector including both SM and mirror leptons so one can reassure that we do not encounter any trouble with adding such mirror particles [64]. Because of the existence of right-handed mirror doublets coupling to the same SM gauge fields, the gauge group becomes

$$
G_{S M} \rightarrow S U(3)_{c} \times S U(2)_{W} \times U(1)_{Y}
$$

where 'W' stands for 'weak'. From hereon, we will use it as our official gauge group.

On the other hand, it is noticed that anomaly cancellation requires the existence of mirror quarks. Therefore we must add them into the model by making same arguments as with the lepton sector. As a result, the first quark generation appears as

$$
\mathrm{SM}: q_{L}=\left(\begin{array}{c}
u_{L} \\
d_{L}
\end{array}\right), u_{R}, d_{R} ; \quad \text { Mirror: } q_{R}^{M}=\left(\begin{array}{c}
u_{R}^{M} \\
d_{R}^{M}
\end{array}\right), u_{L}^{M}, d_{L}^{M} .
$$


One can straightforwardly generalize the above discussion for three families of leptons and quarks. Hence we will use the notations in Eq. 4.2, (4.3) and 4.5 as generic ones to refer to all three fermion families from hereon.

On the scalar sector side, so far the minimal $\mathrm{EW}-\nu_{R}$ model contains one SM Higgs doublet $\Phi$ in order to generate masses for charged SM fermions. Since the subject of this model is neutrino masses, we wish to find scalar fields giving rise to Dirac and Majorana mass terms. We notice that a bilinear such as $\bar{l}_{L} l_{R}^{M}=\bar{\nu}_{L} \nu_{R}+\bar{e}_{L} e_{R}^{M}$ can transform as a singlet under $S U(2)_{L}$. Therefore in order to acquire the Dirac neutrino mass, let us assume the existence of a singlet scalar field $\phi_{S}$ carrying a hypercharge $Y / 2=0$ 64]. Its coupling with the fermion bilinear is

$$
\mathcal{L}_{S}=g_{S l} \bar{l}_{L} \phi_{S} l_{R}^{M}+\text { h.c. }=g_{S l}\left(\bar{\nu}_{L} \nu_{R}+\bar{e}_{L} e_{R}^{M}\right) \phi_{S}+\text { h.c. }
$$

Now let us turn to the $\nu_{R}$ Majorana mass term for whom fermion bilinear $l_{R}^{M, T} \sigma_{2} l_{R}^{M}(Y / 2=-1)$ transforms as either a singlet or a triplet under $S U(2)_{L} \times U(1)_{Y}$. We see that the Higgs singlet cannot be the candidate in this case since charge conservation is violated. This leaves us with the choice of a scalar triplet Higgs field $\tilde{\chi}=(3, Y / 2=+1)$ having the form

$$
\tilde{\chi}=\frac{1}{\sqrt{2}} \vec{\tau} \cdot \vec{\chi}=\left(\begin{array}{cc}
\frac{1}{\sqrt{2}} \chi^{+} & \chi^{++} \\
\chi^{0} & -\frac{1}{\sqrt{2}} \chi^{+}
\end{array}\right) .
$$

The role of these Higgs fields in generating fermion masses, especially neutrino masses will be discussed carefully in next sections.

\subsection{Dirac and Majorana neutrino masses in the EW- $\nu_{R}$ model}

- Dirac Neutrino Mass

Let us recall the coupling of singlet scalar field $\phi_{S}$ from Eq. (4.6). With the VEV $\left\langle\phi_{S}\right\rangle=v_{S}$, we get the Dirac neutrino masses

$$
m_{\nu}^{D}=g_{S l} v_{S}
$$


If this is the case in which right-handed neutrinos have pure Dirac mass terms, one could set a small $v_{S}$ value to make the Dirac mass naturally small. However, the $Z$-boson decay width [42] forces the right-handed neutrinos to be heavier than half of the $Z$ otherwise the number of light neutrinos will exceed three. Therefore such an ideal is ruled out and we must obtain the neutrino mass via the regular seesaw mechanism.

- Majorana Neutrino Mass

With the complex scalar Higgs triplet $\tilde{\chi}$ being chosen in (4.7), one can write a gauge invariant Yukawa coupling:

$$
\begin{aligned}
L_{M}= & g_{M} l_{R}^{M, T} \sigma_{2} \tau_{2} \tilde{\chi} l_{R}^{M} \\
= & g_{M} \nu_{R}^{T} \sigma_{2} \nu_{R} \chi^{0}-\frac{1}{\sqrt{2}} \nu_{R}^{T} \sigma_{2} e_{R}^{M} \chi^{+} \\
& -\frac{1}{\sqrt{2}} e_{R}^{M, T} \sigma_{2} \nu_{R} \chi^{+}+e_{R}^{M, T} \sigma_{2} e_{R}^{M} \chi^{++} .
\end{aligned}
$$

With the VEV of $\left\langle\chi^{0}\right\rangle=v_{M}$, 4.9 gives rise to the Majorana mass of the right-handed neutrino:

$$
M_{R}=g_{M} v_{M}
$$

Again, one would like to have $M_{R} \geq M_{Z} / 2$ in order to preserve the highly precise $Z$ decay width measurement.

Furthermore, it is also possible to have such a Majorana mass of the left-handed neutrino through a Yukawa coupling $g_{L} l_{L}^{T} \sigma_{2} \tau_{2} \tilde{\chi} l_{L}$. The issue is that this term could potentially ruin all efforts to promote the seesaw mechanism involving one very small - one very large neutrino mass [64]. To get rid of this term, 64 imposed a global symmetry $U(1)_{M}$ such that

$$
\begin{aligned}
& \left(l_{R}^{M}, q_{R}^{M}\right) \rightarrow e^{i \theta_{M}}\left(l_{R}^{M}, q_{R}^{M}\right), \\
& \tilde{\chi} \rightarrow e^{-2 i \theta_{M}} \tilde{\chi}, \quad \phi_{S} \rightarrow e^{-i \theta_{M}} .
\end{aligned}
$$

and all other particles are singlets of $U(1)_{M}$. Although the symmetry can abandon the Majorana mass $M_{L}$ at tree level, it cannot do the same at the one-loop level. The mass 
appears to be

$$
M_{L}=\lambda \frac{1}{16 \pi^{2}} \frac{\left(m_{\nu}^{D}\right)^{2}}{M_{R}} \ln \frac{M_{R}}{M_{\phi_{S}}},
$$

where $\lambda$ and $M_{\phi_{S}}$ are the quadratic coupling and the mass of the Higgs singlet $\phi_{S}$. If $\lambda<1$ then $M_{L}$ is smaller than a typical seesaw light mass $\left(m_{\nu}^{D}\right)^{2} / M_{R}$ by at most two orders of magnitude.

Having said that we still keep this $M_{L}$ term in the Majorana mass matrix for a general purpose:

$$
\mathcal{M}=\left(\begin{array}{cc}
M_{L} & m_{\nu}^{D} \\
m_{\nu}^{D} & M_{R}
\end{array}\right)
$$

where $m_{\nu}^{D}, M_{R}$ and $M_{L}$ are given in Eqs. 4.8, 4.10, and 4.12 respectively. If the Yukawa coupling $g_{S l} \sim \mathcal{O}\left(g_{M}\right)$ and $v_{M} \gg v_{S}$ we have the following eigenvalues

$$
m_{\nu}=M_{L}-\frac{\left(m_{\nu}^{D}\right)^{2}}{M_{R}}=-\frac{g_{S l}^{2}}{g_{M}} \cdot \frac{v_{S}}{v_{M}} \cdot v_{S}(1-\epsilon) \text { and } M_{R}
$$

with $\epsilon<10^{-2}$. To accommodate the need of $M_{R} \approx \Lambda_{E W} \approx 246 \mathrm{GeV}$ regarding the constraint on light neutrino masses $m_{\nu} \leq 0.23 \mathrm{eV}$ [4], we can alternate the coupling $g_{S l}$ resulting in corresponding values of $v_{S}$. For example, $v_{S} \sim 10^{5-6} \mathrm{eV}$ with $g_{S l} \sim \mathcal{O}(1)$ and $v_{S} \sim \Lambda_{E W}$ with $g_{S l} \sim \mathcal{O}\left(10^{-6}\right)$ 64. One may ask about the hierarchy of $v_{S} / \Lambda_{E W} \sim 10^{-6}$. Apparently it is not as severe as the one related to the GUT-scale $\left(\sim \mathcal{O}\left(10^{6}\right) \mathrm{GeV}\right)$. More detailed discussion can be found in 64 .

\subsection{Charged fermion masses (lepton and quark)}

As mentioned in the previous section, in order to generate charged fermion masses (say, leptons) we will need to consider the SM Yukawa couplings to the Higgs doublet $\Phi$ as follows:

$$
\begin{aligned}
\mathcal{L}_{Y_{S M}} & =g_{l} \bar{l}_{L} \Phi e_{R}+\text { h.c. }, \\
\mathcal{L}_{Y_{M}} & =g_{l}^{M} \bar{l}_{R}^{M} \Phi e_{L}^{M}+\text { h.c. } .
\end{aligned}
$$


After SSB, $\Phi$ acquires its $\operatorname{VEV}\langle\Phi\rangle=\left(0, v_{2} / \sqrt{2}\right)^{T}$. Note that the $\operatorname{VEV}$ of $\Phi$ is written in terms of $v_{2}$ which is different from the total $v \approx 246 \mathrm{GeV}$. From Eqs. 4.6, 4.15, 4.16 one obtains a mass matrix for charged SM and mirror leptons

$$
\mathcal{M}_{l}=\left(\begin{array}{cc}
m_{l} & m_{\nu}^{D} \\
m_{\nu}^{D} & m_{l^{M}}
\end{array}\right)
$$

where $m_{l}=g_{l} v_{2} / \sqrt{2}$ and $m_{l^{M}}=g_{l}^{M} v_{2} / \sqrt{2}$. Diagonalizing the matrix 4.17 results in two eigenvalues

$$
\tilde{m_{l}}-m_{l}-\frac{\left(m_{\nu}^{D}\right)^{2}}{m_{l^{M}}-m_{l}}, \quad \tilde{m_{l^{M}}}=m_{l^{M}}+\frac{\left(m_{\nu}^{D}\right)^{2}}{m_{l^{M}}-m_{l}} .
$$

By making an assumption $m_{l^{M}} \gg m_{l}$ and using the fact that $m_{\nu}^{D} \ll m_{l}, m_{l^{M}}$ as discussed in the neutrino mass section the eigenvalues in 4.18 become $\tilde{m}_{l} \approx m_{l}$ and $\tilde{m}_{l^{M}} \approx m_{l^{M}}$.

The same exercise can be done for the quark sector by simply replacing: $m_{l} \rightarrow m_{q}, m_{l^{M}} \rightarrow$ $m_{q^{M}}$. Hence, quarks acquire their masses through Yukawa couplings

$$
\begin{aligned}
\mathcal{L}_{S q} & =g_{S q} \bar{q}_{L} \phi_{S} q_{R}^{M}+\text { h.c. }=g_{S q}\left(\bar{u}_{L} u_{R}^{M}+\bar{d}_{L} d_{R}^{M}\right) \phi_{S}+\text { h.c. }, \\
\mathcal{L}_{Q Y_{S M}} & =g_{d} \bar{q}_{L} \Phi d_{R}+g_{u} \bar{q}_{L} \tilde{\Phi} u_{R}+\text { h.c. }, \\
\mathcal{L}_{Q Y_{M}} & =g_{d}^{M} \bar{q}_{R}^{M} \Phi d_{L}^{M}+g_{u}^{M} \bar{q}_{R}^{M} \tilde{\Phi} u_{L}^{M}+\text { h.c. } .
\end{aligned}
$$

Diagonalizing the quark mass matrix gives rise to eigenvalues

$$
\tilde{m}_{q}=m_{q}-\frac{\left(m_{\nu}^{D}\right)^{2}\left(g_{S q} / g_{S l}\right)^{2}}{m_{q^{M}}-m_{q}}, \quad \tilde{m}_{q^{M}}=m_{q^{M}}+\frac{\left(m_{\nu}^{D}\right)^{2}\left(g_{S q} / g_{S l}\right)^{2}}{m_{q^{M}}-m_{q}}
$$

where $q=u, d, \ldots, m_{q}=g_{q} v_{2} / \sqrt{2}$, and $m_{q^{M}}=g_{q}^{M} v_{2} / \sqrt{2}$. Again, if we assume $m_{q^{M}} \gg m_{q}$, one has $\tilde{m}_{q} \approx m_{q}, \tilde{m}_{q^{M}} \approx m_{q^{M}}$.

\section{5 $\rho$ parameter and possible signatures of $\mathbf{E W}-\nu_{R}$ model}

In the SM, the $\rho$ parameter at the tree level which is defined as

$$
\rho \equiv \frac{M_{W}^{2}}{M_{Z}^{2} \cos ^{2} \theta_{W}}
$$


is measured to be 1 to a good precision. Therefore, when introducing new Higgs fields other than $\Phi$ one must make sure that there is a remaining custodial symmetry resulting in $\rho=1$. In Higgs sector, a number of Higgs multiplets $\phi_{k}$ of isospin $T_{k}$, hypercharge $Y_{k}$ and VEV $v_{k}$ will contribute to $\rho$ as

$$
\rho=\frac{\sum_{k}\left[T_{k}\left(T_{k}+1\right)-\frac{1}{4} Y_{k}^{2}\right] v_{k}^{2} c_{k}}{\sum_{k} \frac{1}{2} Y_{k}^{2} v_{k}^{2}}
$$

where $c_{k}=1 / 2$ (1) for a real (complex) multiplet. Using the formula 4.24) we have

- $\rho=1$ if we only have the SM doublet $\Phi$.

- $\rho=1 / 2$ if we only have the Higgs triplet $\tilde{\chi}$.

- $\rho=\frac{v_{2}^{2}+2 v_{M}^{2}}{v_{2}^{2}+4 v_{M}^{2}}$ if we have both $\Phi$ and $\tilde{\chi}$. Apparently one would have $\rho \neq 1$ when a doublet and a triplet are present.

In order to restore Custodial global SU(2) symmetry $(\rho=1)$ at tree level [66], we add

$$
\xi=(3, Y / 2=0)
$$

and group it with $\tilde{\chi}=(3, Y / 2=1)$ in 6569

$$
\chi=\left(\begin{array}{ccc}
\chi^{0} & \xi^{+} & \chi^{++} \\
\chi^{-} & \xi^{0} & \xi^{+} \\
\chi^{--} & \xi^{-} & \chi^{0 *}
\end{array}\right)
$$

The doublet Higgs $\Phi$ and $\tilde{\Phi}$ can also be grouped in a $2 \times 2$ matrix representation as

$$
\Phi_{2}=\left(\begin{array}{cc}
\phi^{0 *} & \phi^{+} \\
\phi^{-} & \phi^{0}
\end{array}\right)
$$

It we set $\left\langle\chi^{0}\right\rangle=\left\langle\xi^{0}\right\rangle=v_{M}$ and refer to the $\operatorname{VEV}\left\langle\phi^{0}\right\rangle=v_{2} / \sqrt{2}$ we can write the VEVs of $\chi$ and $\Phi_{2}$

$$
\langle\chi\rangle=\left(\begin{array}{ccc}
v_{M} & 0 & 0 \\
0 & v_{M} & 0 \\
0 & 0 & v_{M}
\end{array}\right), \quad\left\langle\Phi_{2}\right\rangle=\left(\begin{array}{cc}
\frac{v_{2}}{\sqrt{2}} & 0 \\
0 & \frac{v_{2}}{\sqrt{2}}
\end{array}\right)
$$


In fact, with $v=\sqrt{v_{2}^{2}+8 v_{M}^{2}}=\Lambda_{E W} \approx 246 \mathrm{GeV}$ we have $M_{W}=g v / 2$ and $M_{Z}=M_{W} / \cos \theta_{W}$. With this proper vacuum alignment $S U(2)_{L} \times U(1)_{Y}$ is explicitly broken down to $U(1)_{e m}$ and the global $S U(2)_{D}$ custodial symmetry is preserved.

This completes the scalar sector in this minimal EW- $\nu_{R}$ model. Before moving on to discuss the possible signature of the model, let us give a brief summary of the particle content as follows

Leptons and quarks (generic notations):

- Doublets

* SM: $l_{L}=\left(\begin{array}{c}\nu_{L} \\ e_{L}\end{array}\right) ; q_{L}=\left(\begin{array}{c}u_{L} \\ d_{L}\end{array}\right)$

* Mirror: $l_{R}^{M}=\left(\begin{array}{c}\nu_{R}^{M} \\ e_{R}^{M}\end{array}\right) ; q_{R}^{M}=\left(\begin{array}{c}u_{R}^{M} \\ d_{R}^{M}\end{array}\right)$

- Singlets

* SM: $e_{R} ; u_{R}, d_{R}$

* Mirror: $e_{L}^{M} ; u_{L}^{M}, d_{L}^{M}$

\section{Higgs fields:}

- A singlet scalar Higgs $\phi_{S}$ with $\left\langle\phi_{S}\right\rangle=v_{S}$.

- Doublet Higgs:

$$
\Phi=\left(\begin{array}{c}
\phi^{+} \\
\phi^{0}
\end{array}\right) \text { with }\left\langle\phi^{0}\right\rangle=v_{2} / \sqrt{2}
$$

- Higgs triplets

$$
* \tilde{\chi}(Y / 2=1)=\frac{1}{\sqrt{2}} \vec{\tau} \cdot \vec{\chi}=\left(\begin{array}{cc}
\frac{1}{\sqrt{2}} \chi^{+} & \chi^{++} \\
\chi^{0} & -\frac{1}{\sqrt{2}} \chi^{+}
\end{array}\right) \text {with }\left\langle\chi^{0}\right\rangle=v_{M} .
$$


$* \xi(Y / 2=0)$ in order to restore Custodial Symmetry with $\left\langle\xi^{0}\right\rangle=v_{M}$.

* VEVs:

$$
v_{2}^{2}+8 v_{M}^{2}=v^{2} \approx(246 \mathrm{GeV})^{2}
$$

In the original version 70, this Higgs doublet couples to both SM and mirror fermions. An extended version was proposed [71] in order to accommodate the 125-GeV SM-like scalar and, in this version, $\Phi_{2}$ only couples to SM fermions while another doublet called $\Phi_{2 M}$ whose $\operatorname{VEV}$ is $\left\langle\phi_{2 M}^{0}\right\rangle=v_{2 M} / \sqrt{2}$ couples only to mirror fermions. Since this thesis focuses only on SM fermions, we will concentrate only on $\Phi_{2}$.

We are now ready to explore the possibility of a direct detection of the right-handed Majorana neutrino of $\mathcal{O}\left(\Lambda_{E W}\right)$ at the colliders. Based upon the fact that $\nu_{R}$ can couple to the $W$ and $Z$ bosons and both $\nu_{R}$ and $e_{R}^{M}$ can decay to $\nu_{L}$ and $e_{L}$ respectively through the scalar singlet $\phi_{S}$. Since the $\nu_{R}$ is a Majorana neutrino type, the main signal could be the same-sign dilepton events which is expressed as

$$
e_{R}^{M,-}+W^{+}+e_{R}^{M,-}+W^{+} \rightarrow e_{L}^{-}+e_{L}^{-}+W^{+}+W^{+}+2 \phi_{S}
$$

It is worthwhile to emphasize that this kind of events is very distinctive in comparison with that of the Dirac neutrino type.

\subsection{Phenomenological constraints}

We will now review several phenomenological constraints of the EW $\nu_{R}$ model as discussed in 71 and 73 . In this review section, we will discuss two sets of results for the EW $\nu_{R}$ model obtained in [71] (the electroweak precision constraints) and 73 (constraints from the 125-GeV SM-like scalar). 


\subsubsection{Electroweak precision constraints on the EW $\nu_{R}$ model 71}

The presence of mirror quark and lepton $S U(2)$-doublets can, by themselves, seriously affect the constraints coming from electroweak precision data. As noticed in [70], the positive contribution to the S-parameter coming from the extra right-handed mirror quark and lepton doublets could be partially cancelled by the negative contribution coming from the triplet Higgs fields. Ref. 71 has carried out a detailed analysis of the electroweak precision parameters $\mathrm{S}$ and $\mathrm{T}$ and found that there is a large parameter space in the model which satisfies the present constraints and that there is no fine tuning due to the large size of the allowed parameter space. It is beyond the scope of the thesis to show more details here but a representative plot would be helpful. Fig. 4.1 shows the contribution of the scalar sector versus that of the mirror fermions to the S-parameter within $1 \sigma$ and $2 \sigma$. In the above plot, 71 took for illustrative purpose 3500 data points that fall inside the $2 \sigma$ region with about 100 points falling inside the $1 \sigma$ region. More details can be found in $[71$.

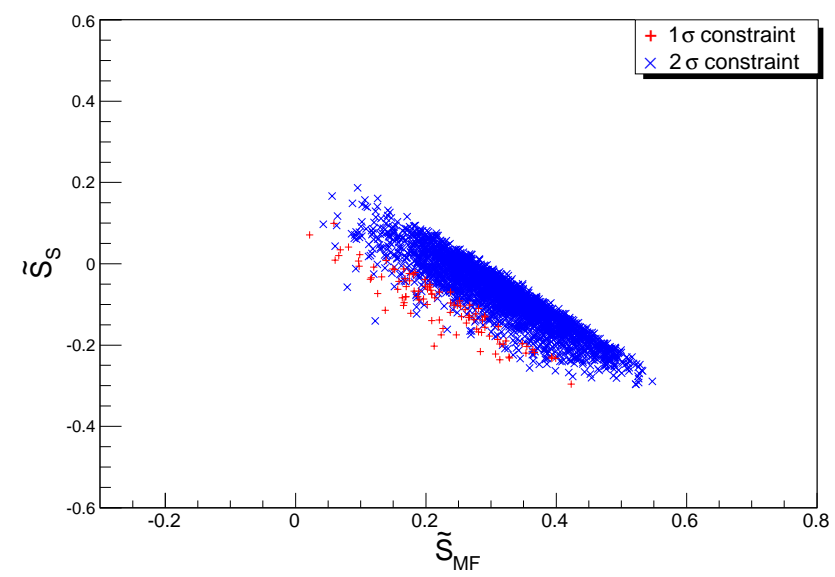

Figure 4.1: Constrained $\tilde{S}_{S}$ versus $\tilde{S}_{M F}$ 


\subsubsection{Review of the scalar sector of the EW $\nu_{R}$ model in light of the discovery of the 125-GeV SM-like scalar 73}

In light of the discovery of the $125-\mathrm{GeV}$ SM-like scalar, it is imperative that any model beyond the SM (BSM) shows a scalar spectrum that contains at least one Higgs field with the desired properties as required by experiment. The present data from CMS and ATLAS only show signal strengths that are compatible with the SM Higgs boson. The definition of a signal strength $\mu$ is as follows

$$
\sigma(H \text {-decay })=\sigma(H \text {-production }) \times B R(H \text {-decay })
$$

and

$$
\mu(H \text {-decay })=\frac{\sigma(H \text {-decay })}{\sigma_{S M}(H \text {-decay })} .
$$

To really distinguish the SM Higgs field from its impostor, it is necessary to measure the partial decay widths and the various branching ratios. In the present absence of such quantities, the best one can do is to present cases which are consistent with the experimental signal strengths. This is what was carried out in 73$]$.

The minimization of the potential containing the scalars shown above breaks its global symmetry $S U(2)_{L} \times S U\left(20_{R}\right.$ down to a custodial symmetry $S U(2)_{D}$ which guarantees at tree level $\rho=M_{W}^{2} / M_{Z}^{2} \cos ^{2} \theta_{W}=173$. The physical scalars can be grouped, based on their transformation properties under $S U(2)_{D}$ as follows:

$$
\begin{aligned}
\text { five-plet (quintet) } & \rightarrow H_{5}^{ \pm \pm}, H_{5}^{ \pm}, H_{5}^{0} ; \\
\text { triplet } & \rightarrow H_{3}^{ \pm}, H_{3}^{0} ; \\
\text { triplet } & \rightarrow H_{3 M}^{ \pm}, H_{3 M}^{0} ; \\
\text { three singlets } & \rightarrow H_{1}^{0}, H_{1 M}^{0}, H_{1}^{0 \prime},
\end{aligned}
$$

The three custodial singlets are the CP-even states, one combination of which can be the $125-\mathrm{GeV}$ 
scalar. In terms of the original fields, one has

$$
\begin{aligned}
H_{1}^{0} & =\phi_{2}^{0 r}, \\
H_{1 M}^{0} & =\phi_{2 M}^{0 r}, \\
H_{1}^{0 \prime} & =\frac{1}{\sqrt{3}}\left(\sqrt{2} \chi^{0 r}+\xi^{0}\right) .
\end{aligned}
$$

These states mix through a mass matrix obtained from the potential and the mass eigenstates are

denoted by $\widetilde{H}, \widetilde{H}^{\prime}$, and $\widetilde{H}^{\prime \prime}$, with the convention that the lightest of the three is denoted by $\widetilde{H}$, the next heavier one by $\widetilde{H}^{\prime}$ and the heaviest state by $\widetilde{H}^{\prime \prime}$.

To compute the signal strengths $\mu$, Ref. 73 considers $\widetilde{H} \rightarrow Z Z, W^{+} W^{-}, \gamma \gamma, b \bar{b}, \tau \bar{\tau}$. In addition, the cross section of $g g \rightarrow \widetilde{H}$ related to $\widetilde{H} \rightarrow g g$ was also calculated. A scan over the parameter space of the model yielded two interesting scenarios for the $125-\mathrm{GeV}$ scalar:

(1) Dr Jekyll's scenario in which $\widetilde{H} \sim H_{1}^{0}$ meaning that the SM-like component $H_{1}^{0}=\phi_{2}^{0 r}$ is dominant.

(2) Mr Hyde's scenario in which $\widetilde{H} \sim H_{1}^{0 \prime}$ meaning that the SM-like component $H_{1}^{0}=\phi_{2}^{0 r}$ is subdominant.

Both scenarios give signal strengths compatible with experimental data as shown below in Fig. 4.2. As we can see from Fig. (4.2), both SM-like scenario (Dr Jekyll) and the more interesting scenario which is very unlike the SM (Mr Hyde) agree with experiment. As stressed in [73, present data cannot tell whether or not the $125-\mathrm{GeV}$ scalar is truly SM-like or even if it has a dominant SM-like component. It has also been stressed in 73 that it is essential to measure the partial decay widths of the $125-\mathrm{GeV}$ scalar to truly reveal its nature. Last but not least, in both scenarios, $H_{1 M}^{0}=\phi_{2 M}^{0 r}$ is subdominant but is essential to obtain the agreement with the data as shown in 73 .

As discussed in detail in 73, for proper vacuum alignment, the potential contains a term 


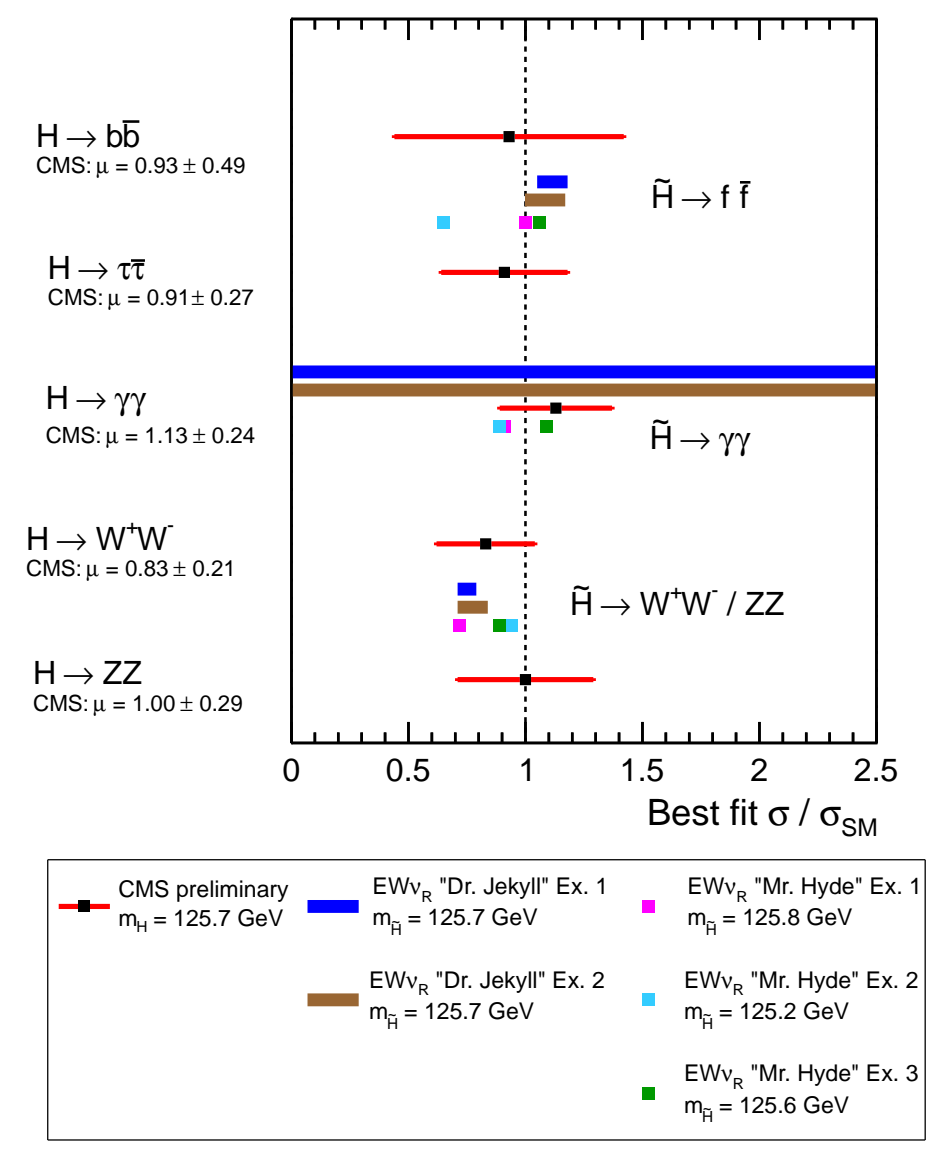

Figure 4.2: Figure shows the predictions of $\mu\left(\widetilde{H} \rightarrow b \bar{b}, \tau \bar{\tau}, \gamma \gamma, W^{+} W^{-}, Z Z\right)$ in the EW $\nu_{R}$ model for examples 1 and 2 in Dr. Jekyll and example 1, 2 and 3 in Mr. Hyde scenarios as discussed in [73, in comparison with corresponding best fit values by CMS 75,78$]$.

proportional to $\lambda_{5}$ (Eq. (32) of [73]) and it is this term that prevents the appearance of NambuGoldstone (NG) bosons in the model. The would-be NG bosons acquire a mass proportional to $\lambda_{5}$. An analysis of CP-odd scalar states $H_{3}^{0}, H_{3 M}^{0}$ and the heavy CP-even states $\widetilde{H}^{\prime}$, and $\widetilde{H}^{\prime \prime}$ was presented in 73$]$. The phenomenology of charged scalars including the doubly-charged ones was also discussed in 65.

The phenomenology of mirror quarks and leptons was briefly discussed in 70 and a detailed analysis of mirror quarks was presented in [74]. It suffices to mention here that mirror fermions decay into SM fermions through the process $q^{M} \rightarrow q \phi_{S}, l^{M} \rightarrow l \phi_{S}$ with $\phi_{S}$ "appearing" as missing energy in the detector. Furthermore, the decay of mirror fermions into SM ones can happen outside 
the beam pipe and inside the silicon vertex detector. Searches for non-SM fermions do not apply in this case. It is beyond the scope of the thesis to discuss these details here.

This concludes the brief summary of the EW $\nu_{R}$ model [70]. The original minimal model contains just one singlet Higgs field $\phi_{S}$. As we shall see in the next chapter, we will present a different model which will incorporates in the $A_{4}$ symmetry and necessitate an extension to four Higgs singlet fields with no phenomenological constraints at the present time. 


\section{Chapter 5}

\section{Model of Neutrino Masses and Mixings}

The discovery and subsequent analyses of neutrino oscillation phenomena have revealed a trove of valuable information concerning the mixing matrix $U_{P M N S}$ and the mass difference squared in the neutrino sector. It is worth stressing that the mere presence of neutrino masses as implied by the oscillation data [79] provides the first evidence of physics beyond the Standard Model (BSM). What might be the origin of neutrino masses? Why are they so tiny $\left(m_{\nu}<O(e V)\right)$ as compared with even that of the lightest of elementary particles: the electron? Why is the leptonic mixing matrix $U_{P M N S}$ so different from $V_{C K M}$ of the quark sector? Is there any chance that some of the physics that are responsible for the tininess of the neutrino masses as well as their mixings could somehow be experimentally accessible at the Large Hadron Collider (LHC) in the near future or even at the International Linear Collider at a not-too-distant future?

The vast difference between neutrino masses and those of other elementary particles is a big mystery. There could be several ways in which neutrinos can obtain masses, all of which go beyond the Standard Model. The most obvious one is to add right-handed neutrinos which are singlets of the SM and couple them through Yukawa interactions with the left-handed lepton doublets and the SM Higgs doublet. In this simplest Dirac mass scenario, the Yukawa coupling would have to be unnaturally small i.e. $g_{\nu} \lesssim O\left(10^{-11}\right)$ in order to accommodate $m_{\nu}<O(e V)$. A more elegant scenario is the quintessential see-saw mechanism [80] where the right-handed neutrinos 
acquire a Majorana mass term $M_{R} \nu_{R}^{T} \sigma_{2} \nu_{R}$ in addition to a Dirac mass term $m_{D} \nu_{L}^{\dagger} \nu_{R}+$ h.c.. The diagonalization of the mass matrix yields two eigenvalues whose magnitudes are approximately $m_{D}^{2} / M_{R}$ and $M_{R}$ for $M_{R} \gg m_{D}$. In a generic see-saw scenario, right-handed neutrinos are SM singlets (e.g. in an $\mathrm{SO}(10)$ scenario) i.e. they are sterile, and typically $m_{D} \propto O\left(\Lambda_{E W}\right)$ and $M_{R} \propto O\left(\Lambda_{G U T}\right)$ or $O\left(M_{W_{R}}\right)$. Although this generic scenario can elegantly "explain" the smallness of neutrino masses, it goes without saying that the prospect of directly testing the seesaw mechanism by searching for right-handed neutrinos is very remote, both from an energetic point of view and from a production point of view. (Although it is very popular, leptogenesis by itself is not such a direct test.)

P.Q. Hung has proposed a model [70] of electroweak-scale right-handed neutrinos in which (as we will briefly review below) $\nu_{R}$ 's belong to $S U(2)$ doublets along with mirror charged leptons. This has two distinct advantages: 1) $\nu_{R}$ 's are non-sterile and couple to the $\mathrm{Z}$ and $\mathrm{W}$ bosons; 2) Since $\nu_{R}$ 's are members of doublets, a Majorana mass term necessarily comes from the vacuum expectation value $(\mathrm{VEV})$ of a triplet Higgs field which spontaneously breaks $S U(2) \times U(1)_{Y}$ (in addition to the Higgs doublet) and, as a result, $M_{R} \propto O\left(\Lambda_{E W}\right)$. In this scenario, the EW $\nu_{R}$ model, right-handed neutrinos can be produced and searched for at the LHC or at the proposed ILC.

On the scalar sector side, the minimal EW $\nu_{R}$ model contains one Higgs doublet, one complex triplet, one real triplet and one Higgs singlet. The role of these Higgs fields in generating fermion masses will be discussed below. In particular, we will discuss the importance of the Higgs singlet on the issue of neutrino masses.

The plan of this chapter will be as follows. First we discuss the motivation for using the nonAbelian discrete symmetry $A_{4}[81$ to describe the Dirac part of the neutrino mass matrix which, in the EW $\nu_{R}$ model, is generated by the Higgs singlets. In this case, we increase the number of Higgs singlets from one (the number in the original model) to four without any consequence as far as the $125-\mathrm{GeV}$ SM-like Higgs boson is concerned. The number of non-singlet Higgs fields is kept 
unchanged in view of the tight constraints coming from the properties of the $125-\mathrm{GeV}$ object as discussed in [73]. Secondly, we give a possibility to explain the difference between two well-known PMNS and CKM matrices. We then move toward the charged lepton sector to explore some of interesting features within the framework of this model of neutrino masses and mixings.

\subsection{Motivation}

It is a big puzzle why the quark mixing matrix, the so-called CKM matrix [82]

$$
\left|V_{C K M}\right|=\left(\begin{array}{ccc}
0.9743 \pm 0.0002 & 0.2255 \pm 0.0024 & (5.10 \pm 0.47) \times 10^{-3} \\
0.230 \pm 0.011 & 1.006 \pm 0.023 & (40.9 \pm 1.1) \times 10^{-3} \\
(8.4 \pm 0.6) \times 10^{-3} & (42.9 \pm 2.6) \times 10^{-3} & 0.89 \pm 0.07
\end{array}\right)
$$

(which is not too different from the unit matrix) differs so much from the leptonic one, the so-called Pontecorvo-Maki-Nakagawa-Sakata (PMNS) matrix 82

$$
\left|U_{P M N S}\right|=\left(\begin{array}{ccc}
0.779 \ldots 0.848 & 0.510 \ldots 0.604 & 0.122 \ldots 0.190 \\
0.183 \ldots 0.568 & 0.385 \ldots 0.728 & 0.613 \ldots 0.794 \\
0.200 \ldots 0.576 & 0.408 \ldots 0.742 & 0.589 \ldots 0.775
\end{array}\right) \text {. }
$$

Although the precise mass mechanism is far from being understood, it is not too unreasonable to speculate that the aforementioned big difference arises from the way neutrinos obtain masses as compared with the way charged fermions obtain theirs. In this section, we will present a model in which it is the Dirac mass matrix of the neutrinos that is obtained by incorporating an $A_{4}$ symmetry into the model. Before taking more steps to explore the model of neutrino masses and mixings, let us briefly review the $A_{4}$ symmetry.

\section{- An introduction to $A_{4}$ symmetry}

The $A_{4}$ group is a non-Abelian discrete group [81] and also known as the group of rotational symmetry of a tetrahedron. This is a finite group of 12 permutations (or symmetries) of four 
elements, which can be organized in four classes whose number of elements are 1, 3, 4, 4. This implies four irreducible representations of dimensions $n_{k}$ satisfying the condition

$$
\sum_{k} n_{k}^{2}=12
$$

In this case, the only possible solutions for 5.3 are

$$
n_{1}=n_{2}=n_{3}=1 \text { and } n_{4}=3
$$

i.e. there are three $1-\mathrm{D}$ and one $3-\mathrm{D}$ representations of $A_{4}$. We shall denote them as $\underline{1}, \underline{1}^{\prime}, \underline{1}^{\prime \prime}$, and 3. These representations obey the product rule given by (using the notation of [83]):

$$
\begin{aligned}
\underline{3} \times \underline{3}= & \underline{1}(11+22+33)+\underline{1}^{\prime}\left(11+\omega^{2} 22+\omega 33\right)+\underline{1}^{\prime \prime}\left(11+\omega 22+\omega^{2} 33\right) \\
& +\underline{3}(23,31,12)+\underline{3}(32,13,21) .
\end{aligned}
$$

Here $\omega=e^{i 2 \pi / 3}$, called the cube root of unity.

\section{- $A_{4}$ model-wide recognitions}

The $A_{4}$ symmetry is well-known not only in group theory but also in model buildings. Which particles are assigned to which representations of $A_{4}$ is a question which depends entirely on the model one is dealing with. Below we briefly summarize two popular $A_{4}$ models in order to show the contrasts with ours.

$A_{4}$ has widely been used to produce the tribimaximal form of the PMNS matrix [83]. In a nutshell, as summarized nicely in [83, the $A_{4}$ symmetry is usually applied to the charged lepton mass matrix with the result being that the unitary matrix which diagonalizes the charged lepton mass matrix takes on the form of the PMNS that was first proposed by Cabibbo and Wolfenstein [84, namely

$$
U_{C W}=\frac{1}{\sqrt{3}}\left(\begin{array}{ccc}
1 & 1 & 1 \\
1 & \omega & \omega^{2} \\
1 & \omega^{2} & \omega
\end{array}\right)
$$


In popular versions of $A_{4}$-inspired models of neutrino mass and mixing, $U_{C W}$ is identified with the unitary matrix $U_{l L}$ which diagonalizes the charged lepton mass matrix.

In one version [83, the left-handed lepton doublets are assigned to $\underline{3}$ while the right-handed $S U(2)$-singlet charged leptons are assigned to $\underline{1}, \underline{1}^{\prime}, \underline{1}^{\prime \prime}$. There are three Higgs doublets belonging to 3. In another version, there are 4 Higgs doublets and the right-handed charged leptons belong to a $\underline{3}$. As described in 83 , the main feature of these models is the diagonalization of the charged lepton mass matrix by $U_{C W}$ in Eq. (5.6) and the presence of three or four Higgs doublets. We shall make some remarks concerning the implication of the 125-GeV SM-like boson on models with extended Higgs sectors.

In [85], a supersymmetric model was written which now includes three families of $S U(2)$ singlet vector-like heavy quarks and leptons, two Higgs doublets transforming as $\underline{1}$ and three Higgs singlets that transform as $\underline{3}$ of $A_{4}$. Here the SM right-handed fermions transform like $\underline{1}, \underline{1}^{\prime}, \underline{1}^{\prime \prime}$. Just as with the models mentioned above 83 , the construction is such that $U_{C W}$ in Eq. (5.6) is the matrix which diagonalizes the charged lepton mass matrix.

The two examples discussed above are two of several scenarios making use of the $A_{4}$ symmetry. It is beyond the scope of this thesis to compare our approach with all others that are present in the literature. The main point we would like to stress here is that the most popular scenario is one in which the $A_{4}$ symmetry is used to generate $U_{C W}$ in Eq. (5.6) for the charged lepton sector. Also, right-handed neutrinos in most generic models are SM-singlets and their Majorana masses are expected to be much larger than the EW scale.

Before discussing our approach based on $A_{4}$, we would like to point out the main differences with the aforementioned scenarios: 1) The conjugate of the matrix as shown in Eq. (5.6) is the one that diagonalizes the neutrino Dirac mass matrix; 2) Right-handed neutrinos belong to $S U(2)$ doublets along with mirror charged leptons as espoused in 70 and are therefore non-sterile. Their 
Majorana masses are proportional to the EW symmetry breaking scale.

\subsection{Assignment of particle content}

As the most mysterious particles in the world neutrinos have been studied for years by many physicists. The fact that neutrinos have masses motivates particle theorists to "create" an adequate model which leads them to a clear understanding of neutrino masses. The EW- $\nu_{R}$ model combing with the $A_{4}$ symmetry is a new approach to our big goal because it introduces three new scalar Higgs singlets $\phi_{1 S}, \phi_{2 S}, \phi_{3 S}$ without conflicting with the LHC data which severely constrained the Higgs doublet sector. Moreover, the reason which makes the $A_{4}$ symmetry become a good candidate for this task is its simplicity and sufficiency.

Let us now present the assignments of the EW $\nu_{R}$ model's content under $A_{4}$ as shown in Table (5.1).

Table 5.1: $A_{4}$ assignments for leptons and Higgs fields

\begin{tabular}{|c||c|c|c|c|c|c|c|}
\hline Field & $(\nu, l)_{L}$ & $\left(\nu, l^{M}\right)_{R}$ & $e_{R}$ & $e_{L}^{M}$ & $\phi_{0 S}$ & $\tilde{\phi}_{S}=\left(\begin{array}{c}\phi_{1 S} \\
\phi_{2 S} \\
\phi_{3 S}\end{array}\right)$ & $\Phi_{2}$ \\
\hline$A_{4}$ & $\underline{3}$ & $\underline{3}$ & $\underline{3}$ & $\underline{3}$ & $\underline{1}$ & $\underline{3}$ & $\underline{1}$ \\
\hline
\end{tabular}

Notice that if one had the singlet Higgs fields belonged to $\underline{1}, \underline{1}^{\prime}$, and $\underline{1}^{\prime \prime}$ only, the neutrino Dirac mass matrix would be diagonal which is not a desired scenario. 


\subsection{Neutrino Dirac and Majorana masses with $A_{4}$ symmetry}

\subsubsection{Neutrino Dirac mass matrix}

As shown in [70], the neutrino Dirac mass in the EW $\nu_{R}$ model comes from the generic Yukawa term $g_{S l} \bar{l}_{L} \phi_{S} l_{R}^{M}+$ h.c. in Eq. 4.8). With the $A_{4}$ assignments shown in Table (5.1), we can write the following Yukawa interactions

$$
L_{S}=\bar{l}_{L}\left(g_{0 S} \phi_{0 S}+g_{1 S} \tilde{\phi}_{S}+g_{2 S} \tilde{\phi}_{S}\right) l_{R}^{M}+\text { h.c. },
$$

where $g_{1 S}$ and $g_{2 S}$ reflect the two different ways that $\tilde{\phi}_{S}$ couples to the product of $\bar{l}_{L}$ and $l_{R}^{M}$ as shown in Eq. (5.5). We obtain the following neutrino Dirac mass matrix:

$$
M_{\nu}^{D}=\left(\begin{array}{ccc}
g_{0 S} v_{0} & g_{1 S} v_{3} & g_{2 S} v_{2} \\
g_{2 S} v_{3} & g_{0 S} v_{0} & g_{1 S} v_{1} \\
g_{1 S} v_{2} & g_{2 S} v_{1} & g_{0 S} v_{0}
\end{array}\right)
$$

where $v_{0}=\left\langle\phi_{0 S}\right\rangle$ and $v_{i}=\left\langle\phi_{i S}\right\rangle$ with $\imath=1,2,3$. Notice that this form of $M_{\nu}^{D}$ is the same as the one used by [83] for the charged lepton mass matrix.

When $v_{1}=v_{2}=v_{3}=v, M_{\nu}^{D}$ can be diagonalized as follows (using $1+\omega+\omega^{2}=0$ and $\left.\omega^{2}=\omega^{*}\right)$

$$
U_{\nu}^{\dagger} M_{\nu}^{D} U_{\nu}=\left(\begin{array}{ccc}
m_{1 D} & 0 & 0 \\
0 & m_{2 D} & 0 \\
0 & 0 & m_{3 D}
\end{array}\right)
$$

where

$$
U_{\nu}=\frac{1}{\sqrt{3}}\left(\begin{array}{ccc}
1 & 1 & 1 \\
1 & \omega^{2} & \omega \\
1 & \omega & \omega^{2}
\end{array}\right)
$$

Notice that our $U_{\nu}$ defined in Eq. 5.10 is just $U_{\nu}=U_{C W}^{\dagger}$. At this point, we would like to establish our notations for what will follow. In general, a mass matrix is diagonalized by two unitary matrices 
$U_{L}$ and $U_{R}$ i.e.

$$
U_{L}^{\dagger} \mathcal{M} U_{R}=\mathcal{M}_{D}
$$

where $M_{D}$ is a diagonal mass matrix. A mass term of the form $\bar{f}_{L}^{0} \mathcal{M} f_{R}^{0}$ can be rewritten as $\bar{f}_{L}^{0} U_{L} U_{L}^{\dagger} \mathcal{M} U_{R} U_{R}^{\dagger} f_{R}^{0}=\bar{f}_{L} \mathcal{M}_{D} f_{R}$ where $\bar{f}_{L}^{0} U_{L}=\bar{f}_{L}$ and $U_{R}^{\dagger} f_{R}^{0}=f_{R}$

From Eq. (5.9), it is clear that

$$
U_{\nu L}=U_{\nu R}=U_{\nu}
$$

A remark is in order at this point. As we will see below, $U_{P M N S}$ is defined as $U_{P M N S}=U_{\nu L}^{\dagger} U_{l L}=$ $U_{\nu}^{\dagger} U_{l L}$. What $U_{l L}$ might be will be the subject of the section on the charged lepton mass matrix.

The neutrino Dirac masses are

$$
\begin{aligned}
& m_{1 D}=g_{0 S} v_{0}+g_{1 S} v+g_{2 S} v \\
& m_{2 D}=g_{0 S} v_{0}+g_{1 S} v \omega^{2}+g_{2 S} v \omega \\
& m_{3 D}=g_{0 S} v_{0}+g_{1 S} v \omega+g_{2 S} v \omega^{2}
\end{aligned}
$$

Reality of the masses require that

$$
g_{2 S}=g_{1 S}^{*},
$$

where we have used $\omega^{2}=\omega^{*}$. Making use of $1+\omega+\omega^{2}=0, \omega^{3}=1$ and Eq. (5.16), we obtain the following sum rules

$$
\begin{aligned}
& m_{1 D}+m_{2 D}+m_{3 D}=3 g_{0 S} v_{0} \\
& m_{1 D}^{2}+m_{2 D}^{2}+m_{3 D}^{2}=3 g_{0 S}^{2} v_{0}^{2}+6\left|g_{1 S}\right|^{2} v^{2} .
\end{aligned}
$$

\subsubsection{Neutrino Majorana mass matrix}

From the Lagrangian

$$
L_{M}=g_{M}\left(l_{i R}^{M, T} \sigma_{2}\right)\left(i \tau_{2} \tilde{\chi}\right) l_{j R}^{M}+h . c .
$$


In order to make the Lagrangian invariant under $A_{4}$, we need $\tilde{\chi}$ to transform as $\underline{1}$ or $\underline{3}$. For reasons outlined in 73 having to do with the constraints coming from the presently known properties of the $125-\mathrm{GeV}$ SM-like boson, it is preferable that the Higgs triplet transforms as $\underline{1}$. We recall that

$$
\tilde{\chi}=\frac{1}{\sqrt{2}} \vec{\tau} \cdot \vec{\chi}=\left(\begin{array}{cc}
\frac{1}{\sqrt{2}} \chi^{+} & \chi^{++} \\
\chi^{0} & -\frac{1}{\sqrt{2}} \chi+
\end{array}\right)
$$

When $\left\langle\chi^{0}\right\rangle=v_{M}$ one obtains the following right-handed Majorana mass

$$
M_{R}=\left(\begin{array}{ccc}
g_{M}\left\langle\chi^{0}\right\rangle & 0 & 0 \\
0 & g_{M}\left\langle\chi^{0}\right\rangle & 0 \\
0 & 0 & g_{M}\left\langle\chi^{0}\right\rangle
\end{array}\right)=g_{M} v_{M} \mathbb{I}
$$

Therefore, the neutrino mass matrix is

$$
M_{\nu}=\left(\begin{array}{cc}
0 & M_{\nu}^{D} \\
M_{\nu}^{D} & M_{R}
\end{array}\right)
$$

Here the $3 \times 3$ see-saw mass matrix for the light neutrinos $\left(\nu_{e}, \nu_{\mu}, \nu_{\tau}\right)$ becomes

$$
m_{\nu} \sim-M_{\nu}^{D} M_{R}^{-1} M_{\nu}^{D, T}
$$

\section{$5.4 U_{P M N S}$ vs. $V_{C K M}$}

\subsubsection{The search for $U_{l L}$}

As mentioned above, we define the diagonalization of a mass matrix by Eq. (5.11). The charged current interaction $g \bar{\nu}_{L}^{0} \gamma^{\mu} l_{L}^{0} W_{\mu}^{+}$can be written in terms of mass eigenstates as

$$
g \bar{\nu}_{L}^{0} U_{\nu L} U_{\nu L}^{\dagger} \gamma^{\mu} U_{l L} U_{l L}^{\dagger} l_{L}^{0} W_{\mu}^{+}=g \bar{\nu}_{L} U_{P M N S} \gamma^{\mu} l_{L} W_{\mu}^{+}
$$


where $\nu_{L}$ and $l_{L}$ are mass eigenstates and where

$$
U_{P M N S}=U_{\nu L}^{\dagger} U_{l L}=U_{\nu}^{\dagger} U_{l L}
$$

Notice that, by looking at $U_{P M N S}$ as determined from experimental data in Eq. (5.2), one can safely say that $U_{P M N S} \neq U_{\nu}^{\dagger}$. One needs $U_{l L}$ to be different from the unit matrix. But could $U_{l L}$ be? What does the Yukawa coupling of the charged leptons to $\Phi_{2}$ tell us about $U_{l L}$ ? (There is a coupling between the mirror and SM charged leptons with the Higgs singlets but its contributions to the masses are negligible as shown in 70$]$. We will ignore this contribution here.)

The SM Yukawa coupling is

$$
L_{Y}=g_{l} \bar{l}_{L} \Phi_{2} e_{R}+\text { h.c. }
$$

where $\Phi_{2}=\left(\begin{array}{c}\phi^{+} \\ \phi^{0}\end{array}\right),\left\langle\phi^{0}\right\rangle=\frac{v_{2}}{\sqrt{2}}$

From Table (5.1), we have the following $A_{4}$ assignments:

$$
l_{L} \sim \underline{3} ; \quad e_{R} \sim \underline{3} ; \quad \Phi_{2} \sim \underline{1} .
$$

It can be seen that (5.26) is $A_{4}$-invariant. From the product rule (5.5), one can see that this $A_{4}$-invariant Yukawa term gives a degenerate spectrum for the charged leptons, namely

$$
\mathcal{M}_{l}=g_{l} \frac{v_{2}}{\sqrt{2}}\left(\begin{array}{ccc}
1 & 0 & 0 \\
0 & 1 & 0 \\
0 & 0 & 1
\end{array}\right)
$$

Diagonalizing the matrix $\mathcal{M}_{l}$ by $U_{l L}^{\dagger} \mathcal{M}_{l} U_{l R}$ would imply that $U_{l L}=\mathbb{I}$.

If this were the whole story, one could have

$$
U_{P M N S}=U_{\nu}^{\dagger}=\frac{1}{\sqrt{3}}\left(\begin{array}{ccc}
1 & 1 & 1 \\
1 & \omega & \omega^{2} \\
1 & \omega^{2} & \omega
\end{array}\right) .
$$


This implies that the PMNS matrix mainly comes from neutrino mixing matrix which results from the couplings between leptons and the Higgs singlet $\phi_{S}$. On the other hand, as reviewed in section 2.4, we have acknowledged that

$$
V_{C K M}=U_{u, L}^{\dagger} U_{d, L}
$$

This mixing matrix comes totally from Yukawa couplings of quarks to the SM Higgs doublet $\Phi$. Therefore, in our analysis, the different sources of mixing matrices provide a reasonable explanation for the intrinsic difference between the PMNS and $C K M$ matrices.

However the story does not end here. The fact that $U_{l L}$ is a unit matrix is unacceptable for two reasons: 1) $m_{e} \ll m_{\mu}<m_{\tau}$;2) $U_{l L}$ would be a unit matrix and one would obtain $U_{P M N S}=U_{\nu L}^{\dagger}$ in disagreement with experiment. It is then clear that the $A_{4}$ symmetry which is respected by the Yukawa interactions in Eq. 6.1 giving rise to the neutrino Dirac mass matrix has to be broken in the charged lepton sector. In what follows, we will use a phenomenological approach toward this $A_{4}$ breaking, namely through an ansätz for $U_{l L}$.

\subsubsection{Ansätz for $U_{l L}$}

As discussed above, strict $A_{4}$ symmetry in the charged lepton sector would imply that $U_{l L}=\mathbb{I}$. We will parametrize the breaking of $A_{4}$ by assuming a form which deviates from the unit matrix by a small amount and which is unitary. Using $U_{P M N S}$ and $U_{\nu}$, one can then determine $U_{l L}$. As we shall see below, once $U_{l L}$ is known, one can reconstruct $\mathcal{M}_{l} \mathcal{M}_{l}^{\dagger}$. In this sense, our approach is semi phenomenological because we do not use a specific symmetry assumption to construct the charged lepton mass matrix. 
We propose the following ansätz

$$
U_{l L}=\left(\begin{array}{ccc}
1-\frac{\lambda_{l}^{2}}{2} & \lambda_{l} & A_{l} \lambda_{l}^{3}\left(\rho_{l}-i \eta_{l}\right) \\
-\lambda_{l} & 1-\frac{\lambda_{l}^{2}}{2} & A_{l} \lambda_{l}^{2} \\
A_{l} \lambda_{l}^{3}\left(1-\rho_{l}-i \eta_{l}\right) & -A_{l} \lambda_{l}^{2} & 1
\end{array}\right)
$$

where $A_{l}, \rho_{l}, \eta_{l}$ are real parameters of $\mathrm{O}(1) 22$. The subscript $l$ indicates that $A, \rho, \eta$ belong to charged leptons.

We can now constrain $\lambda_{l}, A_{l}, \rho_{l}, \eta_{l}$ based on experimental data of $U_{P M N S}$ and unitarity conditions and have

$$
U=U_{P M N S}=U_{\nu}^{\dagger} U_{l L}=\frac{1}{\sqrt{3}}\left(\begin{array}{ccc}
1 & 1 & 1 \\
1 & \omega^{2 *} & \omega^{*} \\
1 & \omega^{*} & \omega^{2 *}
\end{array}\right)\left(\begin{array}{ccc}
1-\frac{\lambda_{l}^{2}}{2} & \lambda_{l} & A_{l} \lambda_{l}^{3}\left(\rho_{l}-i \eta_{l}\right) \\
-\lambda_{l} & 1-\frac{\lambda_{l}^{2}}{2} & A_{l} \lambda_{l}^{2} \\
A_{l} \lambda_{l}^{3}\left(1-\rho_{l}-i \eta_{l}\right) & -A_{l} \lambda_{l}^{2} & 1
\end{array}\right)
$$

Recall that $\omega=e^{i 2 \pi / 3}$ so $\omega^{*}=\omega^{2}$ and $\omega^{2 *}=\omega$. Therefore,

$$
\begin{aligned}
& U=\frac{1}{\sqrt{3}}\left(\begin{array}{ccc}
1 & 1 & 1 \\
1 & \omega & \omega^{2} \\
1 & \omega^{2} & \omega
\end{array}\right)\left(\begin{array}{ccc}
1-\frac{\lambda_{l}^{2}}{2} & \lambda_{l} & A_{l} \lambda_{l}^{3}\left(\rho_{l}-i \eta_{l}\right) \\
-\lambda_{l} & 1-\frac{\lambda_{l}^{2}}{2} & A_{l} \lambda_{l}^{2} \\
A_{l} \lambda_{l}^{3}\left(1-\rho_{l}-i \eta_{l}\right) & -A_{l} \lambda_{l}^{2} & 1
\end{array}\right) \\
& =\frac{1}{\sqrt{3}}\left(\begin{array}{ccc}
A_{l} \lambda_{l}^{3}\left(1-\rho_{l}-i \eta_{l}\right)-\frac{\lambda_{l}^{2}}{2}-\lambda_{l}+1 & -\left(A_{l}+\frac{1}{2}\right) \lambda_{l}^{2}+\lambda_{l}+1 & A_{l} \lambda_{l}^{3}\left(\rho_{l}-i \eta_{l}\right)+A_{l} \lambda_{l}^{2}+1 \\
\omega^{2} A_{l} \lambda_{l}^{3}\left(1-\rho_{l}-i \eta_{l}\right)-\frac{\lambda_{l}^{2}}{2}-\omega \lambda_{l}+1 & -\left(\omega^{2} A_{l}+\frac{\omega}{2}\right) \lambda_{l}^{2}+\lambda_{l}+\omega & A_{l} \lambda_{l}^{3}\left(\rho_{l}-i \eta_{l}\right)+\omega A_{l} \lambda_{l}^{2}+\omega^{2} \\
\omega A_{l} \lambda_{l}^{3}\left(1-\rho_{l}-i \eta_{l}\right)-\frac{\lambda_{l}^{2}}{2}-\omega^{2} \lambda_{l}+1 & -\left(\omega A_{l}+\frac{\omega^{2}}{2}\right) \lambda_{l}^{2}+\lambda_{l}+\omega^{2} & A_{l} \lambda_{l}^{3}\left(\rho_{l}-i \eta_{l}\right)+\omega^{2} A_{l} \lambda_{l}^{2}+\omega
\end{array}\right)
\end{aligned}
$$


Recall the standard parametrization of PMNS matrix ( [86], 87])

$$
\begin{aligned}
& U=V\left(\begin{array}{ccc}
1 & 0 & 0 \\
0 & e^{i \frac{\alpha_{21}}{2}} & 0 \\
0 & 0 & e^{i \frac{\alpha_{31}}{2}}
\end{array}\right) \\
& V=\left(\begin{array}{ccccc}
c_{12} c_{13} & s_{12} c_{13} & s_{13} e^{-i \delta} \\
-s_{12} c_{23}-c_{12} s_{23} s_{13} e^{i \delta} & c_{12} c_{23}-s_{12} s_{23} s_{13} e^{i \delta} & s_{23} c_{13} \\
s_{12} s_{23}-c_{12} c_{23} s_{13} e^{i \delta} & -c_{12} s_{23}-s_{12} c_{23} s_{13} e^{i \delta} & c_{23} c_{13}
\end{array}\right)
\end{aligned}
$$

where $s_{i j} \equiv \sin \left(\theta_{i j}\right), c_{i j} \equiv \cos \left(\theta_{i j}\right), \theta_{i j} \in\left[0, \frac{\pi}{2}\right], \alpha_{21}, \alpha_{31}$ are Majorana phases.

For the purposes of this thesis, the Majorana phases will not be taken into account, i.e. we can set these phases to be equal to zero. Therefore, our PMNS matrix really has the form of V which contains the Dirac phase.

Let us compare Eq. 5.32 with experimental data 82

$$
|U|=\left(\begin{array}{ccc}
0.779 \ldots 0.848 & 0.510 \ldots 0.604 & 0.122 \ldots 0.190 \\
0.183 \ldots 0.568 & 0.385 \ldots 0.728 & 0.613 \ldots 0.794 \\
0.200 \ldots 0.576 & 0.408 \ldots 0.742 & 0.589 \ldots 0.775
\end{array}\right)
$$


we have the following constraints

$$
\begin{aligned}
\text { (i) } \quad 0.779 & <\frac{1}{\sqrt{3}}\left|A_{l} \lambda_{l}^{3}\left(1-\rho_{l}-i \eta_{l}\right)-\frac{\lambda_{l}^{2}}{2}-\lambda_{l}+1\right|<0.848 \\
(\text { ii }) \quad 0.510 & <\frac{1}{\sqrt{3}}\left|-\left(A_{l}+\frac{1}{2}\right) \lambda_{l}^{2}+\lambda_{l}+1\right|<0.604 \\
(\text { iii }) \quad 0.122 & <\frac{1}{\sqrt{3}}\left|A_{l} \lambda_{l}^{3}\left(\rho_{l}-i \eta_{l}\right)+A_{l} \lambda_{l}^{2}+1\right|<0.190 \\
\text { (iv) } \quad 0.183 & <\frac{1}{\sqrt{3}}\left|\omega^{2} A_{l} \lambda_{l}^{3}\left(1-\rho_{l}-i \eta_{l}\right)-\frac{\lambda_{l}^{2}}{2}-\omega \lambda_{l}+1\right|<0.568 \\
\text { (v) } \quad 0.385 & <\frac{1}{\sqrt{3}}\left|-\left(\omega^{2} A_{l}+\frac{\omega}{2}\right) \lambda_{l}^{2}+\lambda_{l}+\omega\right|<0.728 \\
\text { (vi) } 0.613 & <\frac{1}{\sqrt{3}}\left|A_{l} \lambda_{l}^{3}\left(\rho_{l}-i \eta_{l}\right)+\omega A_{l} \lambda_{l}^{2}+\omega^{2}\right|<0.794 \\
\text { (vii) } \quad 0.200 & <\frac{1}{\sqrt{3}}\left|\omega A_{l} \lambda_{l}^{3}\left(1-\rho_{l}-i \eta_{l}\right)-\frac{\lambda_{l}^{2}}{2}-\omega^{2} \lambda_{l}+1\right|<0.576 \\
\text { (viii) } \quad 0.408 & <\frac{1}{\sqrt{3}}\left|-\left(\omega A_{l}+\frac{\omega^{2}}{2}\right) \lambda_{l}^{2}+\lambda_{l}+\omega^{2}\right|<0.742 \\
\text { (ix }) \quad 0.589 & <\frac{1}{\sqrt{3}}\left|A_{l} \lambda_{l}^{3}\left(\rho_{l}-i \eta_{l}\right)+\omega^{2} A_{l} \lambda_{l}^{2}+\omega\right|<0.775
\end{aligned}
$$

Solving these equations up to $O\left(\lambda^{2}\right)$ we get

$$
\begin{aligned}
&-4.8517<A_{l}<-4.4580 \\
&-0.2404<\lambda_{l}<-0.1882 \\
&-5.6339<\rho_{l}<-5.5712 \\
&-4.7160<\eta_{l}<4.8912
\end{aligned}
$$

\subsection{Toward charged lepton masses}

The knowledge of $U_{l L}$ alone does not allow us to determine the charged lepton mass matrix $\mathcal{M}_{l}$ for we need also $U_{l R}$. On the other hand, we can use $U_{l L}$ to diagonalize $\mathcal{M}_{l} \mathcal{M}_{l}^{\dagger}$ as follows. 


$$
U_{l L}^{\dagger} \mathcal{M}_{l} \mathcal{M}_{l}^{\dagger} U_{l L}=\left(\begin{array}{ccc}
m_{e}^{2} & 0 & 0 \\
0 & m_{\mu}^{2} & 0 \\
0 & 0 & m_{\tau}^{2}
\end{array}\right)
$$

giving

$$
\mathcal{M}_{l} \mathcal{M}_{l}^{\dagger}=U_{l L} \cdot\left(\begin{array}{ccc}
m_{e}{ }^{2} & 0 & 0 \\
0 & m_{\mu}^{2} & 0 \\
0 & 0 & m_{\tau}^{2}
\end{array}\right) \cdot U_{l L}^{\dagger}
$$

Up to the order of $\lambda^{2}$ we can approximate $\mathcal{M}_{l} \mathcal{M}_{l}^{\dagger}$ to be of the form

$$
\mathcal{M}_{l} \mathcal{M}_{l}^{\dagger}=\left(\begin{array}{ccc}
\left(1-\lambda_{l}^{2}\right) m_{e}^{2}+\lambda_{l}^{2} m_{\mu}^{2} & \lambda_{l}\left(m_{\mu}{ }^{2}-m_{e}{ }^{2}\right) & 0 \\
\lambda_{l}\left(m_{\mu}{ }^{2}-m_{e}{ }^{2}\right) & \lambda_{l}^{2} m_{e}{ }^{2}+\left(1-\lambda_{l}^{2}\right) m_{\mu}{ }^{2} & A_{l} \lambda_{l}^{2}\left(m_{\tau}{ }^{2}-m_{\mu}{ }^{2}\right) \\
0 & A_{l} \lambda_{l}^{2}\left(m_{\tau}{ }^{2}-m_{\mu}{ }^{2}\right) & m_{\tau}{ }^{2}
\end{array}\right)
$$

From Eq. 5.40, we can see that $\mathcal{M}_{l} \mathcal{M}_{l}^{\dagger}$ is determined completely by the experimental values of $m_{e}, m_{\mu}, m_{\tau}, \lambda_{l}$ and $A_{l}$. Notice that, in the degenerate case $m_{e}=m_{\mu}=m_{\tau}=m, \mathcal{M}_{l} \mathcal{M}_{l}^{\dagger}$ is reduced to a diagonal matrix $\mathcal{M}_{l} \mathcal{M}_{l}^{\dagger}=m^{2} \mathbb{I}$ as one should expect.

A few remarks are in order here. One can view Eq. (5.40) as a constraint equation on the charged lepton mass matrix $\mathcal{M}_{l}$. This constraint equation on $\mathcal{M}_{l} \mathcal{M}_{l}^{\dagger}$ satisfies the experimental constraints on $U_{P M N S}$ as long as $\lambda_{l}$ and $A_{l}$ are within the allowed ranges (5.37). To be able to determine the form of $\mathcal{M}_{l}$, it is clear that one has to impose some kind of symmetry or at the very least make an ansätz on $\mathcal{M}_{l}$ itself as long as $\mathcal{M}_{l} \mathcal{M}_{l}^{\dagger}$ satisfies Eq. 5.40.

Based on the above discussion, it is tempting to propose a similar ansätz for the quark sector for the following reason. The charged leptons as well as the quarks obtain their masses through the couplings with the Higgs doublet $\Phi_{2}$. It might not be unreasonable to speculate that whatever mechanism giving rise to mass mixings in mass matrices could be similar for both quarks and 
charged leptons. One might have

$$
\begin{aligned}
U_{l L} & \rightarrow U_{d L} \\
\lambda_{l}, A_{l}, \rho_{l}, \eta_{l} & \rightarrow \lambda_{d}, A_{d}, \rho_{d}, \eta_{d} \\
U_{l L} & \rightarrow U_{u L} \\
\lambda_{l}, A_{l}, \rho_{l}, \eta_{l} & \rightarrow \lambda_{u}, A_{u}, \rho_{u}, \eta_{u}
\end{aligned}
$$

With the knowledge of $V_{C K M}=U_{u L}^{\dagger} U_{d L}$ 88, one can constraint the above parameters. Furthermore, $\mathcal{M}_{u} \mathcal{M}_{u}^{\dagger}$ and $\mathcal{M}_{d} \mathcal{M}_{d}^{\dagger}$ could have similar forms to the right-hand side of Eq. 5.40 with the replacements $\lambda_{l}, A_{l} \rightarrow \lambda_{u}, A_{u}, m_{e}, m_{\mu}, m_{\tau} \rightarrow m_{u}, m_{c}, m_{t}$ and $\lambda_{l}, A_{l} \rightarrow \lambda_{d}, A_{d}, m_{e}, m_{\mu}, m_{\tau} \rightarrow$ $m_{d}, m_{s}, m_{b}$ respectively. This will be treated in Chapter 7.

\subsection{Summary of the chapter}

We have presented in this manuscript a model of neutrino masses and mixings based on the discrete symmetry group $A_{4}$ as applied to the electroweak(EW)-scale right-handed neutrino model of [70]. In particular, this $A_{4}$ symmetry is applied to the Higgs singlets which are responsible for the neutrino Dirac masses of the EW-scale $\nu_{R}$ model with the aim of obtaining a particular form of matrix namely $U_{C W}$ (Eq. (5.6)), which plays a crucial role in $U_{P M N S}$. The Higgs singlet was introduced in $\left[70\right.$ in order to give the Dirac part of the neutrino masses. By applying the $A_{4}$ symmetry to this sector, we found that the Higgs singlet is increased from one in the original model to four i.e. $\underline{1}+\underline{3}$ of $A_{4}$. The diagonalization of the neutrino Dirac mass matrix generated by the Yukawa coupling of the left-handed doublets $\left(\nu_{L}, e_{L}\right) \sim \underline{3}$, the right-handed doublets $\left(\nu_{R}, e_{R}^{M}\right) \sim \underline{3}$ with these four Higgs singlets is found to be realized by the matrix $U_{\nu}=U_{C W}^{\dagger}$ (Eq. (5.10)). This is in contrast with many popular $A_{4}$-based models where this type of matrix is the one that diagonalizes the charged lepton mass matrix. This is our first step in getting to $U_{P M N S}$ namely 
$U_{P M N S}=U_{\nu}^{\dagger} U_{l L}$. In obtaining $U_{\nu}$, we also derive a couple of sum rules concerning the Dirac masses of the neutrinos. These might turn out to be useful in future studies of neutrino oscillations.

One particular interesting feature of this scheme is the fact that $U_{\nu}$ is generated by the Higgs singlets which do not affect the known properties of the newly discovered 125-GeV SM-like scalar [73]. Notice that scenarios involving more than two Higgs doublets might encounter very very tight constraints which may be hard to satisfy.

The second piece of $U_{P M N S}$, namely $U_{l L}$, comes from the breaking of the $A_{4}$ symmetry in the charged lepton sector as we have shown. It is proportional to the unit matrix in the exact symmetry case (degenerate charged leptons). We take a phenomenological approach by parametrizing the deviation from the unit matrix in terms of a Wolfenstein-like unitary matrix (Eq. (5.31)). We obtain constraints on the parameters of that matrix by using the experimental values of $U_{P M N S}$. Since $U_{l L}$ diagonalizes the lepton mass matrix "squared", namely $U_{l L}^{\dagger} \mathcal{M}_{l} \mathcal{M}_{l}^{\dagger} U_{l L}$, we obtain an equation for $\mathcal{M}_{l} \mathcal{M}_{l}^{\dagger}$ (Eq. (5.40) whose right-hand side is determined entirely by experimental values of the charged lepton masses and the phenomenologically extracted parameters of $U_{l L}$.

As shown in $[70$ and in this manuscript, the sources of masses for the neutrinos and for the charged leptons are entirely different from each other: Higgs singlets and triplet for the neutrinos and Higgs doublet for the charged leptons. Since the quarks also obtain their masses from the Higgs doublet and since $V_{C K M}$ deviates a "little" from the unit matrix, we postulate that $U_{u L}$ and $U_{d L}$ which appear in $V_{C K M}=U_{u L}^{\dagger} U_{d L}$ have the same form as $U_{l L}$ but endowed with their own parameters. In this context, it is very appealing to see why $U_{P M N S}$ is very different from $V_{C K M}$. 


\section{Chapter 6}

\section{Phenomenological Implications of the Lepton Sector}

In this chapter we will discuss two interesting phenomenological consequences of the model of neutrino masses and mixings which are lepton flavor violating (LFV) processes: $\mu \rightarrow e \gamma$ and $\mu-e$ conversion. As is well known, lepton flavor is an accidental conserved quantity in Standard Model (SM) with strictly massless neutrinos. For example, a muon never decays radiatively into an electron plus a photon and neutrinos do not oscillate in SM. However various experiments have now established firmly that neutrinos do oscillate from one flavor to another. The common wisdom, motivated by the physics of $K-\bar{K}$ oscillation in the kaon system, is to give tiny masses with small mass differences to the various light neutrino species. Radiative decay of the muon into electron is then possible but with an unobservable rate highly suppressed with the minuscule neutrino masses 89,90 . Searches for lepton flavor violating rare processes in high intensity experiments are thus important for new physics beyond the SM.

The above LFV processes are presented in details in the following two sections.

The first section contains an updated analysis for the general one-loop induced lepton flavor violating radiative decays $l_{i} \rightarrow l_{j} \gamma$ in an extended mirror model. We then consider a case for the specific $\mu \rightarrow e \gamma$. Mixing effects of the neutrinos and charged leptons constructed with a horizontal $A_{4}$ symmetry are also taken into account. Current experimental limit and projected sensitivity on the branching ratio of $\mu \rightarrow e \gamma$ are used to constrain the parameter space of the model. Calculations 
of two related observables, the electric and magnetic dipole moments of the leptons, are included. We also discussed implications concerning the possible detection of mirror leptons at the LHC and the ILC.

The second sections focuses on the muon-to-electron $(\mu-e)$ conversion in nuclei like aluminum, titanium and gold, which is studied in the context of a class of mirror fermion model with non-sterile right-handed neutrinos having mass at the electroweak scale. At the limit of zero momentum transfer and large mirror lepton masses, we derive a simple formula to relate the conversion rate with the on-shell radiative decay rate of muon into electron. Current experimental limits (SINDRUM II) and projected sensitivities (Mu2e, COMET and PRISM) for the muon-to-electron conversion rates in various nuclei and latest limit from MEG for the radiative decay rate of muon into electron are used to put constraints on the parameter space of the model. Depending on the nuclei targets used in different experiments, for the mirror lepton mass in the range of 100 to $800 \mathrm{GeV}$, the sensitivities of the new Yukawa couplings one can probe in the near future are in the range of one tenth to one hundred-thousandth, depending on the mixing scenarios in the model.

\subsection{One-loop induced LFV radiative decays $\mu \rightarrow e \gamma$}

The EW-scale $\nu_{R}$ model presented in the chapter 4 entails extra $S U(2)$ chiral doublets (the mirror fermions) which have many consequences. These mirror fermions enter loop corrections to various quantities and processes such as the electroweak precision parameters, rare processes, etc.

The first type of effects that needs to be examined is the contributions of these extra chiral doublets to the electroweak precision parameters. These calculations have been performed in 71 and it was found that there is a large parameter space where the EW-scale $\nu_{R}$ model satisfies the EW precision constraints. In a nutshell, the contributions from the mirror fermions are partially cancelled by those of the scalar sector, in particular the $S U(2)$ triplet scalar. 
The next place where mirror fermions enter through loop corrections is rare processes such as $\mu \rightarrow e \gamma$ and $\tau \rightarrow \mu \gamma$. In [91], such processes have been discussed in a generic fashion, with an emphasis on the possible correlation between the observability of the aforementioned rare processes and the decay lengths of the mirror charged leptons, both of which are of phenomenological interests. In this thesis, we will present an update of the process $\mu \rightarrow e \gamma$ taking into account recent developments of the model, including experimental inputs from the recently-discovered $125 \mathrm{GeV}$ SM-like scalar [4,5]. They are summarized below.

Most importantly for this section is the recent work [92] concerning neutrino and SM charged lepton masses and mixings. The fact that the SM lepton mixing matrix $U_{\mathrm{PMNS}}$ (the PontecorvoMaki-Nakagawa-Sakata mixing matrix) is so different from the quark counterpart, $V_{\mathrm{CKM}}$ (the Cabibbo-Kobayashi-Maskawa mixing matrix), has given rise to many models, many of which invoke the presence of some kind of discrete symmetry. Among these different proposals for the discrete symmetry is the popular $A_{4}$ symmetry which has been used to reproduce the tribimaximal form of $U_{\mathrm{PMNS}}$. This symmetry is usually applied to the charged lepton sector [83] and involves four or more Higgs doublets. (Such a large number of Higgs doublets might be hard to accommodate the $125 \mathrm{GeV}$ SM-like scalar with the desired observed properties.) The new twist of 92 is to exhibit the $A_{4}$ symmetry in the neutrino Dirac mass sector and the scalar sector involved is composed of $S U(2) \times U(1)_{Y}$-singlet scalars which are not constrained by LHC data. These singlet scalars are composed of a singlet and a triplet of $A_{4}$. This model reproduces the desired PMNS matrix and makes predictions on the charged lepton mass matrix in the form of $\mathcal{M}_{l} \mathcal{M}_{l}^{\dagger}$. The singlet scalars play a crucial role in the process $\mu \rightarrow e \gamma$ in the EW-scale $\nu_{R}$ model as shown in [91] and updated below in light the aforementioned developments. The results presented in this section contain a deep correlation between the branching ratio $B(\mu \rightarrow e \gamma)$ and the neutrino sector in the form of the PMNS matrix for both normal and inverted hierarchies, as well as the form of the mirror lepton mixing matrix. It will be shown that the exclusion zones in the plots of the branching ratio of $B(\mu \rightarrow e \gamma)$ versus the Yukawa coupling strengths to the singlets depend a bit on how strong the 
$A_{4}$-triplet scalars couple to the leptons.

This section is organized as follows. First, in section 6.1.1, we summarize very briefly the results of neutrino and charged lepton masses and mixings [92. Next, in section 6.1.2 we proceed with the actual calculations of the general process $l_{i} \rightarrow l_{j} \gamma$, the anomalous magnetic dipole moment $\Delta a_{l_{i}}$ and the electric dipole moment $d_{l_{i}}$ for the lepton $l_{i}$. We then perform detailed numerical analysis on the specific $\mu \rightarrow e \gamma$ will be in section 6.1.3. Implications of our results concerning the possible detection of mirror leptons at the LHC and the ILC are discussed in section 6.1.4. We finally summarize and conclude in section 6.1.5. A few useful formulas are collected in Appendix A.

\subsubsection{Review of neutrino and charged lepton masses and mixings in the EW-scale $\nu_{R}$ model}

Since the ideas and notations coming out of this review will be important for the calculation of the rate of $\mu \rightarrow e \gamma$, we will present a little more details here. In 92, a model of the Dirac part of neutrino masses was constructed using the widely popular $A_{4}$ symmetry. Under $A_{4},(\nu, l)_{L}$, $\left(\nu, l^{M}\right)_{R}, e_{R}$ and $e_{L}^{M}$ transform as $\underline{3}$, where $e$ and $\nu$ are generic notations for the charged and neutral leptons. Using the $A_{4}$ multiplication rule $\underline{3} \times \underline{3}=\underline{1}(11+22+33)+\underline{1}^{\prime}\left(11+\omega^{2} 22+\omega 33\right)+\underline{1}^{\prime \prime}(11+$ $\left.\omega 22+\omega^{2} 33\right)+\underline{3}(23,31,12)+\underline{3}(32,13,21)$ with $\omega=e^{i 2 \pi / 3}$, it was argued in 92 that the appropriate set of singlet scalars is composed of an $A_{4}$ singlet $\phi_{0 S}$ and an $A_{4}$-triplet $\left\{\phi_{i S}\right\}(i=1,2,3)$. To reflect the two different ways that the $A_{4}$-triplet can couple to the leptons, 92 wrote down the Lagrangian

$$
\mathcal{L}_{S}=-\bar{l}_{L}^{0}\left(g_{0 S} \phi_{0 S}+g_{1 S} \tilde{\phi}_{S}+g_{2 S} \tilde{\phi}_{S}\right) l_{R}^{M, 0}+\text { H.c. }
$$

where $l_{L}^{0}$ and $l_{R}^{M, 0}$ are gauge eigenstates which are related to the mass eigenstates by

$$
l_{L}^{0}=U_{L}^{l} l_{L}, \quad l_{R}^{M, 0}=U_{R}^{l^{M}} l_{R}^{M}
$$


For the purpose of the subsequent sections, we rewrite Eq. (6.1) as follows

$$
\begin{aligned}
\mathcal{L}_{S} & =-\bar{l}_{L} U_{L}^{l \dagger} U_{\nu} U_{\nu}^{\dagger} M_{\phi} U_{\nu} U_{\nu}^{\dagger} U_{R}^{l^{M}} l_{R}^{M}+\text { H.c. } \\
& =-\bar{l}_{L} U_{\mathrm{PMNS}}^{\dagger} \tilde{M}_{\phi} U_{\mathrm{PMNS}}^{M} l_{R}^{M}+\text { H.c. }
\end{aligned}
$$

where

$$
\tilde{M}_{\phi}=U_{\nu}^{\dagger} M_{\phi} U_{\nu}
$$

and

$$
U_{\mathrm{PMNS}}^{M}=U_{\nu}^{\dagger} U_{R}^{l^{M}}
$$

The above construction can be straightforwardly generalized for the right-handed leptons and lefthanded mirror leptons. Hence the total $\mathcal{L}_{S}$ becomes

$$
\mathcal{L}_{S}=-\bar{l}_{L} U_{\mathrm{PMNS}}^{\dagger} \tilde{M}_{\phi} U_{\mathrm{PMNS}}^{M} l_{R}^{M}-\bar{l}_{R} U_{\mathrm{PMNS}}^{\prime \dagger} \tilde{M}_{\phi}^{\prime} U_{\mathrm{PMNS}}^{\prime M} l_{L}^{M}+\text { H.c. }
$$

where $\tilde{M}_{\phi}^{\prime}=U_{\nu}^{\dagger} M_{\phi}^{\prime} U_{\nu}$ and $M_{\phi}^{\prime}$ is the same as $M_{\phi}$ given by Eq. 5.8 with $g_{0 S} \rightarrow g_{0 S}^{\prime}, g_{1 S} \rightarrow g_{1 S}^{\prime}$ and $g_{2 S} \rightarrow g_{2 S}^{\prime}$. Reality of the eigenvalues of $M_{\phi}^{\prime}$ also implies $g_{2 S}^{\prime}=g_{1 S}^{\prime *}$. In analogous to $U_{\mathrm{PMNS}}$ and $U_{\mathrm{PMNS}}^{M}$, we have defined the following mixing matrices for the second term of Eq. 6.7

$$
U_{\mathrm{PMNS}}^{\prime}=U_{\nu}^{\dagger} U_{R}^{l}
$$

and

$$
U_{\mathrm{PMNS}}^{\prime M}=U_{\nu}^{\dagger} U_{L}^{l^{M}}
$$

where $U_{R}^{l}$ and $U_{L}^{l^{M}}$ are the unitary matrices relating the gauge eigenstates and the mass eigenstates

$$
l_{R}^{0}=U_{R}^{l} l_{R}, \quad l_{L}^{M, 0}=U_{L}^{l^{M}} l_{L}^{M}
$$

\subsubsection{The calculation}

The one-loop irreducible diagram for $l_{i} \rightarrow l_{j} \gamma$ is shown in Fig. (6.1). Other two diagrams not shown are reducible associated with the one-loop dressing for the external fermion lines. They 
are crucial for the cancellation of ultraviolet divergences and gauge invariance in our calculation. The relevant Yukawa couplings between the leptons, mirror leptons and the $A_{4}$ singlet and triplet scalars can be deduced by recasting the Lagrangian $\mathcal{L}_{S}$ in Eq. (6.7) into the following component form

$$
\mathcal{L}_{S}=-\sum_{k=0}^{3} \sum_{i, m=1}^{3}\left(\bar{l}_{L i} \mathcal{U}_{i m}^{L k} l_{R m}^{M}+\bar{l}_{R i} \mathcal{U}_{i m}^{R k} l_{L m}^{M}\right) \phi_{k S}+\text { H.c. }
$$

where

$$
\begin{aligned}
\mathcal{U}_{i m}^{L k} & \equiv\left(U_{\mathrm{PMNS}}^{\dagger} \cdot M^{k} \cdot U_{\mathrm{PMNS}}^{l^{M}}\right)_{i m}, \\
& =\sum_{j, n=1}^{3}\left(U_{\mathrm{PMNS}}^{\dagger}\right)_{i j} M_{j n}^{k}\left(U_{\mathrm{PMNS}}^{M}\right)_{n m},
\end{aligned}
$$

and

$$
\begin{aligned}
\mathcal{U}_{i m}^{R k} & \equiv\left(U_{\mathrm{PMNS}}^{\prime \dagger} \cdot M^{\prime k} \cdot U_{\mathrm{PMNS}}^{\prime l^{M}}\right)_{i m} \\
& =\sum_{j, n=1}^{3}\left(U_{\mathrm{PMNS}}^{\prime \dagger}\right)_{i j} M_{j n}^{\prime k}\left(U_{\mathrm{PMNS}}^{\prime M}\right)_{n m}
\end{aligned}
$$

The matrix elements for the four matrices $M^{k}(k=0,1,2,3)$ are listed in Table I. $M_{j n}^{\prime k}$ can be obtained from $M_{j n}^{k}$ listed in Table I with the following substitutions $g_{0 S} \rightarrow g_{0 S}^{\prime}$ and $g_{1 S} \rightarrow g_{1 S}^{\prime}$.

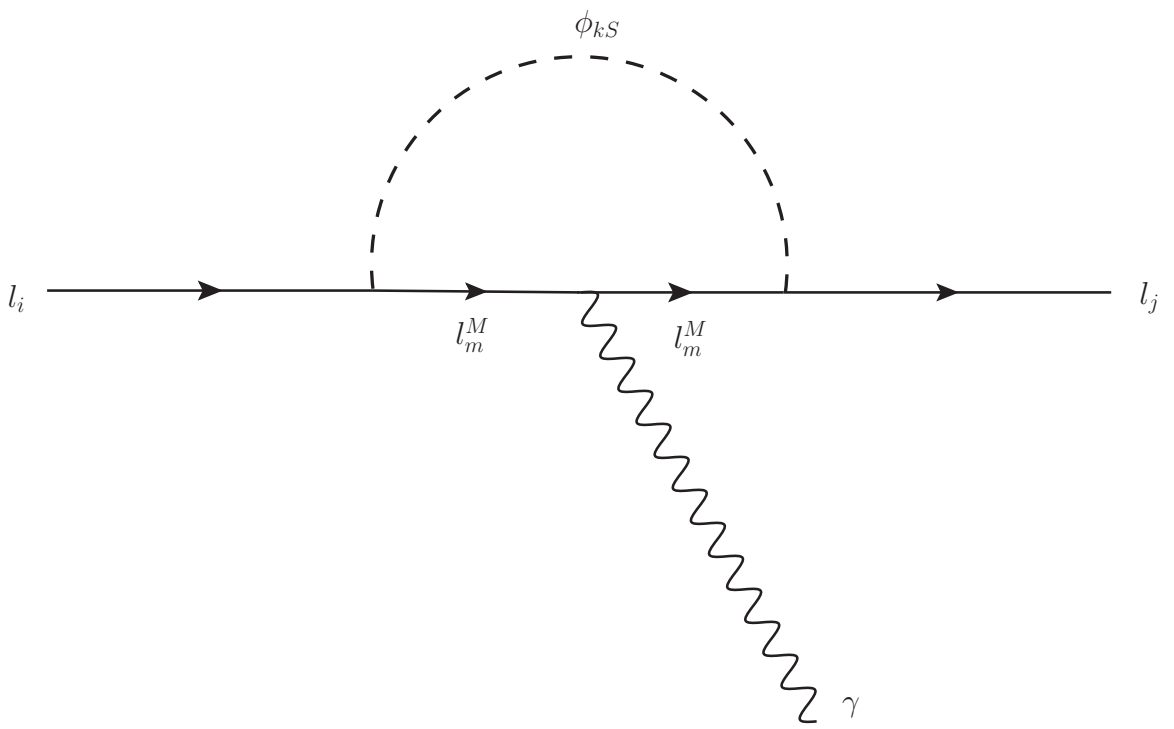

Figure 6.1: One-loop induced Feynman diagram for $l_{i} \rightarrow l_{j} \gamma$ in EW-scale $\nu_{R}$ model. 
Table 6.1: Matrix elements for $M^{k}(k=0,1,2,3)$.

\begin{tabular}{|c|c|}
\hline$M_{j n}^{k}$ & Value \\
\hline \hline$M_{12}^{0}, M_{13}^{0}, M_{21}^{0}, M_{23}^{0}, M_{31}^{0}, M_{32}^{0}$ & 0 \\
$M_{11}^{0}, M_{22}^{0}, M_{33}^{0}$ & $g_{0 S}$ \\
$M_{11}^{1}, M_{11}^{2}, M_{11}^{3}$ & $\frac{2}{3} \operatorname{Re}\left(g_{1 S}\right)$ \\
$M_{22}^{1}, M_{22}^{2}, M_{22}^{3}$ & $\frac{2}{3} \operatorname{Re}\left(\omega^{*} g_{1 S}\right)$ \\
$M_{33}^{1}, M_{33}^{2}, M_{33}^{3}$ & $\frac{2}{3} \operatorname{Re}\left(\omega g_{1 S}\right)$ \\
$M_{12}^{1}, M_{21}^{1}$ & $\frac{2}{3} \operatorname{Re}\left(\omega g_{1 S}\right)$ \\
$M_{12}^{2}, M_{21}^{3}$ & $\frac{1}{3}\left(g_{1 S}+\omega g_{1 S}^{*}\right)$ \\
$M_{12}^{3}, M_{21}^{2}$ & $\frac{1}{3}\left(g_{1 S}^{*}+\omega^{*} g_{1 S}\right)$ \\
$M_{13}^{1}, M_{31}^{1}$ & $\frac{2}{3} \operatorname{Re}\left(\omega^{*} g_{1 S}\right)$ \\
$M_{13}^{2}, M_{31}^{3}$ & $\frac{1}{3}\left(g_{1 S}+\omega^{*} g_{1 S}^{*}\right)$ \\
$M_{13}^{3}, M_{31}^{2}$ & $\frac{1}{3}\left(g_{1 S}^{*}+\omega g_{1 S}\right)$ \\
$M_{23}^{1}, M_{32}^{1}$ & $\frac{2}{3} \operatorname{Re}\left(g_{1 S}\right)$ \\
$M_{23}^{2}, M_{32}^{3}$ & $\frac{2 \omega^{*}}{3} \operatorname{Re}\left(g_{1 S}\right)$ \\
$M_{23}^{3}, M_{32}^{2}$ & $\frac{2 \omega}{3} \operatorname{Re}\left(g_{1 S}\right)$ \\
\hline
\end{tabular}

\subsubsection{The process $l_{i} \rightarrow l_{j} \gamma(i \neq j)$ in EW-scale $\nu_{R}$ Model}

Lorentz and gauge invariance dictate the form of the amplitude for the process $l_{i}^{-}(p) \rightarrow$ $l_{j}^{-}\left(p^{\prime}\right)+\gamma(q)$ to be

$$
\mathcal{M}\left(l_{i}^{-} \rightarrow l_{j}^{-} \gamma\right)=\epsilon_{\mu}^{*}(q) \bar{u}_{j}\left(p^{\prime}\right)\left\{i \sigma^{\mu \nu} q_{\nu}\left[C_{L}^{i j} P_{L}+C_{R}^{i j} P_{R}\right]\right\} u_{i}(p)
$$

where $P_{L, R}=\left(1 \mp \gamma_{5}\right) / 2$. The coefficients $C_{L, R}^{i j}$ can be extracted from the one-loop diagram (Fig. (6.1)),

$$
\begin{aligned}
& C_{L}^{i j}=+\frac{e}{16 \pi^{2}} \sum_{k=0}^{3} \sum_{m=1}^{3}\{ \frac{1}{m_{l_{m}^{M}}^{2}}\left[m_{i} \mathcal{U}_{j m}^{R k}\left(\mathcal{U}_{i m}^{R k}\right)^{*}+m_{j} \mathcal{U}_{j m}^{L k}\left(\mathcal{U}_{i m}^{L k}\right)^{*}\right] \mathcal{I}\left(\frac{m_{\phi_{k S}}^{2}}{m_{l_{m}^{M}}^{2}}\right) \\
&\left.+\frac{1}{m_{l_{m}^{M}}} \mathcal{U}_{j m}^{R k}\left(\mathcal{U}_{i m}^{L k}\right)^{*} \mathcal{J}\left(\frac{m_{\phi_{k S}}^{2}}{m_{l_{m}^{M}}^{2}}\right)\right\}, \\
& C_{R}^{i j}=+\frac{e}{16 \pi^{2}} \sum_{k=0}^{3} \sum_{m=1}^{3}\left\{\frac{1}{m_{l_{m}^{M}}^{2}}\left[m_{i} \mathcal{U}_{j m}^{L k}\left(\mathcal{U}_{i m}^{L k}\right)^{*}+m_{j} \mathcal{U}_{j m}^{R k}\left(\mathcal{U}_{i m}^{R k}\right)^{*}\right] \mathcal{I}\left(\frac{m_{\phi_{k S}}^{2}}{m_{l_{m}^{M}}^{2}}\right)\right. \\
&\left.+\frac{1}{m_{l_{m}^{M}}} \mathcal{U}_{j m}^{L k}\left(\mathcal{U}_{i m}^{R k}\right)^{*} \mathcal{J}\left(\frac{m_{\phi_{k S}}^{2}}{m_{l_{m}^{M}}^{2}}\right)\right\} .
\end{aligned}
$$


Here we have assumed the mirror lepton masses are much larger than the external fermion masses $m_{l_{m}^{M}} \gg m_{i, j}$ and set $m_{i, j} \rightarrow 0$ in the loop functions $\mathcal{I}(r)$ and $\mathcal{J}(r)$, which are simply given by

$$
\begin{aligned}
\mathcal{I}(r) & =\frac{1}{12(1-r)^{4}}\left[-6 r^{2} \log r+r\left(2 r^{2}+3 r-6\right)+1\right], \\
\mathcal{J}(r) & =\frac{1}{2(1-r)^{3}}\left[-2 r^{2} \log r+r(3 r-4)+1\right] .
\end{aligned}
$$

In our numerical work for $\mu \rightarrow e \gamma$ presented in section 6.1.3, we will consider the mirror lepton masses of the order a few hundred $\mathrm{GeV}$ and the $A_{4}$ singlet and triplet scalar masses of the order $10 \mathrm{MeV}$, thus the ratio $r=m_{\phi_{k S}}^{2} / m_{l_{m}^{M}}^{2} \sim 10^{-8}$ is very tiny. For all practical purposes, one can replace Eqs. (B.1) and (B.2) by the limits $\lim _{r \rightarrow 0} \mathcal{I}(r)=1 / 12$ and $\lim _{r \rightarrow 0} \mathcal{J}(r)=1 / 2$ respectively. Formulas of $\mathcal{I}$ and $\mathcal{J}$ for the general case of $m_{i, j} \neq 0$ are given in the Appendix A,

The partial width for $l_{i} \rightarrow l_{j} \gamma$ is given by

$$
\Gamma\left(l_{i} \rightarrow l_{j} \gamma\right)=\frac{1}{16 \pi} m_{l_{i}}^{3}\left(1-\frac{m_{l_{j}}^{2}}{m_{l_{i}}^{2}}\right)^{3}\left(\left|C_{L}^{i j}\right|^{2}+\left|C_{R}^{i j}\right|^{2}\right)
$$

\subsubsection{Magnetic Dipole Moment}

The magnetic dipole moment anomaly for lepton $l_{i}$ can be easily extracted from the above calculation with the following result

$$
\begin{aligned}
\Delta a_{l_{i}}= & \frac{2 m_{l_{i}}}{e}\left(\frac{C_{L}^{i i}+C_{R}^{i i}}{2}\right) \\
= & +\frac{1}{16 \pi^{2}}\left\{\sum_{k=0}^{3} \sum_{m=1}^{3} 2\left(\left|\mathcal{U}_{i m}^{L k}\right|^{2}+\left|\mathcal{U}_{i m}^{R k}\right|^{2}\right) \frac{m_{l_{i}}^{2}}{m_{l_{m}^{M}}^{2}} \mathcal{I}\left(\frac{m_{\phi_{k S}}^{2}}{m_{l_{m}^{M}}^{2}}\right)\right. \\
& \left.\quad+\sum_{k=0}^{3} \sum_{m=1}^{3} \operatorname{Re}\left(\mathcal{U}_{i m}^{L k}\left(\mathcal{U}_{i m}^{R k}\right)^{*}\right) \frac{m_{l_{i}}}{m_{l_{m}^{M}}} \mathcal{J}\left(\frac{m_{\phi_{k S}}^{2}}{m_{l_{m}^{M}}^{2}}\right)\right\} .
\end{aligned}
$$




\subsubsection{Electric Dipole Moment}

The electric dipole moment operator for a fermion $f$ is usually defined as

$$
\mathcal{L}_{\mathrm{EDM}}=-i \frac{d_{f}}{2} \bar{f} \sigma^{\mu \nu} \gamma_{5} f F_{\mu \nu}
$$

where $F_{\mu \nu}$ is the electromagnetic field strength and the coefficient $d_{f}$ the electric dipole moment. The electric dipole moment for lepton $l_{i}$ can also be easily extracted from the above calculation with the result

$$
\begin{aligned}
d_{l_{i}} & =\frac{i}{2}\left(C_{L}^{i i}-C_{R}^{i i}\right) \\
& =+\frac{e}{16 \pi^{2}} \sum_{k=0}^{3} \sum_{m=1}^{3} \frac{1}{m_{l_{m}^{M}}} \operatorname{Im}\left(\mathcal{U}_{i m}^{L k}\left(\mathcal{U}_{i m}^{R k}\right)^{*}\right) \mathcal{J}\left(\frac{m_{\phi_{k S}}^{2}}{m_{l_{m}^{M}}^{2}}\right)
\end{aligned}
$$

\subsubsection{Numerical Analysis}

In the previous sections we are showing theoretical analyses for the general case of $l_{i} \rightarrow l_{j} \gamma$ decays. Hereinafter, let us take $i, j$ to be $\mu, e$ in order to tailgate current experimental data for the process $\mu \rightarrow e \gamma$. The branching ratio $\mathrm{B}(\mu \rightarrow e \gamma)$ is then given by

$$
\mathrm{B}(\mu \rightarrow e \gamma)=\tau_{\mu} \cdot \Gamma(\mu \rightarrow e \gamma)
$$

where $\tau_{\mu}$ is the lifetime of the muon 94

$$
\tau_{\mu}=(2.1969811 \pm 0.0000022) \times 10^{-6} \mathrm{~s}
$$

In our numerical analysis, we will adopt the following approach:

- For the masses of the singlet scalars $\phi_{k S}$, we take

$$
m_{\phi_{0 S}}: m_{\phi_{1 S}}: m_{\phi_{2 S}}: m_{\phi_{3 S}}=M_{S}: 2 M_{S}: 3 M_{S}: 4 M_{S}
$$

with a fixed common mass $M_{S}=10 \mathrm{MeV}$. As long as $m_{\phi_{k S}} \ll m_{l_{m}^{M}}$, our results will not be affected much by the exact mass relations among these singlet scalars. 
- For the masses of the mirror lepton $l_{m}^{M}$, we take

$$
m_{l_{m}^{M}}=M_{\text {mirror }}+\delta_{m}
$$

with $\delta_{1}=0, \delta_{2}=10 \mathrm{GeV}, \delta_{3}=20 \mathrm{GeV}$ and vary the common mass $M_{\text {mirror }}$ from $100 \mathrm{GeV}$ to $800 \mathrm{GeV}$.

- We assume all the Yukawa couplings $g_{0 S}, g_{1 S}, g_{2 S}, g_{0 S}^{\prime}, g_{1 S}^{\prime}$, and $g_{2 S}^{\prime}$ to be all rea $!^{1}$. As mentioned before, $g_{2 S}=\left(g_{1 S}\right)^{*}$ and $g_{2 S}^{\prime}=\left(g_{1 S}^{\prime}\right)^{*}$ due to the reality of the mass eigenvalues of the Dirac neutrino masses. For simplicity, we also take $g_{0 S}=g_{0 S}^{\prime}, g_{1 S}=g_{1 S}^{\prime}$ and study the following 6 cases:

(1) $g_{0 S} \neq 0, g_{1 S}=0$. The $A_{4}$ triplet terms are switched off.

(2) $g_{1 S}=10^{-2} \times g_{0 S}$. The $A_{4}$ triplet couplings are merely one percent of the singlet ones.

(3) $g_{1 S}=10^{-1} \times g_{0 S}$. The $A_{4}$ triplet couplings are 10 percent of the singlet ones.

(4) $g_{1 S}=0.5 \times g_{0 S}$. The $A_{4}$ triplet couplings are one half of the singlet ones.

(5) $g_{1 S}=g_{0 S}$. Both $A_{4}$ singlet and triplet terms have the same weight.

(6) $g_{0 S}=0, g_{1 S} \neq 0$. The $A_{4}$ singlet terms are switched off.

- For the three unknown mixing matrices $U_{\mathrm{PMNS}}^{M}, U_{\mathrm{PMNS}}^{\prime}$ and $U_{\mathrm{PMNS}}^{\prime M}$, we will consider two scenarios:

* Scenario 1

$$
U_{\mathrm{PMNS}}^{M}=U_{\mathrm{PMNS}}^{\prime}=U_{\mathrm{PMNS}}^{\prime M}=U_{C W}^{\dagger}
$$

* Scenario 2

$$
U_{\mathrm{PMNS}}^{M}=U_{\mathrm{PMNS}}^{\prime}=U_{\mathrm{PMNS}}^{\prime M}=U_{\mathrm{PMNS}}
$$

\footnotetext{
${ }^{1}$ In this study, we do not analyze the possibility of electric dipole moments for the charged leptons in which complex Yukawa couplings must be assumed.
} 
Recall that the standard parameterization of the PMNS matrix is given by

$$
U_{\mathrm{PMNS}}=\left(\begin{array}{ccc}
c_{12} c_{13} & s_{12} c_{13} & s_{13} e^{-i \delta} \\
-s_{12} c_{23}-c_{12} s_{23} s_{13} e^{i \delta} & c_{12} c_{23}-s_{12} s_{23} s_{13} e^{i \delta} & s_{23} c_{13} \\
s_{12} s_{23}-c_{12} c_{23} s_{13} e^{i \delta} & -c_{12} s_{23}-s_{12} c_{23} s_{13} e^{i \delta} & c_{23} c_{13}
\end{array}\right) \cdot V
$$

where $s_{i j} \equiv \sin \theta_{i j}, c_{i j} \equiv \cos \theta_{i j}$ and $V=\operatorname{Diag}\left(1, e^{i \alpha_{21} / 2}, e^{i \alpha_{31} / 2}\right)$ is the Majorana phase matrix. We will ignore the Majorana phases in this analysis.

In Table 6.2 we list the $1 \sigma$ range of the mixing parameters as given by the recent analysis of global three-neutrino oscillation data in [54,55]. With the central values for the mixing parameters given in Table II as inputs, we obtain two possible solutions of the PMNS matrix:

$$
U_{\mathrm{PMNS}}^{\mathrm{NH}}=\left(\begin{array}{ccc}
0.8221 & 0.5484 & -0.0518+0.1439 i \\
-0.3879+0.07915 i & 0.6432+0.0528 i & 0.6533 \\
0.3992+0.08984 i & -0.5283+0.05993 i & 0.7415
\end{array}\right)
$$

for normal hierarchy, and

$$
U_{\mathrm{PMNS}}^{\mathrm{IH}}=\left(\begin{array}{ccc}
0.8218 & 0.5483 & -0.08708+0.1281 i \\
-0.3608+0.0719 i & 0.6467+0.04796 i & 0.6664 \\
0.4278+0.07869 i & -0.5254+0.0525 i & 0.7293
\end{array}\right)
$$

for inverted hierarchy. For each scenario, we consider these two possible solutions for the $U_{\mathrm{PMNS}}$. Due to the small differences between these two solutions, we expect our results are not too sensitive to the neutrino mass hierarchies.

- Limits on $B(\mu \rightarrow e \gamma)$ from MEG experiment [95] and its projected sensitivity [96]:

$$
\begin{aligned}
& B(\mu \rightarrow e \gamma) \leq 5.7 \times 10^{-13}(90 \text { C.L. })[\mathrm{MEG}, 2013] \\
& B(\mu \rightarrow e \gamma) \sim 4 \times 10^{-14} \text { [Projected Sensitivity] }
\end{aligned}
$$


- $\Delta a_{\mu}$ from E821 experiment 97]:

$$
\Delta a_{\mu} \equiv a_{\mu}^{\exp }-a_{\mu}^{\mathrm{SM}}=288(63)(49) \times 10^{-11}
$$

Table 6.2: Mixing parameters from global three-neutrino oscillation data taken from 54,55].

\begin{tabular}{|c||c|c|}
\hline Mixing Parameters & Normal Hierarchy & Inverted Hierarchy \\
\hline \hline $\sin ^{2} \theta_{12}$ & $0.308 \pm 0.017$ & $0.308 \pm 0.017$ \\
$\sin ^{2} \theta_{23}$ & $0.437_{-0.023}^{+0.033}$ & $0.455_{-0.031}^{+0.139}$ \\
$\sin ^{2} \theta_{13}$ & $0.0234_{-0.0019}^{+0.0020}$ & $0.024_{-0.0022}^{+0.0019}$ \\
$\delta / \pi$ & $1.39_{-0.27}^{+0.38}$ & $1.31_{-0.33}^{+0.29}$ \\
$\delta m^{2}=m_{2}^{2}-m_{1}^{2}$ & $\left(7.54_{-0.22}^{+0.26}\right) \times 10^{-5} \mathrm{eV}^{2}$ & $\left(7.54_{-0.22}^{+0.26}\right) \times 10^{-5} \mathrm{eV}^{2}$ \\
$\Delta m^{2}=\left|m_{3}^{2}-\left(m_{1}^{2}+m_{2}^{2}\right) / 2\right|$ & $(2.43 \pm 0.06) \times 10^{-3} \mathrm{eV}^{2}$ & $(2.38 \pm 0.06) \times 10^{-3} \mathrm{eV}^{2}$ \\
\hline
\end{tabular}

Since the dominant contributions to the loop amplitude arise from the mass insertion of the internal mirror lepton line in Fig. (6.1), only the last terms in Eqs. (6.17), (6.18) and (6.22) are significant numerically. As long as $m_{\phi_{k S}} \ll m_{l_{m}^{M}}$, the current MEG limit (Eq. (6.27)) on the branching ratio $B(\mu \rightarrow e \gamma)$ imposes the constraint

$$
\left|\sum_{k, m} \mathcal{U}_{1 m}^{R k}\left(\mathcal{U}_{2 m}^{L k}\right)^{*}\left(\frac{100 \mathrm{GeV}}{m_{l_{m}^{M}}}\right)\right|^{2}+\left|\sum_{k, m} \mathcal{U}_{1 m}^{L k}\left(\mathcal{U}_{2 m}^{R k}\right)^{*}\left(\frac{100 \mathrm{GeV}}{m_{l_{m}^{M}}}\right)\right|^{2} \leq 7.9 \times 10^{-19},
$$

while the result from the Brookhaven E821 experiment on $\Delta a_{\mu}$ (Eq. (6.29) imposes

$$
\sum_{k, m} \operatorname{Re}\left(\mathcal{U}_{2 m}^{L k}\left(\mathcal{U}_{2 m}^{R k}\right)^{*}\right)\left(\frac{100 \mathrm{GeV}}{m_{l_{m}^{M}}}\right) \leq 8.6 \times 10^{-4} .
$$

In Figs. 6.2 - 6.7) we plot the contour of $\log _{10} B(\mu \rightarrow e \gamma)$ (upper panel) and $\log _{10} \Delta a_{\mu}$ (bottom panel) in the $\left(g_{0 S}\right.$ or $\left.1 S, M_{\text {mirror }}\right)$ plane for both normal (left panel) and inverted (right panel) neutrino mass hierarchies for scenarios 1 (red curves) and 2 (blue curves) with the six cases of couplings aforementioned: (1) $g_{0 S} \neq 0, g_{1 S}=0$ (Fig. 6.2), (2) $g_{1 S}=10^{-2} \times g_{0 S}$ (Fig. 6.3), (3) $g_{1 S}=10^{-1} \times g_{0 S}$ (Fig. 6.4), (4) $g_{1 S}=0.5 \times g_{0 S}$ (Fig. 6.5), (5) $g_{0 S}=g_{1 S}$ (Fig. 6.6), and (6) $g_{0 S}=0, g_{1 S} \neq 0$ (Fig. 6.7), respectively.

At the upper panel of each of these figures, the (light) gray area is excluded by the current limit of $\log _{10} B(\mu \rightarrow e \gamma)=-12.24$ from MEG experiment 95 for scenario (1) 2 respectively. The 

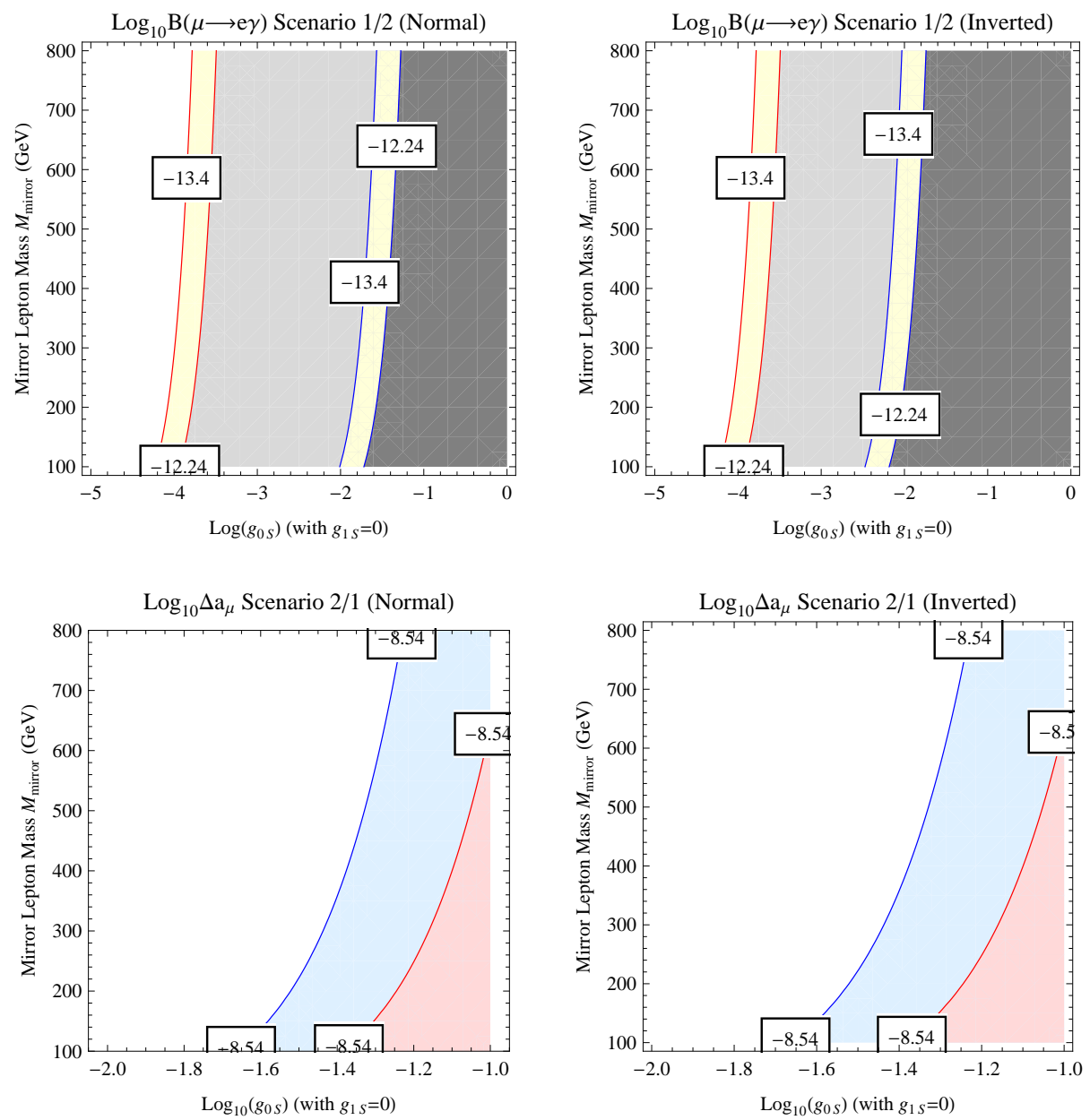

Figure 6.2: Contour plots of $\log _{10} B\left(\mu \rightarrow e \gamma\right.$ ) (top panel) and $\log _{10} \Delta a_{\mu}$ (bottom panel) on the $\left(g_{0 S}, M_{\text {mirror }}\right)$ plane for normal (left panel) and inverted (right panel) hierarchy in scenarios 1 (red curves) and 2 (blue curves) with $g_{0 S}=g_{0 S}^{\prime}$ and $g_{1 S}=g_{1 S}^{\prime}=0$. For details of other input parameters, one can refer to the text in section 6.1.3.

projected sensitivity of $\log _{10} B(\mu \rightarrow e \gamma)=-13.40[96$ is also shown for each scenario in the two plots in the upper panel for comparison.

At the bottom panel of each of these figures, the red (blue) area is defined by the $\log _{10} \Delta a_{\mu}=$ -8.54 97 from the E821 experiment of the Brookhaven National Lab (BNL) for the discrepancy between the SM model prediction and the measurement for the muon anomalous magnetic dipole moment for scenario $1(2)$, respectively. 

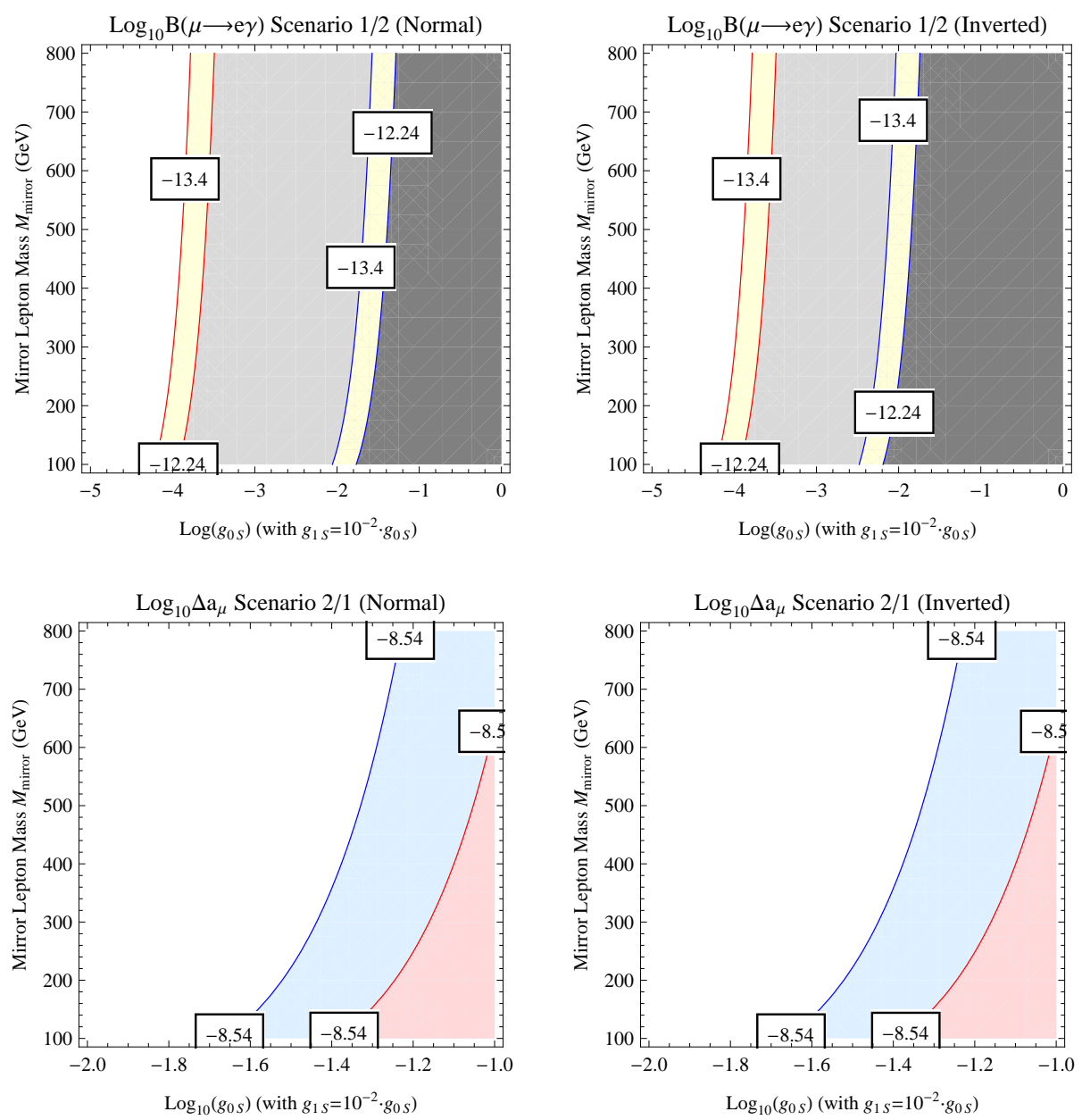

Figure 6.3: Same as Fig. 6.2 with $g_{0 S}=g_{0 S}^{\prime}$ and $g_{1 S}=g_{1 S}^{\prime}=10^{-2} \cdot g_{0 S}$ instead.

From all the plots in these figures, we observe the following general features.

- In the same mass range of the mirror leptons the LFV process $\mu \rightarrow e \gamma$ is more sensitive to the couplings by almost two order of magnitudes as compared with the anomalous magnetic dipole moment of the muon. This is partly due to the fact that the $B(\mu \rightarrow e \gamma)$ is quartic in the couplings, while in $\Delta a_{\mu}$ they are quadratic.

- As one turns on the $A_{4}$ triplet coupling $g_{1 S}$ from 0 to $g_{1 S}=g_{0 S}$ (Fig. (6.2) to Fig. (6.6)), the contours for $\log _{10} B(\mu \rightarrow e \gamma)$ (upper panels) are shifting toward to the left, indicating the role of the triplet singlets become more relevant and thus the constraints on parameter 

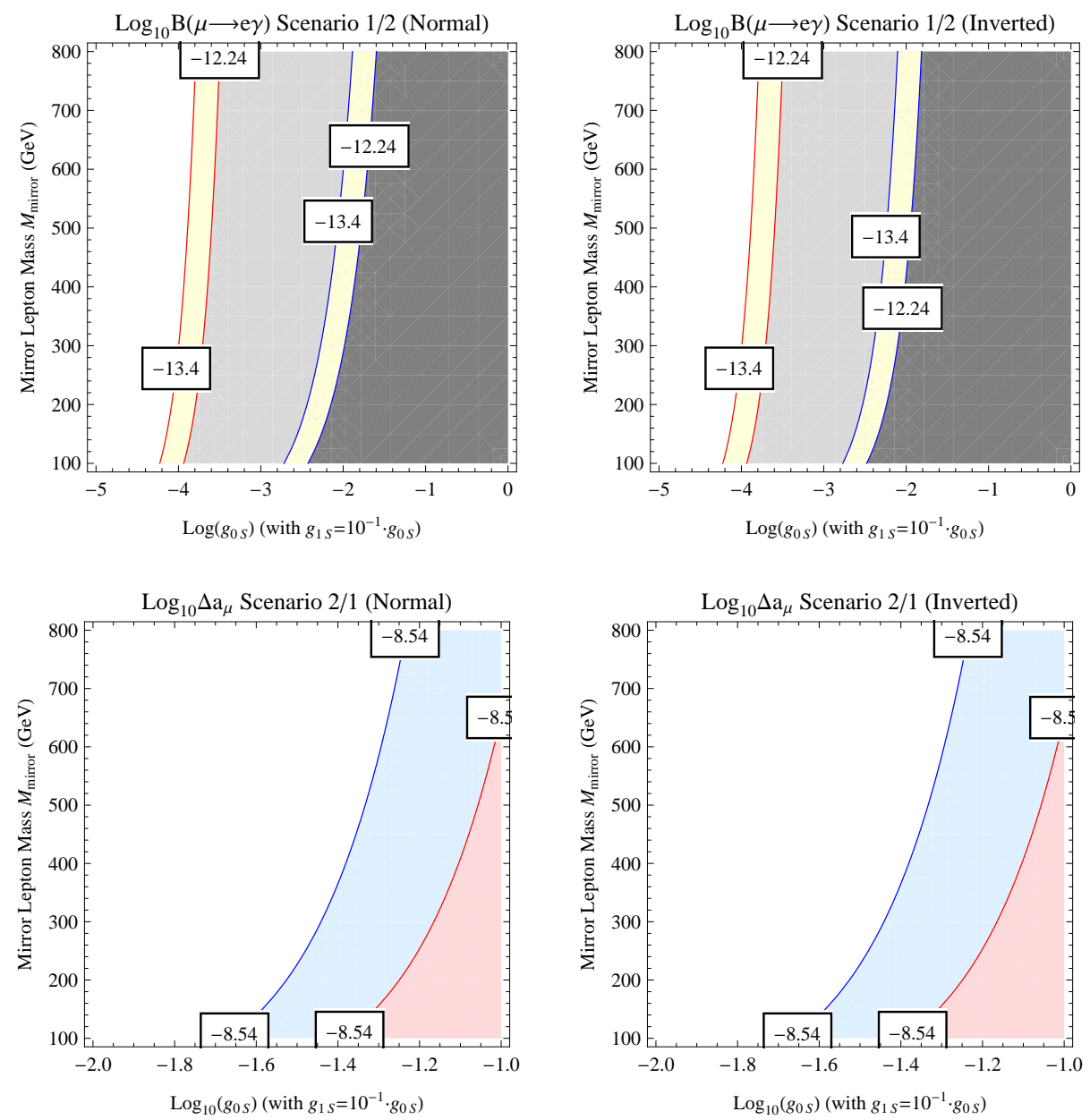

Figure 6.4: Same as Fig. 6.2 with $g_{0 S}=g_{0 S}^{\prime}$ and $g_{1 S}=g_{1 S}^{\prime}=10^{-1} \cdot g_{0 S}$ instead.

space become more stringent from the current MEG limit. However in the last case of Fig. (6.7) when the $A_{4}$ singlet coupling $g_{0 S}$ is set to zero such that only the triplet singlets are contributing in the loop diagram, the contours of $\log _{10} B(\mu \rightarrow e \gamma)$ are slightly shifting back toward to the right. Similar behaviors can be found for the contours of $\log _{10} \Delta a_{\mu}$, but the effects are tiny and not easily seen on the log scale, except for the last three cases of Figs. 6.5)-6.7 (lower panels).

Regarding the sensitivity on the two scenarios, we can obtain the following statement by comparing the red and blue contours corresponding to the scenarios 1 and 2 in each of these 

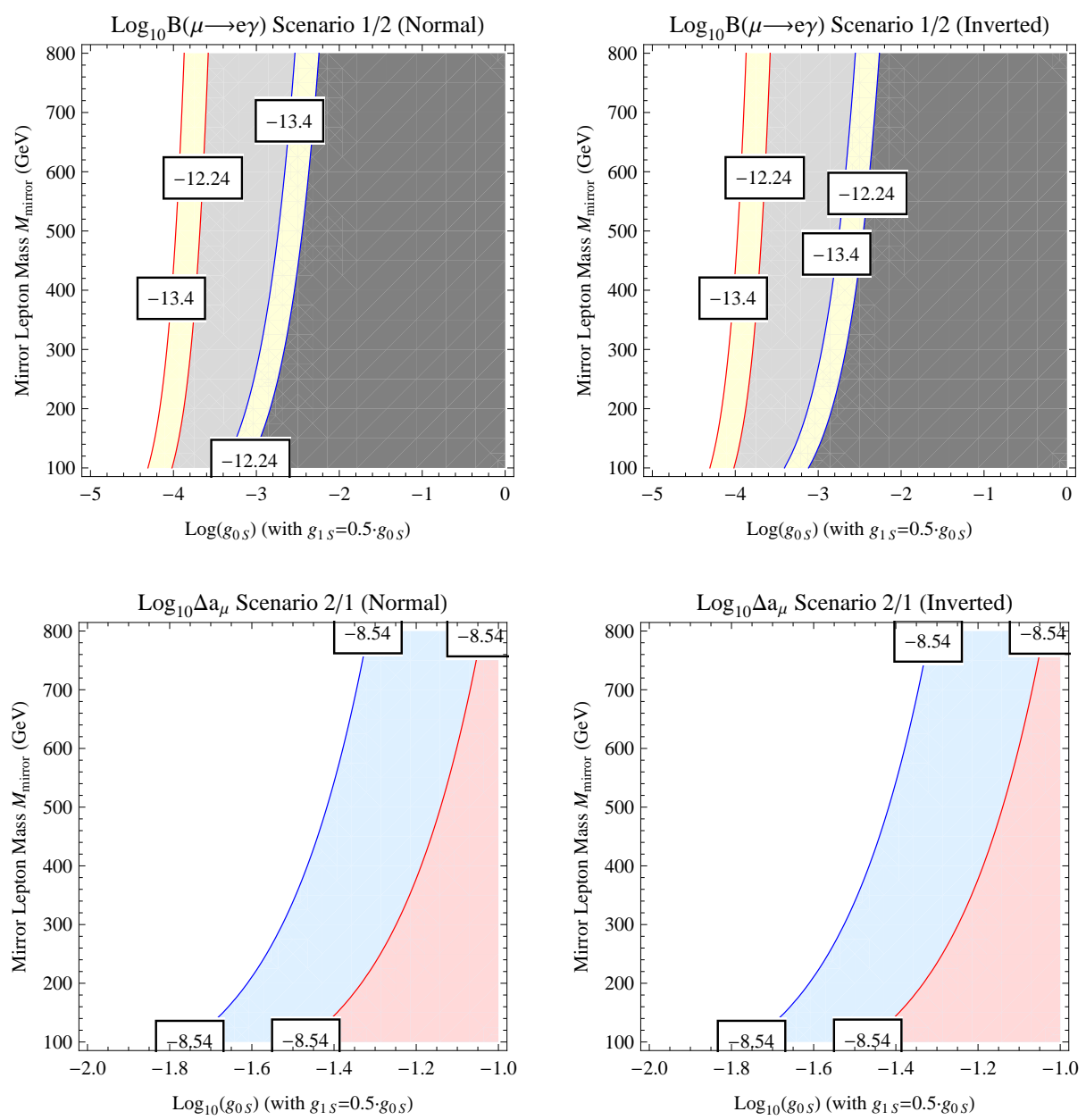

Figure 6.5: Same as Fig. (6.2) with $g_{0 S}=g_{0 S}^{\prime}$ and $g_{1 S}=g_{1 S}^{\prime}=0.5 \cdot g_{0 S}$ instead.

figures.

- The sensitivity of the couplings in the $B(\mu \rightarrow e \gamma)$ has been weakened by one to two order of magnitudes for scenario 2 as compared to scenario 1 . This is due to the fact that in scenario 2 , the three unknown unitary mixing matrices are now departure from $U_{C W}^{\dagger}$, which allows the couplings take on larger values since the amplitudes involve products of the couplings and the elements of mixing matrices. However this sensitivity is not present for the muon anomalous magnetic dipole moment as the distance between the two red and blue contours for the two scenarios in the lower panels of all these plots are well within a small range of 

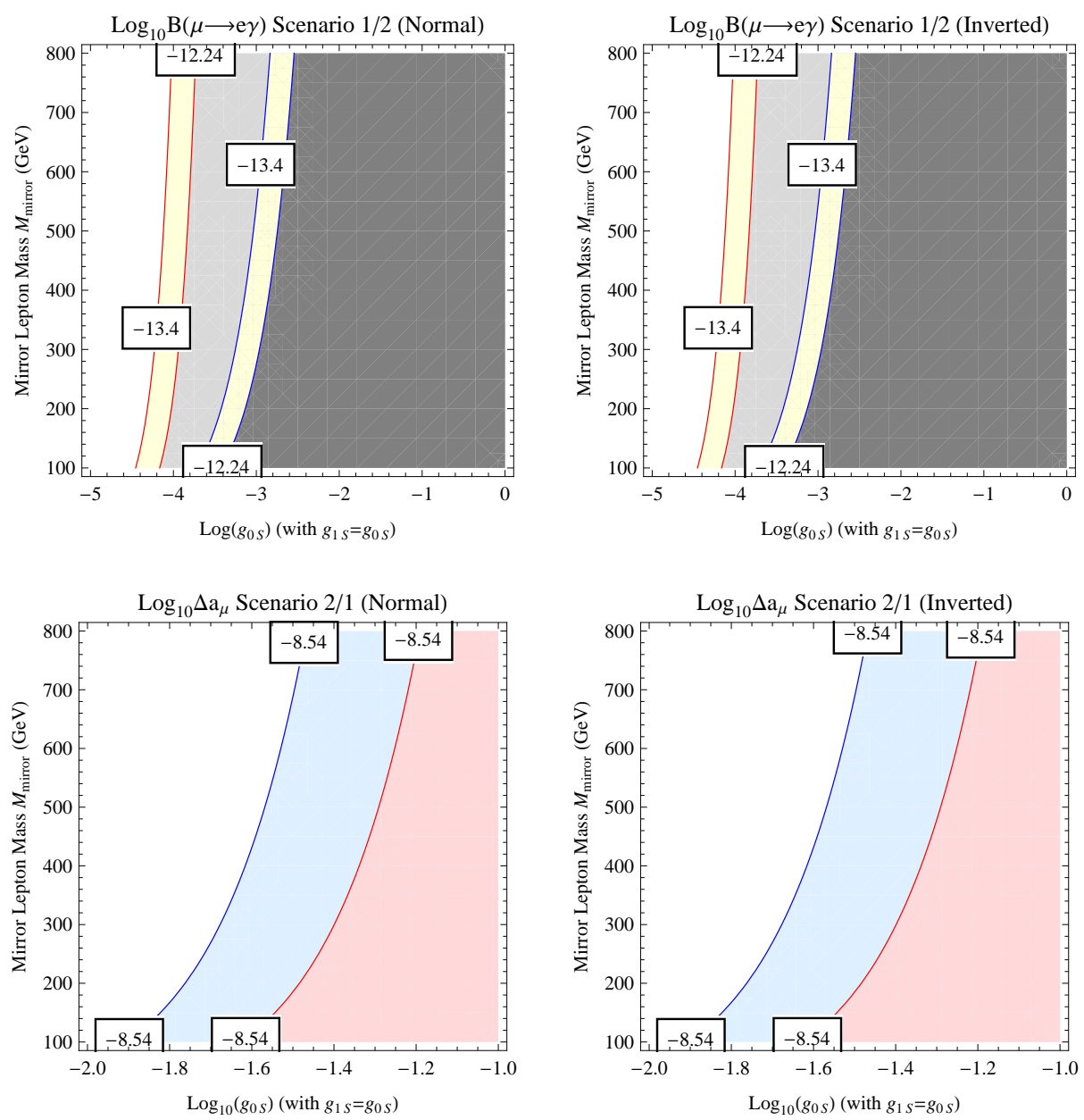

Figure 6.6: Same as Fig. (6.2) with $g_{0 S}=g_{0 S}^{\prime}=g_{1 S}=g_{1 S}^{\prime}$ instead.

the coupling $g_{0 S}$ (or $g_{1 S}$ in Fig. (6.7)). For example, at $M_{\text {mirror }}=100 \mathrm{GeV}$, the allowed value of $g_{0 S}$ varies from $10^{-4.5}$ to $10^{-1.8}\left(10^{-1.9}\right.$ to $\left.10^{-1.4}\right)$ as seen from the upper (lower) panels of Figs. 6.2 - 6.6.

Regarding the sensitivity on the neutrino mass hierarchies, one can obtain the following statements by comparing the left and right panels in each of these figures.

- As one slowly turns on the $A_{4}$ triplet coupling $g_{1 S}=0$ (Fig. (6.2) ) to $g_{1 S}=10^{-1} \times g_{0 S}$ (Fig. (6.4) $)$, the red contours of $\log _{10} B(\mu \rightarrow e \gamma)$ of scenario 1 in the left and right panels 

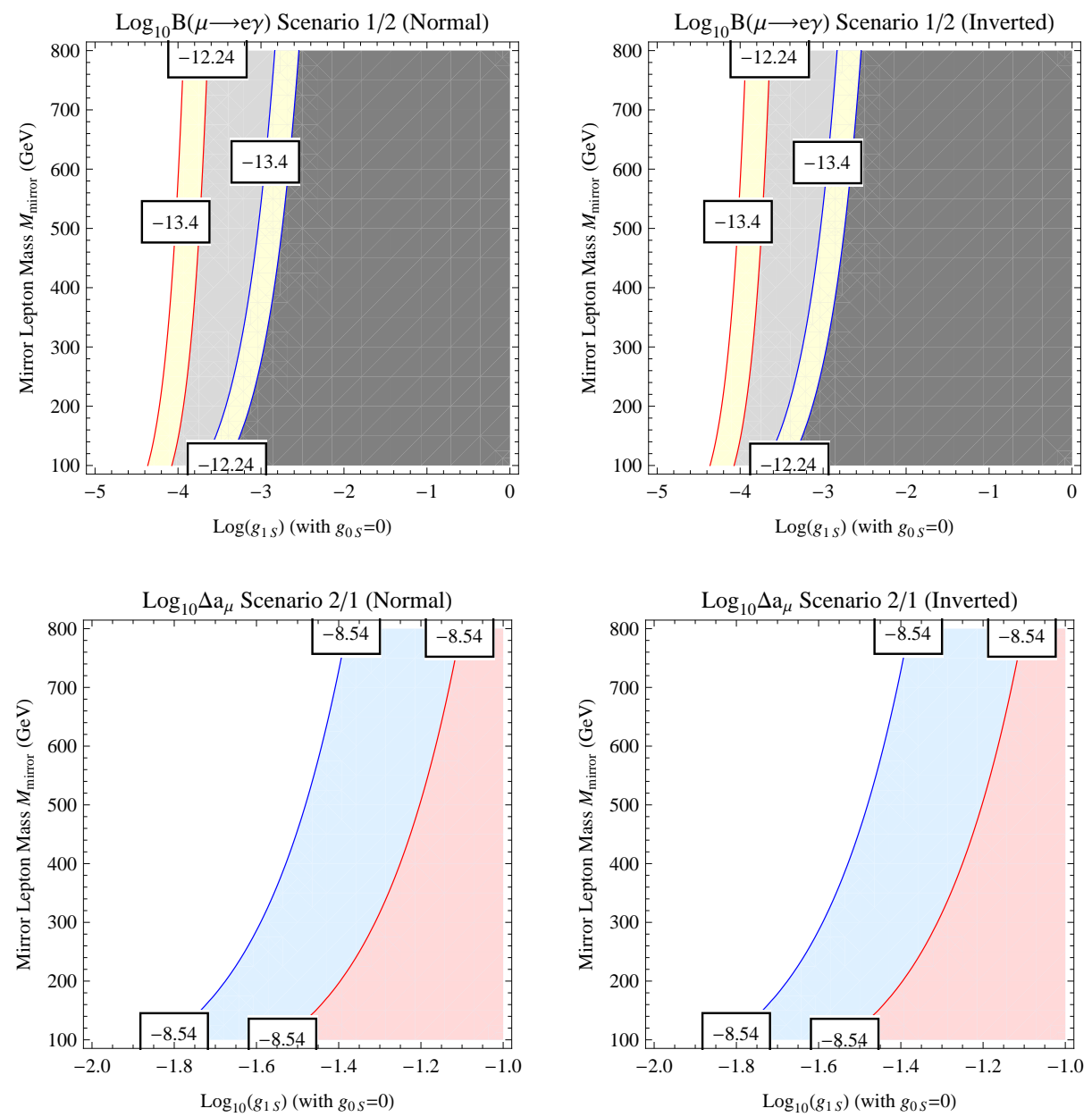

Figure 6.7: Same as Fig. 6.2 with $g_{0 S}=g_{0 S}^{\prime}=0$ and $g_{1 S}=g_{1 S}^{\prime}$ instead.

in all these plots remain the same, while the blue contours of scenario 2 in the right panels move toward to the left. This indicates that noticeable differences in the contours of $\log _{10} B(\mu \rightarrow e \gamma)$ between the normal and inverted neutrino mass hierarchies can be seen in these cases. In general the couplings are about an order of magnitude more sensitive in the inverted mass hierarchy than the normal one for scenario 2 . However, for $g_{1 S} \geq 0.5 \times g_{0 S}$, these differences diminish.

- There are no discernible differences between the two mass hierarchies for the muon anomalous magnetic dipole moment in both scenarios for all 6 cases of couplings. 


\subsubsection{Implications}

The constraints on the Yukawa couplings coming from $\mu \rightarrow e \gamma$ has several implications among which two are particularly relevant.

- The size allowed for the Yukawa couplings by present limits on $B(\mu \rightarrow e \gamma)$ has an important implication on the decay lengths of the mirror leptons. It is beyond the scope of this thesis to discuss this in detail here but a few remarks are in order. In the search for mirror leptons, one would like to look for characteristic signatures which can be distinguished from SM background. One of such signatures could be events with displaced vertices, in particular events with decay lengths which are macroscopic $(l>1 \mathrm{~mm})$. How this type of events can be correlated to $\mu \rightarrow e \gamma$ is a topic which was already mentioned in [91]. With the present update which includes a more detailed analysis taking into account mixings in the lepton sector, one can have a better idea of the correlation between the feasibility to observe $\mu \rightarrow e \gamma$ and the detection of mirror leptons.

A mirror lepton can decay directly into SM leptons with an accompanying Higgs singlet. For example, one can have $l_{R i}^{M} \rightarrow l_{L j}+\phi_{k S}$ where $i, j=e, \mu, \tau$ and $k=0,1,2,3$. The decay length will depend on the magnitude of the Yukawa couplings as well as on the various mixing parameters contained in Eq. 6.7). We just take one example here for the

sake of discussion. The interaction Lagrangian for $\mu_{R i}^{M} \rightarrow l_{L j}+\phi_{k S}$ can be expressed as 
$\left(\bar{e}_{L} \mathcal{M}_{12}+\bar{\mu}_{L} \mathcal{M}_{22}+\bar{\tau}_{L} \mathcal{M}_{32}\right) \mu_{R}^{M}$ where (for scenario 2 with the normal hierarchy)

$$
\begin{aligned}
\mathcal{M}_{12}= & \left(5.834 \times 10^{-6}-0.000025 i\right) g_{0 S} \phi_{0 S}+ \\
& \left(g_{1 S}(0.324+0.159 i)+g_{2 S}(0.407-0.171 i)\right) \phi_{1 S}+ \\
& \left(g_{1 S}(0.154+0.200 i)+g_{2 S}(0.192+0.238 i)\right) \phi_{2 S}+ \\
& \left(g_{1 S}(0.074-0.325 i)+g_{2 S}(0.201-0.102 i)\right) \phi_{3 S} \\
\mathcal{M}_{22}= & 0.999933 g_{0 S} \phi_{0 S}+ \\
& \left(g_{1 S}(-0.262+0.332 i)+g_{2 S}(-0.262-0.332 i)\right) \phi_{1 S}+ \\
& \left(g_{1 S}(0.146-0.193 i)+g_{2 S}(0.146+0.193 i)\right) \phi_{2 S}+ \\
& \left(g_{1 S}(0.067-0.255 i)+g_{2 S}(0.067+0.255 i)\right) \phi_{3 S} \\
= & (0.00006+0.00002 i) g_{0 s} \phi_{0 S}+ \\
& \left(g_{1 S}(-0.054-0.276 i)+g_{2 S}(-0.145+0.257 i)\right) \phi_{1 S}+ \\
& \left(g_{1 S}(-0.163-0.043 i)+g_{2 S}(0.269+0.405 i)\right) \phi_{2 S}+ \\
\mathcal{M}_{32} & \left(g_{1 S}(0.166-0.503 i)+g_{2 S}(-0.157-0.077 i)\right) \phi_{3 S}
\end{aligned}
$$

Depending on the particular search $(e, \mu$ or $\tau)$, a displaced vertex might occur. For instance, if one focuses on $\tau$, and if $g_{i S} \ll g_{0 S}$, the constraint on $g_{0 S}<10^{-3}$ (see the above figures) implies that $\mu_{R i}^{M} \rightarrow \tau_{L}+\phi_{k S}$ would have a macroscopic decay length. There are many such cases but it is beyond the scope of this thesis to discuss this issue at length. We merely point out the relationship between the constraints coming from $\mu \rightarrow e \gamma$ and the implication on the search for mirror leptons.

- The other implication concerns the VEV of the singlet Higgs fields. Since the seesaw mechanism implies the masses of the light neutrinos are given by $\sim m_{D}^{2} / M$ and with $M \sim O\left(\Lambda_{E W}\right)$, it was stated in [64] that $m_{D} \sim O(100 \mathrm{keV})$ and that the singlet VEV $\sim O(100 \mathrm{keV})$ if $g_{S} \sim O(1)$. However, constraints from $\mu \rightarrow e \gamma$ imply $g_{0 S}<10^{-3}$ which now brings the singlet VEV up to $O(100 \mathrm{MeV})$. In fact it can even be of the order $O(1 \mathrm{GeV})$. 
From this observation, it is safe to say that there does not appear to be much of a hierarchy problem in the EW-scale $\nu_{R}$ model.

\subsubsection{Summary of the section}

In this chapter, we present an update on a previous analysis 91] for the process $\mu \rightarrow e \gamma$ performed in the original EW-scale $\nu_{R}$ model 64 to an extended model [73]. Mixings effects of neutrinos and charged leptons constructed with a $A_{4}$ symmetry as recently studied in [92] are also taken into account. In this context, the rare process $\mu \rightarrow e \gamma$ is link to interesting new physics beyond the SM in the lepton sector, like neutrino and charged lepton mass mixings, neutrino mass hierarchies, mirror leptons as well as singlet and triplet scalars of $A_{4}$, etc. The related muon anomalous magnetic dipole moment is also studied in detail for the model.

To summarize, we find that

- One can deduce more stringent constraints on the parameter space of the EW-scale $\nu_{R}$ model by using the LFV process $\mu \rightarrow e \gamma$ than the muon anomalous magnetic dipole moment.

- The branching ratio $B(\mu \rightarrow e \gamma)$ shows some sensitivity to the neutrino mass hierarchies in scenario 2 but not scenario 1 , depending on the $A_{4}$ triplet coupling constants. However we are not advocating the use of the process $\mu \rightarrow e \gamma$ to settle the issue of neutrino mass hierarchies. After all, this is a rare process.

- More stringent constraints can be deduced in scenario 1 than scenario 2 using $B(\mu \rightarrow e \gamma)$.

- Future data from MEG experiment with the projected sensitivity will impose further constraints on the parameter space of the model.

- The muon anomalous magnetic dipole moment is sensitive neither to the neutrino mass 
hierarchies nor the scenarios for all 6 cases of the couplings studied here for the model.

Searching for new physics via rare processes is complementary to direct production of new particles at colliders. For $\mu \rightarrow e \gamma$, the relevant new particles in the model are the mirror leptons and scalar singlets running inside the loop diagram. As shown in our analysis, the Yukawa couplings of the Higgs singlets to the leptons in the EW-scale $\nu_{R}$ model are constrained to be small in order to be consistent with the current experimental limit on $B(\mu \rightarrow e \gamma)$. Thus searching for mirror particles of this model at the LHC would be quite interesting since, due to small couplings, they might decay outside the beam pipe and inside the silicon vertex detectors. The $A_{4}$ singlet and triplet scalars are likely to escape detection as missing energies. As an outlook, one would like to generalize this work to $\mu-e$ conversion. This work has been done and will be presented in the following section.

\subsection{Muon-to-Electron Conversion}

Previously on the $\mu \rightarrow e \gamma$ analysis, the most updated limit on $B(\mu \rightarrow e \gamma)$ is from MEG experiment 98

$$
B(\mu \rightarrow e \gamma) \leq 4.2 \times 10^{-13}(90 \% \text { C.L. }) \quad(\text { MEG 2016) }
$$

and its projected improvement 96 is

$$
B(\mu \rightarrow e \gamma) \sim 4 \times 10^{-14}
$$

Recent data from T2K experiment 99 agrees well with the global analysis of neutrino oscillation data 100102 , suggesting that the normal neutrino mass hierarchy $(\mathrm{NH})$ with a $\mathrm{CP}$ violating Dirac phase $\delta_{\mathrm{CP}} \sim 3 \pi / 2$ is slightly preferred. The best fit result for the central values of the PMNS matrix 
elements in the normal neutrino mass hierarchy can be extracted from 100

$$
U_{\mathrm{PMNS}}^{\mathrm{NH}}=\left(\begin{array}{ccc}
0.8251 & 0.5453 & 0.08679+0.1195 i \\
-0.4568+0.0670 i & 0.5854+0.04428 i & 0.6649 \\
0.3171+0.07377 i & -0.5963+0.04875 i & 0.7322
\end{array}\right) .
$$

For the $\mu-e$ conversion in nuclei, the present experimental upper limits on the branching ratios were obtained by SINDRUM II experiment 103,104 for the targets titanium and gold,

$$
\begin{gathered}
B\left(\mu^{-}+\mathrm{Ti} \rightarrow e^{-}+\mathrm{Ti}\right)<4.3 \times 10^{-12}(90 \% \text { C.L. }) \\
B\left(\mu^{-}+\mathrm{Au} \rightarrow e^{-}+\mathrm{Au}\right)<7 \times 10^{-13}(90 \% \text { C.L. })
\end{gathered}
$$

Significant improvements are expected for $\mu-e$ conversion at future experiments like Mu2e at Fermilab in US and COMET at J-PARC in Japan. Projected sensitivities of $\mu-e$ conversion are $105-109$

$$
\begin{aligned}
& B\left(\mu^{-}+\mathrm{Al} \rightarrow e^{-}+\mathrm{Al}\right)<3 \times 10^{-17} \quad(\mathrm{Mu} 2 \mathrm{e}, \mathrm{COMET}) \\
& B\left(\mu^{-}+\mathrm{Ti} \rightarrow e^{-}+\mathrm{Ti}\right)<10^{-18} \quad(\mathrm{Mu} 2 \mathrm{e} I \mathrm{I}, \mathrm{PRISM})
\end{aligned}
$$

A positive signal of any of the above processes (or any process with charged lepton flavor violation $(\mathrm{CLFV}))$ at the current or projected sensitivities of various high intensity experiments would be a clear indication of new physics as well, just like neutrino oscillations. Given the fact that no new physics has showed up yet at the high energy frontier of the Large Hadron Collider (LHC), it is not a surprise that many recent works have been focused on new physics implication of CLFV in the high intensity frontier. For a review on this topics and its possible connection with the muon anomaly, see [110 and references therein.

In section 6.1, we updated a previous calculation 91 for the radiative process $\mu \rightarrow e \gamma$ in the mirror fermion model with electroweak scale non-sterile right-handed neutrinos 64 to an extended version 92 where a horizontal $A_{4}$ symmetry in the lepton sector was imposed. In this section we 
extend this previous analysis 111 to the $\mu-e$ conversion in nuclei, in particular for aluminum, gold and titanium.

An outline of the section is as follows. In section 6.2.1, the calculation of $\mu-e$ conversion in the extended mirror fermion model is presented. In section 6.2.2, we derive a simple relation between the $\mu-e$ conversion rate and the radiative decay rate of $\mu \rightarrow e \gamma$ in the limit of zero momentum transfer and large mirror lepton masses. Numerical results are shown in section6.2.3. To summarize we conduct section 6.2.4. In Appendix A, we briefly review the effective Lagrangian 112, 113 for describing $\mu-e$ conversion; and in Appendix B, we collect some useful formulas used in section 6.2.2.

\subsubsection{Mirror Fermion Model Calculation}

In this section, we compute the effective coupling constants induced at one loop level in the extended model for the $\mu-e$ conversion.

\subsubsection{Photon Contributions and the Monopole and Dipole Form Factors}

In this work we will focus on the contributions from the photon exchange as shown in the Feynman Diagrams of Fig. (6.8). We also compute the contributions from the $Z$-exchange but since they are suppressed by $m_{\mu}^{2} / m_{Z}^{2}$ we will not present them here. The invariant amplitude for $\mu^{-}(p) \rightarrow e^{-}\left(p^{\prime}\right) \gamma^{*}(q)$ with an off-shell photon can be parametrized as

$$
i \mathcal{M}_{\gamma}=-e \bar{u}_{e}\left(p^{\prime}\right) i \Gamma_{\gamma}^{\mu}(q) u_{\mu}(p) A_{\mu}^{*}(q)
$$

where $\Gamma_{\gamma}^{\mu}(q)$ has the following Lorentz and gauge invariant decomposition

$$
\Gamma_{\gamma}^{\mu}(q)=\left(f_{E 0}\left(q^{2}\right)+\gamma_{5} f_{M 0}\left(q^{2}\right)\right)\left(\gamma^{\mu}-\frac{q_{\mu} \phi}{q^{2}}\right)+\left(f_{M 1}\left(q^{2}\right)+\gamma_{5} f_{E 1}\left(q^{2}\right)\right) \frac{i \sigma^{\mu \nu} q_{\nu}}{m_{\mu}} .
$$

The monopole form factors $f_{E 0}, f_{M 0}$ and the dipole form factors $f_{M 1}, f_{E 1}$ can be obtained by generalizing our previous on-shell calculation of $\mu \rightarrow e \gamma$ in the same model [11] to the case of 

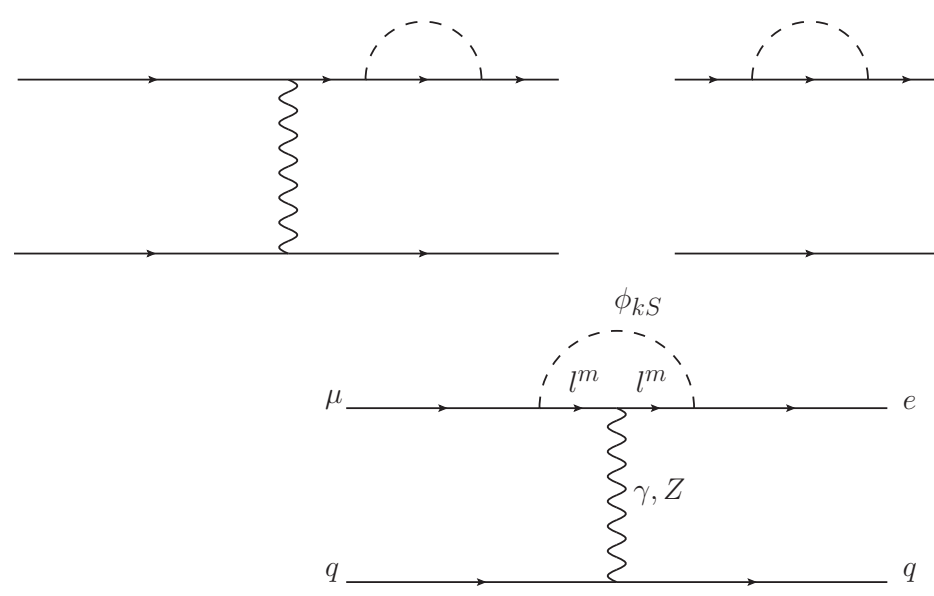

Figure 6.8: One-loop induced Feynman diagrams from photon and $Z$ boson exchanges for $\mu-e$ conversion in electroweak-scale $\nu_{R}$ model.

off-shell photon $\gamma^{*}$. From the Feynman diagrams of Fig. (6.8), we obtain the following expressions

$$
\begin{aligned}
f_{E 0, M 0}\left(q^{2}\right)= & +\frac{1}{32 \pi^{2}} \sum_{k, m} \int_{0}^{1} d x \int_{0}^{1-x} d y\left\{\frac{x y q^{2}}{\Delta_{k m}\left(q^{2}\right)}\left(\mathcal{U}_{1 m}^{L k}\left(\mathcal{U}_{2 m}^{L k}\right)^{*} \pm \mathcal{U}_{1 m}^{R k}\left(\mathcal{U}_{2 m}^{R k}\right)^{*}\right)\right. \\
- & {\left[\log \left(\frac{\Delta_{k m}\left(q^{2}\right)}{\Delta_{k m}(0)}\right)-\left(M_{m}^{2} \pm(1-x-y)^{2} m_{\mu} m_{e}\right)\left(\Delta_{k m}^{-1}\left(q^{2}\right)-\Delta_{k m}^{-1}(0)\right)\right] } \\
& \times\left(\mathcal{U}_{1 m}^{L k}\left(\mathcal{U}_{2 m}^{L k}\right)^{*} \pm \mathcal{U}_{1 m}^{R k}\left(\mathcal{U}_{2 m}^{R k}\right)^{*}\right) \\
+ & (1-x-y)\left(m_{\mu} \pm m_{e}\right) M_{m}\left(\Delta_{k m}^{-1}\left(q^{2}\right)-\Delta_{k m}^{-1}(0)\right) \\
& \left.\times\left(\mathcal{U}_{1 m}^{L k}\left(\mathcal{U}_{2 m}^{R k}\right)^{*} \pm \mathcal{U}_{1 m}^{R k}\left(\mathcal{U}_{2 m}^{L k}\right)^{*}\right)\right\}
\end{aligned}
$$

for the monopole form factors, and

$$
\begin{aligned}
f_{M 1, E 1}\left(q^{2}\right)= & -\frac{m_{\mu}}{32 \pi^{2}} \sum_{k, m} \int_{0}^{1} d x \int_{0}^{1-x} d y \frac{1}{\Delta_{k m}\left(q^{2}\right)} \\
& \times\left\{(1-x-y)\left(y m_{\mu} \pm x m_{e}\right)\left(\mathcal{U}_{1 m}^{L k}\left(\mathcal{U}_{2 m}^{L k}\right)^{*} \pm \mathcal{U}_{1 m}^{R k}\left(\mathcal{U}_{2 m}^{R k}\right)^{*}\right)\right. \\
& \left.+(x+y) M_{m}\left(\mathcal{U}_{1 m}^{L k}\left(\mathcal{U}_{2 m}^{R k}\right)^{*} \pm \mathcal{U}_{1 m}^{R k}\left(\mathcal{U}_{2 m}^{L k}\right)^{*}\right)\right\}
\end{aligned}
$$

for the dipole form factors. Here, we have defined

$$
\Delta_{k m}\left(q^{2}\right)=(x+y) M_{m}^{2}+(1-x-y)\left(m_{k}^{2}-x m_{e}^{2}-y m_{\mu}^{2}\right)-x y q^{2}-i 0^{+},
$$

where $m_{k}$ denotes the mass of scalar singlet $\phi_{k S}$ for $k=0,1,2,3$ and $M_{m}$ the mass of mirror lepton $l_{m}^{M}$ for $m=1,2,3$. 
At $q^{2}=0$, we have $f_{E 0, M 0}(0)=0$ as one would expect. Thus the following reduced monopole form factors $\tilde{f}_{E 0, M 0}$ with an explicit factor of $q^{2}$ extracted from $f_{E 0, M 0}$ are often defined in the literature,

$$
f_{E 0, M 0}\left(q^{2}\right)=\frac{q^{2}}{m_{\mu}^{2}} \tilde{f}_{E 0, M 0}\left(q^{2}\right) .
$$

For small $q^{2}$, one can set $\tilde{f}_{E 0, M 0}\left(q^{2}\right) \approx \tilde{f}_{E 0, M 0}(0)$ with

$$
\begin{gathered}
\tilde{f}_{E 0, M 0}(0)=\frac{m_{\mu}^{2}}{32 \pi^{2}} \sum_{k, m} \int_{0}^{1} d x \int_{0}^{1-x} d y \frac{x y}{\left(\Delta_{k m}(0)\right)^{2}}\left\{\left(\mathcal{U}_{1 m}^{L k}\left(\mathcal{U}_{2 m}^{L k}\right)^{*} \pm \mathcal{U}_{1 m}^{R k}\left(\mathcal{U}_{2 m}^{R k}\right)^{*}\right)\right. \\
\times\left(2 \Delta_{k m}(0)+M_{m}^{2} \pm(1-x-y)^{2} m_{\mu} m_{e}\right) \\
\left.+\left(\mathcal{U}_{1 m}^{L k}\left(\mathcal{U}_{2 m}^{R k}\right)^{*} \pm \mathcal{U}_{1 m}^{R k}\left(\mathcal{U}_{2 m}^{L k}\right)^{*}\right)(1-x-y)\left(m_{\mu} \pm m_{e}\right) M_{m}\right\} .
\end{gathered}
$$

The explicit factor of $q^{2}$ in Eq. 6.43 will cancel the $1 / q^{2}$ of the photon propagator in Fig. 6.8. This leads to four-fermion vector-vector interaction and hence the reduced monopole form factors will contribute to the effective coupling $C_{V(R, L)}^{(q)}$ in the effective Lagrangian of A.1 in Appendix A. We will discuss more about these four-fermion interactions in the next subsection.

At $q^{2}=0$, the contributions from the magnetic and electric dipole terms of Eq. 6.39 to the amplitude $\mathcal{M}_{\gamma}$ in Eq. 6.38 can be reproduced by the following effective Lagrangian

$$
\mathcal{L}_{\gamma, \mathrm{eff}}=\frac{e}{2 m_{\mu}} \bar{e} \sigma^{\alpha \beta}\left(f_{M 1}(0)+\gamma_{5} f_{E 1}(0)\right) \mu F_{\alpha \beta}+\text { H.c. . }
$$

Comparing Eq. (6.45) with the first line of the general form of the Lagrangian for $\mu-e$ conversion given in A.1 in Appendix A, one can deduce the dimensionless effective couplings $C_{D R, D L}$ as linear combinations of the static limit of the dipole form factors $f_{E 1}$ and $f_{M 1}$,

$$
\frac{C_{D R, D L}}{\Lambda^{2}}=\frac{e}{2 m_{\mu}^{2}}\left( \pm f_{E 1}(0)-f_{M 1}(0)\right)
$$




\subsubsection{Four-Fermion Coupling Constants $C_{V(L, R)}^{(q)}$ - Photon Exchange}

The amplitude for $\mu(p) q(k) \rightarrow e\left(p^{\prime}\right) q\left(k^{\prime}\right)$ from the monopole form factors of the photon exchange in Fig. (6.8) can be obtained as

$$
\mathcal{M}_{\gamma}=-e^{2} Q_{q} \overline{u_{e}}\left(p^{\prime}\right)\left(f_{E 0}\left(q^{2}\right)+f_{M 0}\left(q^{2}\right) \gamma_{5}\right)\left(\gamma_{\mu}-\frac{q_{\mu} \phi}{q^{2}}\right) u_{\mu}(p) \frac{1}{q^{2}} \overline{u_{q}}\left(k^{\prime}\right) \gamma^{\mu} u_{q}(k),
$$

where $q=p-p^{\prime}=k^{\prime}-k$, and $f_{E 0}, f_{M 0}$ are given in Eq. 6.40). The $q_{\mu}$ term in (6.47) can be dropped due to quark current conservation. As mentioned earlier, the $1 / q^{2}$ of the photon propagator will be cancelled from a factor of $q^{2}$ in $f_{E 0, M 0}$. Thus in terms of the reduced form factors $\tilde{f}_{E 0, M 0}$ of Eq. 6.43), the amplitude $\mathcal{M}_{\gamma}$ can be rewritten as

$$
\begin{aligned}
& \mathcal{M}_{\gamma}=-\frac{e^{2} Q_{q}}{m_{\mu}^{2}} {\left[\left(\tilde{f}_{E 0}-\tilde{f}_{M 0}\right) \overline{u_{L e}}\left(p^{\prime}\right) \gamma_{\mu} u_{L \mu}(p)+\left(\tilde{f}_{E 0}+\tilde{f}_{M 0}\right) \overline{u_{R e}}\left(p^{\prime}\right) \gamma_{\mu} u_{R \mu}(p)\right] } \\
& \times\left[\overline{u_{L q}}\left(k^{\prime}\right) \gamma^{\mu} u_{L q}(k)+\overline{u_{R q}}\left(k^{\prime}\right) \gamma^{\mu} u_{R q}(k)\right]
\end{aligned}
$$

where $\tilde{f}_{E 0, M 0}$ are defined in Eq. 6.44 for small $q^{2}$. At $q^{2}=0$, this amplitude can be reproduced by the following Fermi interaction

$$
\begin{aligned}
& \mathcal{L}_{\gamma, \mathrm{eff}}^{\prime}=-\frac{e^{2} Q_{q}}{m_{\mu}^{2}} {\left[\left(\tilde{f}_{E 0}(0)-\tilde{f}_{M 0}(0)\right) \overline{e_{L}} \gamma_{\mu} \mu_{L}+\left(\tilde{f}_{E 0}(0)+\tilde{f}_{M 0}(0)\right) \overline{e_{R}} \gamma_{\mu} \mu_{R}\right] } \\
& \times\left[\bar{q} \gamma^{\mu} q\right] .
\end{aligned}
$$

By matching Eq. (6.49) with the second line of the general form of the Lagrangian for $\mu-e$ conversion given in A.1 in Appendix A, we deduce the following relations for the dimensionless effective couplings $C_{V(L, R)}^{(q) \gamma}$

$$
\frac{C_{V(L, R)}^{(q) \gamma}}{\Lambda^{2}}=\frac{e^{2} Q_{q}}{m_{\mu}^{2}}\left(\tilde{f}_{E 0}(0) \mp \tilde{f}_{M 0}(0)\right) .
$$

Note that we have the relation $C_{V(L, R)}^{(u) \gamma}=-2 C_{V(L, R)}^{(d) \gamma}$. This implies the vector effective couplings $\tilde{C}_{V(L, R)}^{(n) \gamma}$ for the neutron from the photon exchange are vanishing. This is expected since neutron carries no electric charge.

We also note that for the photon contributions, only $C_{V R}^{(q)}$ and $C_{V L}^{(q)}$ are non-vanishing. Other four-fermion effective couplings will be non-vanishing only from $Z$ exchange, scalar exchange or 
box diagrams, which are negligible as compared with the photon exchange contributions. We prove this in Appendix C.

\subsubsection{The relationship between $\mu-e$ Conversion and $\mu \rightarrow e \gamma$}

Since the momentum transfer $q^{2}$ is expected to be quite small in the $\mu-e$ conversion process in nuclei, we can make a Taylor expansion for the various form factors deduced in the previous section around $q^{2}=0$. Thus for small $q^{2}$, we have

$$
\begin{aligned}
f_{E 0, M 0}\left(q^{2}\right) \approx \frac{q^{2}}{32 \pi^{2}} \frac{1}{M_{m}^{4}} \sum_{k, m}\{ & \left(\mathcal{U}_{1 m}^{L k}\left(\mathcal{U}_{2 m}^{L k}\right)^{*} \pm \mathcal{U}_{1 m}^{R k}\left(\mathcal{U}_{2 m}^{R k}\right)^{*}\right) \\
& \times\left[M_{m}^{2}\left(\mathcal{I}\left(r_{k m}\right)+2 \mathcal{I}_{30}\left(r_{k m}\right)\right) \pm m_{\mu} m_{e} \mathcal{I}_{10}\left(r_{k m}\right)\right] \\
+ & \left.\left(\mathcal{U}_{1 m}^{L k}\left(\mathcal{U}_{2 m}^{R k}\right)^{*} \pm \mathcal{U}_{1 m}^{R k}\left(\mathcal{U}_{2 m}^{L k}\right)^{*}\right) M_{m}\left(m_{\mu} \pm m_{e}\right) \mathcal{I}_{20}\left(r_{k m}\right)\right\}
\end{aligned}
$$

and

$$
\begin{gathered}
f_{M 1, E 1}\left(q^{2}\right) \approx-\frac{m_{\mu}}{32 \pi^{2}} \sum_{k, m}\left\{\frac{1}{M_{m}^{2}}\left(m_{\mu} \pm m_{e}\right)\left(\mathcal{U}_{1 m}^{L k}\left(\mathcal{U}_{2 m}^{L k}\right)^{*} \pm \mathcal{U}_{1 m}^{R k}\left(\mathcal{U}_{2 m}^{R k}\right)^{*}\right) \mathcal{I}\left(r_{k m}\right)\right. \\
\left.\quad+\frac{1}{M_{m}}\left(\mathcal{U}_{1 m}^{L k}\left(\mathcal{U}_{2 m}^{R k}\right)^{*} \pm \mathcal{U}_{1 m}^{R k}\left(\mathcal{U}_{2 m}^{L k}\right)^{*}\right) \mathcal{J}\left(r_{k m}\right)\right\} \\
-\frac{m_{\mu} q^{2}}{32 \pi^{2}}\left\{\frac{1}{M_{m}^{4}}\left(m_{\mu} \pm m_{e}\right)\left(\mathcal{U}_{1 m}^{L k}\left(\mathcal{U}_{2 m}^{L k}\right)^{*} \pm \mathcal{U}_{1 m}^{R k}\left(\mathcal{U}_{2 m}^{R k}\right)^{*}\right) \mathcal{I}_{40}\left(r_{k m}\right)\right. \\
\left.+\frac{1}{M_{m}^{3}}\left(\mathcal{U}_{1 m}^{L k}\left(\mathcal{U}_{2 m}^{R k}\right)^{*} \pm \mathcal{U}_{1 m}^{R k}\left(\mathcal{U}_{2 m}^{L k}\right)^{*}\right) \mathcal{I}_{50}\left(r_{k m}\right)\right\} .
\end{gathered}
$$

Here $r_{k m}=m_{k}^{2} / M_{m}^{2}$ and the expressions for the Feynman parameterization integrals $\mathcal{I}, \mathcal{J}$ and $\mathcal{I}_{i 0}(i=1,2, \cdots, 5)$ can be found in Appendix B.

From A.2 in Appendix A, the conversion rate (for $\gamma$ exchange) is given by

$$
\Gamma_{\text {conv }}=\frac{m_{\mu}^{5}}{4 \Lambda^{4}}\left(\left|C_{D R} D+4 \tilde{C}_{V R}^{(p)} V^{(p)}\right|^{2}+\left|C_{D L} D+4 \tilde{C}_{V L}^{(p)} V^{(p)}\right|^{2}\right),
$$

where $C_{D R, D L}$ is given by 6.46 , and $\tilde{C}_{V R, V L}^{(p)}$ are given by A.3 and A.5 in Appendix A. To obtain (6.53), we have used the following result valid for the neutron,

$$
\tilde{C}_{V(L, R)}^{(n)}=\sum_{u, d, s} C_{V(L, R)}^{(q)} f_{V n}^{(q)}=0
$$


Using the above approximate form factors $(6.51)$ and 6.52 for small $q^{2}$, we can derive

$$
\begin{aligned}
C_{D R, D L} \approx \frac{e \Lambda^{2}}{32 \pi^{2} m_{\mu}} \sum_{k, m} & \left\{\frac{\mathcal{I}\left(r_{k m}\right)}{M_{m}^{2}}\left(m_{\mu} \mathcal{U}_{1 m}^{R, L k}\left(\mathcal{U}_{2 m}^{R, L k}\right)^{*}+m_{e} \mathcal{U}_{1 m}^{L, R k}\left(\mathcal{U}_{2 m}^{L, R k}\right)^{*}\right)\right. \\
+ & \frac{\mathcal{J}\left(r_{k m}\right)}{M_{m}} \mathcal{U}_{1 m}^{R, L k}\left(\mathcal{U}_{2 m}^{L, R k}\right)^{*} \\
+ & \frac{q^{2}}{M_{m}^{2}}\left[\frac{\mathcal{I}_{40}\left(r_{k m}\right)}{M_{m}^{2}}\left(m_{\mu} \mathcal{U}_{1 m}^{R, L k}\left(\mathcal{U}_{2 m}^{R, L k}\right)^{*}+m_{e} \mathcal{U}_{1 m}^{L, R k}\left(\mathcal{U}_{2 m}^{L, R k}\right)^{*}\right)\right. \\
& \left.\left.+\frac{\mathcal{I}_{50}\left(r_{k m}\right)}{M_{m}} \mathcal{U}_{1 m}^{R, L k}\left(\mathcal{U}_{2 m}^{L, R k}\right)^{*}\right]\right\},
\end{aligned}
$$

and summing over the contributions from light quarks, we have

$$
\begin{aligned}
& \tilde{C}_{V L, V R}^{(p)} \approx \frac{e^{2} \Lambda^{2}}{16 \pi^{2} M_{m}^{4}} \sum_{k, m}\left\{M_{m}^{2}\left(\mathcal{I}\left(r_{k m}\right)+2 \mathcal{I}_{30}\left(r_{k m}\right)\right) \mathcal{U}_{1 m}^{R, L k}\left(\mathcal{U}_{2 m}^{R, L k}\right)^{*}\right. \\
& +m_{\mu} m_{e} \mathcal{I}_{10}\left(r_{k m}\right) \mathcal{U}_{1 m}^{L, R k}\left(\mathcal{U}_{2 m}^{L, R k}\right)^{*} \\
& \left.+M_{m} \mathcal{I}_{20}\left(r_{k m}\right)\left(m_{\mu} \mathcal{U}_{1 m}^{R, L k}\left(\mathcal{U}_{2 m}^{L, R k}\right)^{*}+m_{e} \mathcal{U}_{1 m}^{L, R k}\left(\mathcal{U}_{2 m}^{R, L k}\right)^{*}\right)\right\} \text {. }
\end{aligned}
$$

Dropping the $q^{2}$ terms in $C_{D R, D L}$ and keeping only those terms up to $\mathcal{O}\left(1 / M_{m}^{2}\right)$ in $\tilde{C}_{V L, V R}^{(p)}$, we obtain for the conversion rate

$$
\begin{aligned}
\Gamma_{\text {conv }}\left(q^{2} \rightarrow 0\right) \approx & \frac{m_{\mu}^{5}}{4} \frac{1}{\left(32 \pi^{2}\right)^{2}} \\
\times & \sum_{k, m}\left\{\left|\frac{16 \pi^{2} D}{m_{\mu}} C_{L}^{k m}+8 V^{(p)} e^{2} \frac{\mathcal{I}\left(r_{k m}\right)+2 \mathcal{I}_{30}\left(r_{k m}\right)}{M_{m}^{2}} \mathcal{U}_{1 m}^{L k}\left(\mathcal{U}_{2 m}^{L k}\right)^{*}\right|^{2}\right. \\
& \left.+\left|\frac{16 \pi^{2} D}{m_{\mu}} C_{R}^{k m}+8 V^{(p)} e^{2} \frac{\mathcal{I}\left(r_{k m}\right)+2 \mathcal{I}_{30}\left(r_{k m}\right)}{M_{m}^{2}} \mathcal{U}_{1 m}^{R k}\left(\mathcal{U}_{2 m}^{R k}\right)^{*}\right|^{2}\right\}
\end{aligned}
$$

where

$$
\begin{aligned}
C_{L, R}^{k m}=\frac{e}{16 \pi^{2}}\{ & \frac{\mathcal{I}\left(r_{k m}\right)}{M_{m}^{2}}\left(m_{\mu} \mathcal{U}_{1 m}^{R, L k}\left(\mathcal{U}_{2 m}^{R, L k}\right)^{*}+m_{e} \mathcal{U}_{1 m}^{L, R k}\left(\mathcal{U}_{2 m}^{L, R k}\right)^{*}\right) \\
& \left.+\frac{\mathcal{J}\left(r_{k m}\right)}{M_{m}} \mathcal{U}_{1 m}^{R, L k}\left(\mathcal{U}_{2 m}^{L, R k}\right)^{*}\right\} .
\end{aligned}
$$

Recall that for the on-shell process $\mu \rightarrow e \gamma$, we have

$$
\Gamma_{\mu \rightarrow e \gamma}=\frac{1}{16 \pi} m_{\mu}^{3} \sum_{k, m}\left(\left|C_{L}^{k m}\right|^{2}+\left|C_{R}^{k m}\right|^{2}\right) .
$$


Thus, one obtains

$$
\begin{gathered}
\Gamma_{\text {conv }}\left(q^{2} \rightarrow 0\right) \approx \pi D^{2} \Gamma_{\mu \rightarrow e \gamma}+\frac{m_{\mu}^{5}}{\left(64 \pi^{2}\right)^{2}} \sum_{k, m}\left\{2 D V^{(p)}(8 \pi e)^{2} \frac{\mathcal{I}\left(r_{k m}\right)+2 \mathcal{I}_{30}\left(r_{k m}\right)}{m_{\mu} M_{m}^{2}}\right. \\
\times\left(C_{L}^{k m} \mathcal{U}_{1 m}^{L k}\left(\mathcal{U}_{2 m}^{L k}\right)^{*}+\left(C_{L}^{k m}\right)^{*}\left(\mathcal{U}_{1 m}^{L k}\right)^{*} \mathcal{U}_{2 m}^{L k}\right. \\
\left.+C_{R}^{k m} \mathcal{U}_{1 m}^{R k}\left(\mathcal{U}_{2 m}^{R k}\right)^{*}+\left(C_{R}^{k m}\right)^{*}\left(\mathcal{U}_{1 m}^{R k}\right)^{*} \mathcal{U}_{2 m}^{R k}\right) \\
\left.+\left(8 V^{(p)} e^{2} \frac{\mathcal{I}\left(r_{k m}\right)+2 \mathcal{I}_{30}\left(r_{k m}\right)}{M_{m}^{2}}\right)^{2}\left(\left|\mathcal{U}_{1 m}^{L k}\left(\mathcal{U}_{2 m}^{L k}\right)^{*}\right|^{2}+\left|\mathcal{U}_{1 m}^{R k}\left(\mathcal{U}_{2 m}^{R k}\right)^{*}\right|^{2}\right)\right\} .
\end{gathered}
$$

Note that since $C_{L, R}^{k m}$ is scaled by $1 / M_{m}$, the second and the third terms in 6.60 are suppressed by $1 / M_{m}$ and $1 / M_{m}^{2}$ respectively, as compared with the first term. If one drops these two suppressed terms further, one obtains a simple relation

$$
\Gamma_{\text {conv }}\left(q^{2} \rightarrow 0\right) \approx \pi D^{2} \Gamma_{\mu \rightarrow e \gamma} .
$$

Thus,

$$
B_{\mu N \rightarrow e N}=\frac{\Gamma_{\text {conv }}}{\Gamma_{\text {capt }}} \approx \pi D^{2} \frac{\Gamma_{\mu}}{\Gamma_{\text {capt }}} B_{\mu \rightarrow e \gamma}
$$

where $\Gamma_{\mu}$ is the total decay width of the muon.

\subsubsection{Numerical analysis}

In our analysis, we adapt the same assumptions for the parameter space as was done in 111 and presented in section 6.1.3. We summarize them as follows.

- For the mass parameters, we take the masses of the singlet scalars $\phi_{k S}$ to be

$$
m_{0}: m_{1}: m_{2}: m_{3}=M_{S}: 2 M_{S}: 3 M_{S}: 4 M_{S},
$$

where the common mass $M_{S}$ is set to be $10 \mathrm{MeV}$; and for the mirror lepton masses, we set

$$
M_{m}=M_{\text {mirror }}+\delta_{m}
$$

where $\delta_{1}=0, \delta_{2}=10 \mathrm{GeV}, \delta_{3}=20 \mathrm{GeV}$ and the common mass $M_{\text {mirror }}$ is varied in the range of $100-800 \mathrm{GeV}$. Our results are insensitive to these choices as long as $m_{k} / M_{m} \ll 1$. 
- Note that the relations $g_{2 S}=\left(g_{1 S}\right)^{*}$ and $g_{2 S}^{\prime}=\left(g_{1 S}^{\prime}\right)^{*}$ hold due to the hermiticity of the neutrino Dirac mass matrix. However, all the Yukawa couplings $g_{0 S}, g_{1 S}, g_{2 S}, g_{0 S}^{\prime}, g_{1 S}^{\prime}$, and $g_{2 S}^{\prime}$ are assumed to be real.

- Out of the four mixing matrices, only the one $U_{\mathrm{PMNS}}$ associated with the left-handed SM fermions are known. Following [111, we will consider two scenarios below:

* Scenario 1: $U_{\mathrm{PMNS}}^{M}=U_{\mathrm{PMNS}}^{\prime}=U_{\mathrm{PMNS}}^{\prime M}=U_{\mathrm{CW}}^{\dagger}$

* Scenario 2: $U_{\mathrm{PMNS}}^{M}=U_{\mathrm{PMNS}}^{\prime}=U_{\mathrm{PMNS}}^{\prime M}=U_{\mathrm{PMNS}}$

where $U_{\mathrm{CW}}$ is given by (5.6). For the PMNS mixing matrix, we will use the best fit result in (6.33). In the two scenarios that we are studying, our results do not depend sensitively on the mass hierarchies.

- We will study the following two cases for the Yukawa couplings.

(1) $g_{0 S}=g_{0 S}^{\prime}$ and $g_{1 S}=g_{1 S}^{\prime}=10^{-2} g_{0 S}$. Hence the contributions from the $A_{4}$ triplet is small.

(2) $g_{0 S}=g_{0 S}^{\prime}=g_{1 S}=g_{1 S}^{\prime}$. Both $A_{4}$ singlet and triplet terms carry the same weight.

In Figs. 6.9), 6.10), 6.11) and 6.12 we plot the contour of $\log _{10} B(\mu-e$ conversion) with $\gamma$ dominance in the $\left(\log _{10}\left(g_{0 S}\right), M_{\text {mirror }}\right)$ plane for Scenarios 1 and 2 with the normal neutrino mass hierarchy for the 2 cases of couplings aforementioned respectively. The blue and green solid lines correspond to the current limits from SINDRUM II experiments for $\mu-e$ conversion to titanium (6.34) and gold (6.35) respectively. The red solid and dashed lines correspond to the current limit 6.31 and projected sensitivity 6.32 for $\mu \rightarrow e \gamma$ from MEG experiment. The cyan and blue dashed lines correspond to the projected sensitivities for $\mu-e$ conversion to aluminum and titanium from COMET, Mu2e (6.36) and Mu2e II, PRISM 6.37) experiments respectively.

Several comments are in order here. 


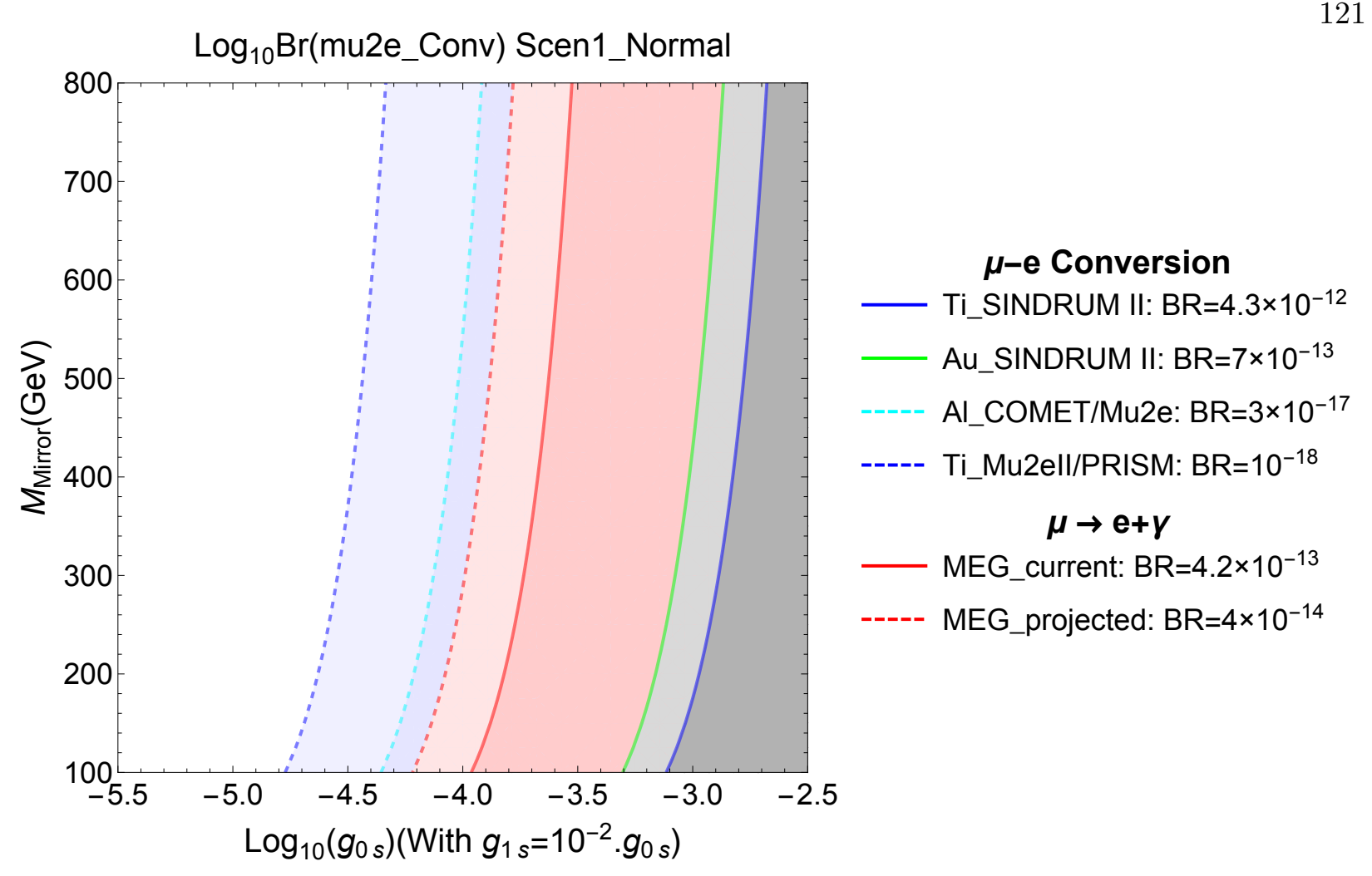

Figure 6.9: Contour plots of $\log _{10} B(\mu-e$ conversion $)$ on the $\left(g_{0 S}, M_{\text {mirror }}\right)$ plane for normal mass hierarchy in Scenario 1 with $g_{0 S}=g_{0 S}^{\prime}$ and $g_{1 S}=g_{1 S}^{\prime}=10^{-2} g_{0 S}$. The legend shows current experimental limits and projected sensitivities from COMET, Mu2e, SINDRUM II, PRISM and MEG. For details of other input parameters, one can refer to the text in section 6.2 .3

- We have studied in some details the effects of different settings of couplings on our results. Generally, we observe that as one varies the $A_{4}$ triplet coupling $g_{1 S}$ from $10^{-2} g_{0 S}$ to $g_{0 S}$ (from Figs. 6.9 to 6.12) the contour plots for $\log _{10} B(\mu-e$ conversion) are shifted to the left. The $A_{4}$ triplet is playing a significant role in putting constraints on the parameter space for the CLFV processes, such as $\mu \rightarrow e \gamma$ and $\mu-e$ conversion in the model.

- For the sensitivity of the two scenarios, we find that

* Generally, Scenario 2 is less stringent constraint than Scenario 1.

* In particular, when the $A_{4}$ singlet couplings are dominated, Scenario 2 is less stringent than Scenario 1 by at least two order of magnitude $\left(10^{-3}\right.$ vs. $\left.10^{-1}\right)$, regarding the constraint on the couplings (as shown in Figs. 6.9) and (6.10). This is due to the 


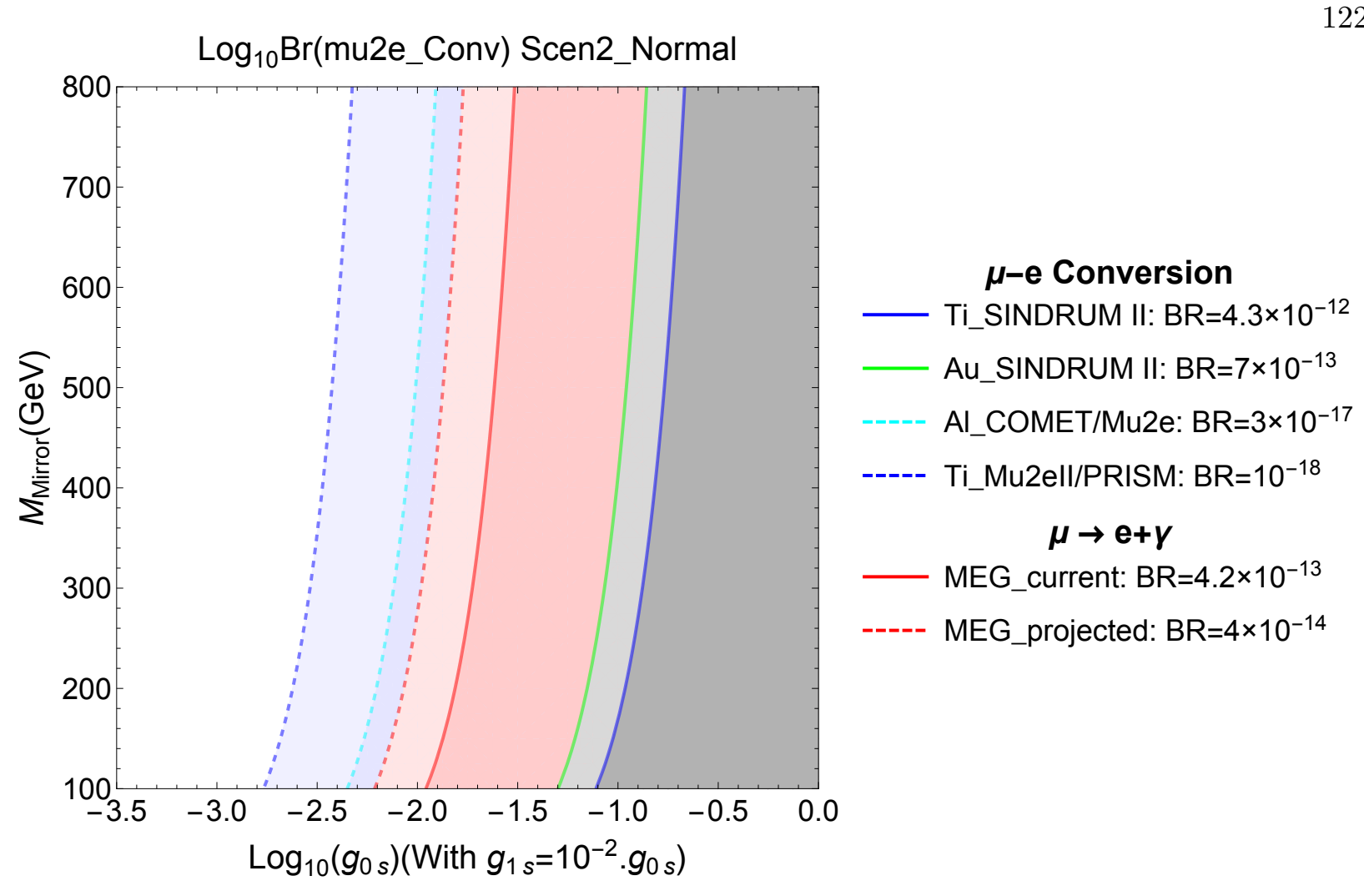

Figure 6.10: Contour plots of $\log _{10} B(\mu-e$ conversion $)$ on the $\left(g_{0 S}, M_{\text {mirror }}\right)$ plane for normal mass hierarchy in Scenarios 2 with $g_{0 S}=g_{0 S}^{\prime}$ and $g_{1 S}=g_{1 S}^{\prime}=10^{-2} g_{0 S}$.

fact that in Scenario 2, the three unknown unitary mixing matrices are now departure from $U_{\text {PMNS }}$ which allows for larger effects since the amplitudes involve products of both the couplings and the elements of mixing matrices.

* However, as one turns on the contribution from the $A_{4}$ triplet in Fig. (6.11) and Fig. 6.12, the discrepancy between two scenarios 1 and 2 shrink $\left(10^{-2}\right.$ vs. $\left.10^{-3.2}\right)$. It implies that Scenario 2 is more sensitive to the change in the structure of $A_{4}$ couplings.

- Finally, regarding the incorporation of the current limit on $B(\mu \rightarrow e \gamma)$ from MEG experiment and its projected sensitivity into the contour plots of $\log _{10} B(\mu-e$ conversion), one can obtain the following statements by looking at Figs. 6.9)- 6.12):

* The plots illustrate nicely the close relation between the two CLFV processes $\mu \rightarrow e \gamma$ 


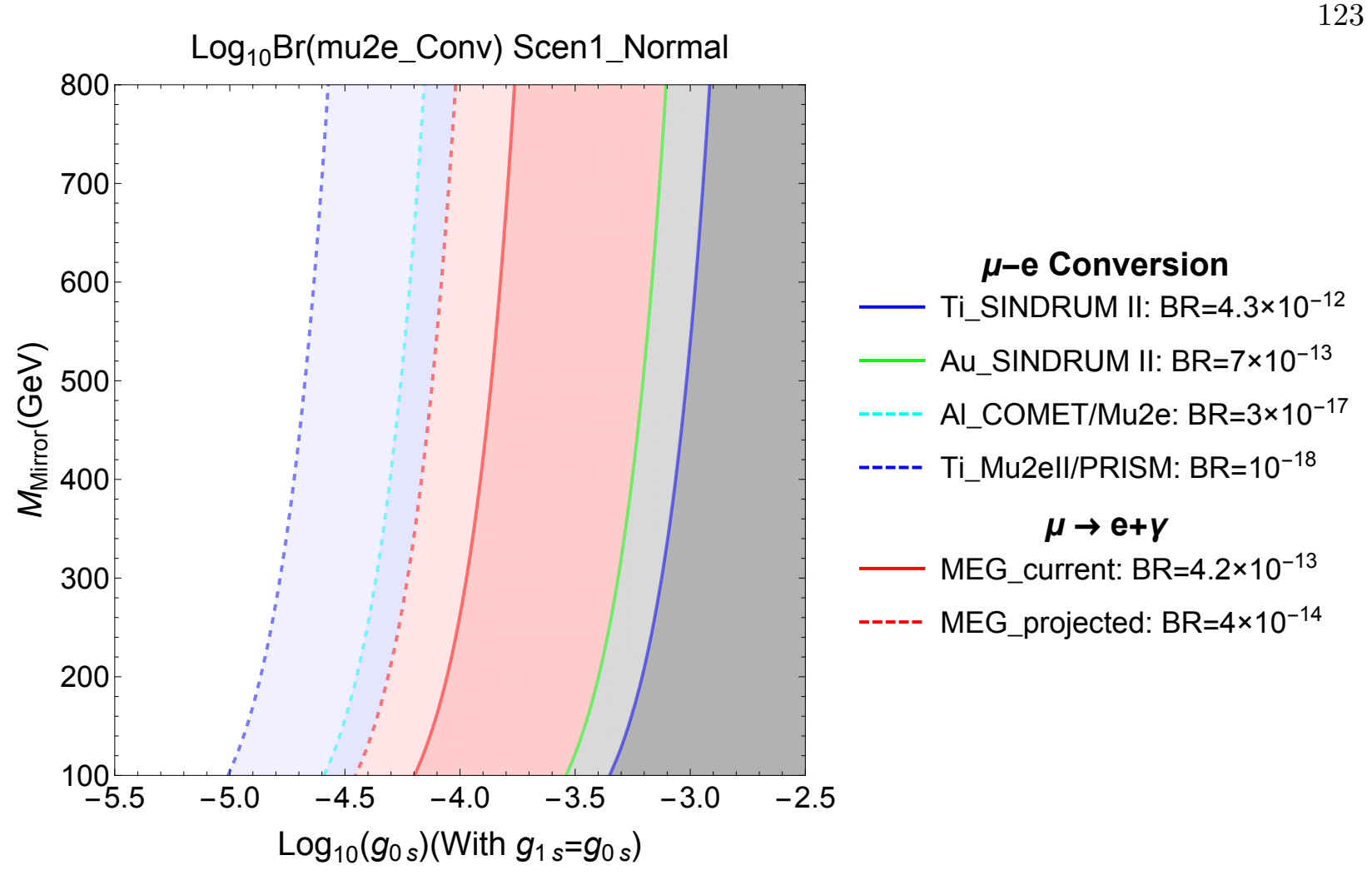

Figure 6.11: Contour plots of $\log _{10} B(\mu-e$ conversion $)$ on the $\left(g_{0 S}, M_{\text {mirror }}\right)$ plane for normal mass hierarchy in Scenarios 1 with $g_{0 S}=g_{0 S}^{\prime}=g_{1 S}=g_{1 S}^{\prime}$.

and $\mu-e$ conversion in nuclei using the simple formula 6.62 we derived in Sec. 6.2 .2

$*$ In the same parameter space, $\mu \rightarrow e \gamma$ shows a tighter constraint than $\mu-e$ conversion by the fact that it excludes almost half of the searched region for the branching ratio of $\mu-e$ conversion. Therefore, our work helps narrow down future searches for $\mu-e$ conversion at Fermilab/Mu2e, J-PARC/COMET and PRISM.

* With the current upper bounds from various experiments, the radiative decay $\mu \rightarrow e \gamma$ is providing more stringent constraints on the couplings than the $\mu-e$ conversion $\left(10^{-4}\right.$ vs. $10^{-3}$, about one order of magnitude better). However, for the future projected sensitivities at Mu2e and COMET, $\mu-e$ conversion is slightly more stringent, about half an order of magnitude stronger constraints on the couplings. For PRISM, it can be about an order of magnitude more stronger. 


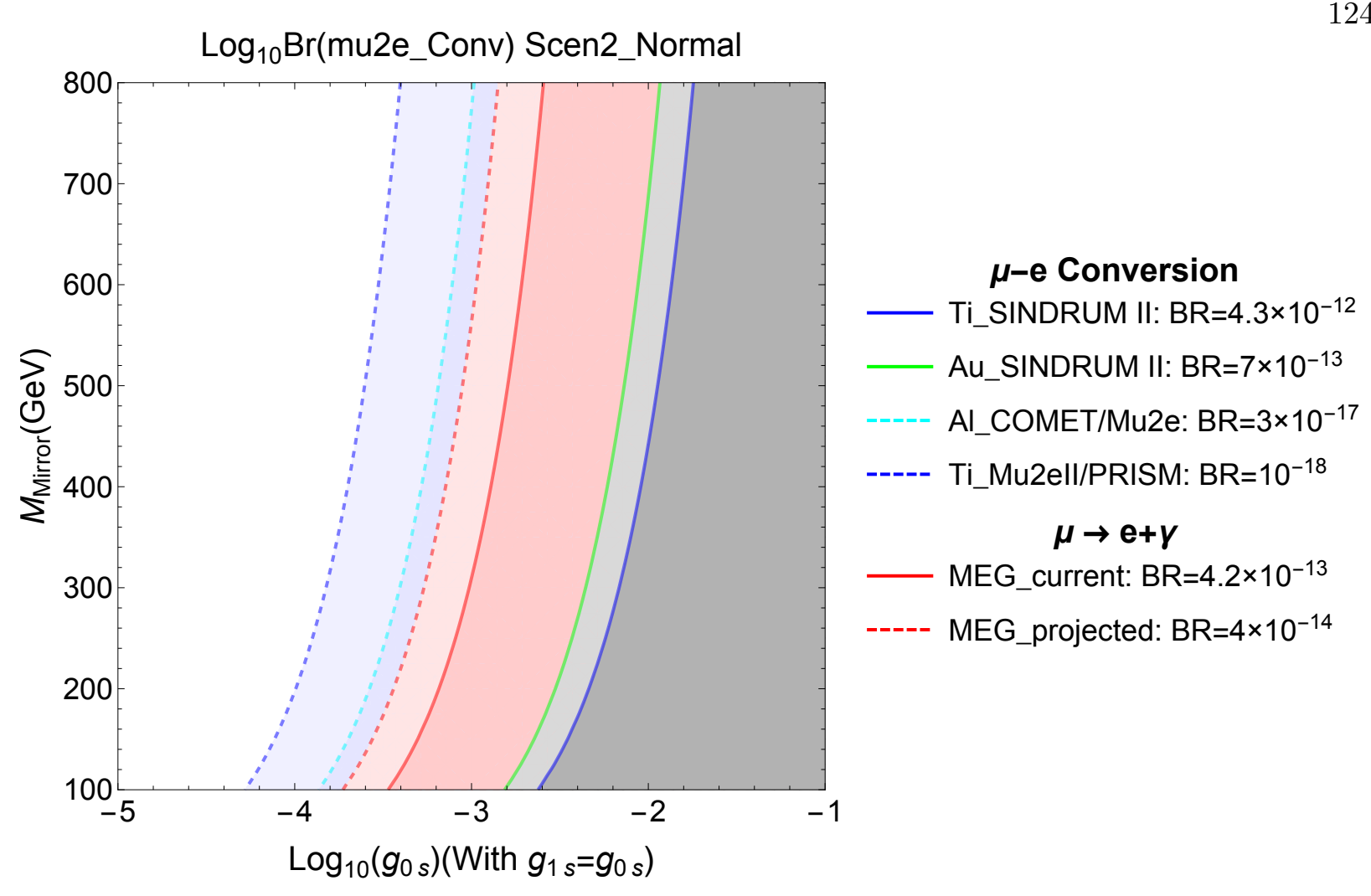

Figure 6.12: Contour plots of $\log _{10} B(\mu-e$ conversion $)$ on the $\left(g_{0 S}, M_{\text {mirror }}\right)$ plane for normal mass hierarchy in Scenarios 2 with $g_{0 S}=g_{0 S}^{\prime}=g_{1 S}=g_{1 S}^{\prime}$.

\subsubsection{Summary of the section}

Mirror fermion model with electroweak scale non-sterile right-handed neutrinos is an interesting extension of the SM. Aside from its aesthetically appealing to restoring parity symmetry at higher energy scale, it can have immediate impacts for experiments in both complementary frontiers of high energy and high intensity searching for new physics of lepton flavor violation.

In this study, we discussed $\mu-e$ conversion in nuclei and radiative decay $\mu \rightarrow e \gamma$ in an extended mirror fermion model with a $A_{4}$ horizontal symmetry in the lepton sector. Currently the most stringent constraint on the parameter space of the model is provided by the most recent limit on the radiative decay $\mu \rightarrow e \gamma$ from MEG. In the future, Mu2e and COMET experiments can provide more stringent constraints on the model from $\mu-e$ conversion in aluminum. The sensi- 
tivity of the new Yukawa couplings can be probed is of order $10^{-5}$, about one order of magnitude improvement compared with current status from MEG. Small Yukawa couplings such as $10^{-5}$ can give rise to distinct signatures in the search of mirror charged leptons and Majorana right-handed neutrinos at the LHC (or planned colliders) in the form of displaced decay vertices with decay lengths larger than $1 \mathrm{~mm}$ or so [119]. Although unrelated to the present analysis, a similar remark can be made for the search for mirror quarks $[74]$. 


\section{Chapter 7}

\section{Implications on Quark Mass Matrices}

\subsection{Motivation}

Quark mass terms come from Yukawa interactions with the Higgs doublet but the SM cannot predict the couplings. As far as we have known quark masses have been determined by experiments. Moreover quark sector shows a very distinctive mass hierarchy in which $m_{t} / m_{u} \sim \mathcal{O}\left(10^{5}\right)$ for the up-type quarks and $m_{b} / m_{d} \sim \mathcal{O}\left(10^{3}\right)$ for the down-type quarks. These facts provide us freedom to build a model of quark masses in order to reasonably explain the origin of quark masses and their mass structure.

As discussed in chapter 5, the model of neutrino masses and mixings eventually necessitated a slight breaking of the discrete $A_{4}$ symmetry in order to avoid the case of degenerate charged lepton from occurring. This led to the ansätz for $U_{l L}$ which has deviated from the unit matrix by a small amount and still been unitary. Therefore the Wolfenstein-like parametrized form has been proposed

$$
U_{l L}=\left(\begin{array}{ccc}
1-\frac{\lambda_{l}^{2}}{2} & \lambda_{l} & A_{l} \lambda_{l}^{3}\left(\rho_{l}-i \eta_{l}\right) \\
-\lambda_{l} & 1-\frac{\lambda_{l}^{2}}{2} & A_{l} \lambda_{l}^{2} \\
A_{l} \lambda_{l}^{3}\left(1-\rho_{l}-i \eta_{l}\right) & -A_{l} \lambda_{l}^{2} & 1
\end{array}\right)
$$

where $A_{l}, \rho_{l}, \eta_{l}$ are real parameters of $\mathrm{O}(1)[22$. The subscript ' $l$ ' indicates that $A, \rho, \eta$ belong to charged leptons. 
On the other hand both quarks and charged leptons acquire their masses through the couplings to the SM Higgs doublet $\Phi_{2}$. It might not be unreasonable to speculate that whatever mechanism giving rise to mass mixings in mass matrices could be similar for both quarks and charged leptons. The following ansätz could be made for quark sector

$$
\begin{aligned}
U_{l L} & \rightarrow U_{d L} \\
\lambda_{l}, A_{l}, \rho_{l}, \eta_{l} & \rightarrow \lambda_{d}, A_{d}, \rho_{d}, \eta_{d} \\
U_{l L} & \rightarrow U_{u L} \\
\lambda_{l}, A_{l}, \rho_{l}, \eta_{l} & \rightarrow \lambda_{u}, A_{u}, \rho_{u}, \eta_{u}
\end{aligned}
$$

With the current knowledge about the CKM matrix, defined as $V_{C K M}=U_{u L}^{\dagger} U_{d L}$, and the absolute quark masses $m_{u}, m_{d}, \cdots m_{b}$ one can have the forms of $\mathcal{M}_{u} \mathcal{M}_{u}^{\dagger}$ and $\mathcal{M}_{d} \mathcal{M}_{d}^{\dagger}$ being similar to that of $\mathcal{M}_{l} \mathcal{M}_{l}^{\dagger}$. From this we propose quark mass matrix forms and use the mentioned knowledge to constrain their parameters.

The chapter is organized as follows. Firstly, we find a relation between $\lambda_{C K M}$ (or $\lambda$ for short), $\lambda_{u}$ and $\lambda_{d}$. This will serve as our base for any assumption we have made in this chapter. Secondly, we present a possible quark mass matrix form and with a general checking on its eigenvalues we move forward to construct $\mathcal{M}_{u}$ and $\mathcal{M}_{d}$ by using the resulting $\mathcal{M}_{u, d} \mathcal{M}_{u, d}^{\dagger}$ to constrain the parameters of up- and down-quark mass matrices. We also include numerical analysis to make sure that these matrices are coherent with our current understanding about the quark sector.

\subsection{Relation between $\lambda, \lambda_{u}$ and $\lambda_{d}$}

As argued in the previous section and in 92 we can reuse the Wolfenstein-like parameterization method and apply it for $U_{d L}$ and $U_{u L}$. The resulting mixing matrices have the following 
forms

$$
\begin{gathered}
U_{d L}=\left(\begin{array}{ccc}
1-\frac{\lambda_{d}^{2}}{2} & \lambda_{d} & A_{d} \lambda_{d}^{3}\left(\rho_{d}-i \eta_{d}\right) \\
-\lambda_{d} & 1-\frac{\lambda_{d}^{2}}{2} & A_{d} \lambda_{d}^{2} \\
A_{d} \lambda_{d}^{3}\left(1-\rho_{d}-i \eta_{d}\right) & -A_{d} \lambda_{d}^{2} & 1
\end{array}\right) \\
U_{u L}=\left(\begin{array}{ccc}
1-\frac{\lambda_{u}^{2}}{2} & \lambda_{u} & A_{u} \lambda_{u}^{3}\left(\rho_{u}-i \eta_{u}\right) \\
-\lambda_{u} & 1-\frac{\lambda_{u}^{2}}{2} & A_{u} \lambda_{u}^{2} \\
A_{u} \lambda_{u}^{3}\left(1-\rho_{u}-i \eta_{u}\right) & -A_{u} \lambda_{u}^{2} & 1
\end{array}\right)
\end{gathered}
$$

Recall that

$$
V_{C K M}=U_{u L}^{\dagger} U_{d L}
$$

It is well-known that this CKM matrix [18, 19] can also be parametrized by three mixing angles and the $C P$-violation phase [19]. The standard expression has been mentioned in Eq. 2.105). It is convenient to write the matrix in terms of Wolfenstein parametrization as

$$
V_{C K M}=\left(\begin{array}{ccc}
V_{11} & V_{12} & V_{13} \\
V_{21} & V_{22} & V_{23} \\
V_{31} & V_{32} & V_{33}
\end{array}\right)=\left(\begin{array}{ccc}
1-\frac{\lambda^{2}}{2} & \lambda & A \lambda^{3}(\rho-i \eta) \\
-\lambda & 1-\frac{\lambda^{2}}{2} & A \lambda^{2} \\
A \lambda^{3}(1-\rho-i \eta) & -A \lambda^{2} & 1
\end{array}\right)
$$

Where $\lambda=0.22496 \pm 0.00048, A=0.823 \pm 0.013, \bar{\rho}=0.141 \pm 0.019, \bar{\eta}=0.349 \pm 0.012$ 21.

Comparing the CKM elements resulting from Eq. (7.6) and Eq. (7.7) gives us the Table 7.1. We see that a relevant relation between $\lambda_{u}, \lambda_{d}$ and $\lambda$ is

$$
\lambda=\lambda_{d}-\lambda_{u}
$$

Moreover by looking at the $V_{13}$ and $V_{31}$ terms from Table (7.1) it is reasonable to make an ansätz in which

$$
\lambda_{u}=\frac{m_{u}}{m_{t}} \quad \text { and } \quad \lambda_{d} \approx \lambda
$$

Before using the above ansätz to construct quark mass matrices $\mathcal{M}_{u}$ and $\mathcal{M}_{d}$ let us discuss on which kind of matrix forms will be the candidate to describe the quark masses and mixings. 
Table 7.1: Wolfenstein vs. Wolfenstein-like parameters for CKM matrix

\begin{tabular}{|c|c|c|}
\hline CKM elements & Wolfenstein parameters & Wolfenstein-like parameter \\
\hline \hline$V_{11}$ & $1-\frac{\lambda^{2}}{2}$ & $1-\left(\frac{\lambda_{u}^{2}}{2}+\frac{\lambda_{d}^{2}}{2}-\lambda_{d} \lambda_{u}\right)$ \\
\hline$V_{12}$ & $\lambda$ & $\lambda_{d}-\lambda_{u}$ \\
\hline $\operatorname{Re}\left(V_{13}\right)$ & $A \lambda^{3} \rho$ & $A_{u} \lambda_{u}^{3}\left(1-\rho_{u}\right)+A_{d} \lambda_{d}^{3}\left(\rho_{d}-\lambda_{u} / \lambda_{d}\right)$ \\
$I m\left(V_{13}\right)$ & $-A \lambda^{3} \eta$ & $A_{u} \lambda_{u}^{3} \eta_{u}-A_{d} \lambda_{d}^{3} \eta_{d}$ \\
\hline$V_{21}$ & $-\lambda$ & $-\left(\lambda_{d}-\lambda_{u}\right)$ \\
\hline$V_{22}$ & $1-\frac{\lambda^{2}}{2}$ & $1-\left(\frac{\lambda_{u}^{2}}{2}+\frac{\lambda_{d}^{2}}{2}-\lambda_{d} \lambda_{u}\right)$ \\
\hline$V_{23}$ & $A \lambda^{2}$ & $A_{d} \lambda_{d}^{2}-A_{u} \lambda_{u}^{2}$ \\
\hline $\operatorname{Re}\left(V_{31}\right)$ & $A \lambda^{3}(1-\rho)$ & $A_{d} \lambda_{d}^{3}\left(1-\rho_{d}\right)+A_{u} \lambda_{u}^{3}\left(\rho_{u}-\lambda_{d} / \lambda_{u}\right)$ \\
$I m\left(V_{31}\right)$ & $-A \lambda^{3} \eta$ & $A_{u} \lambda_{u}^{3} \eta_{u}-A_{d} \lambda_{d}^{3} \eta_{d}$ \\
\hline$V_{32}$ & $-A \lambda^{2}$ & $-\left(A_{d} \lambda_{d}^{2}-A_{u} \lambda_{u}^{2}\right)$ \\
\hline$V_{33}$ & 1 & 1 \\
\hline
\end{tabular}

\subsection{A general quark mass matrix and its eigenvalues}

There has been several attempts unraveling the quark mass problem and the literature. Especially, there are studies of the mass matrix presuming that it is hermitian, symmetric [126], [127], [128], [129] or anti-hermitian [130]. These works have had the same goal which is to reduce the number of parameters in the quark mass matrices $\mathcal{M}_{u, d}$ (18 for each if they are complex) by putting zeros in the matrix elements, called zero texture of quark mass matrices 126, 131, 132. This motivated us to introduce a suitable matrix texture in order to acquire quark masses after diagonalizing the matrix.

We first start with a general quark mass matrix of three generations having a form of

$$
\mathcal{M}_{q}=\left(\begin{array}{ccc}
a & b & c \\
m & e & f \\
n & r & h
\end{array}\right)
$$

where all elements are complex in this case. It is worth noticing that one must be really careful when inputting zeros in the matrix since this action may lead to at least one zero eigenvalue. 
Therefore our work takes one more step further to look into eigenvalues of the matrix 7.10 before making any assumption. Eventually we expect the eigenvalues $\lambda_{i}$ of the matrix to be the quark masses, say $m_{d}, m_{s}, m_{b}$ for the down-quark sector. Eigenvalues of the matrix 7.10 are expressed as

$$
\begin{aligned}
\lambda_{1}= & \frac{1}{3 \sqrt[3]{2}} \cdot A \\
& -\frac{\sqrt[3]{2}}{3} \cdot \frac{-a^{2}+a h+e a-3 b m-3 c n-3 f r-h^{2}+e h-e^{2}}{A} \\
& +\frac{1}{3}(a+h+e) \\
\lambda_{2}= & -\frac{1}{6 \sqrt[3]{2}}(1-i \sqrt{3}) \cdot A \\
& +\frac{1}{3 \cdot 2^{2 / 3}}(1+i \sqrt{3}) \cdot \frac{-a^{2}+a h+e a-3 b m-3 c n-3 f r-h^{2}+e h-e^{2}}{A} \\
& +\frac{1}{3}(a+h+e) \\
\lambda_{3}= & \frac{1}{6 \sqrt[3]{2}}(1+i \sqrt{3}) \cdot A \\
& +\frac{1}{3 \cdot 2^{2 / 3}}(1-i \sqrt{3}) \cdot \frac{-a^{2}+a h+e a-3 b m-3 c n-3 f r-h^{2}+e h-e^{2}}{A} \\
& +\frac{1}{3}(a+h+e)
\end{aligned}
$$

where

$$
\begin{aligned}
A=\quad & 2 a^{3}-3 a^{2} h-3 e a^{2} \\
+ & \sqrt{ }\left[4\left(-a^{2}+a h+e a-3 b m-3 c n-3 f r-h^{2}+e h-e^{2}\right)^{3}\right. \\
+ & \left(2 a^{3}-3 a^{2} h-3 e a^{2}+9 a b m+9 a c n-18 a f r-3 a h^{2}+12 e a h-3 e^{2} a+27 b f n-18 b h m\right. \\
& \left.\left.+9 e b m+9 c h n+27 c m r-18 e c n+9 f h r+9 e f r+2 h^{3}-3 e h^{2}-3 e^{2} h+2 e^{3}\right)^{2}\right] \\
+ & 9 a b m+9 a c n-18 a f r-3 a h^{2}+12 e a h-3 e^{2} a+27 b f n-18 b h m+9 e b m \\
+ & \left.9 c h n+27 c m r-18 e c n+9 f h r+9 e f r+2 h^{3}-3 e h^{2}-3 e^{2} h+2 e^{3}\right\}^{1 / 3}
\end{aligned}
$$

By analyzing the above eigenvalues if one makes any assumption then it is necessary to keep 3 parameters $a, e, h$. Note that $|a|<|e| \ll|h|$. Furthermore $A$ is proven to be complex for any $a, e, h$ 
in this case. We also have $\lambda_{1} \gg \lambda_{2}>\lambda_{3}$ so a correspondence can be established

$$
\lambda_{1} \longleftrightarrow m_{b}, \lambda_{2} \longleftrightarrow m_{s}, \lambda_{3} \longleftrightarrow m_{d}
$$

\subsection{Finding quark mass matrices $\mathcal{M}_{u}$ and $\mathcal{M}_{d}$}

Acknowledging the facts and arguments in previous sections, we are now on the mission of finding possible forms of quark mass matrices. Recall that we have set $\lambda_{u}=\frac{m_{u}}{m_{t}}$ and $\lambda_{d} \approx \lambda$.

\subsubsection{Down-quark sector}

The current data from 21] record that $m_{d}=0.0048 \mathrm{GeV}, m_{s}=0.095 \mathrm{GeV}$ and $m_{b}=$ $4.42 \mathrm{GeV}$. A suggested form of $M_{d}$ is

$$
\mathcal{M}_{d}=\left(\begin{array}{ccc}
a_{d} & b_{d} & c_{d} \\
b_{d}^{*} & e_{d} & f_{d} \\
c_{d}^{*} & f_{d}^{*} & h_{d}
\end{array}\right) \rightarrow \mathcal{M}_{d}^{\dagger}=\left(\begin{array}{ccc}
a_{d}^{*} & b_{d} & c_{d} \\
b_{d}^{*} & e_{d}^{*} & f_{d} \\
c_{d}^{*} & f_{d}^{*} & h_{d}^{*}
\end{array}\right)
$$

Therefore,

$$
M_{d}=\mathcal{M}_{d} \mathcal{M}_{d}^{\dagger}=\left(\begin{array}{ccc}
a_{d} & b_{d} & c_{d} \\
b_{d}^{*} & e_{d} & f_{d} \\
c_{d}^{*} & f_{d}^{*} & h_{d}
\end{array}\right)\left(\begin{array}{ccc}
a_{d}^{*} & b_{d} & c_{d} \\
b_{d}^{*} & e_{d}^{*} & f_{d} \\
c_{d}^{*} & f_{d}^{*} & h_{d}^{*}
\end{array}\right)
$$

We shall compare 7.17 with the product $\mathcal{M}_{d} \mathcal{M}_{d}^{\dagger}$ implied from the neutrino mass model $[92$ (up to $\mathcal{O}\left(\lambda_{d}^{3}\right)$ since $\left.\lambda_{d} \approx \lambda\right)$

$$
\mathcal{M}_{d} \mathcal{M}_{d}^{\dagger}=\left(\begin{array}{ccc}
\left(1-\lambda_{d}^{2}\right) m_{d}^{2}+\lambda_{d}^{2} m_{s}^{2} & \lambda_{d}\left(m_{s}{ }^{2}-m_{d}{ }^{2}\right) & A_{d} \lambda_{d}^{3} m_{b}^{2}\left(\rho_{d}-i \eta_{d}\right) \\
\lambda_{d}\left(m_{s}{ }^{2}-m_{d}{ }^{2}\right) & \lambda_{d}^{2} m_{d}^{2}+\left(1-\lambda_{d}^{2}\right) m_{s}{ }^{2} & A_{d} \lambda_{d}^{2}\left(m_{b}{ }^{2}-m_{s}{ }^{2}\right) \\
A_{d} \lambda_{d}^{3} m_{b}^{2}\left(\rho_{d}+i \eta_{d}\right) & A_{d} \lambda_{d}^{2}\left(m_{b}{ }^{2}-m_{s}{ }^{2}\right) & m_{b}{ }^{2}
\end{array}\right)
$$


This results in the following equations

$$
\begin{aligned}
& M_{d}^{11}=\left|a_{d}\right|^{2}+\left|b_{d}\right|^{2}+\left|c_{d}\right|^{2}=\left(1-\lambda_{d}^{2}\right) m_{d}^{2}+\lambda_{d}^{2} m_{s}^{2} \\
& M_{d}^{22}=\left|b_{d}\right|^{2}+\left|e_{d}\right|^{2}+\left|f_{d}\right|^{2}=\left(1-\lambda_{d}^{2}\right) m_{s}^{2}+\lambda_{d}^{2} m_{d}^{2} \\
& M_{d}^{33}=\left|c_{d}\right|^{2}+\left|f_{d}\right|^{2}+\left|h_{d}\right|^{2}=m_{b}^{2} \\
& M_{d}^{12}=a_{d} b_{d}+b_{d} e_{d}^{*}+c_{d} f_{d}^{*}=\lambda_{d}\left(m_{s}^{2}-m_{d}^{2}\right) \\
& M_{d}^{21}=M_{d}^{12^{*}} \\
& M_{d}^{13}=a_{d} c_{d}+b_{d} f_{d}+c_{d} h_{d}^{*}=A_{d} \lambda_{d}^{3} m_{b}^{2}\left(\rho_{d}-i \eta_{d}\right) \\
& M_{d}^{31}=M_{d}^{13^{*}} \\
& M_{d}^{23}=b_{d}^{*} c_{d}+e_{d} f_{d}+f_{d} h_{d}^{*}=A_{d} \lambda_{d}^{2}\left(m_{b}^{2}-m_{s}^{2}\right) \\
& M_{d}^{32}=M_{d}^{23 *}
\end{aligned}
$$

Since only $a_{d}, e_{d}, h_{d}$ must stay in the matrix it is reasonable to assume that $\left|b_{d}\right| \ll 1$ and therefore we can replace $b_{d}$ and $b_{d}^{*}$ by zeros. Moreover let us take $a_{d}, e_{d}$ to be real as we would like to reduce number of entries. After these assumptions the remaining parameters are exhibited in the following equations

$$
\begin{aligned}
\left|a_{d}\right|^{2}+\left|c_{d}\right|^{2} & =\left(1-\lambda_{d}^{2}\right) m_{d}^{2}+\lambda_{d}^{2} m_{s}^{2} \\
\left|e_{d}\right|^{2}+\left|f_{d}\right|^{2} & =\left(1-\lambda_{d}^{2}\right) m_{s}^{2}+\lambda_{d}^{2} m_{d}^{2} \\
\left|c_{d}\right|^{2}+\left|f_{d}\right|^{2}+\left|h_{d}\right|^{2} & =m_{b}^{2} \\
c_{d} f_{d}^{*} & =\lambda_{d}\left(m_{s}^{2}-m_{d}^{2}\right) \\
a_{d} c_{d}+c_{d} h_{d}^{*} & =A_{d} \lambda_{d}^{3} m_{b}^{2}\left(\rho_{d}-i \eta_{d}\right) \\
e_{d} f_{d}+f_{d} h_{d}^{*} & =A_{d} \lambda_{d}^{2}\left(m_{b}^{2}-m_{s}^{2}\right)
\end{aligned}
$$

If setting

$$
\begin{aligned}
& a_{d}=a_{d 1}, e_{d}=e_{d 1}, \\
& c_{d}=c_{d 1}+i c_{d 2}, f_{d}=f_{d 1}+i f_{d 2}, h_{d}=h_{d 1}+i h_{d 2},
\end{aligned}
$$


we have

$$
\begin{aligned}
a_{d 1}^{2}+c_{d 1}^{2}+c_{d 2}^{2} & =\left(1-\lambda_{d}^{2}\right) m_{d}^{2}+\lambda_{d}^{2} m_{s}^{2} \\
e_{d 1}^{2}+f_{d 1}^{2}+f_{d 2}^{2} & =\left(1-\lambda_{d}^{2}\right) m_{s}^{2}+\lambda_{d}^{2} m_{d}^{2} \\
c_{d 1}^{2}+c_{d 2}^{2}+f_{d 1}^{2}+f_{d 2}^{2}+h_{d 1}^{2}+h_{d 2}^{2} & =m_{b}^{2} \\
c_{d 1} f_{d 1}+c_{d 2} f_{d 2} & =\lambda_{d}\left(m_{s}^{2}-m_{d}^{2}\right) \\
a_{d 1} c_{d 1}+c_{d 1} h_{d 1}+c_{d 2} h_{d 2} & =A_{d} \lambda_{d}^{3} \rho_{d} m_{b}^{2} \\
a_{d 1} c_{d 2}+c_{d 2} h_{d 1}-c_{d 1} h_{d 2} & =-A_{d} \lambda_{d}^{3} \eta_{d} m_{b}^{2} \\
e_{d 1} f_{d 1}+f_{d 1} h_{d 1}+f_{d 2} h_{d 2} & =A_{d} \lambda_{d}^{2}\left(m_{b}^{2}-m_{s}^{2}\right)
\end{aligned}
$$

Notice that there are 8 unknowns and 7 equations. Therefore it is necessary to have other assumptions on parameters of the matrix. Because of the hierarchy $m_{d} \ll m_{s} \ll m_{b}$ one can predict that

$$
\begin{aligned}
\left|a_{d}\right| & \sim \mathcal{O}\left(m_{d}\right), \\
\left|e_{d}\right| & \sim \mathcal{O}\left(m_{s}\right), \\
\left|h_{d}\right| & \sim \mathcal{O}\left(m_{b}\right) .
\end{aligned}
$$

Let us take

$$
h_{d 1}=0.999 m_{b} \quad \text { and } \quad h_{d 2} \approx 0
$$

and define

$$
\begin{aligned}
a_{d 1} & =b m_{d}, \\
e_{d 1} & =c m_{s}, \\
c_{d 1} & =d m_{s}, \quad c_{d 2}=k m_{s}, \\
f_{d 1} & =p m_{s} .
\end{aligned}
$$

If $b=\sqrt{r\left(1-\lambda_{d}^{2}\right)}$ where $r$ is some proportional factor and will be constraint later then Eq. 7.36 gives

$$
d=\frac{A_{d} \lambda_{d}^{3} \rho_{d} m_{b}^{2}}{\left(b m_{d}+h_{d 1}\right) m_{s}}
$$


On the other hand from Eq. 7.32 we have

$$
\begin{aligned}
& b^{2} m_{d}^{2}+d^{2} m_{s}^{2}+k^{2} m_{s}^{2}=\left(1-\lambda_{d}^{2}\right) m_{d}^{2}+\lambda_{d}^{2} m_{s}^{2} \\
\Rightarrow & k=\sqrt{\frac{\left(1-\lambda_{d}^{2}-b^{2}\right) m_{d}^{2}+\lambda_{d}^{2} m_{s}^{2}}{m_{s}^{2}}-d^{2}}
\end{aligned}
$$

The fact that the determinant of the $\mathcal{M}_{d}$, denoted $\operatorname{Det}\left(\mathcal{M}_{d}\right)$ must satisfy

$$
\operatorname{Det}\left(\mathcal{M}_{d}\right) \propto m_{d} m_{s} m_{b}
$$

so that one can obtain 3 eigenvalues corresponding to 3 masses $m_{d}, m_{s}, m_{b}$. Since $\operatorname{Det}\left(\mathcal{M}_{d}\right) \approx$ $a_{d 1} e_{d 1} h_{d 1}$ it is reasonable to assume that

$$
a_{d 1} e_{d 1} \approx m_{d} m_{s} \quad \text { as } \quad h_{d 1} \approx m_{b}
$$

From this we have

$$
c \approx 1 / b
$$

Hence Eq. 7.38 in which

$$
c^{2} m_{s}^{2}+p^{2} m_{s}^{2}+f_{d 2}^{2}=\left(1-\lambda_{d}^{2}\right) m_{s}^{2}+\lambda_{d}^{2} m_{d}^{2},
$$

results in

$$
\begin{aligned}
f_{d 2} & =\lambda_{d} m_{d} \\
p & =\sqrt{\left(1-\lambda_{d}^{2}\right)-c^{2}} .
\end{aligned}
$$

Since it is required that factors $b, c, d, k, p$ are real we can constrain the values for $A_{d}$ and $\rho_{d}$. Numerically we found

$$
r \geq 1.11 \quad, \quad A_{d} \leq 0.7 \quad \text { and } \quad \rho_{d} \leq 0.6 \text {. }
$$

Therefore we obtain

$$
M_{d}=\left(\begin{array}{ccc}
b m_{d} & 0 & d m_{s}+i\left(k m_{s}\right) \\
0 & m_{s} / b & p m_{s}+i\left(\lambda_{d} m_{d}\right) \\
d m_{s}-i\left(k m_{s}\right) & p m_{s}-i\left(\lambda_{d} m_{d}\right) & 0.999 m_{b}
\end{array}\right) .
$$

From this junction some comments are in order 
- Wolfenstein-like parameters from our model with the appropriate assumptions having such a good flexibility allow us to vary their values in order to get a reasonable agreement with experimental data.

- It is vital to also have off-diagonal terms in the quark mass matrix since they account for the mixings between quark generations.

As an example, let us take $r=1.11, A_{d}=0.7$, and $\rho_{d}=0.6$ we have a numerical form of $\mathcal{M}_{d}$

$$
\mathcal{M}_{d}=\left(\begin{array}{ccc}
1.0266 m_{d} & 0 & 0.2224 m_{s}+i\left(0.0302 m_{s}\right) \\
0 & 0.9741 m_{s} & 0.0208 m_{s}+i\left(\lambda_{d} m_{d}\right) \\
0.2224 m_{s}-i\left(0.0302 m_{s}\right) & 0.0208 m_{s}-i\left(\lambda_{d} m_{d}\right) & 0.999 m_{b}
\end{array}\right) .
$$

where $\lambda_{d}=\lambda=0.225$. The eigenvalues of this matrix are listed (in $G e V$ )

$$
\begin{aligned}
& \lambda_{1}=4.41966, \\
& \lambda_{2}=0.09254, \\
& \lambda_{3}=0.00482 .
\end{aligned}
$$

It is noticed that the combination of $r=1.11, A_{d}=0.7$, and $\rho_{d}=0.6$ gives the eigenvalues that are closest to the absolute masses given in [21] which are

$$
m_{d}=0.0048 \mathrm{GeV}, m_{s}=0.095 \mathrm{GeV}, m_{b}=4.42 \mathrm{GeV} .
$$

Finally, plugging the above values into Eq. 7.37) we obtain $\left|\eta_{d}\right| \simeq 0.1$.

\subsubsection{Up-quark sector}

Masses of up-type quarks are given by 21 as

$$
m_{u}=0.0023 \mathrm{GeV}, \quad m_{c}=1.275 \mathrm{GeV}, \quad m_{t}=173 \mathrm{GeV} .
$$


The suggested form of $\mathcal{M}_{u}$ is as follows

$$
\mathcal{M}_{u}=\left(\begin{array}{ccc}
a_{u} & b_{u} & c_{u} \\
b_{u}^{*} & e_{u} & f_{u} \\
c_{u}^{*} & f_{u}^{*} & h_{u}
\end{array}\right) \rightarrow \mathcal{M}_{u}^{\dagger}=\left(\begin{array}{ccc}
a_{u}^{*} & b_{u} & c_{u} \\
b_{u}^{*} & e_{u}^{*} & f_{u} \\
c_{u}^{*} & f_{u}^{*} & h_{u}^{*}
\end{array}\right)
$$

Therefore,

$$
M_{u}=\mathcal{M}_{u} \mathcal{M}_{u}^{\dagger}=\left(\begin{array}{ccc}
a_{u} & b_{u} & c_{u} \\
b_{u}^{*} & e_{u} & f_{u} \\
c_{u}^{*} & f_{u}^{*} & h_{u}
\end{array}\right)\left(\begin{array}{ccc}
a_{u}^{*} & b_{u} & c_{u} \\
b_{u}^{*} & e_{u}^{*} & f_{u} \\
c_{u}^{*} & f_{u}^{*} & h_{u}^{*}
\end{array}\right)
$$

If one compare 7.64 with the product $\mathcal{M}_{u} \mathcal{M}_{u}^{\dagger}$ implied from a neutrino mass model 92 (up to $\mathcal{O}\left(\lambda_{u}^{2}\right)$ since $\left.\lambda_{u}=m_{u} / m_{t}\right)$ :

$$
\mathcal{M}_{u} \mathcal{M}_{u}^{\dagger}=\left(\begin{array}{ccc}
\left(1-\lambda_{u}^{2}\right) m_{u}{ }^{2}+\lambda_{u}^{2} m_{c}{ }^{2} & \lambda_{u}\left(m_{c}{ }^{2}-m_{u}{ }^{2}\right) & 0 \\
\lambda_{u}\left(m_{c}{ }^{2}-m_{u}{ }^{2}\right) & \lambda_{u}^{2} m_{u}{ }^{2}+\left(1-\lambda_{u}^{2}\right) m_{c}{ }^{2} & A_{u} \lambda_{u}^{2}\left(m_{t}{ }^{2}-m_{c}{ }^{2}\right) \\
0 & A_{u} \lambda_{u}^{2}\left(m_{t}{ }^{2}-m_{c}{ }^{2}\right) & m_{t}{ }^{2}
\end{array}\right)
$$

the following equations come out as a result

$$
\begin{aligned}
& M_{u}^{11}=\left|a_{u}\right|^{2}+\left|b_{u}\right|^{2}+\left|c_{u}\right|^{2}=\left(1-\lambda_{u}^{2}\right) m_{u}{ }^{2}+\lambda_{u}^{2} m_{c}{ }^{2} \\
& M_{u}^{22}=\left|b_{u}\right|^{2}+\left|e_{u}\right|^{2}+\left|f_{u}\right|^{2}=\left(1-\lambda_{u}^{2}\right) m_{c}{ }^{2}+\lambda_{u}^{2} m_{u}{ }^{2} \\
& M_{u}^{33}=\left|c_{u}\right|^{2}+\left|f_{u}\right|^{2}+\left|h_{u}\right|^{2}=m_{t}^{2} \\
& M_{u}^{12}=a_{u} b_{u}+b_{u} e_{u}^{*}+c_{u} f_{u}^{*}=\lambda_{u}\left(m_{c}^{2}-m_{u}^{2}\right) \\
& M_{u}^{21}=M_{u}^{12^{*}} \\
& M_{u}^{13}=a_{u} c_{u}+b_{u} f_{u}+c_{u} h_{u}^{*}=0 \\
& M_{u}^{31}=M_{u}^{13^{*}} \\
& M_{u}^{23}=b_{u}^{*} c_{u}+e_{u} f_{u}+f_{u} h_{u}^{*}=A_{u} \lambda_{u}^{2}\left(m_{t}^{2}-m_{c}^{2}\right) \\
& M_{u}^{32}=M_{u}^{23^{*}}
\end{aligned}
$$

A similar analysis to that of the down quark sector can be carried out here but note that the elements $M_{u}^{13}$ and $M_{u}^{31}$ are no longer non-zero as opposed to the case of down quark sector. Again 
we make such assumptions that

$$
\left|b_{u}\right| \ll 1 \text { and } a_{u}, e_{u} \text { is real } \text {. }
$$

and set

$$
\begin{aligned}
& a_{u}=a_{u 1}, e_{u}=e_{u 1}, \\
& c_{u}=c_{u 1}+i c_{u 2}, f_{u}=f_{u 1}+i f_{u 2}, h_{u}=h_{u 1}+i h_{u 2},
\end{aligned}
$$

Using the above set-ups we have

$$
\begin{aligned}
a_{u 1}^{2}+c_{u 1}^{2}+c_{u 2}^{2} & =\left(1-\lambda_{u}^{2}\right) m_{u}^{2}+\lambda_{u}^{2} m_{c}^{2} \\
e_{u 1}^{2}+f_{u 1}^{2}+f_{u 2}^{2} & =\left(1-\lambda_{u}^{2}\right) m_{c}^{2}+\lambda_{u}^{2} m_{u}^{2} \\
c_{u 1}^{2}+c_{u 2}^{2}+f_{u 1}^{2}+f_{u 2}^{2}+h_{u 1}^{2}+h_{u 2}^{2} & =m_{t}^{2} \\
c_{u 1} f_{u 1}+c_{u 2} f_{u 2} & =\lambda_{u}\left(m_{c}^{2}-m_{u}^{2}\right) \\
a_{u 1} c_{u 1}+c_{u 1} h_{u 1}+c_{u 2} h_{u 2} & =0 \\
e_{u 1} f_{u 1}+f_{u 1} h_{u 1}+f_{u 2} h_{u 2} & =A_{u} \lambda_{u}^{2}\left(m_{t}^{2}-m_{c}^{2}\right)
\end{aligned}
$$

Unlike the down quark case, we now have only 6 equations to accommodate for 8 unknowns. Because of the hierarchy $m_{u} \ll m_{c} \ll m_{t}$ one can predict that

$$
\begin{aligned}
\left|a_{u}\right| & \sim \mathcal{O}\left(m_{u}\right), \\
\left|e_{u}\right| & \sim \mathcal{O}\left(m_{c}\right), \\
\left|h_{u}\right| & \sim \mathcal{O}\left(m_{t}\right) .
\end{aligned}
$$

Let us take

$$
h_{u 1}=0.999 m_{t} \quad \text { and } \quad h_{u 2} \approx 0 .
$$


and define

$$
\begin{aligned}
& a_{u 1}=b^{\prime} m_{u}, \\
& e_{u 1}=c^{\prime} m_{c}, \\
& f_{u 1}=p^{\prime} m_{c}, f_{u 2}=q^{\prime} m_{c}, \\
& c_{u 1}=d^{\prime} m_{u} .
\end{aligned}
$$

If $c^{\prime}=\sqrt{r^{\prime}\left(1-\lambda_{u}^{2}\right)}$ then from Eq. 7.79 we obtain

$$
p^{\prime}=\frac{A_{u} \lambda_{u}^{2}\left(m_{t}^{2}-m_{c}^{2}\right)}{\left(c^{\prime} m_{c}+h_{u 1}\right) m_{c}}
$$

Once we have $c^{\prime}$ and $p^{\prime}$, Eq. 7.75 gives

$$
\begin{aligned}
& c^{\prime 2}+{p^{\prime}}^{2}+q^{\prime 2}=\frac{\left(1-\lambda_{u}^{2}\right) m_{c}^{2}+\lambda_{u}^{2} m_{u}^{2}}{m_{c}^{2}} \\
\Rightarrow & q^{\prime}=\sqrt{\frac{\left(1-\lambda_{u}^{2}\right) m_{c}^{2}+\lambda_{u}^{2} m_{u}^{2}}{m_{c}^{2}}-{c^{\prime}}^{2}-{p^{\prime}}^{2}}
\end{aligned}
$$

The fact that the determinant of the $\mathcal{M}_{u}$, denoted $\operatorname{Det}\left(\mathcal{M}_{u}\right)$ must satisfy

$$
\operatorname{Det}\left(\mathcal{M}_{u}\right) \propto m_{u} m_{c} m_{t}
$$

so that one can obtain 3 eigenvalues corresponding to 3 masses $m_{u}, m_{c}, m_{t}$. Since $\operatorname{Det}\left(\mathcal{M}_{u}\right) \approx$ $a_{u 1} e_{u 1} h_{u 1}$ it is reasonable to assume that

$$
a_{u 1} e_{u 1} \approx m_{u} m_{c} \text { since } h_{u 1} \approx m_{t} .
$$

From this we have

$$
b^{\prime} \approx 1 / c^{\prime} .
$$

Next, Eq. 7.74

$$
d^{\prime 2} m_{u}^{2}+c_{u 2}^{2}=\left(1-\lambda_{u}^{2}-{b^{\prime}}^{2}\right) m_{u}^{2}+\lambda_{u}^{2} m_{c}^{2}
$$

results in

$$
\begin{aligned}
d^{\prime} & =\sqrt{\left(1-\lambda_{u}^{2}\right)-b^{\prime 2}} m_{u}, \\
c_{u 2} & =\lambda_{u} m_{c}
\end{aligned}
$$


Since it is required that factors $b^{\prime}, c^{\prime}, d^{\prime}, p^{\prime}, q^{\prime}$ are real we can constrain the values for $A_{u}$. Numerically we found

$$
r^{\prime}<1 \quad, \quad A_{u} \sim \mathcal{O}(1)
$$

Therefore we obtain

$$
\mathcal{M}_{u}=\left(\begin{array}{ccc}
b^{\prime} m_{u} & 0 & d^{\prime} m_{u}+i\left(\lambda_{u} m_{c}\right) \\
0 & c^{\prime} m_{c} & p^{\prime} m_{c}+i\left(q^{\prime} m_{c}\right) \\
d^{\prime} m_{u}-i\left(\lambda_{u} m_{c}\right) & p^{\prime} m_{c}-i\left(q^{\prime} m_{c}\right) & 0.999 m_{t}
\end{array}\right) .
$$

where $\lambda_{u}=m_{u} / m_{t}$. As an example, take $r^{\prime}=0.9$ and $A_{d}=0.9$ we have the numerical from of $\mathcal{M}_{u}$ as follows

$$
\mathcal{M}_{u} \simeq\left(\begin{array}{ccc}
1.05409 m_{u} & 0 & i\left(\lambda_{u} m_{c}\right) \\
0 & 0.94868 m_{c} & i\left(0.31623 m_{c}\right) \\
-i\left(\lambda_{u} m_{c}\right) & -i\left(0.31623 m_{c}\right) & 0.999 m_{t}
\end{array}\right)
$$

The eigenvalues of this matrix are listed (in $\mathrm{GeV}$ )

$$
\begin{aligned}
& \lambda_{1}=172.984, \\
& \lambda_{2}=1.20862, \\
& \lambda_{3}=0.00242 .
\end{aligned}
$$

Recall that data from 21] are given

$$
m_{u}=0.0023 \mathrm{GeV}, m_{c}=1.275 \mathrm{GeV}, m_{t}=173 \mathrm{GeV} .
$$

Up to this point, a few comments are included.

- The up-quark mass matrix receives more freedom in its parameter space as compared with the down-quark mass matrix.

- It also contains the off-diagonal terms to describe the mixings even though these terms are small in general. 
In conclusion, we have the following forms of down and up quark mass matrices.

$$
\begin{aligned}
& \mathcal{M}_{d}=\left(\begin{array}{ccc}
b m_{d} & 0 & d m_{s}+i\left(k m_{s}\right) \\
0 & m_{s} / b & p m_{s}+i\left(\lambda_{d} m_{d}\right) \\
d m_{s}-i\left(k m_{s}\right) & p m_{s}-i\left(\lambda_{d} m_{d}\right) & 0.999 m_{b}
\end{array}\right), \\
& \mathcal{M}_{u}=\left(\begin{array}{ccc}
b^{\prime} m_{u} & 0 & d^{\prime} m_{u}+i\left(\lambda_{u} m_{c}\right) \\
0 & c^{\prime} m_{c} & p^{\prime} m_{c}+i\left(q^{\prime} m_{c}\right) \\
d^{\prime} m_{u}-i\left(\lambda_{u} m_{c}\right) & p^{\prime} m_{c}-i\left(q^{\prime} m_{c}\right) & 0.999 m_{t}
\end{array}\right) .
\end{aligned}
$$

\subsection{Agreement with the experimental CKM matrix}

It is well-established that the experimental values of Wolfenstein parameters used to construct the CKM matrix are 21]

$$
\begin{array}{ll}
\lambda=0.22496 \pm 0.00048, & A=0.823 \pm 0.013, \\
\bar{\rho}=0.141 \pm 0.019, & \bar{\eta}=0.349 \pm 0.012 .
\end{array}
$$

Therefore we have the numerical form

$$
V_{C K M}=\left(\begin{array}{ccc}
0.974696 & 0.22496 & 0.0013-0.00327 i \\
-0.22496 & 0.974696 & 0.04165 \\
0.00805-0.00327 i & -0.04165 & 1
\end{array}\right) .
$$

On the other hand the model of neutrino masses and mixings has constrained

$$
\begin{aligned}
& \lambda_{d}=\lambda, \quad A_{d} \leq 0.7, \quad \rho_{d} \leq 0.6, \quad \eta_{d} \simeq 0.1 ; \\
& \lambda_{u}=m_{u} / m_{t}, \quad A_{u}=0.9, \quad \rho_{u}=\eta_{u}=0 .
\end{aligned}
$$

It is worth emphasizing that the parameters in our model are quite flexible so one can play with values of parameters to get the best fit with the experimental $V_{C K M}$. An example of the numerical 
$V_{C K M}$ resulting from the model is

$$
\begin{aligned}
V_{C K M-\text { model }}= & U_{u L}^{\dagger} U_{d L} \\
& =\left(\begin{array}{ccc}
0.974699 & -0.224947 & 0.001195-0.000797 i \\
-0.224947 & 0.974699 & 0.035425 \\
0.006774-0.000797 i & -0.035425 & 1
\end{array}\right)
\end{aligned}
$$

Comparing Eq. (7.106) and 7.109) we see that our result is in a good agreement with the experimental CKM matrix. This may help increase the reliability of the quark mass matrices that we have found.

Nevertheless, it is not the end of the story. Even though we are able to construct the mass matrices from well-established experimental data, this work has not been completed without understanding what kinds of dynamical mechanisms are behind the results. To us, this step is very important because it may lead to a possibility of unravelling the mystery of the origin of masses and mixings. This work is now in progress and will be reported in our upcoming paper 133. 


\section{Chapter 8}

\section{Conclusion}

In this thesis we have presented the model of neutrino masses and mixings and discussed in details some of its phenomenological implications related to lepton and quark mixing matrices, lepton flavor violation in rare decays such as $\mu \rightarrow e \gamma$ and $\mu-e$ conversion, and the possible construction of quark mass matrices. These works have contributed to the overall understanding of physics within and beyond the standard model in the sense that they have not only helped explain experimental data but also predicted and suggested search regions for near future experiments.

We have started with the review of the Standard Model of particle physics in Chapter 2 and extensions to SM due to the discovery of neutrino oscillation indicating neutrinos have nonzero masses in Chapter 3 . The urge for right-handed neutrinos to be detected at the LHC has motivated the EW- $\nu_{R}$ model presented in Chapter 4. In this model $\nu_{R}$ 's are non-sterile and can acquire their Majorana masses at the electroweak scale $\Lambda_{E W} \approx 246 \mathrm{GeV}$. In order to not affect

the $Z$-width decay data, $M_{R}$ must be bounded from below at $M_{Z} / 2$. The EW-scale $M_{R}$ can be naturally achieved by adding the mirror fermions (leptons and quarks) and 2 scalar triplets $\tilde{\chi}$ and $\xi$ to the SM. Note that $\mathrm{EW}-\nu_{R}$ model also preserve the SM gauge group.

The fact is that any model, if introduced, has to be considered within the experimental constraints. A good agreement with experimental data results in increasing model's reliability. The EW- $\nu_{R}$ model has successfully passed the test of the electroweak precision on the oblique parameters 
$\tilde{S}, \tilde{T}$ and constraints from 125-GeV SM-like scalar. Since EW- $\nu_{R}$ adds extra chiral fermions (mirror leptons and quarks) that contribute positively to the $\tilde{S}$ parameter the first check of experimental constraints on $\tilde{S}$ and $\tilde{T}$ is very important. It is shown in Chapter 4 that the agreement has come from the evidence in which the large positive contribution to $\tilde{S}$ from the mirror fermions are almost cancelled by the negative contributions from the scalar triplets. Fig. 4.1) would provide a better illustration to the point we have made here. The second check is on the accommodation for the 125-GeV Higgs boson. By adding a complex scalar doublet $\Phi_{2 M}$ to the minimal EW- $\nu_{R}$ model which only couples to the mirror fermions we have shown a scalar spectrum that contains at least one Higgs field with the desired properties as required by experiment.

The highlight of this thesis is the model of neutrino masses and mixings presented in Chapter 5. It is put forward by the big puzzle why the CKM matrix for quarks is too different from the PMNS matrix for leptons and the fact that $U_{P M N S} \simeq U_{C W}$ conjectured by Cabibbo and Wolfenstein independently. We have found that the discrete $A_{4}$ symmetry is one of the possibilities that can give rise to the $U_{C W}$. We then apply this symmetry to the minimal EW- $\nu_{R}$ model in order to achieve $U_{C W}$ in the neutrino sector instead of the charged lepton sector as in some standard scenarios described in Chapter 5. The driving forces are as follows

(1) We are able to avoid involving many Higgs doublets (5 or so) causing potential problems with the $125-\mathrm{GeV}$ scalar Higgs.

(2) The Dirac neutrino mass matrix entails a Higgs singlet that does not suffer any constraints from the LHC. Therefore we have more freedom in adding singlets into our model.

Indeed we do have an extension to 4 Higgs singlets in the model considering the need of having offdiagonal elements in the Dirac neutrino mass matrix. It has been shown that the PMNS matrix of the leptons consists of $U_{l L}$ from couplings to the Higgs doublet and $U_{\nu L}$ from couplings to the Higgs singlets whereas the CKM matrix of the quarks comes totally from couplings to the Higgs doublet. 
Hence one expects a natural difference between the PMNS and CKM matrices. Furthermore $A_{4}$ symmetry is also required to be slightly broken to avoid the case of degenerate charged leptons. From this we propose an anszät to have $U_{l L}$ expressed as the Wolfenstein-like unitary matrix. This leads to the construction of the lepton mass matrix "squared", namely $\mathcal{M}_{l} \mathcal{M}_{l}^{\dagger}$. Reasons to introduce $\mathcal{M}_{l} \mathcal{M}_{l}^{\dagger}$ are: 1) from this it is possible to construct the lepton mass matrix $\mathcal{M}_{l}$ with further assumptions; 2) since charged leptons and quarks acquire their masses by couplings to the Higgs doublet one can make an analogy to the lepton sector to find the quark mass matrix "squared" $\mathcal{M}_{q} \mathcal{M}_{q}^{\dagger}$ then deduce the quark mass matrix $\mathcal{M}_{q}$.

Two very interesting phenomenological implications of the model of neutrino masses and mixings are lepton flavor violating processes $\mu \rightarrow e \gamma$ and $\mu-e$ conversion discussed thoroughly in Chapter 6. They have been investigated via one-loop induced diagrams incorporating in 4 Higgs singlets in the loop. We also perform numerical analyses on the parameter space of these processes regarding the current limits and projected sensitivities and find out that the Yukawa couplings of Higgs singlets to the leptons is small $\sim \mathcal{O}\left(10^{-5}-10^{-3}\right)$. Such small couplings can be quite interesting because they affect the way we search for mirror particles and Majorana right-handed neutrinos. In this case these particles might decay outside the beam pipe and inside the silicon vertex detectors in the form of displaced vertices with decay lengths $>1 \mathrm{~mm}$ or so. The singlet and triplet scalars in our model escape as missing energies. Additionally, although there are many contributors to $\mu-e$ conversion rate such as photon $(Z)$ exchange, Higgs exchange with light or heavy quarks, and the box diagram, the photon exchange is proven to be dominant. Therefore we are able to find a relation between $\mu-e$ conversion and $\mu \rightarrow e \gamma$ within a good approximation. This relation is attractive because the current limit from $\mu \rightarrow e \gamma$ excludes almost half of the searched region for the branching ratio of $\mu-e$ conversion when we plot them on the same parameter space. The results are shown in Figs. 6.9 - 6.12). Therefore our work may help narrow down future searches for $\mu-e$ conversion at Fermilab/Mu2e, J-PARC/COMET and PRISM within this model. 
In addition to phenomenological implications of the lepton sector, the model of neutrino masses and mixings also leads us to implications on quark mass matrices. Chapter 7 is dedicated

to study these. As inspired in the consideration of the lepton mass matrix squared $\mathcal{M}_{l} \mathcal{M}_{l}^{\dagger}$ the quark mass one is proposed to have similar form with its own parameters. Due to the large number of parameters it is necessary to trim some of them down by making assumptions. Using the knowledge of quark masses and the CKM matrix we are able to constrain the remaining parameters. As a result we obtain the up and down quark mass matrices whose parameters can be used to reconstruct the CKM matrix. Overall, the numerical forms of up and down quark mass matrices as well as the CKM matrix within this model are in good agreement with the experimental data. We have recently begun a study of dynamical mechanisms behind the quark mass matrices that we have found. It is crucial to understand the origin of quark masses and the very distinctive mass hierarchies.

Last but not least, a model of neutrino masses and mixings involving mirror fermions, Majorana right-handed neutrinos and Higgs singlet fields could lead to several consequences.

\section{Which evidence support this model?}

The positive signals from lepton flavor violating rare decays $\mu \rightarrow e \gamma$ and $\mu-e$ conversion and the forms of lepton and quark mass matrices are the main pieces of evidence supporting this model.

\section{Why haven't we observed mirror fermions?}

We were showing in Eqs. 4.6) and (4.19) that the mirror fermions can couple to SM fermions through the interactions involving the Higgs singlet $\phi_{S}$. Since in EW- $\nu_{R}$ model, $\phi_{S}$ has mass of 
$\mathcal{O}\left(10^{5} \mathrm{eV}\right)$, mirror fermions can probably enter the following decay modes

$$
\begin{aligned}
& e_{R}^{M} \rightarrow e_{L}+\phi_{S}, \\
& q_{R}^{M} \rightarrow q_{L}+\phi_{S} .
\end{aligned}
$$

Hence mirror fermions are not stable and decay into the SM fermions. The mirror quarks could not form mirror-hadrons through their strong nuclear interactions because they had already decayed before those hadrons could be formed. The distinguished signals of mirror fermions at the LHC are like-sign dilepton events with missing energies + jets. Searching for mirror particles can be done in terms of "displaced vertices".

\section{Does the right-handed neutrino contribute to the total energy density? Is it constrained by experimental neutrino masses?}

It is not the case in this model because $\nu_{R}$ 's can decay into $\nu_{L}$ 's and $\phi_{S}$ 's. This implies that all the $\nu_{R}$ 's "born" today in astrophysical processes also decay quickly and thus, do not contribute to the total energy density of the Universe and are not constrained by cosmological data.

\section{Does this model have a 'dark matter' candidate?}

Since the $\nu_{R}$ 's are non-sterile they cannot become dark matter candidates. The only particles in this model that seem to behave as the dark matter are Higgs singlets. With the mass around $\mathcal{O}(100 \mathrm{MeV})$, they would become 'cold dark matters'.

All in all, a model of neutrino masses and mixings is such an interesting model which not only provides reasonable explanations for current phenomena but also leads us into some important aspects in future research. 


\section{Appendix A}

\section{Effective Lagrangian For $\mu-e$ Conversion}

Effective Lagrangian is a powerful technique to analyze low energy processes like $\mu \rightarrow e$ conversion in nuclei since the momentum transfer $q^{2}$ is typically of the order $\mathcal{O}\left(m_{\mu}^{2}\right) \ll m_{N}^{2}$ for nucleus $N$. The most general CLFV effective Lagrangian which contributes to the $\mu-e$ conversion in nuclei has been studied by various groups $112,113,123$. At the scale $\Lambda$ where the heavy particles (including particles beyond the SM as well as the heavy top, bottom and charm quarks) being integrated out, the relevant terms for the model we are studying are

$$
\begin{aligned}
\mathcal{L}_{\mathrm{eff}}= & -\frac{1}{\Lambda^{2}}\left[\left(C_{D R} m_{\mu} \bar{e} \sigma^{\alpha \beta} P_{L} \mu+C_{D L} m_{\mu} \bar{e} \sigma^{\alpha \beta} P_{R} \mu\right) F_{\alpha \beta}\right. \\
& \left.+\sum_{q=u, d, s}\left(C_{V R}^{(q)} \bar{e} \gamma^{\alpha} P_{R} \mu+C_{V L}^{(q)} \bar{e} \gamma^{\alpha} P_{L} \mu\right) \bar{q} \gamma_{\alpha} q+\text { H.c. }\right] .
\end{aligned}
$$

Here $m_{\mu}$ is the muon mass; $P_{L, R}=\left(1 \mp \gamma_{5}\right) / 2, \sigma_{\mu \nu}=i\left[\gamma_{\mu}, \gamma_{\nu}\right] / 2 ; F_{\alpha \beta}$ is the electromagnetic field strength; finally, $C_{D(L, R)}$ and $C_{V(L, R)}^{(q)}$ are dimensionless coupling constants depending on specific LFV model. In the specific mirror model calculation, we will be focusing on the photon and $Z$ boson exchange diagrams which contribute only to the magnetic and electric dipole moment operators as well as the vector and axial vector lepton bilinears.

To determine the conversion rate, the above effective Lagrangian A.1 is needed to scale down to the nuclear scale where the hadronic matrix elements $\left\langle N\left|\bar{q} \gamma_{\mu} q\right| N\right\rangle,\left\langle N\left|F^{\alpha \beta} F_{\alpha \beta}\right| N\right\rangle$ are evaluated. In addition, the muon and electron wave functions may be significantly deviated from 
Table A.1: Values of the dimensionless overlap integrals for aluminum, titanium and gold, evaluated under the assumption that the proton and neutron distributions within each nuclei are the same [113.

\begin{tabular}{lccc}
\hline Nucleus & $D$ & $V^{(p)}$ & $V^{(n)}$ \\
\hline \hline${ }_{13}^{27} \mathrm{Al}$ & 0.0362 & 0.0161 & 0.0173 \\
${ }_{22}^{48} \mathrm{Ti}$ & 0.0864 & 0.0396 & 0.0468 \\
${ }_{79}{ }^{197} \mathrm{Au}$ & 0.189 & 0.0974 & 0.146 \\
\hline
\end{tabular}

plane wave due to distortion by the coulomb potential of the nuclei. For high $Z$ nuclei, relativistic corrections to their wave functions are important as well. The formula for the conversion rate is given by 113,123

$$
\Gamma_{\mathrm{conv}}=\frac{m_{\mu}^{5}}{4 \Lambda^{4}}\left(\left|C_{D R} D+4 \tilde{C}_{V R}^{(p)} V^{(p)}+4 \tilde{C}_{V R}^{(n)} V^{(n)}\right|^{2}+\left|C_{D L} D+4 \tilde{C}_{V L}^{(p)} V^{(p)}+4 \tilde{C}_{V L}^{(n)} V^{(n)}\right|^{2}\right) .
$$

In Eq. A.2 the coupling constants $\tilde{C}_{V(R, L)}^{(p, n)}$ are defined as 123

$$
\begin{aligned}
\tilde{C}_{V R}^{(p)} & =\sum_{q=u, d, s} C_{V R}^{(q)} f_{V p}^{(q)}, \\
\tilde{C}_{V R}^{(n)} & =\sum_{q=u, d, s} C_{V R}^{(q)} f_{V n}^{(q)}, \\
\tilde{C}_{V L}^{(p)} & =\sum_{q=u, d, s} C_{V L}^{(q)} f_{V p}^{(q)}, \\
\tilde{C}_{V L}^{(n)} & =\sum_{q=u, d, s} C_{V L}^{(q)} f_{V n}^{(q)},
\end{aligned}
$$

where $f_{V p}^{(q)}$ and $f_{V n}^{(q)}$ are the known nucleon vector form factors

$$
\begin{aligned}
& f_{V p}^{(u)}=2, \quad f_{V p}^{(d)}=1, \quad f_{V p}^{(s)}=0, \\
& f_{V n}^{(u)}=1, \quad f_{V n}^{(d)}=2, \quad f_{V n}^{(s)}=0 .
\end{aligned}
$$

The dimensionless quantities $D$ and $V^{(p, n)}$ in Eq. A.2 are the overlap integrals of the relativistic wave functions of muon and electron in the electric field of the nucleus weighted by appropriate combinations of proton and neutron densities [113]. Their values for the four nuclei aluminum, titanium, gold and lead are listed in Table A.1 for reference. 
Table A.2: Standard model values of the capture rates for aluminum, titanium and gold in unit of $10^{6} \mathrm{~s}^{-1}$ taken from Ref. [124.

\begin{tabular}{lc}
\hline Nucleus & $\Gamma_{\text {capt }}\left(10^{6} \mathrm{~s}^{-1}\right)$ \\
\hline \hline${ }_{13}^{27} \mathrm{Al}$ & 0.7054 \\
${ }_{22}^{48} \mathrm{Ti}$ & 2.59 \\
${ }_{79} \mathrm{Au}$ & 13.07 \\
\hline
\end{tabular}

The $\mu-e$ conversion branching ratio is defined as

$$
B_{\mu N \rightarrow e N}(Z, A) \equiv \frac{\Gamma_{\mathrm{conv}}}{\Gamma_{\mathrm{capt}}}
$$

where $\Gamma_{\text {conv }}$ is given by Eq. A.2 and $\Gamma_{\text {capt }}$ is the standard model muon capture rate. The SM capture rates for aluminum, titanium and gold have been determined experimentally [124 and they are listed in Table $\mathrm{A}$ for convenience. 


\section{Appendix B}

Formulas for $\mathcal{I}, \mathcal{J}, \mathcal{I}_{i 0}(i=1, \cdots, 5)$

For the general case of retaining the external fermion masses $m_{i, j}$, the integrals $\mathcal{I}\left(\frac{m_{\phi_{k S}}^{2}}{m_{l_{m}^{M}}^{2}}\right)$ and $\mathcal{J}\left(\frac{m_{\phi_{k S}}^{2}}{m_{l_{m}^{M}}^{2}}\right)$ in Eqs. 6.17)- 6.18 have to be replaced by $\mathcal{I}\left(\frac{m_{\phi_{k S}}^{2}}{m_{l_{m}^{M}}^{2}}, \frac{m_{i}^{2}}{m_{l_{m}^{M}}^{2}}, \frac{m_{j}^{2}}{m_{l_{m}^{M}}^{2}}\right)$ and $\mathcal{J}\left(\frac{m_{\phi_{k S}}^{2}}{m_{l_{m}^{M}}^{2}}, \frac{m_{i}^{2}}{m_{l_{m}^{M}}^{2}}, \frac{m_{j}^{2}}{m_{l_{m}^{M}}^{2}}\right)$ respectively, where

$$
\begin{aligned}
\mathcal{I}\left(r, r_{i}, r_{j}\right) & =\int_{0}^{1} d x \int_{0}^{1-x} d y \frac{x(1-x-y)}{x+y+(1-x-y)\left(r-x r_{j}-y r_{i}\right)-i 0^{+}}, \\
\mathcal{J}\left(r, r_{i}, r_{j}\right) & =\int_{0}^{1} d x \int_{0}^{1-x} d y \frac{x+y}{x+y+(1-x-y)\left(r-x r_{j}-y r_{i}\right)-i 0^{+}} .
\end{aligned}
$$

In the limit of zero momentum transfer, the Feynman parameterization integrals in the various form factors can be carried out analytically. We collect their results here.

$$
\begin{aligned}
\mathcal{I}(r) & =\int_{0}^{1} d z \int_{0}^{1-z} d x \frac{x(1-x-z)}{(1-z+z r)^{2}} \\
& =\frac{1}{12} \frac{1}{(1-r)^{4}}\left[-6 r^{2} \log r+\left(2 r^{2}+3 r-6\right) r+1\right],
\end{aligned}
$$

$\lim _{r \rightarrow 0} \mathcal{I}(r)=\frac{1}{12}$ and $\lim _{r \rightarrow 1} \mathcal{I}(r)=\frac{1}{24}$

$$
\begin{aligned}
\mathcal{J}(r) & =\int_{0}^{1} d z \int_{0}^{1-z} d x \frac{x+z}{(1-z+z r)^{2}} \\
& =\frac{1}{2(1-r)^{3}}\left[-2 r^{2} \log r+r(3 r-4)+1\right]
\end{aligned}
$$


$\lim _{r \rightarrow 0} \mathcal{J}(r)=\frac{1}{12}$ and $\lim _{r \rightarrow 1} \mathcal{J}(r)=\frac{1}{24}$.

$$
\begin{aligned}
\mathcal{I}_{10}(r) & =\int_{0}^{1} d z \int_{0}^{1-z} d x \frac{x z^{2}(1-x-z)}{(1-z+z r)^{2}} \\
& =\frac{1}{72} \frac{1}{(1-r)^{6}}\left[-12 r^{2}(3+2 r) \log r+(r-1)\left(3 r^{3}+47 r^{2}+11 r-1\right)\right],
\end{aligned}
$$

$\lim _{r \rightarrow 0} \mathcal{I}_{10}(r)=\frac{1}{72}$ and $\lim _{r \rightarrow 1} \mathcal{I}_{10}(r)=\frac{1}{360}$.

$$
\begin{aligned}
\mathcal{I}_{20}(r) & =\int_{0}^{1} d z \int_{0}^{1-z} d x \frac{x z(1-x-z)}{(1-z+z r)^{2}} \\
& =\frac{1}{36} \frac{1}{(1-r)^{5}}\left[-6 r^{2}(3+r) \log r+(r-1)\left(17 r^{2}+8 r-1\right)\right]
\end{aligned}
$$

$\lim _{r \rightarrow 0} \mathcal{I}_{20}(r)=\frac{1}{36}$ and $\lim _{r \rightarrow 1} \mathcal{I}_{20}(r)=\frac{1}{120}$.

$$
\begin{aligned}
\mathcal{I}_{30}(r) & =\int_{0}^{1} d z \int_{0}^{1-z} d x \frac{x(1-x-z)}{1-z+z r} \\
& =\frac{1}{36} \frac{1}{(1-r)^{4}}\left[6 r^{3} \log r+\left(-11 r^{2}+18 r-9\right) r+2\right]
\end{aligned}
$$

$\lim _{r \rightarrow 0} \mathcal{I}_{30}(r)=\frac{1}{18}$ and $\lim _{r \rightarrow 1} \mathcal{I}_{30}(r)=\frac{1}{24}$.

$$
\begin{aligned}
\mathcal{I}_{40}(r) & =\int_{0}^{1} d z \int_{0}^{1-z} d x \frac{x^{2} z(1-x-z)}{(1-z+z r)^{2}} \\
& =\frac{1}{144} \frac{1}{(1-r)^{6}}\left[12 r^{3}(r+4) \log r-\left(r^{2}-1\right)\left(37 r^{2}-8 r+1\right)\right]
\end{aligned}
$$

$\lim _{r \rightarrow 0} \mathcal{I}_{40}(r)=\frac{1}{144}$ and $\lim _{r \rightarrow 1} \mathcal{I}_{40}(r)=\frac{1}{360}$.

$$
\begin{aligned}
\mathcal{I}_{50}(r) & =\int_{0}^{1} d z \int_{0}^{1-z} d x \frac{x(1-z)(1-x-z)}{(1-z+z r)^{2}} \\
& =\frac{1}{18} \frac{1}{(1-r)^{5}}\left[12 r^{3} \log r-\left(3 r^{4}+10 r^{3}-18 r^{2}+6 r\right)+1\right],
\end{aligned}
$$

$\lim _{r \rightarrow 0} \mathcal{I}_{50}(r)=\frac{1}{18}$ and $\lim _{r \rightarrow 1} \mathcal{I}_{50}(r)=\frac{1}{30}$. 
Here $r$ denotes the mass ratio $m^{2} / M^{2}$, where $m$ and $M$ are the masses of the scalar singlet and mirror lepton respectively. 


\section{Appendix $\mathrm{C}$}

\section{Four-Fermion Coupling Constants $C_{V(L, R)}^{(q)}$}

The total contribution to $C_{V(L, R)}^{(q)}$ is given by

$$
C_{V(L, R)}^{(q)}=C_{V(L, R) V}^{(q) \gamma}+C_{V(L, R)}^{(q) Z}+C_{V(L, R)}^{(q) H(\mathrm{Box})}
$$

We have already derived $C_{V(L, R) V}^{(q) \gamma}$ in section 6.2.1.2. In this appendix, we show our work on finding the $C_{V(L, R)}^{(q) Z}$ from $Z$ exchange and the $C_{V(L, R)}^{(q) H(\text { Box })}$ from scalar Higgs (box) exchange. Furthermore, a discussion on how the latter 2 coupling constants are negligible compared with the former one is also included.

\section{C.1 $Z$ Boson Exchange}

For the $Z$ boson contribution in Fig. C.1, in the limit of $\left|q^{2}\right| \ll m_{Z}^{2}$, we obtain the following amplitude

$$
\begin{aligned}
\mathcal{M}_{Z} \approx \frac{G_{F}}{\sqrt{2}} & {\left[f_{L}^{Z} \overline{u_{L e}}\left(p^{\prime}\right) \gamma_{\mu} u_{L \mu}(p)+f_{R}^{Z} \overline{u_{R e}}\left(p^{\prime}\right) \gamma_{\mu} u_{R \mu}(p)\right] } \\
& \times\left[\overline{u_{q}}\left(k^{\prime}\right)\left(C_{V}^{q} \gamma^{\mu}+C_{A}^{q} \gamma^{\mu} \gamma_{5}\right) u_{q}(k)\right],
\end{aligned}
$$


where $f_{L, R}^{Z}$ are form factors given by

$$
\begin{aligned}
f_{L}^{Z}\left(q^{2}\right)= & \frac{1}{2 \pi^{2}} \sum_{k, m} \int_{0}^{1} d x \int_{0}^{1-x} d y \\
\times & \left\{\left[C_{L}^{l} \log \left(\frac{\Delta_{k m}\left(q^{2}\right)}{\Delta_{k m}(0)}\right)-C_{R}^{l} M_{m}^{2}\left(\Delta_{k m}^{-1}\left(q^{2}\right)-\Delta_{k m}^{-1}(0)\right)\right] \times \mathcal{U}_{1 m}^{L k}\left(\mathcal{U}_{2 m}^{L k}\right)^{*}\right. \\
& -m_{\mu} m_{e}(1-x-y)^{2}\left(\Delta_{k m}^{-1}\left(q^{2}\right)-\Delta_{k m}^{-1}(0)\right) \times C_{R}^{l} \mathcal{U}_{1 m}^{R k}\left(\mathcal{U}_{2 m}^{R k}\right)^{*} \\
& -M_{m}(1-x-y)\left(\Delta_{k m}^{-1}\left(q^{2}\right)-\Delta_{k m}^{-1}(0)\right) \times C_{R}^{l}\left(m_{\mu} \mathcal{U}_{1 m}^{L k}\left(\mathcal{U}_{2 m}^{R k}\right)^{*}+m_{e} \mathcal{U}_{1 m}^{R k}\left(\mathcal{U}_{2 m}^{L k}\right)^{*}\right) \\
& \left.-\frac{x y q^{2}}{\Delta_{k m}\left(q^{2}\right)} \times C_{L}^{l} \mathcal{U}_{1 m}^{L k}\left(\mathcal{U}_{2 m}^{L k}\right)^{*}\right\} \\
+ & \frac{1}{2 \pi^{2}} \frac{\left(C_{L}^{l}-C_{R}^{l}\right)}{\left(m_{\mu}^{2}-m_{e}^{2}\right)}\left\{\sum_{k, m} \int_{0}^{1} d x \log \left(\frac{\Delta_{k m}^{e}(x)}{\Delta_{k m}^{\mu}(x)}\right) \times\left[\frac{M_{m}^{2}}{1-x} \mathcal{U}_{1 m}^{L k}\left(\mathcal{U}_{2 m}^{L k}\right)^{*}\right.\right. \\
& \left.\left.+m_{\mu} m_{e}(1-x) \mathcal{U}_{1 m}^{R k}\left(\mathcal{U}_{2 m}^{R k}\right)^{*}+M_{m}\left(m_{\mu} \mathcal{U}_{1 m}^{L k}\left(\mathcal{U}_{2 m}^{R k}\right)^{*}+m_{e} \mathcal{U}_{1 m}^{R k}\left(\mathcal{U}_{2 m}^{L k}\right)^{*}\right)\right]\right\} \quad(\mathrm{C} .3)
\end{aligned}
$$

and $f_{R}^{Z}\left(q^{2}\right)$ can be obtained from $f_{L}^{Z}\left(q^{2}\right)$ in Eq. C.3 with $L \leftrightarrow R$ for all the quantities with $L, R$ subscripts or superscripts. Here $C_{L}^{f}=T^{3}(f)-Q_{f} \sin ^{2} \theta_{W}$ and $C_{R}^{f}=-Q_{f} \sin ^{2} \theta_{W}$ are the chiral couplings of fermion $f$ with the $Z$ boson. We have used the fact that for muon, electron and mirror charged leptons they all have the same $C_{L, R}^{l} \cdot \Delta_{k m}\left(q^{2}\right)$ in Eq. (C.2) is given by Eq. 6.42 and $\Delta_{k m}^{\mu, e}(x)$ is given by

$$
\Delta_{k m}^{\mu, e}(x)=x M_{m}^{2}+(1-x) m_{k}^{2}-x(1-x) m_{e, \mu}^{2} .
$$
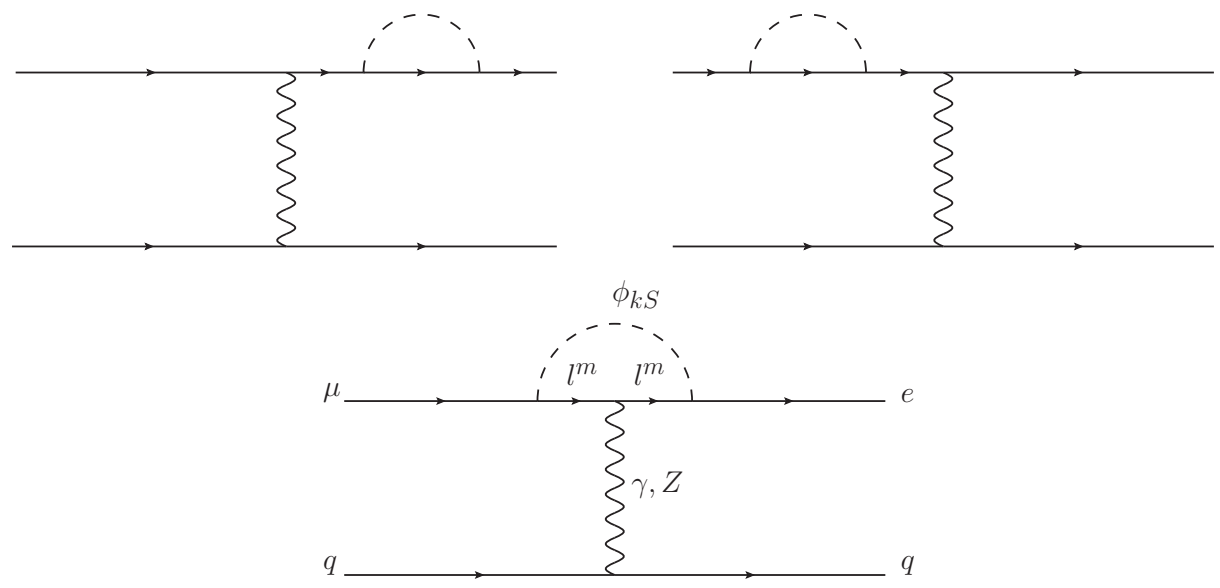

Figure C.1: One-loop induced Feynman diagrams from photon and $Z$ boson exchanges for $\mu \rightarrow e$ conversion in EW-scale $\nu_{R}$ model. 
In derivation of Eq. C.2, we have dropped terms proportional to $q_{\mu}$ and $i \sigma_{\mu \nu} q^{\nu}$ from the $Z$ vertex diagram in Fig. C.1. The $q_{\mu}$ term when multiples the quark current $\bar{q} \gamma^{\mu}\left(C_{V}^{q}+C_{A}^{q} \gamma_{5}\right) q$ and invokes the free quark equation of motion will give zero in the vector part, while for the axial vector part it will produce term proportional to the light quark mass. The $i \sigma_{\mu \nu} q^{\nu}$ term will give rise to dimension-7 four-fermion operators with one derivative in the position space. Their contributions will be suppressed by $\mathcal{O}\left(m_{\mu} / M\right)$ where $M$ is the heavy mirror lepton mass scale inside the loop as compared with the dimension 6 four-fermion operators. We will ignore these two terms in our analysis for $\mu-e$ conversion.

At $q^{2}=0$, we note that $f_{L, R}^{Z} \neq 0$. For small $q^{2}$, the factors of $\log \left(\Delta_{k m}\left(q^{2}\right) / \Delta_{k m}(0)\right)$ and $\Delta_{k m}^{-1}\left(q^{2}\right)-\Delta_{k m}^{-1}(0)$ in Eq. C.3 can be approximated by using

$$
\begin{aligned}
\log \left(\frac{\Delta_{k m}\left(q^{2}\right)}{\Delta_{k m}(0)}\right) & \approx-\frac{x y q^{2}}{\Delta_{k m}(0)}, \\
\Delta_{k m}^{-1}\left(q^{2}\right)-\Delta_{k m}^{-1}(0) & \approx+\frac{x y q^{2}}{\left(\Delta_{k m}(0)\right)^{2}} .
\end{aligned}
$$

For practical purpose, we will evaluate $q^{2}=-m_{\mu}^{2}$. The amplitude $\mathcal{M}_{Z}$ in Eq. (C.2) can be reproduced by the following Fermi interaction

$$
\mathcal{L}_{Z, \mathrm{eff}}=\frac{G_{F}}{\sqrt{2}}\left[f_{L}^{Z}\left(-m_{\mu}^{2}\right) \overline{e_{L}} \gamma_{\mu} \mu_{L}+f_{R}^{Z}\left(-m_{\mu}^{2}\right) \overline{e_{R}} \gamma_{\mu} \mu_{R}\right]\left[\bar{q}\left(C_{V}^{q} \gamma^{\mu}+C_{A}^{q} \gamma^{\mu} \gamma_{5}\right) q\right]+\cdots
$$

where the "..." denotes non-local operators. Once again, matching Eq. (C.6) with the second line of Eq. A.1), we obtain

$$
\frac{C_{V(L, R)}^{(q) Z}}{\Lambda^{2}}=-\frac{G_{F}}{\sqrt{2}} C_{V}^{q} f_{L, R}^{Z}\left(-m_{\mu}^{2}\right)
$$

As a bonus, we also obtain the effective axial vector coupling

$$
\frac{C_{A(L, R)}^{(q) Z}}{\Lambda^{2}}=-\frac{G_{F}}{\sqrt{2}} C_{A}^{q} f_{L, R}^{Z}\left(-m_{\mu}^{2}\right),
$$

which is nevertheless irrelevant for the coherent $\mu-e$ conversion processes in nuclei. 


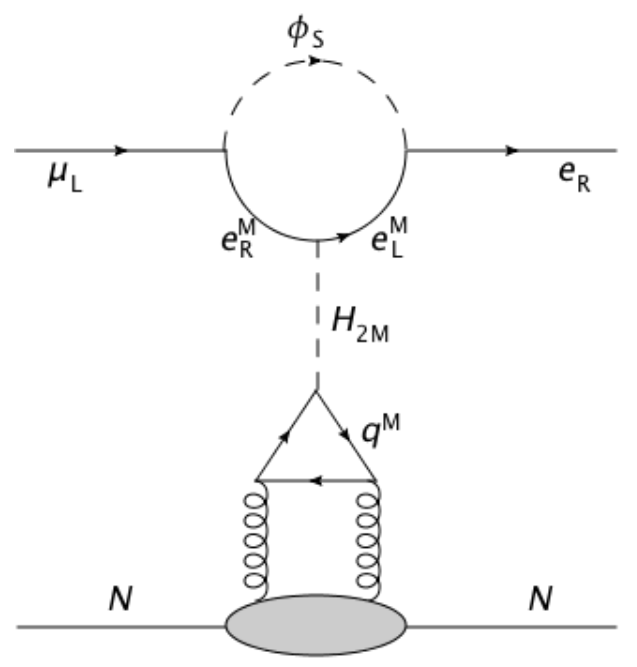

Figure C.2: One-loop induced Feynman diagrams Higgs exchange with heavy quarks and mirror quarks for $\mu \rightarrow e$ conversion in EW-scale $\nu_{R}$ model.

\section{C.2 Scalar Higgs Exchange}

The discussion will based upon two papers 125 and 73 .

From Fig. C.2 since only mirror fermions are involved, we looked at the couplings for Table 5 of the paper 73. We first write down the Lagrangian for an interaction of the scalar Higgs to the nucleus via a triangle diagram as shown in Fig. (C.2.

$$
\mathcal{L}_{H}=-\frac{g}{2 m_{W} s_{2 M}} H_{2 M} \sum\left(m_{q_{M}} \bar{q}_{M} q_{M}+m_{l_{M}} \bar{l}_{M} l_{M}\right)
$$

where $s_{2 M}=v_{2 M} / v$. Without the trace anomaly, one has the trace of symmetric energy-momentum tensor as

$$
\Theta_{\mu}^{\mu}=m_{u} \bar{u} u+m_{d} \bar{d} d+m_{s} \bar{s} s+\sum_{k} m_{h} \bar{h} h, \quad k=c, b, t, q_{M}, l_{M} .
$$

With the trace anomaly coming from the renormalization, one has

$$
\Theta_{\mu}^{\mu}=\frac{\beta\left(\alpha_{S}\right)}{4 \alpha_{S}} G_{\mu \nu}^{a} G^{\mu \nu, a}+\cdots
$$

with its $\beta$-function

$$
\beta\left(\alpha_{S}\right)=\left(\frac{2}{3} n_{h}-9\right) \frac{\alpha_{S}^{2}}{2 \pi}+\mathcal{O}\left(\alpha_{S}^{3}\right)
$$


As discussed by Shifman, Vainshtein, and Zakharov [125, the valence quarks are not the heavy quarks and they enter through the triangle diagram. This results in

$$
\sum m_{h} \bar{h} h \rightarrow-\frac{2}{3} n_{h} \frac{\alpha_{S}}{8 \pi} G_{\mu \nu}^{a} G^{\mu \nu, a}+\cdots
$$

Therefore the overall $\beta$-function is

$$
\tilde{\beta}\left(\alpha_{S}\right)=-\frac{9 \alpha_{S}^{2}}{2 \pi}+\cdots
$$

Notice that in chiral limit which sets $m_{u}, m_{d}, m_{s} \rightarrow 0$,

$$
\sum m_{h} \bar{h} h \rightarrow-\frac{2}{3} \frac{\alpha_{S}}{8 \pi} n_{h} G_{\mu \nu}^{a} G^{\mu \nu, a}
$$

Hence we obtain the amplitude of the interaction via the triangle diagram presented in Fig. (C.2) as follows

$$
\begin{aligned}
& \frac{g}{2 m_{W} s_{2 M}} H_{2 M}\left\langle N\left|\sum m_{q^{M}} \bar{q}^{M} q^{M}\right| N\right\rangle \\
= & \frac{g}{2 m_{W} s_{2 M}} H_{2 M}\left(-\frac{2}{3} n_{h^{M}}\right)\left\langle N\left|\frac{\alpha_{S}}{8 \pi} G_{\mu \nu}^{a} G^{\mu \nu, a}\right| N\right\rangle .
\end{aligned}
$$

Again, in the chiral limit, the term

$$
\begin{aligned}
\left\langle N\left|\Theta_{\mu}^{\mu}\right| N\right\rangle & \simeq\left\langle N\left|\frac{\tilde{\beta}\left(\alpha_{S}\right)}{4 \alpha_{S}} G_{\mu \nu}^{a} G^{\mu \nu, a}\right| N\right\rangle \\
& \simeq\left\langle N\left|-\frac{9 \alpha_{S}^{2}}{2 \pi}\right| N\right\rangle .
\end{aligned}
$$

$\mathrm{Or}$

$$
\left\langle N\left|\frac{\alpha_{S}^{2}}{2 \pi}\right| N\right\rangle \simeq-\frac{1}{9}\left\langle N\left|\Theta_{\mu}^{\mu}\right| N\right\rangle
$$

Comparing Eqs. C.16) and C.17 the amplitude of the scalar Higgs-nucleus interaction is given by

$$
\left(\frac{g}{2 m_{W} s_{2 M}}\right)\left(\frac{2 n_{h^{M}} m_{N}}{27}\right) H_{2 M} \overline{\psi_{N}} \psi_{N}
$$

where $\left\langle N\left|\Theta_{\mu}^{\mu}\right| N\right\rangle=m_{N} \overline{\psi_{N}} \psi_{N}, m_{N}$ is the nucleus mass. On the other hand, the EW- $\nu_{R}$ model yields $n_{h^{M}}=6$ so Eq. C.18 becomes

$$
\left(\frac{g}{2 m_{W} s_{2 M}}\right)\left(\frac{12 m_{N}}{27}\right) H_{2 M}{\overline{\psi_{N}}}_{\psi_{N}} .
$$


All in all. the diagram C.2 gives

$$
\bar{e}_{R} \mu_{L} I\left(q^{2}\right) \frac{12}{27}\left(\frac{g}{2 m_{W} s_{2 M}}\right)^{2}\left(m_{l^{M}} m_{N}\right) \frac{1}{q^{2}-m_{H_{2 M}}^{2}} \bar{\psi}_{N} \psi_{N} .
$$

where $I\left(q^{2}\right)$ is the loop integral. However the above expression has to be rewritten in terms of the Higgs mass eigenstates. In the paper 73 , we found the following scenarios

(1) Dr. Jekyll scenario in which $125 \mathrm{GeV}$ Higgs $\tilde{H} \sim H_{1}^{0}$ SM-like gives an example

$$
\left(\begin{array}{c}
\tilde{H} \\
\tilde{H}^{\prime} \\
\tilde{H}^{\prime \prime}
\end{array}\right)=\left(\begin{array}{ccc}
0.998 & -0.0518 & -0.0329 \\
0.0514 & 0.999 & -0.014 \\
0.0336 & 0.0123 & 0.999
\end{array}\right)\left(\begin{array}{c}
H_{1}^{0} \\
H_{1 M}^{0} \\
H_{1}^{0 \prime}
\end{array}\right)
$$

(2) Mr. Hyde scenario in which $125 \mathrm{GeV}$ Higgs $\tilde{H} \sim H_{1}^{0 \prime}$ (not SM-like) gives an example

$$
\left(\begin{array}{c}
\tilde{H} \\
\tilde{H}^{\prime} \\
\tilde{H}^{\prime \prime}
\end{array}\right)=\left(\begin{array}{ccc}
0.3 & -0.094 & -0.949 \\
0.334 & -0.921 & -0.197 \\
0.893 & 0.376 & 0.246
\end{array}\right)\left(\begin{array}{c}
H_{1}^{0} \\
H_{1 M}^{0} \\
H_{1}^{0 \prime}
\end{array}\right)
$$

In examples of the above two different scenarios, we can see that the $125 \mathrm{GeV}$ Higgs is not the proper exchanged scalar since the coefficients of coupling between $\tilde{H}$ and $H_{1 M}^{0}$ are small. Therefore, it has to be the next heavier one $\tilde{H}^{\prime}$ having a mass $\sim 400 \mathrm{GeV} 73$.

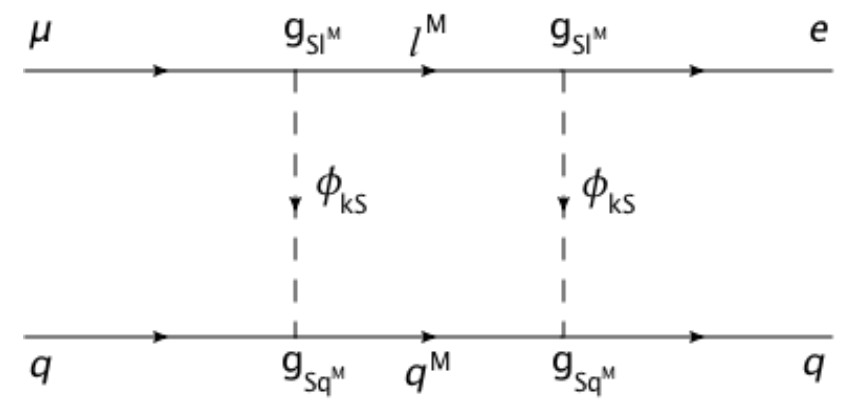

Figure C.3: Box diagram for $\mu \rightarrow e$ conversion in EW-scale $\nu_{R}$ model. 
In conclusion, the contribution of scalar Higgs exchange to the total four-fermion coupling constant in Eq. (C.1) is suppressed by the inverse of heavy Higgs masses and can be considered negligible in comparison with that of the photon exchange.

\section{C.3 Box diagram contribution}

In order to prove the negligibility of the box diagram we will not go into detailed calculations but make such a hand-waving argument instead. From Fig. (C.3) the amplitude of the diagram will

directly proportional to $\left(g_{S l^{M}}\right)^{2}\left(g_{S q^{M}}\right)^{2}$. We have learned from the numerical analysis presented in section 6.2.3 that the Yukawa couplings $g_{S l^{M}}$ are of $\mathcal{O}\left(10^{-5}\right)$. These pieces make the total amplitude become very small and therefore, we will not have to worry about the effects of the box diagram in the $\mu-e$ conversion decay rate. 


\section{Bibliography}

[1] Wikipedia, https://en.wikipedia.org/wiki/Fermi-Dirac_statistics

[2] Wikipedia, https://en.wikipedia.org/wiki/Bose-Einstein_statistics

[3] C. Patrignani et al. (Particle Data Group), Chin. Phys. C, 40, 100001 (2016).

[4] G. Aad et al. [ATLAS Collaboration], "Observation of a new particle in the search for the Standard Model Higgs boson with the ATLAS detector at the LHC," Phys. Lett. B 716, 1 (2012) [arXiv:1207.7214 [hep-ex]].

[5] S. Chatrchyan et al. [CMS Collaboration], "Observation of a new boson at a mass of 125 GeV with the CMS experiment at the LHC," Phys. Lett. B 716, 30 (2012) [arXiv:1207.7235 $[$ hep-ex]].

[6] S. Chatrchyan et al. [CMS Collaboration], "Evidence for the direct decay of the $125 \mathrm{GeV}$ Higgs boson to fermions," Nature Phys. 10, 557 (2014) [arXiv:1401.6527 [hep-ex]].

[7] T. P. Cheng, L. F. Li, "Gauge theory of elementary particle physics", Clarendon Press, 1984.

[8] Wikipedia, https://en.wikipedia.org/wiki/Gell-Mann-Nishijima_formula

[9] J. Beringer et al. [Particle Data Group], "Review of Particle Physics (RPP)," Phys. Rev. D 86, 010001 (2012).

[10] J. Bernstein, "Spontaneous symmetry breaking, gauge theories, the higgs mechanism and all that," Rev. Mod. Phys. 46, 7 (1974). 
[11] M. Jacob, G.C. Wick, "On the general theory of collisions for particles with spin", Annals of Physics, Volume 7, Issue 4, 1959, Pages 404-428, ISSN 0003-4916, doi.org/10.1016/00034916(59)90051-X.

[12] Wikipedia, https://en.wikipedia.org/wiki/

[13] Peter W. Higgs, "Broken symmetries, massless particles and gauge fields", Phys. Lett., 12:132133 (1964).

[14] Peter W. Higgs, "Broken symmetries and the masses of gauge boson", Phys. Rev. Lett., 13:508-509 (1964).

[15] F. Englert and R. Brout, "Broken symmetry and the mass of gauge vector mesons", Phys. Rev. Lett., 13:321-323 (1964).

[16] G. S. Guralnik, C. R. Hagen, and T. W. B. Kibble, "Global conservation laws and massless particles", Phys. Rev. Lett., 13:585-587 (1964).

[17] E158 Experiment, SLAC, http://www.slac.stanford.edu/exp/e158/; Q-weak Experiment, Jefferson Lab, https://www.jlab.org/qweak/.

[18] N. Cabibbo, "Unitary Symmetry and Leptonic Decays," Phys. Rev. Lett. 10, 531 (1963). doi:10.1103/PhysRevLett.10.531

[19] M. Kobayashi and T. Maskawa, "CP Violation in the Renormalizable Theory of Weak Interaction," Prog. Theor. Phys. 49, 652 (1973). doi:10.1143/PTP.49.652

[20] T. Gershon, "Overview of the CKM Matrix," Pramana 79, 1091 (2012) doi:10.1007/s12043012-0418-y [arXiv:1112.1984 [hep-ex]].

[21] Particle Data Group, "The CKM Quark-Mixing Matrix", http://pdg.lbl.gov/2016/reviews/rpp2016-rev-ckm-matrix.pdf 
[22] L. Wolfenstein, "Parametrization of the Kobayashi-Maskawa Matrix," Phys. Rev. Lett. 51, 1945 (1983).

[23] M. Bona et al. [UTfit Collab.], JHEP 507, 28 (2005) [hep-ph/0501199], and updates at http://www.utfit.org/.

[24] M. Bona et al. [UTfit Collab.], JHEP 0803, 049 (2008) [arXiv:0707.0636].

[25] We thank the CKMfitter and UTfit groups for performing fits and preparing plots using input values from the "CKM quark-mixing matrix" review of PDG (2016).

[26] "The Reines-Cowan Experiments: Detecting the Poltergerist", Los Alamos Science, 25: 3, (1997).

[27] C. L. Cowan Jr., F. Reines, F. B. Harrison, H. W. Kruse, A. D. McGuide, "Detection of the Free Neutrino: a Confirmation", Science, 124 (3212): 103-4, (1956) doi:10.1126/science.124.3212.103

[28] G. Danby, J. M. Gaillard, K. A. Goulianos, L. M. Lederman, N. B. Mistry, M. Schwartz and J. Steinberger, "Observation of High-Energy Neutrino Reactions and the Existence of Two Kinds of Neutrinos," Phys. Rev. Lett. 9, 36 (1962). doi:10.1103/PhysRevLett.9.36

[29] M. L. Perl et al., "Evidence for Anomalous Lepton Production in e+ - e- Annihilation," Phys. Rev. Lett. 35, 1489 (1975). doi:10.1103/PhysRevLett.35.1489

[30] K. Kodama et al. [DONUT Collaboration], "Observation of tau neutrino interactions," Phys. Lett. B 504, 218 (2001) doi:10.1016/S0370-2693(01)00307-0 [hep-ex/0012035].

[31] "Physicists Find First Direct Evidence for Tau Neutrino at Fermilab" (Press release), Fermilab (2000).

[32] G. W. Rodeback and J. S. Allen, "Neutrino recoils following the capture of orbital electrons in A-37," Camb. Monogr. Part. Phys. Nucl. Phys. Cosmol. 14, 28 (2000) [Phys. Rev. 86, 446 (1952)]. doi:10.1103/PhysRev.86.446 
[33] Press Release, https://www.nobelprize.org/nobel_ prizes/physics/laureates/2015/press.html, October 2015.

[34] Solar Neutrino Experiments, https://www.bnl.gov/bnlweb/raydavis/research.html.

[35] B. T. Cleveland, T. Daily, R. Davis, Jr., J. R. Distel, K. Lande, C. K. Lee, P. S. Wildenhain and J. Ullman, "Measurement of the solar electron neutrino flux with the Homestake chlorine detector," Astrophys. J. 496, 505 (1998). doi:10.1086/305343

[36] E. Kearns, T. Kajita and Y. Totsuka, "Detecting Massive Neutrinos", Scientific American, August 1999.

[37] J. N. Bahcall, "Gallium solar neutrino experiments: Absorption cross-sections, neutrino spectra, and predicted event rates," Phys. Rev. C 56, 3391 (1997) [hep-ph/9710491].

[38] T. Kajita, "Atmospheric Neutrinos And Discovery Of Neutrino Oscillations," Proc. Japan Acad. B 86, 303 (2010). doi:10.2183/pjab.86.303

[39] D. H. Perkins, "The Atmospheric neutrino problem: A Critique," Nucl. Phys. B 399, 3 (1993). doi:10.1016/0550-3213(93)90613-T

[40] T. Kajita [Super-Kamiokande and Kamiokande Collaborations], "Atmospheric neutrino results from Super-Kamiokande and Kamiokande: Evidence for neutrino(mu) oscillations," Nucl. Phys. Proc. Suppl. 77, 123 (1999) [hep-ex/9810001].

[41] K. Nakamura, S. T. Petcov, "Neutrino mass, mixing, and oscillations review", Particle Data Group, 2016, http://pdg.lbl.gov/2016/reviews/rpp2016-rev-neutrino-mixing.pdf.

[42] D. Karlen in RPP2012, Phys. Rev. D86, Part I, 629 (2012).

[43] P. A. R. Ade et al. [Planck Collaboration], "Planck 2015 results. XIII. Cosmological parameters," Astron. Astrophys. 594, A13 (2016) doi:10.1051/0004-6361/201525830 [arXiv:1502.01589 [astro-ph.CO]]. 
[44] Z. Maki, M. Nakagawa and S. Sakata, "Remarks on the unified model of elementary particles," Prog. Theor. Phys. 28, 870 (1962). doi:10.1143/PTP.28.870

[45] B. Pontecorvo, "Inverse beta processes and nonconservation of lepton charge," Sov. Phys. JETP 7, 172 (1958) [Zh. Eksp. Teor. Fiz. 34, 247 (1957)].

[46] S. F. King and C. Luhn, "Neutrino Mass and Mixing with Discrete Symmetry," Rept. Prog. Phys. 76, 056201 (2013) doi:10.1088/0034-4885/76/5/056201 [arXiv:1301.1340 [hep-ph]].

[47] D. G. Michael et al. [MINOS Collaboration], "Observation of muon neutrino disappearance with the MINOS detectors and the NuMI neutrino beam," Phys. Rev. Lett. 97, 191801 (2006) [hep-ex/0607088].

[48] P. Adamson et al. [MINOS Collaboration], "Measurement of Neutrino Oscillations with the MINOS Detectors in the NuMI Beam," Phys. Rev. Lett. 101, 131802 (2008) [arXiv:0806.2237 $[$ hep-ex]].

[49] Q.R. Ahmad et al. [SNO Collab.], Phys. Rev. Lett. 87, 071301 (2001); Q.R. Ahmad et al. [SNO Collab.], Phys. Rev. Lett. 89, 011301 (2002); B. Aharmim et al. [SNO Collab.], Phys. Rev. C72, 055502 (2005); B. Aharmim et al. [SNO Collab.], Phys. Rev. Lett. 101, 111301 (2008); Phys. Rev. C87, 015502 (2013).

[50] T. Araki et al. [KamLAND Collab.], Phys. Rev. Lett. 94, 081801 (2005); S. Abe et al. [KamLAND Collab.], Phys. Rev. Lett. 100, 221803 (2008); A. Gando et al. [KamLAND Collab.], Phys. Rev. D83, 052002 (2011).

[51] Y. Abe et al. [Double Chooz Collaboration], "Improved measurements of the neutrino mixing angle $\theta_{13}$ with the Double Chooz detector," JHEP 1410, 086 (2014) [arXiv:1406.7763 [hep$\mathrm{ex}]]$. 
[52] F. P. An et al. [Daya Bay Collaboration], "New Measurement of Antineutrino Oscillation with the Full Detector Configuration at Daya Bay," Phys. Rev. Lett. 115, no. 11, 111802 (2015) [arXiv:1505.03456 [hep-ex]].

[53] J. H. Choi et al. [RENO Collaboration], "Observation of Energy and Baseline Dependent Reactor Antineutrino Disappearance in the RENO Experiment," Phys. Rev. Lett. 116, no. 21, 211801 (2016) [arXiv:1511.05849 [hep-ex]].

[54] F. Capozzi, G. L. Fogli, E. Lisi, A. Marrone, D. Montanino and A. Palazzo, "Status of $3 \nu$ oscillation parameters at the end of 2013," J. Phys. Conf. Ser. 598, no. 1, 012002 (2015).

[55] F. Capozzi, G. L. Fogli, E. Lisi, A. Marrone, D. Montanino and A. Palazzo, "Status of threeneutrino oscillation parameters, circa 2013," Phys. Rev. D 89, 093018 (2014) [arXiv:1312.2878 [hep-ph]].

[56] S. Weinberg, "Varieties of Baryon and Lepton Nonconservation," Phys. Rev. D 22, 1694 (1980). doi:10.1103/PhysRevD.22.1694

[57] A. Faessler and F. Simkovic, "Double beta decay," J. Phys. G 24, 2139 (1998) doi:10.1088/0954-3899/24/12/001 [hep-ph/9901215].

[58] H. V. Klapdor-Kleingrothaus, "Perspectives of double beta and dark matter search as windows to new physics," Springer Tracts Mod. Phys. 163, 69 (2000) [hep-ex/9907040].

[59] S. Dell'Oro, S. Marcocci, M. Viel and F. Vissani, "Neutrinoless double beta decay: 2015 review," Adv. High Energy Phys. 2016, 2162659 (2016) doi:10.1155/2016/2162659 [arXiv:1601.07512 [hep-ph]].

[60] G. Senjanovic, "Neutrino mass: From LHC to grand unification," Riv. Nuovo Cim. 34, 1 (2011). doi:10.1393/ncr/i2011-10061-8 
[61] G. Barenboim, M. Gorbahn, U. Nierste and M. Raidal, "Higgs sector of the minimal leftright symmetric model," Phys. Rev. D 65, 095003 (2002) doi:10.1103/PhysRevD.65.095003 [hep-ph/0107121].

[62] W. Y. Keung and G. Senjanovic, "Majorana Neutrinos and the Production of the Right-handed Charged Gauge Boson," Phys. Rev. Lett. 50, 1427 (1983). doi:10.1103/PhysRevLett.50.1427

[63] V. Khachatryan et al. [CMS Collaboration], Eur. Phys. J. C 74, no. 11, 3149 (2014) doi:10.1140/epjc/s10052-014-3149-z [arXiv:1407.3683 [hep-ex]].

[64] P. Q. Hung, "A Model of electroweak-scale right-handed neutrino mass," Phys. Lett. B 649, 275 (2007) doi:10.1016/j.physletb.2007.03.067 [hep-ph/0612004].

[65] A. Aranda, J. Hernandez-Sanchez and P. Q. Hung, "Implications of the discovery of a Higgs triplet on electroweak right-handed neutrinos," JHEP 0811, 092 (2008) doi:10.1088/11266708/2008/11/092 [arXiv:0809.2791 [hep-ph]].

[66] M. S. Chanowitz and M. Golden, "Higgs Boson Triplets With $\mathrm{M}(W)=\mathrm{M}(Z) \cos \theta \omega$, , Phys. Lett. 165B, 105 (1985). doi:10.1016/0370-2693(85)90700-2

[67] H. Georgi and M. Machacek, "Doubly Charged Higgs Bosons," Nucl. Phys. B 262, 463 (1985). doi:10.1016/0550-3213(85)90325-6

[68] R. Godbole, B. Mukhopadhyaya and M. Nowakowski, "Triplet Higgs bosons at e+ e- colliders," Phys. Lett. B 352, 388 (1995) doi:10.1016/0370-2693(95)00481-Y [hep-ph/9411324].

[69] J. F. Gunion, R. Vega and J. Wudka, Phys. Rev. D 42, 1673 (1990). doi:10.1103/PhysRevD.42.1673

[70] P. Q. Hung, "A Model of electroweak-scale right-handed neutrino mass," Phys. Lett. B 649, 275 (2007) [hep-ph/0612004]; P. Q. Hung, "Implications of right-handed neutrinos 
with electroweak-scale masses," Frascati Phys. Ser. 44, 313 (2007) [arXiv:0706.2753 [hepph]]; P. Q. Hung, "Electroweak-scale mirror fermions, $\mu \rightarrow e \gamma$ and $\tau \rightarrow \mu \gamma$. " Phys. Lett. B 659, 585 (2008) [arXiv:0711.0733 [hep-ph]]; P. Q. Hung, "Consequences of Pati-Salam unification of electroweak-scale active nu(R) model: keV sterile neutrinos: four families," Nucl. Phys. B 805, 326 (2008) [arXiv:0805.3486 [hep-ph]]; A. Aranda, J. Hernandez-Sanchez and P. Q. Hung, "Implications of the discovery of a Higgs triplet on electroweak right-handed neutrinos," JHEP 0811, 092 (2008) [arXiv:0809.2791 [hep-ph]].

[71] V. Hoang, P. Q. Hung and A. S. Kamat, "Electroweak precision constraints on the electroweak-scale right-handed neutrino model," Nucl. Phys. B 877, 190 (2013) [arXiv:1303.0428 [hep-ph]].

[72] S. Chatrchyan et al. [CMS Collaboration], "On the mass and spin-parity of the Higgs boson candidate via its decays to Z boson pairs," Phys. Rev. Lett. 110, 081803 (2013) [arXiv:1212.6639 [hep-ex]].

[73] V. Hoang, P. Q. Hung and A. S. Kamat, "Non-sterile electroweak-scale right-handed neutrinos and the dual nature of the 126-GeV scalar," Nucl. Phys. B 896 (2015) 611-656, [arXiv:1412.0343 [hep-ph]].

[74] S. Chakdar, K. Ghosh, V. Hoang, P. Q. Hung and S. Nandi, "Search for mirror quarks at the LHC," Phys. Rev. D 93, no. 3, 035007 (2016) doi:10.1103/PhysRevD.93.035007 [arXiv:1508.07318 [hep-ph]].

[75] S. Chatrchyan et al. [CMS Collaboration], "Measurement of Higgs boson production and properties in the WW decay channel with leptonic final states," JHEP 1401, 096 (2014) [arXiv:1312.1129 [hep-ex]].

[76] S. Chatrchyan et al. [CMS Collaboration], "Measurement of the properties of a Higgs boson in the four-lepton final state," Phys. Rev. D 89, no. 9, 092007 (2014) [arXiv:1312.5353 [hep-ex]]. 
[77] S. Chatrchyan et al. [CMS Collaboration], "Search for the standard model Higgs boson produced in association with a $\mathrm{W}$ or a $\mathrm{Z}$ boson and decaying to bottom quarks," Phys. Rev. D 89, 012003 (2014) [arXiv:1310.3687 [hep-ex]].

[78] S. Chatrchyan et al. [CMS Collaboration], "Evidence for the $125 \mathrm{GeV}$ Higgs boson decaying to a pair of $\tau$ leptons," JHEP 1405, 104 (2014) [arXiv:1401.5041 [hep-ex]].

[79] K. Nakamura et al. [Particle Data Group Collaboration], "Review of particle physics," J. Phys. G 37, 075021 (2010).

[80] P. Minkowski, Phys. Lett. B 67, 421 (1977); M. Gell-Mann, P. Ramond and R. Slansky, in Supergravity, eds. P. van Niewenhuizen and D. Z. Freedman (North Holland 1979); T. Yanagida, in Proceeding of Workshop on Unified Theory and Baryon Number in the Universe, eds. O. Sawada and A. Sugamoto (KEK 1979); S. L. Glashow, The future of elementary particle physics, in Proceedings of the 1979 Cargese Summer Institute on quarks and leptons (M. Levy, J. -L. Basdevant, D. Speiser, J. Speiser, R. Gatsmans, and M. Jacob, eds.) Plenum Press, New York, 1980, p. 687; R. N. Mohapatra and G. Senjanovíc, Phys. Rev. Lett. 44, 912 (1980); J. Schechter and J. W. F. Valle, Phys. Rev. D 22, 2227 (1980). For recent reviews, see V. Barger, D. Marfatia, and K. Whisnant, Int, J,. Mod. Phys. E12, 569 (2003) [arXiv:hep-ph/0308123]; R. N. Mohapatra et al, arXiv:hep-ph/0510213; G. Altarelli, arXiv:hep-ph/0611117, and references therein.

[81] H. Ishimori, T. Kobayashi, H. Ohki, Y. Shimizu, H. Okada and M. Tanimoto, "NonAbelian Discrete Symmetries in Particle Physics," Prog. Theor. Phys. Suppl. 183, 1 (2010) doi:10.1143/PTPS.183.1 [arXiv:1003.3552 [hep-th]].

[82] W. Rodejohann, Status of the PMNS Matrix, in Proceedings, 10th Conference on Flavor Physics and CP Violation (Z. G. Zhao, J. X. Lu and Q. Wang), Hefei, China, 2012, [SLAC-eConf-C120521]. 
[83] E. Ma, "A(4) Symmetry and Neutrinos," Int. J. Mod. Phys. A 23, 3366 (2008) [arXiv:0710.3851 [hep-ph]]; E. Ma and G. Rajasekaran, "Softly broken A(4) symmetry for nearly degenerate neutrino masses," Phys. Rev. D 64, 113012 (2001) [hep-ph/0106291]. See also S. F. King and C. Luhn, "Neutrino Mass and Mixing with Discrete Symmetry," Rept. Prog. Phys. 76, 056201 (2013) [arXiv:1301.1340 [hep-ph]] for a review and references therein.

[84] N. Cabibbo, "Time Reversal Violation in Neutrino Oscillation," Phys. Lett. B 72, 333 (1978); L. Wolfenstein, "Oscillations Among Three Neutrino Types and CP Violation," Phys. Rev. D 18, 958 (1978).

[85] K. S. Babu, E. Ma and J. W. F. Valle, "Underlying A(4) symmetry for the neutrino mass matrix and the quark mixing matrix," Phys. Lett. B 552, 207 (2003) [hep-ph/0206292].

[86] C. Amsler et al. [Particle Data Group], Phys. Lett. B 667 (2008)

[87] H. Fritzsch and Z. z. Xing, Prog. Part. Nucl. Phys. 45 (2000)

[88] N. Cabibbo, Phys. Rev. Lett. 10, 531 (1963); M. Kobayashi and T. Maskawa, Prog. Theor. Phys. 49, 652 (1973).

[89] S. T. Petcov, "The Processes mu $\rightarrow$ e Gamma, mu $\rightarrow$ e e anti-e, Neutrino' $\rightarrow$ Neutrino gamma in the Weinberg-Salam Model with Neutrino Mixing," Sov. J. Nucl. Phys. 25, 340 (1977) [Yad. Fiz. 25, 641 (1977)] Erratum: [Sov. J. Nucl. Phys. 25, 698 (1977)] Erratum: [Yad. Fiz. 25, 1336 (1977)].

[90] E. Ma and A. Pramudita, "Exact Formula for $(\mu \rightarrow e \gamma)$ Type Processes in the Standard Model," Phys. Rev. D 24, 1410 (1981).

[91] P. Q. Hung, "Electroweak-scale mirror fermions, mu - i, e gamma and tau - i mu gamma," Phys. Lett. B 659 (2008) 585, [arXiv:0711.0733 [hep-ph]]. 
[92] P. Q. Hung and T. Le, "On neutrino and charged lepton masses and mixings: A view from the electroweak-scale right-handed neutrino model," JHEP 1509, 001 (2015) [arXiv:1501.02538 [hep-ph]].

[93] J. C. Pati and A. Salam, "Lepton Number as the Fourth Color," Phys. Rev. D 10, 275 (1974) [Phys. Rev. D 11, 703 (1975)]; R. N. Mohapatra and J. C. Pati, "A Natural Left-Right Symmetry," Phys. Rev. D 11, 2558 (1975); G. Senjanovic and R. N. Mohapatra, "Exact Left-Right Symmetry and Spontaneous Violation of Parity," Phys. Rev. D 12, 1502 (1975); G. Senjanovic, "Spontaneous Breakdown of Parity in a Class of Gauge Theories," Nucl. Phys. B 153, 334 (1979).

[94] K. A. Olive et al. (Particle Data Group), Chin. Phys. C, 38, 090001 (2014).

[95] J. Adam et al. [MEG Collaboration], "New constraint on the existence of the $\mu^{+} \rightarrow e^{+} \gamma$ decay," Phys. Rev. Lett. 110, 201801 (2013) [arXiv:1303.0754 [hep-ex]].

[96] F. Renga [MEG Collaboration], "Latest results of MEG and status of MEG-II," arXiv:1410.4705 [hep-ex].

[97] A. Hoecker and W. J. Marciano, http://pdg.lbl.gov/2013/reviews/rpp2013-rev-g-2-muonanom-mag-moment.pdf.

[98] A. M. Baldini et al. [MEG Collaboration], "Search for the Lepton Flavour Violating Decay $\mu^{+} \rightarrow e^{+} \gamma$ with the Full Dataset of the MEG Experiment," Eur. Phys. J. C 76, no. 8, 434 (2016) [arXiv:1605.05081 [hep-ex]].

[99] See the talk presented by K. Iwamoto (T2K Collaboration) at the ICHEP 2016, Chicago, August 2016.

[100] M. C. Gonzalez-Garcia, M. Maltoni and T. Schwetz, "Global Analyses of Neutrino Oscillation Experiments," Nucl. Phys. B 908, 199 (2016) [arXiv:1512.06856 [hep-ph]]. 
[101] P. Adamson et al. [NOvA Collaboration], "First measurement of electron neutrino appearance in NOvA," Phys. Rev. Lett. 116, no. 15, 151806 (2016) [arXiv:1601.05022 [hep-ex]].

[102] P. Adamson et al. [NOvA Collaboration], "First measurement of muon-neutrino disappearance in NOvA," Phys. Rev. D 93, no. 5, 051104 (2016) [arXiv:1601.05037 [hep-ex]].

[103] C. Dohmen et al. [SINDRUM II Collaboration], "Test of lepton-flavour conservation in $\mu-e$ conversion on titanium," Phys. Lett. B 317 (1993) 631.

[104] W. H. Bertl et al. [SINDRUM II Collaboration], "A Search for muon to electron conversion in muonic gold," Eur. Phys. J. C 47, 337 (2006).

[105] L. Bartoszek et al. [Mu2e Collaboration], "Mu2e Technical Design Report," arXiv:1501.05241 [physics.ins-det].

[106] Y. Kuno [COMET Collaboration], "A search for muon-to-electron conversion at J-PARC: The COMET experiment," PTEP 2013, $022 \mathrm{C} 01$ (2013).

[107] COMET Collaboration, Technical Design Report (2016).

[108] K. Knoepfel et al. [Mu2e Collaboration], "Feasibility Study for a Next-Generation Mu2e Experiment," arXiv:1307.1168 [physics.ins-det].

[109] R. J. Barlow, "The PRISM/PRIME project," Nucl. Phys. Proc. Suppl. 218, 44 (2011).

[110] M. Lindner, M. Platscher and F. S. Queiroz, "A Call for New Physics : The Muon Anomalous Magnetic Moment and Lepton Flavor Violation," arXiv:1610.06587 [hep-ph].

[111] P. Q. Hung, T. Le, V. Q. Tran and T. C. Yuan, "Lepton Flavor Violating Radiative Decays in EW-Scale $\nu_{R}$ Model: An Update," JHEP 1512, 169 (2015) [arXiv:1508.07016 [hep-ph]].

[112] Y. Kuno and Y. Okada, "Muon decay and physics beyond the standard model," Rev. Mod. Phys. 73, 151 (2001) [hep-ph/9909265]. 
[113] R. Kitano, M. Koike and Y. Okada, "Detailed calculation of lepton flavor violating muon electron conversion rate for various nuclei," Phys. Rev. D 66, 096002 (2002) Erratum: [Phys. Rev. D 76, 059902 (2007)] [hep-ph/0203110].

[114] J. C. Pati and A. Salam, "Lepton Number as the Fourth Color," Phys. Rev. D 10, 275 (1974) [Phys. Rev. D 11, 703 (1975)].

[115] R. N. Mohapatra and J. C. Pati, “A Natural Left-Right Symmetry,” Phys. Rev. D 11, 2558 $(1975)$.

[116] G. Senjanovic and R. N. Mohapatra, "Exact Left-Right Symmetry and Spontaneous Violation of Parity," Phys. Rev. D 12, 1502 (1975).

[117] G. Senjanovic, "Spontaneous Breakdown of Parity in a Class of Gauge Theories," Nucl. Phys. B 153, 334 (1979).

[118] H. B. Nielsen and M. Ninomiya, "No Go Theorem for Regularizing Chiral Fermions," Phys. Lett. 105B, 219 (1981).

[119] S. Chakdar, K. Ghosh, V. Hoang, P. Q. Hung and S. Nandi, "The search for electroweakscale right-handed neutrinos and mirror charged leptons through like-sign dilepton signals," to appear in Phys. Rev. D (2017) [arXiv:1606.08502 [hep-ph]].

[120] N. Cabibbo, "Time Reversal Violation in Neutrino Oscillation," Phys. Lett. B 72, 333 (1978).

[121] L. Wolfenstein, "Oscillations Among Three Neutrino Types and CP Violation," Phys. Rev. D 18, 958 (1978).

[122] C. F. Chang, P. Q. Hung, C. S. Nugroho, V. Q. Tran and T. C. Yuan, "Electron Electric Dipole Moment in Mirror Fermion Model with Electroweak Scale Non-sterile Right-handed Neutrinos," arXiv:1702.04516 [hep-ph].

[123] V. Cirigliano, R. Kitano, Y. Okada and P. Tuzon, "On the model discriminating power of $\mu-e$ conversion in nuclei," Phys. Rev. D 80, 013002 (2009) [arXiv:0904.0957 [hep-ph]]. 
[124] T. Suzuki, D. F. Measday and J. P. Roalsvig, Phys. Rev. C 35, 2212 (1987).

[125] M. A. Shifman, A. I. Vainshtein and V. I. Zakharov, "Remarks on Higgs Boson Interactions with Nucleons," Phys. Lett. 78B, 443 (1978).

[126] H. Fritzsch, "Calculating the Cabibbo Angle," Phys. Lett. 70B, 436 (1977). doi:10.1016/03702693(77)90408-7

[127] H. Georgi and C. Jarlskog, "A New Lepton - Quark Mass Relation in a Unified Theory," Phys. Lett. 86B, 297 (1979). doi:10.1016/0370-2693(79)90842-6

[128] P. Ramond, R. G. Roberts and G. G. Ross, "Stitching the Yukawa quilt," Nucl. Phys. B 406, 19 (1993) doi:10.1016/0550-3213(93)90159-M [hep-ph/9303320].

[129] P. Q. Hung and M. Seco, "Pure phase mass matrices from six-dimensions," Nucl. Phys. B 653, 123 (2003) doi:10.1016/S0550-3213(03)00007-5 [hep-ph/0111013].

[130] G. C. Branco and J. I. Silva-Marcos, "NonHermitian Yukawa couplings?,” Phys. Lett. B 331, 390 (1994). doi:10.1016/0370-2693(94)91069-3

[131] H. Fritzsch, "Weak Interaction Mixing in the Six - Quark Theory," Phys. Lett. 73B, 317 (1978). doi:10.1016/0370-2693(78)90524-5

[132] H. Nishiura, K. Matsuda and T. Fukuyama, "Lepton and quark mass matrices," Phys. Rev. D 60, 013006 (1999) doi:10.1103/PhysRevD.60.013006 [hep-ph/9902385].

[133] P.Q. Hung, T. Le, "Quark mass matrices from a model of neutrino masses and mixings", work in progress. 